\title{
Copyright
}

by

Steven Michael Horne

2013 
The Dissertation Committee for Steven Michael Horne Certifies that this is the approved version of the following dissertation:

\section{Multispectral Gamma-Ray Analysis Using Clover Detectors with Application to Uranium Fission Product Analysis}

\section{Committee:}

Sheldon Landsberger, Supervisor

Kevin Jackman, Co-Supervisor

Steven Biegalski

Erich Schneider

Malcolm Fowler 


\title{
Multispectral Gamma-Ray Analysis Using Clover Detectors with Application to Uranium Fission Product Analysis
}

$$
\text { by }
$$

Steven Michael Horne, B.S.; M.S.E.

\author{
Dissertation \\ Presented to the Faculty of the Graduate School of \\ The University of Texas at Austin \\ in Partial Fulfillment \\ of the Requirements \\ for the Degree of \\ Doctor of Philosophy
}

The University of Texas at Austin

May 2013 


\section{Dedication}

I would like to dedicate this work to my dear wife, Juliann, who has loved me unconditionally and supportively listened to my break-throughs, even when I didn't make sense. 


\section{Acknowledgements}

I would like to acknowledge my supervisor Dr. Sheldon Landsberger who introduced me to the field of Nuclear Engineering and has mentored me through this work as well as my Master's degree work. Dr. Kevin Jackman at Los Alamos National Laboratory provided essential oversight and guidance in many aspects of this research, and patiently taught me a number of necessary concepts to succeed with this work. Dr. Robert Rundberg of Los Alamos National Laboratory also gave great insight into this research and provided many of the initial algorithms and parameter settings that helped me start this project. I would also like to express my deep appreciation to the other members of my dissertation committee: Dr. Steven Biegalski and Dr.

Erich Schneider of The University of Texas at Austin, and Dr. Malcolm Fowler of Los Alamos National Laboratory.

This research was performed under the Nuclear Forensics Graduate Fellowship Program, which is sponsored by the US Department of Homeland Security, Domestic Nuclear Detection Office and the US Department of Defense, Defense Threat Reduction Agency. 


\title{
Multispectral Gamma-Ray Analysis Using Clover Detectors with Application to Uranium Fission Product Analysis
}

\author{
Steven Michael Horne, Ph.D. \\ The University of Texas at Austin, 2013
}

Supervisors: Sheldon Landsberger and Kevin Jackman

\begin{abstract}
A high-efficiency gamma-ray counting system has been built at Los Alamos National Laboratory for use in analyzing nuclear forensics samples. This system consists of two clover high-purity germanium detectors and is surrounded by a thallium-doped sodium iodide annulus. Special precautions have been taken to ensure the system has a low background. The system is connected to XIA Pixie-4 fast digitizers and collects data in list-mode. This work is split into two main parts. The first part describes the proper steps and techniques to initialize the settings of a detector system connected to fast digitizers in order to optimize the system for resolution and throughput. The various counting modes for this particular system are described in detail, including the benefits and drawbacks of each mode. Steps are then shown to characterize the system by obtaining efficiency curves for various counting modes and sample geometries. Because of the close counting geometry involved with this system, truecoincidence summing factors must be calculated, and are done so in part by measuring the peak-to-total ratios of the system in its various counting modes across a wide energy range. The dead-time for the system can be complicated due to the multiple inputs of the system. Techniques for calculating the dead-time of multiple-detector systems are discussed. The second part of this work shows the system's usefulness in analyzing nuclear forensics samples, specifically irradiated enriched uranium. Three fission product parent-daughter pairs of different lifetimes are analyzed over a course of six months. The activities of each nuclide are calculated at each time step. Age dating techniques using the parent-daughter pairs are discussed, as well as the detection limits of each nuclide for a range of sample ages. Finally,
\end{abstract}


avenues for further research are presented, as well as potential sources of error or uncertainty for this work. 


\section{Table of Contents}

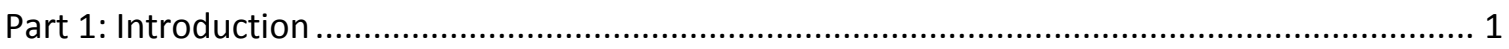

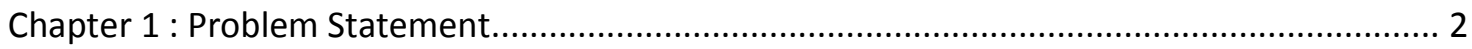

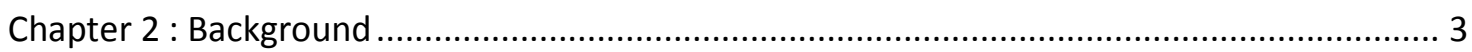

2.1 Traditional Gamma Spectroscopy with HPGe Detectors............................................ 3

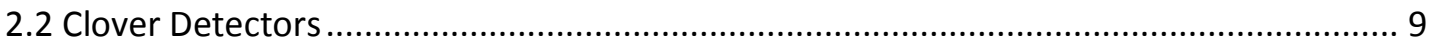

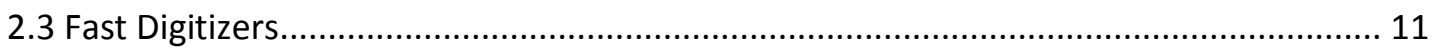

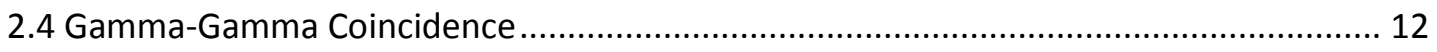

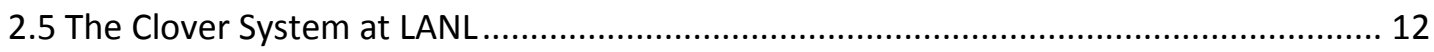

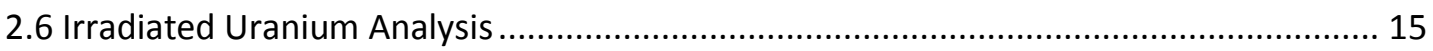

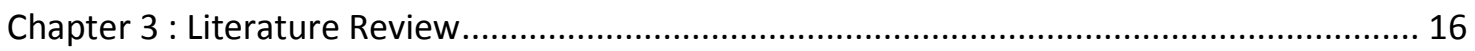

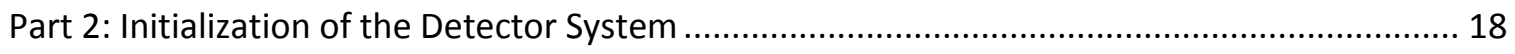

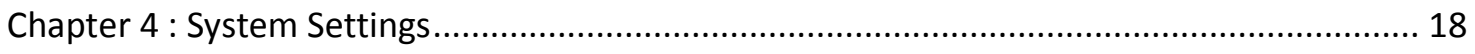

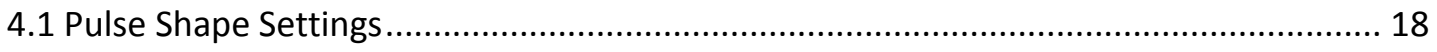

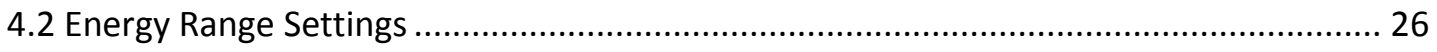

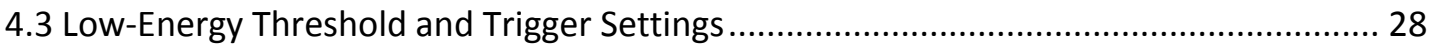

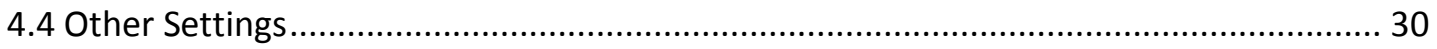

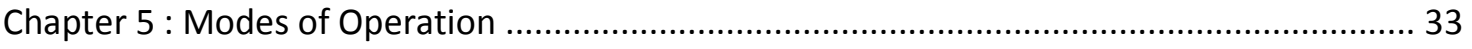

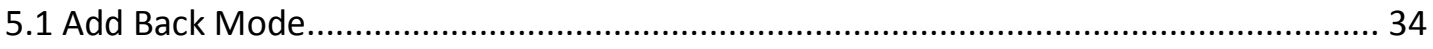

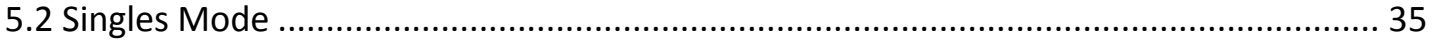

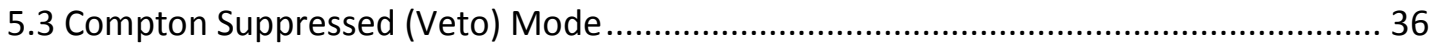

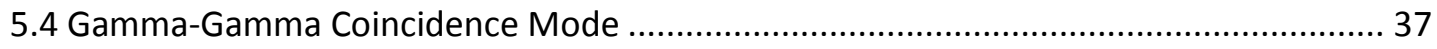

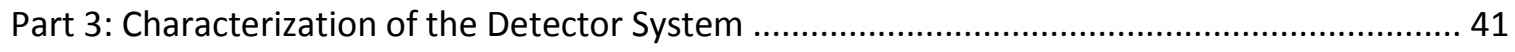

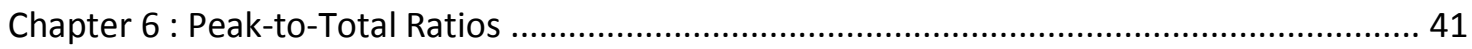




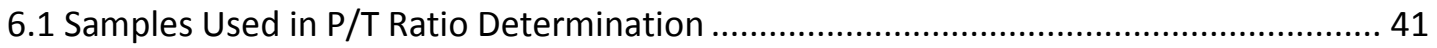

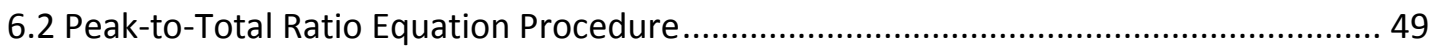

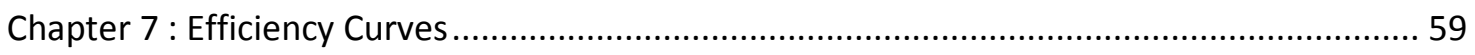

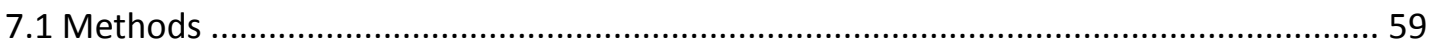

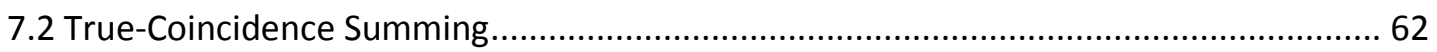

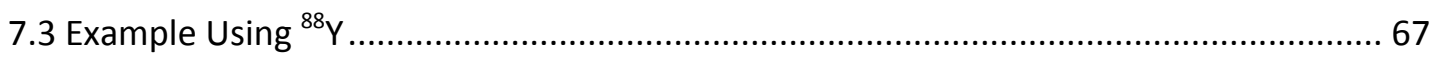

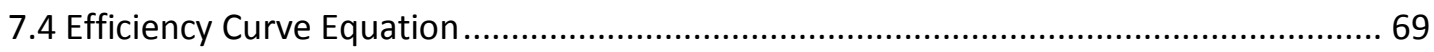

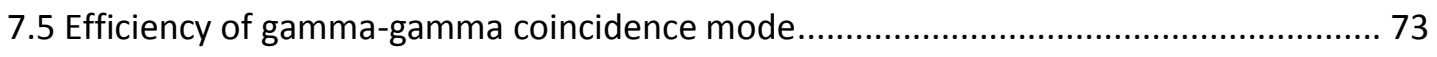

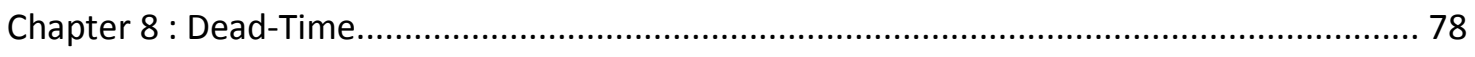

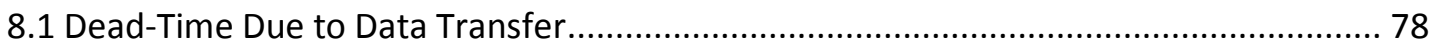

8.2 Dead-Time Due to Pulse Shaping and Trace Collection .............................................. 79

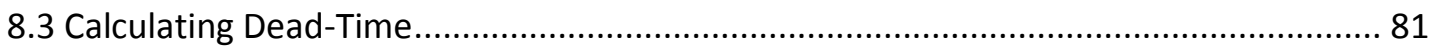

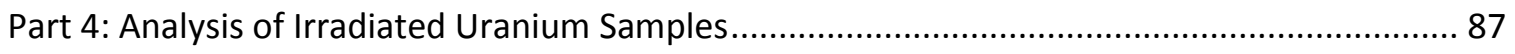

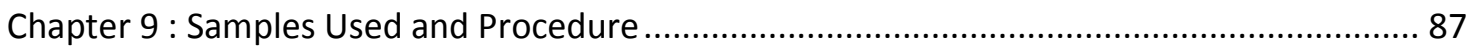

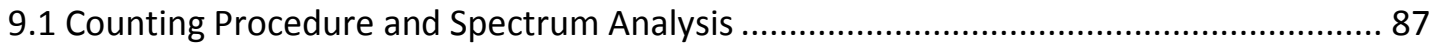

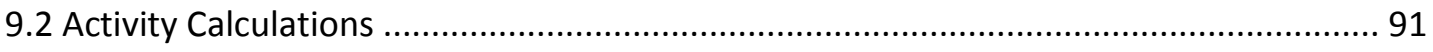

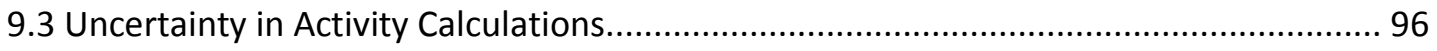

Chapter 10 : Analyzing Uranium Samples for Activity over Time......................................... 98

10.1 Activity Calculation Results from Sample 4126 ................................................... 102

10.2 Activity Calculation Results from Sample 4130 .................................................. 145

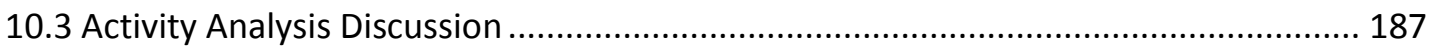

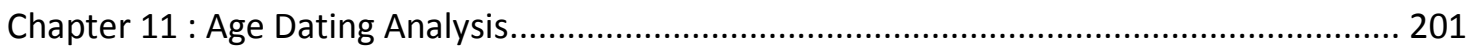

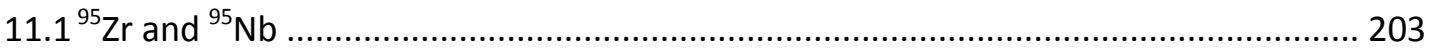

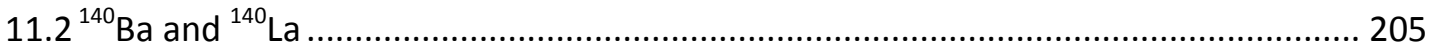




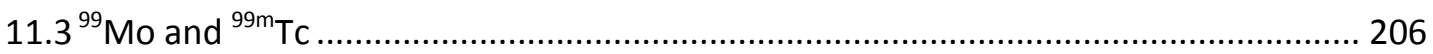

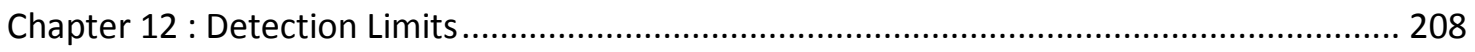

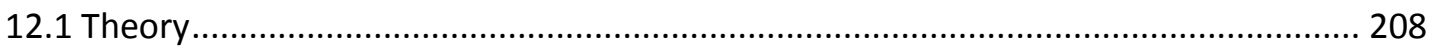

12.2 Detection Limits in Near Background Spectrum .................................................. 215

12.3 Detection Limits for Six Month Old Sample ........................................................ 217

12.4 Detection Limits for One Month Old Sample ........................................................ 219

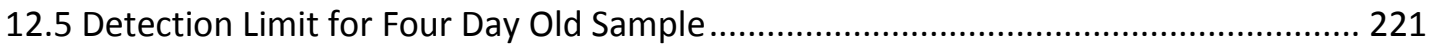

12.6 Comparison of Detection Limits with Traditional System ......................................... 222

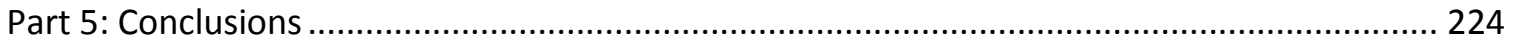

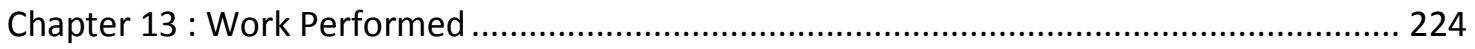

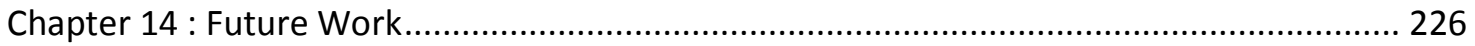

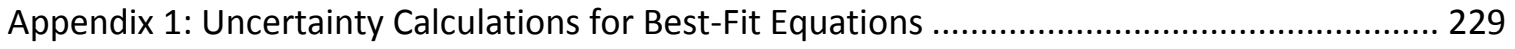

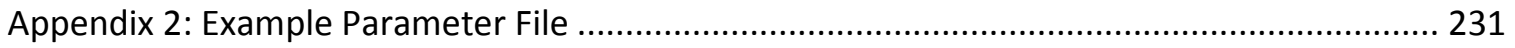

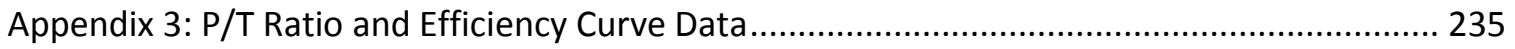

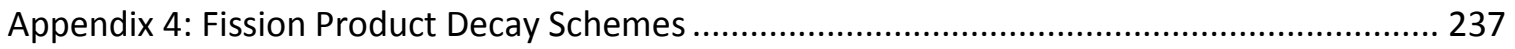

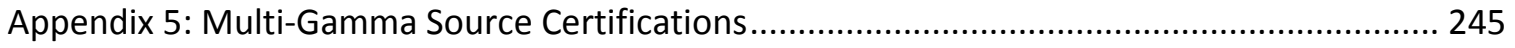

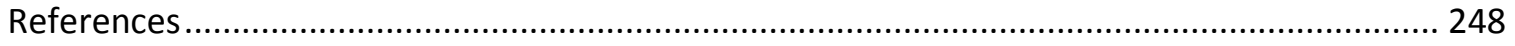




\section{List of Tables}

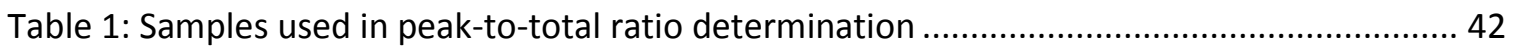

Table 2: Coefficients for P/T ratio equations for Add Back and Singles modes. ...........................57

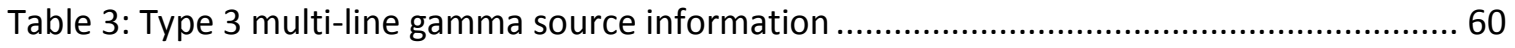

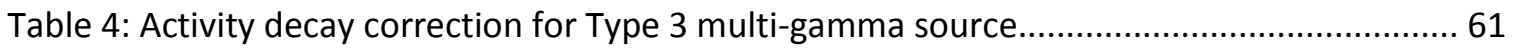

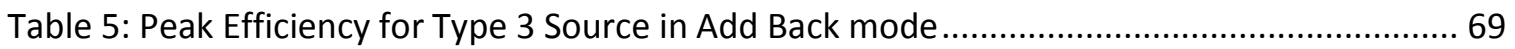

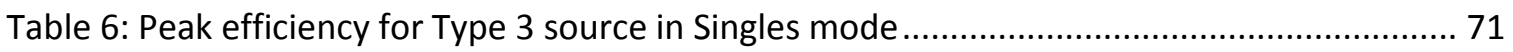

Table 7: Efficiency data points for Misty and Kerri Four-Crystal Add Back mode......................... 74

Table 8: Efficiency coefficients for Add Back Misty and Add Back Kerri modes. .......................... 86

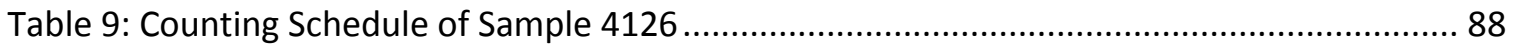

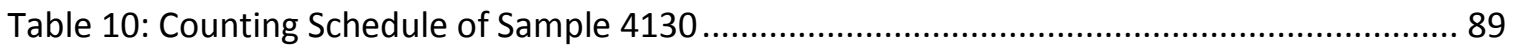

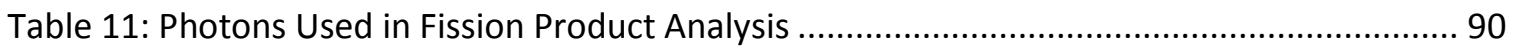

Table 12: Initial activity concentrations of parent nuclides as determined by separated

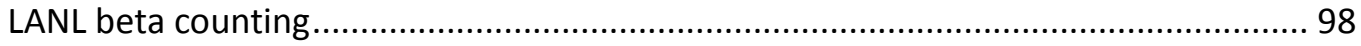

Table 13: Initial activity estimates of daughters from parent decay during irradiation ............. 100

Table 14: Thermal fission yields for parent and daughter nuclides ......................................... 100

Table 15: Initial activity estimates of daughters from direct fission yield ................................. 101

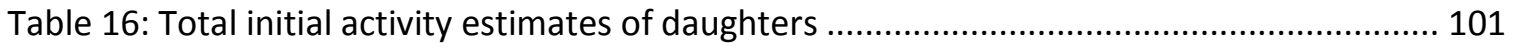

Table 17: Comparison of Add Back and Singles modes in detecting Zr-95/Nb-95 ..................... 189

Table 18: Comparison of Add Back and Singles modes in detecting Ba-140 ............................. 191

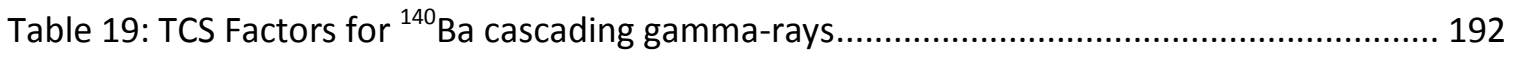

Table 20: Comparison of Add Back and Singles modes in detecting La-140 .............................. 193

Table 21: Effects of true coincidence summing in La-140 gamma-ray emissions....................... 193

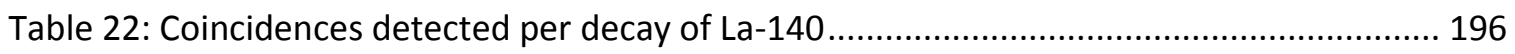

Table 23: Comparison of Add Back and Singles modes in detecting Mo-99/Tc-99m ................. 199

Table 24: Effects of true coincidence summing in Mo-99/Tc-99m gamma-ray emissions ......... 199

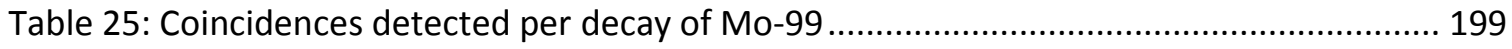

Table 26: Detection limits in Bq for a near background sample in Add Back mode. .................. 216

Table 27: Detection limits in Bq for a near background sample in Singles mode...................... 217 
Table 28: Detection limits in Bq for a 6-month old sample in Add Back mode.......................... 218

Table 29: Detection limits in Bq for a 6-month old sample in Singles mode. ............................ 219

Table 30: Detection limits in Bq for a 1-month old sample in Add Back mode.......................... 220

Table 31: Detection limits for a 1-month old sample in Singles mode. ................................... 220

Table 32: Detection limits for a 4-day old sample in Add Back mode........................................ 221

Table 33: Detection limits for a 4-day old sample in Singles mode. .......................................... 222

Table 34: Comparison of Detection Limits with Traditional System for 60 min Count Time ...... 223

Table 35: Coefficients for $\mathrm{P} / \mathrm{T}$ ratio equations of best fit for relevant counting modes. ............ 235

Table 36: Peak Efficiency for Type 3 (Disk) Source all counting modes .................................... 235

Table 37: Peak Efficiency for Type 1 (Point) Source all counting modes .................................... 236

Table 38: Coefficients for efficiency equations for Type 3 source ........................................... 236

Table 39: Coefficients for efficiency equations for Type 1 source ........................................... 236 


\section{List of Figures}

Figure 1: Cross sections of germanium and silicon for gamma interactions as a function

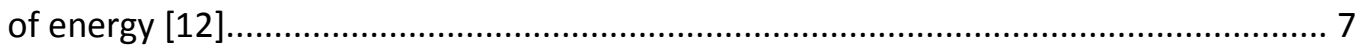

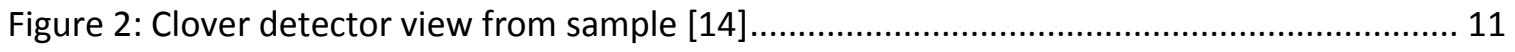

Figure 3: The clover system at LANL before the Nal(TI) shield had been installed ...................... 13

Figure 4: The clover system at LANL with the Nal(TI) shield installed ....................................... 14

Figure 5: Demonstration of shape filter and trigger algorithm for digitizer ............................... 20

Figure 6: Representation of trigger and shaping procedure for Pixie-4 digitizers ....................... 21

Figure 7: Dependence of peak resolution on the Energy Flat Top in Kerri ................................. 23

Figure 8: Dependence of peak resolution on the Energy Flat Top in Misty ................................. 23

Figure 9: The dependence of peak resolution on the Energy Rise Time in Kerri .......................... 24

Figure 10: The dependence of peak resolution on the Energy Rise Time in Misty ....................... 25

Figure 11: Possible deformed peak shape resulting from mismatched gain settings................... 27

Figure 12: Low energy noise from poor threshold and trigger settings...................................... 31

Figure 13: TAC spectrum for coincidences between the two clover detectors ............................ 34

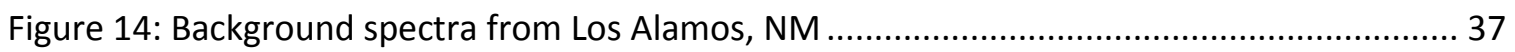

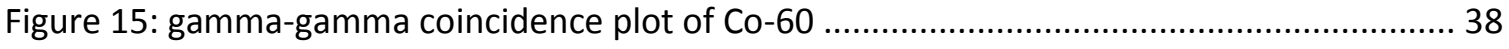

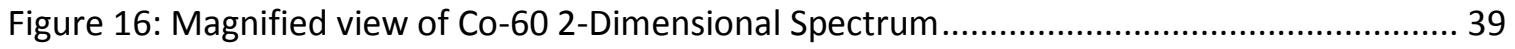

Figure 17: Co-60 gamma-gamma coincidence Spectrum Gated on $1173 \mathrm{keV}$............................ 40

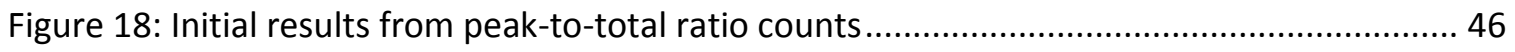

Figure 19: The difference in spectra in turning the $\mathrm{Cd}-109$ source upside-down......................... 47

Figure 20: The difference in spectra in turning the Sn-113 source upside-down........................ 47

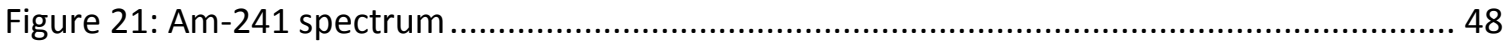

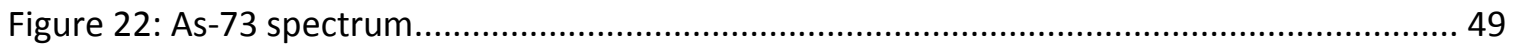

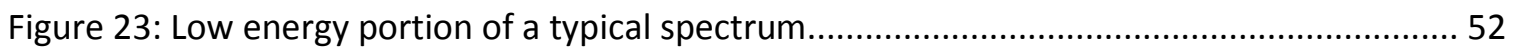

Figure 24: Some samples have X-rays in the low energy portion of their spectra. ...................... 52

Figure 25: Peak-to-total ratio equation for Singles and Add Back modes .................................. 58

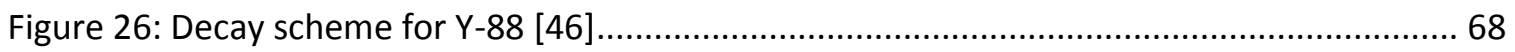

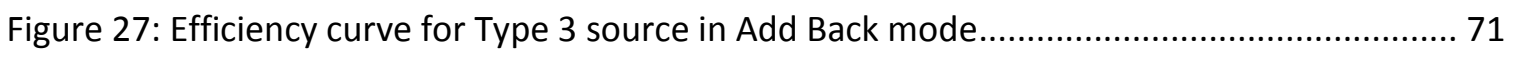




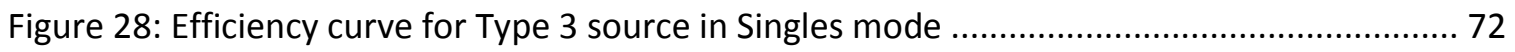

Figure 29: Efficiency curve for Type 3 source in Misty Add Back mode....................................... 74

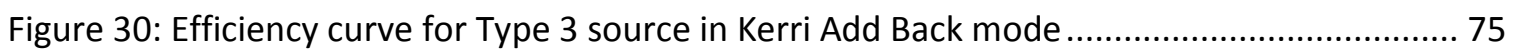

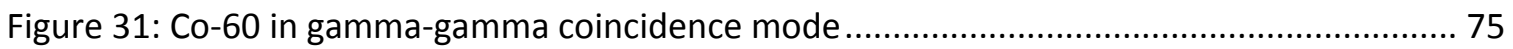

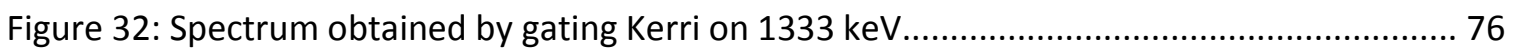

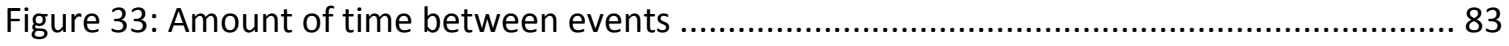

Figure 34: Average number of crystals photon deposits energy into ......................................... 85

Figure 35: Average number of modules photon deposits energy into ...................................... 86

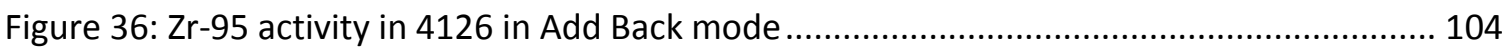

Figure 37: Zr-95 activity residuals in 4126 in Add Back mode.................................................... 105

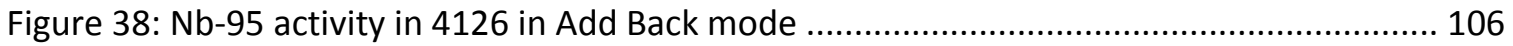

Figure 39: Nb-95 activity residuals in 4126 in Add Back mode ............................................... 107

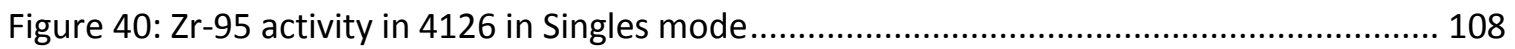

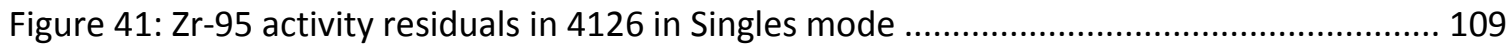

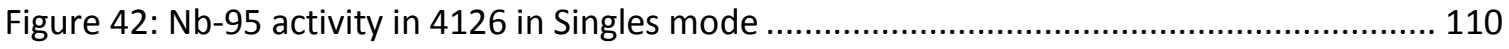

Figure 43: Nb-95 activity residuals in 4126 in Singles mode ................................................... 111

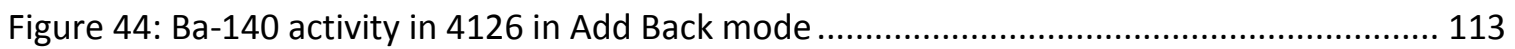

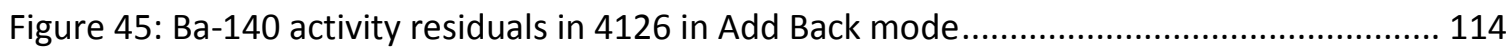

Figure 46: La-140 activity in 4126 in Add Back mode ............................................................. 115

Figure 47: La-140 activity residuals in 4126 in Add Back mode ............................................. 116

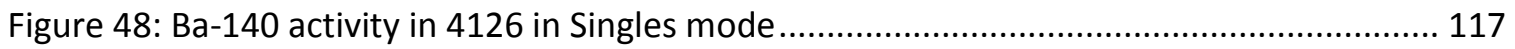

Figure 49: Ba-140 activity residuals in 4126 in Singles mode ................................................ 118

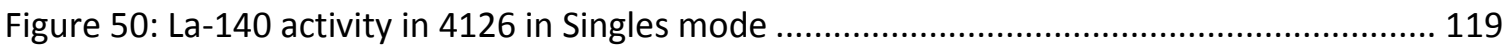

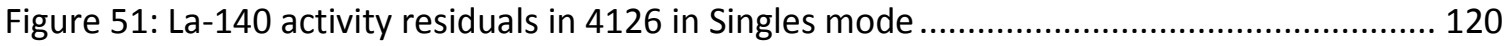

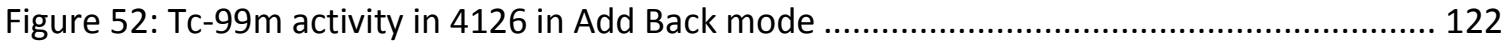

Figure 53: Tc-99m activity residuals in 4126 in Add Back mode ............................................. 123

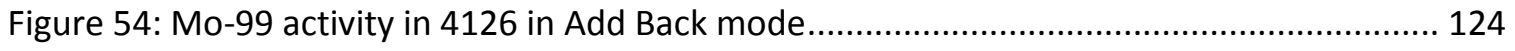

Figure 55: Mo-99 activity residuals in 4126 in Add Back mode .............................................. 125

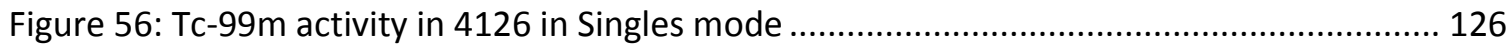

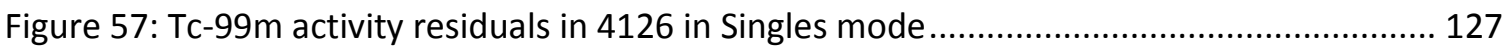




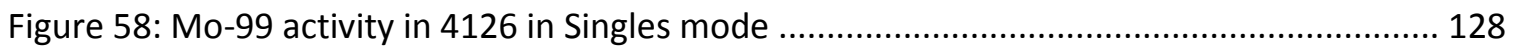

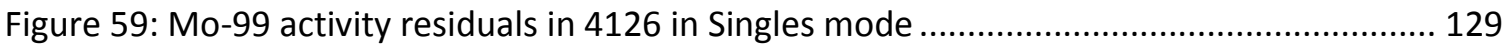

Figure 60: La-140 activity in 4126 in gamma-gamma coincidence mode gated on Kerri ........... 131

Figure 61: La-140 activity residuals in 4126 in gamma-gamma coincidence mode gated

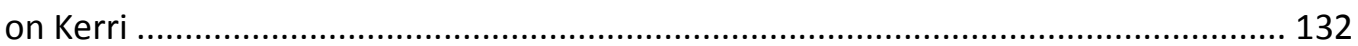

Figure 62: La-140 activity in 4126 in gamma-gamma coincidence mode gated on Misty........... 133

Figure 63: La-140 activity residuals in 4126 in gamma-gamma coincidence mode gated on Misty 134

Figure 64: Ba-140 activity in 4126 in gamma-gamma coincidence mode gated on Kerri. 136

Figure 65: Ba-140 activity residuals in 4126 in gamma-gamma coincidence mode gated on Kerri 137

Figure 66: Ba-140 activity in 4126 in gamma-gamma coincidence mode gated on Misty 138

Figure 67: Ba-140 activity residuals in 4126 in gamma-gamma coincidence mode gated on Misty

Figure 68: Mo-99 activity in 4126 in gamma-gamma coincidence mode gated on Kerri

Figure 69: Mo-99 activity residuals in 4126 in gamma-gamma coincidence mode gated on Kerri

Figure 70: Mo-99 activity in 4126 in gamma-gamma coincidence mode gated on Misty 143

Figure 71: Mo-99 activity residuals in 4126 in gamma-gamma coincidence mode gated on Misty 144

Figure 72: Zr-95 activity in 4130 in Add Back mode 146

Figure 73: Zr-95 activity residuals in 4130 in Add Back mode 147

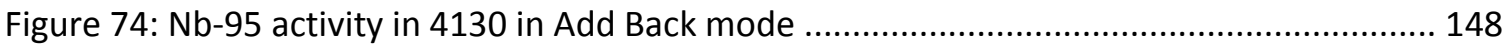

Figure 75: Nb-95 activity residuals in 4130 in Add Back mode ................................................ 149

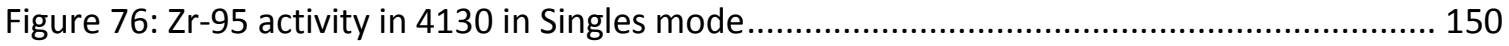

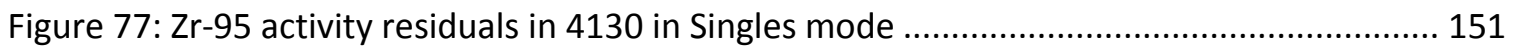

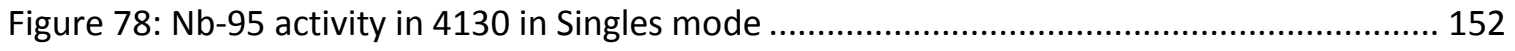

Figure 79: Nb-95 activity residuals in 4130 in Singles mode .................................................... 153

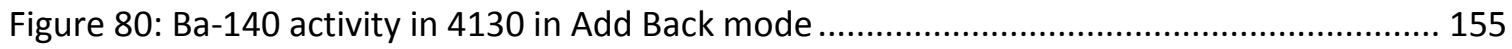

Figure 81: Ba-140 activity residuals in 4130 in Add Back mode.............................................. 156 


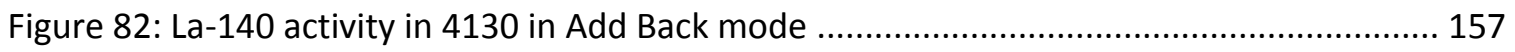

Figure 83: La-140 activity residuals in 4130 in Add Back mode .............................................. 158

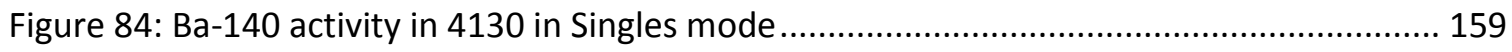

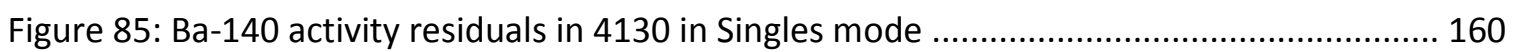

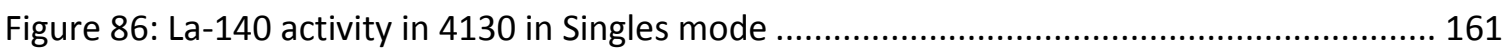

Figure 87: La-140 activity residuals in 4130 in Singles mode ................................................... 162

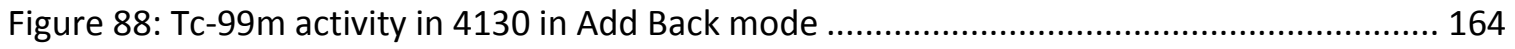

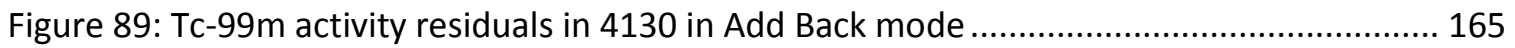

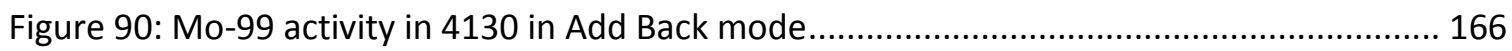

Figure 91: Mo-99 activity residuals in 4130 in Add Back mode ................................................ 167

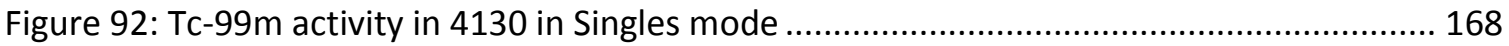

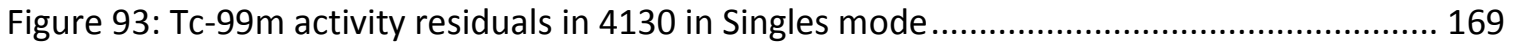

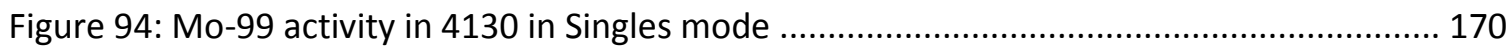

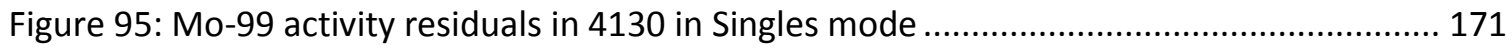

Figure 96: La-140 activity in 4130 in gamma-gamma coincidence mode gated on Kerri ........... 173

Figure 97: La-140 activity residuals in 4130 in gamma-gamma coincidence mode gated

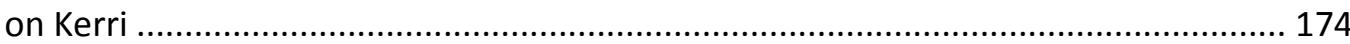

Figure 98: La-140 activity in 4130 in gamma-gamma coincidence mode gated on Misty.......... 175

Figure 99: La-140 activity residuals in 4130 in gamma-gamma coincidence mode gated

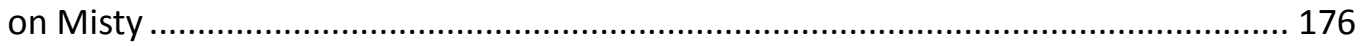

Figure 100: Ba-140 activity in 4130 in gamma-gamma coincidence mode gated on Kerri......... 178

Figure 101: Ba-140 activity residuals in 4130 in gamma-gamma coincidence mode gated on Kerri

Figure 102: Ba-140 activity in 4130 in gamma-gamma coincidence mode gated on Misty ....... 180

Figure 103: Ba-140 activity residuals in 4130 in gamma-gamma coincidence mode gated on Misty

Figure 104: Mo-99 activity in 4130 in gamma-gamma coincidence mode gated on Kerri 183

Figure 105: Mo-99 activity residuals in 4130 in gamma-gamma coincidence mode gated on Kerri 184

Figure 106: Mo-99 activity in 4130 in gamma-gamma coincidence mode gated on Misty 185 
Figure 107: Mo-99 activity residuals in 4130 in gamma-gamma coincidence mode gated

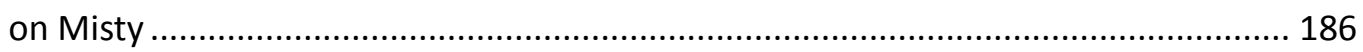

Figure 108: 4130 Add Back spectrum 4.5 days after zero time. ............................................ 188

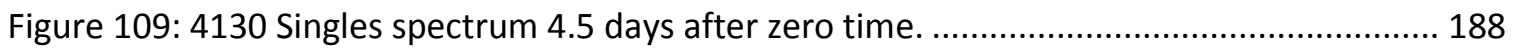

Figure 110: The $305 \mathrm{keV}$ peak is affected by an interfering peak around $306 \mathrm{keV}$..................... 194

Figure 111: Interference from $306 \mathrm{keV}$ peak removed when gating on $163 \mathrm{keV}$....................... 194

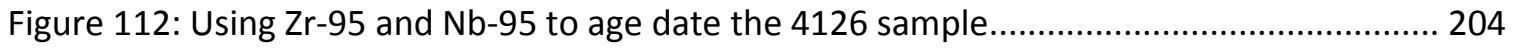

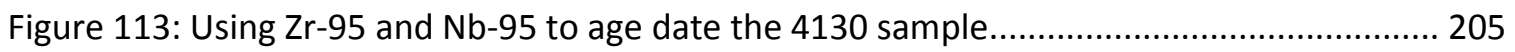

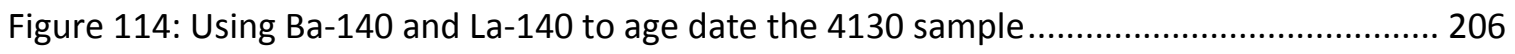

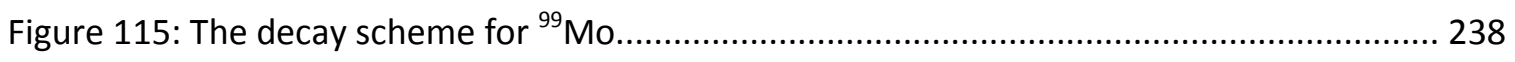

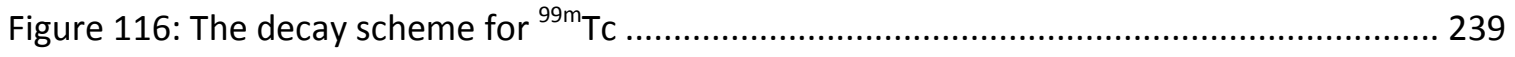

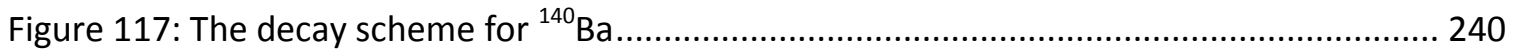

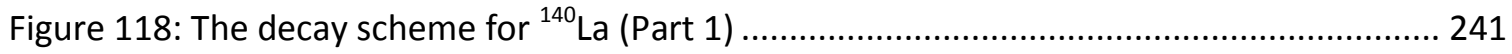

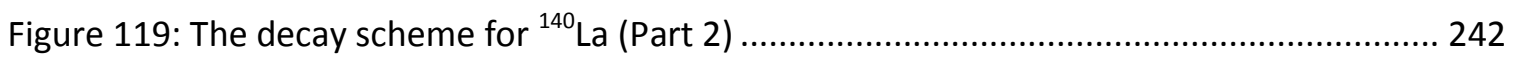

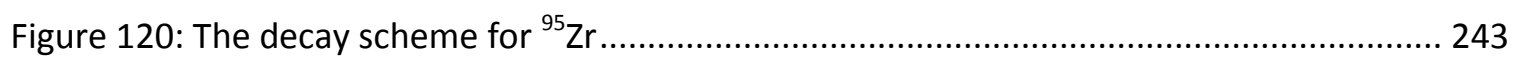

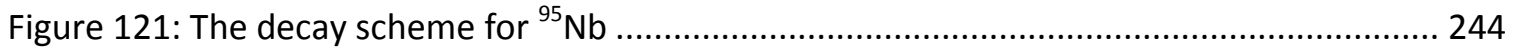

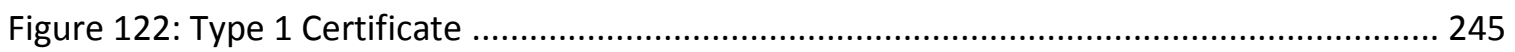

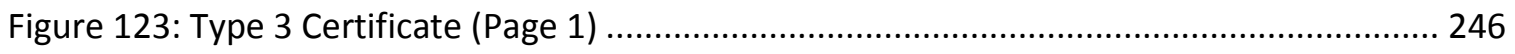

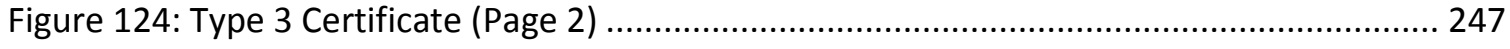




\section{Part 1: Introduction}

The analysis of nuclear materials, whether fresh or spent, using gamma spectroscopy is a commonly used technique which aims to gather pertinent information regarding the material's isotopic composition. Useful properties such as age, fission product activities, uranium enrichment information, and actinide activities may be determined by gamma spectroscopy. By understanding these properties, scientists and researchers can provide vital information to policy-makers and government officials (DOE, DoD, DHS, etc...) to aid in the nuclear forensics attribution process should a nuclear event occur.

Traditionally, gamma spectroscopy research is done by using analog systems with a single detector. More recently, Compton suppression systems involving a single solid-state detector and one or more high-efficiency scintillation detectors operated in anti-coincidence have been utilized. Important considerations in constructing these types of systems include cost, the speed at which results can be obtained, the size and portability of the system, and properties of the samples that the system should be expected to analyze. Should a nuclear forensics scenario arise, laboratory researchers would need to produce results as quickly as possible while still maintaining a high degree of confidence in the accuracy of the results. By using multiple detector systems connected to digitizers, results can be produced more quickly, and in many cases, the degree of confidence in the results can be increased through techniques that are not possible with traditional detector setups. One distinct advantage in using segmented detectors is the improvement in energy resolution over traditional detectors, which stems from the lower charge-collection time in the segmented detectors. 


\section{Chapter 1: Problem Statement}

This work has several aims which include the following:

1. to show the necessary steps involved in initializing and optimizing multipledetector systems with digitizers, which include spectral visualization, resolution optimization, low-energy threshold determination, timing correlation between detectors, and other parameter quantification;

2. to show the necessary steps involved in characterizing the performance of these systems such as obtaining and characterizing background spectra, generating efficiency curves for desired sample geometries and system counting modes, and peak-to-total ratios to correct for summing in the system in its various counting modes;

3. to show enhancements in detection limits and activity determination in isotopic analysis of nuclear samples (specifically samples containing uranium fission products) using these systems, which can then be compared with the performance of traditional systems.

One of the problems with current analysis techniques is sensitivity. When working with low-activity samples or when looking for radionuclides in the midst of strong interferences, analysis becomes quite difficult. One system being developed at Los Alamos National Laboratory (LANL) is composed of two high-purity germanium (HPGe) clover detectors, which are described in Section 2.2. The HPGe detectors are surrounded by a large thallium-doped sodium iodide ( $\mathrm{Nal}(\mathrm{TI}))$ scintillation annulus. This system has been created to be used for lowbackground analysis when high sensitivity is needed. By analyzing gamma-emitting samples using normal, Compton-suppressed, and gamma-gamma coincidence modes, important information about the material can be more precisely and accurately defined. This work will use the system at LANL to analyze three uranium fission product parent-daughter pairs: ${ }^{99} \mathrm{Mo} /{ }^{99 \mathrm{~m}} \mathrm{Tc}$, ${ }^{140} \mathrm{Ba} /{ }^{140} \mathrm{La}$, and ${ }^{95} \mathrm{Zr} /{ }^{95} \mathrm{Nb}$.

Lastly, it should be mentioned that the setup of a multiple detector system can quickly become quite complex. There are many parameters which must be set correctly and can have profound influences on the output spectra. This work will explain the steps necessary to take a given detector setup and characterize it such that good analysis can be performed. 


\section{Chapter 2: Background}

Gamma spectroscopy has been used extensively for over 60 years in analyzing radioactive materials $[1,2]$. It was initially done using scintillation materials, however as semiconductor detectors were developed in the 1960's [3] they became more popular because of their much improved energy resolution when compared to scintillation counters [4]. In semiconductor detectors, photons interact with the electrons in the detector, freeing them from their covalent bonds in the semiconductor material. These energetic electrons collide with and free other electrons in the semiconductor, which are then collected and analyzed. The total charge of the released electrons is proportional to the energy absorbed by the detector.

\subsection{Traditional Gamma Spectroscopy with HPGe Detectors}

In general, semiconductor detectors are composed of crystalline materials with a periodic lattice structure [4]. These lattices create energy bands for electrons that exist in the detector. The two bands that generally exist in semiconductors are the valence band and the conduction band. Electrons residing in the valence band are covalently bonded to specific lattice sites and cannot move freely throughout the material [4]. Electrons residing in the conduction band are not bound to any specific lattice site and are free to migrate throughout the material. The two energy bands are separated by the bandgap, which is sometimes called the "forbidden zone." In a pure material, no electrons can reside in the forbidden zone. For an electron to transfer from the valence band to the conduction band, the electron must accrue enough energy to cross the bandgap. This energy can be obtained either by thermal excitation or an outside source such as a photon.

The nature of the semiconductor material can be changed by doping the material with impurities [4]. The impurity can be a donor or an acceptor. A donor impurity is one that has a single electron in its outermost shell. The donor will easily give up this electron in order to have its electron shells completely filled. An acceptor impurity is an element that is one short of having a filled outermost shell. The acceptor will readily receive an electron in order to fill this shell. By doping a semiconductor, the material will become either p-type or n-type. A p-type material is one that has been doped with an acceptor impurity while an n-type material has been doped with a donor impurity. By doping one side of a p-type semiconductor with a donor impurity (or doping one side of an n-type detector with an acceptor impurity) a p-n junction can 
be established in the semiconductor. This $p$-n junction will cause an electric field to be established within the material due to electrons from the donor impurities drifting to the acceptors and holes from the acceptors drifting to the donors. The region in which nearly all of the charge carriers have been swept out or are intrinsically absent is termed the "depletion region." By applying a reverse bias voltage to this material (attaching a positive voltage on the positive side of the detector with respect to the negative side), the potential across the detector can be increased such that electrons in the conduction band will have a high drift velocity and the collection time will be short [4]. In this scenario, the positively biased side will already have a high concentration of holes and the negatively biased side will have a high concentration of electrons. Therefore, the reverse current across the $p-n$ junction is relatively low. The $p-n$ junction serves to allow relatively free flow of current in one direction and a large resistance to current in the other direction [4]. If the reverse bias voltage is increased enough, the depletion region will extend across the entire detector active volume. In practice, the $p-n$ junction is formed by creating electrical contacts on each side of the detector that are doped to be either p-type or n-type material. These are termed $n^{+}$and $p^{+}$contacts. The depletion region extends from barrier between the crystal and the oppositely doped electrical contact [4].

Coaxial detectors are produced in a cylindrical shape with a cylindrical hole carved out in the center of the crystal. The outside surface of the detector serves as one side of the electrical contact while the inside surface of the hole serves as the opposite electrical contact. The $n^{+}$and $p^{+}$contacts themselves are considered "dead layers" because photons that interact in these regions do not contribute to the charge collection process. The thickness of the dead layer is different for $n^{+}$and $p^{+}$contacts. For $n^{+}$contacts, the dead layer is a few tenths of a micrometer; whereas for $p^{+}$contacts, the dead layer is several hundred micrometers [4]. For photons above about $200 \mathrm{keV}$, the dead layer thicknesses do not matter, but the dead layer can affect the efficiency of the active volume for photons below this energy.

Gamma spectroscopy using HPGe detectors is performed using a high-purity germanium crystal inside a cryostat that is cooled to $77 \mathrm{~K}$ (generally with liquid nitrogen, though the temperature can be maintained in other ways [5]). The cooling of the crystal to this temperature widens the bandgap slightly and is cool enough such that thermally induced excitations across the bandgap are minimized [4]. At any temperature above absolute zero, 
thermal vibrations have a probability of producing enough energy for the electrons to jump the gap between the valence band and conduction band; however, at liquid nitrogen temperatures, the probability that thermal vibrations will give electrons enough energy to leave the valence band is very low [4]. The germanium crystal is polarized with a high voltage power source, creating a reverse bias across the active region of the detector. This creates an electric field across the detector which guides the electron-hole pairs formed from interactions with ionizing radiation. The average energy expended per electron-hole pair produced in the detector for germanium at $77 \mathrm{~K}$ is about $2.96 \mathrm{eV}$ [4]. Because so little energy is expended per electron-hole pair compared with the energy of the incoming photons, many secondary and tertiary interactions and scattering events occur within the germanium crystal. Gamma-rays emitted from radioactive materials are generally in the $\mathrm{keV}$ to $\mathrm{MeV}$ range, so the number of electronhole pairs created is in the thousands to millions for a given photon. These electrons and holes are attracted by the high voltage polarization and are ultimately collected by a capacitor in the preamplifier.

A resistive feedback amplifier is used for measuring the charge collected on the input capacitor. A simplified model consists of a capacitor connected in parallel with a resistor [4]. As charge is collected on the capacitor, it slowly leaks away through the resistor. The time constant associated with this circuit is quite large compared with the collection time of the charge so that the majority of the freed charge is collected on the capacitor before it dissipates through the resistor. The charge on this capacitor is continuously measured, and these measurements produce a pulse that is transported through amplifying and shaping electronics. The end result is read and binned according to the pulse height, which is proportional to the energy deposited in the detector. By reading many of these events, a spectrum can be produced. The mechanism of interaction between the photons and the electrons in the semiconductor generally fall into three types: the photoelectric effect, Compton scattering, and pair production [4].

The photoelectric effect occurs when a photon is completely absorbed by an electron [6]. The energy of the photon is completely transferred to this electron, giving the electron a kinetic energy of [4]: 


$$
E_{e^{-}}=h v-E_{b}
$$

where:

$E_{e^{-}}$is the energy of the photoelectron

$h v$ is the original energy of the photon

$E_{b}$ is the binding energy of the electron in its original shell

The binding energy for electrons in germanium is $11.1 \mathrm{keV}$ for the $K$ shell and between 1.2 and $1.4 \mathrm{keV}$ for the $L$ shell [7]. In addition to a photoelectron, the interaction also creates an ionized atom that has a vacancy in one of its shells [4]. The hole in this shell is quickly filled with another electron and often does so by emitting one or more characteristic X-rays. The X-rays are generally reabsorbed close to the emission site, but if the interaction occurs close to the surface of the detector, the X-ray can escape. Emission of Auger electrons can also occur in place of X-ray emission. Auger electrons are produced when a photon originating from the nucleus is absorbed by an electron in the same atom. This electron is ejected from the atom with an energy equal to that of the photon minus the binding energy of the electron. The excited photoelectron then collides with and frees other electrons. As these freed electrons in the conduction band excite bound electrons in the valence band, they lose some of their kinetic energy. Eventually, the electrons in the conduction band stop interacting with the electrons in the valence band and their motion is influenced heavily by the electric field, which is produced by the reverse biased semiconductor. The total charge of these freed electrons is proportional to the original energy of the absorbed photon [4]. By collecting this charge on the resistive feedback amplifier, the total freed charge can be measured and the energy transferred in the original interaction can be approximated. As seen in Figure 1, the cross section for the photoelectric effect compared with other interactions is dominant at energies below about 200 keV. Above this threshold, the photoelectric effect is still possible, but it is not the dominant interaction. Generally, the photoelectric effect is most probabilistic for electrons in the $K$ shell, which is the most tightly bound shell in the atom. Exceptions to this statement include photons below the binding energy of the $K$ shell which are more likely to interact with electrons in less tightly bound shells [4]. 


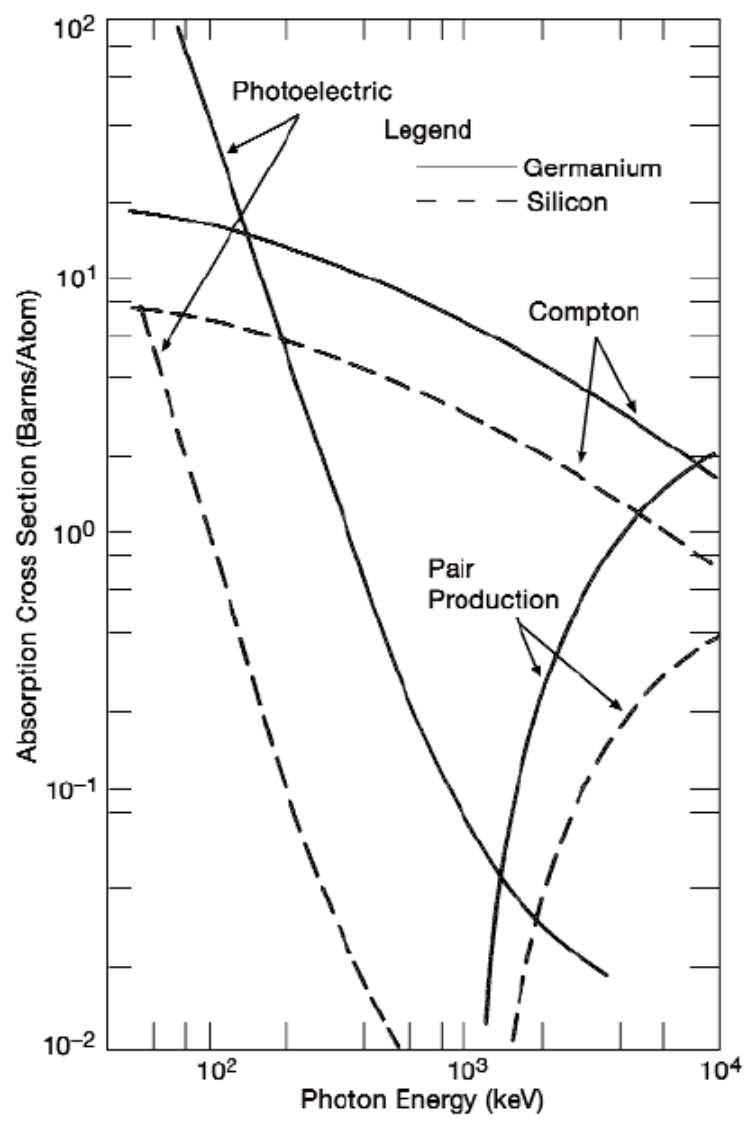

Figure 1: Cross sections of germanium and silicon for gamma interactions as a function of energy [12]

Compton scattering occurs when a gamma-ray interacts elastically with an electron such that only part of the energy of the photon is transferred to the electron [8]. This can be visualized by imagining a cue ball striking another billiard ball initially at rest so that both balls have kinetic energy and travel in different directions. The electron is not truly at rest, as it has some bound momentum from its interaction with the nucleus of the atom; however, to a first approximation, it can be considered at rest. The amount of energy transferred to the electron is related to the scattering angle of the interaction by [9]: 


$$
h v^{\prime}=\frac{h v}{1+\frac{h v}{m_{0} c^{2}}(1-\cos \theta)}
$$

where:

$h v^{\prime}$ is the energy of the scattered photon

$h v$ is the energy of the original photon

$m_{0} c^{2}$ is the rest mass energy of the electron $(0.511 \mathrm{MeV})$

$\theta$ is the scattering angle of the photon

The energy imparted to the electron is given by:

$$
E_{e^{-}}=h v-h v^{\prime}-E_{b}
$$

The electron with energy given in Equation (2.3) then undergoes the same processes described above for the photoelectric effect, and this energy is then recorded in the spectrum. As can be observed in Equation (2.2), there is an upper bound on the energy that can be imparted to the electron. This upper bound occurs when $\theta=\pi$, which corresponds to a complete reversal in photon direction. Because the angle of scattering and the energy of the incident photon are generally unknown, Compton scattering events generally contribute to the low-energy continuum of the spectrum and are undesirable. Oftentimes with higher energy photons, multiple Compton scatter events can occur in the same detector before a photoelectric interaction occurs [4]. Because photons travel approximately $30 \mathrm{~cm}$ in one $\mathrm{ns}$, this typically all happens within the timing resolution of the detector and therefore the sum of all energies transferred within the crystal is recorded in the spectrum. Even though the energy is transferred within the timing resolution of the detector, the interactions will take place at different positions in the detector. By observing the shapes of the pulses produced by the detector, one can determine some information about locations of interactions and perhaps even the number of Compton scatters that occurred in the detector [10]. Figure 1 shows that the Compton scattering cross section for germanium is dominant for photons with energy between about $200 \mathrm{keV}$ up to about 9.0 MeV.

Pair production occurs when a high energy photon interacts directly with the Coulomb field of the nucleus of an atom. If the photon has enough energy, the photon can spontaneously 
transform into an electron-positron pair [11]. This pair has a combined rest-mass energy of $1.022 \mathrm{MeV}$, and therefore, this interaction does not occur for photons below this cutoff energy. The combined kinetic energy of the newly-produced particles is given by [4]:

$$
E_{e^{-}}+E_{e^{+}}=h v-2 m_{0} c^{2}
$$

where $E_{e}+$ is the kinetic energy of the positron. The electron then interacts within the detector as any other electron in the conduction band would, freeing other electrons and adding charge to the capacitor [4]. The positron also interacts with the semiconductor material until it loses enough energy to annihilate with an electron. This positron-electron pair generally annihilates into two photons traveling in opposite directions and having $511 \mathrm{keV}$ each. These photons can then either escape from the detector without further interaction, undergo Compton scattering within the detector and escape, or be fully absorbed in the detector. All of these interactions associated with pair production generally take place within the resolving time of the detector. A single escape peak is observed when a pair production interaction takes place and one of the $511 \mathrm{keV}$ annihilation photons escapes from the detector, but the remainder of the energy from the photon is absorbed in the detector [4]. The resultant spectrum input is seen as the full energy of the photon minus $511 \mathrm{keV}$. A double escape peak is observed when both $511 \mathrm{keV}$ annihilation photons escape from the detector, leaving the full energy deposited in the detector minus $1022 \mathrm{keV}$ [4]. Pair production is the dominant interaction for gamma-rays in the high energy region, above about $9 \mathrm{MeV}$ as seen in Figure 1.

Though the field of gamma spectroscopy is several decades old, new advancements and research initiatives are still being performed to enhance current capabilities. Among these advancements include the development of segmented detectors, fast digitizers, pulse-shape discrimination, and modern techniques of enhancing spectra based on timing characteristics of specific events. In this work, all of these advancements will be applied to analyzing irradiated uranium samples to further characterize fission products of uranium samples and age-dating of these samples.

\subsection{Clover Detectors}

As semiconductor detectors, and specifically HPGe detectors, have been further developed, one of the main goals has been to make these detectors as efficient and precise as possible. By growing the germanium crystals in different shapes and sizes, researchers have 
been able to create detectors with higher and higher efficiencies with specific optimizations for various energy ranges. With increasing mass, the crystals lose some of their ability to transport electrons across the whole crystal to the cathode [12]. This gives rise to decreased charge collection, increased charge trapping, higher dead-times, and degraded peak resolution in the spectrum [12]. To overcome this problem, researchers have developed the segmented detector. This detector consists of several HPGe crystals that are grouped closely together in a single cryostat. By connecting these crystals together with proper timing modules or to digitizers, the crystals can work together to effectively simulate one large crystal. This is done by using "Add Back" mode, in which energies of photons that are observed in neighboring crystals within the timing window are added together. This technique assumes that these neighboring crystal events are Compton scatter events that would have been lost to the Compton continuum otherwise.

By increasing the active volume of the detector, the Compton continuum is reduced and the full-energy peak areas are increased. However, if the solid angle of the detector compared with the sample is large, true-coincidence summing can occur. True-coincidence summing takes place when two or more photons emitted from the same decaying nucleus interact in the detector. This occurs with nuclides that have a complex decay scheme and emit more than one photon per decay. If the geometric attenuation is reduced by increasing the solid angle of the detector system, energy depositions from true-coincidence events are more likely to be summed together. This contributes to sum peaks and the spectral continuum [13]. Clover HPGe detectors are a type of segmented detector in which the four germanium crystals are shaped like a clover-leaf as in Figure 2. 


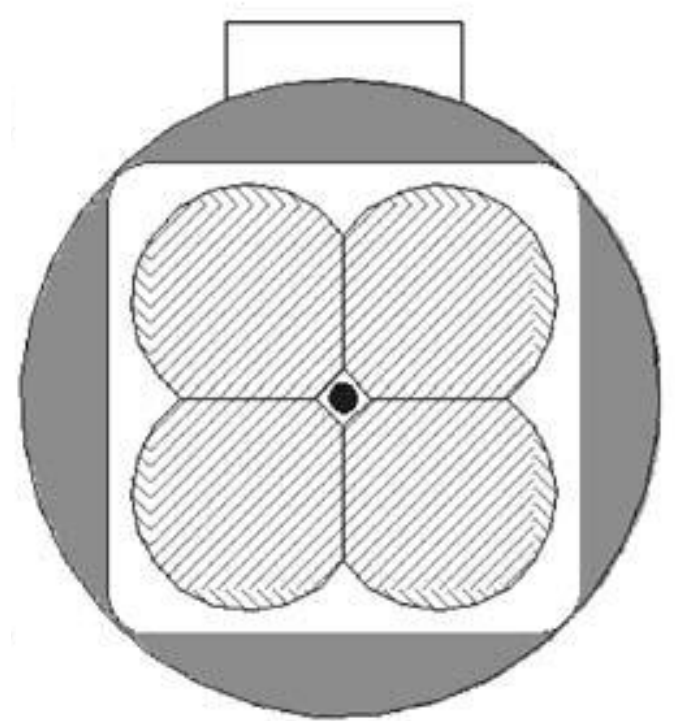

Figure 2: Clover detector view from sample [14]

\subsection{Fast Digitizers}

Traditional systems generally use analog electronics modules in order to shape the detector pulses resulting from photon interactions. This is done to capture the full energy deposition by the photon in the detector accurately and precisely by calculating the amplitude of the charge collection in the preamplifier [4]. Recent advancements in sampling rates and bit resolutions have allowed scientists to connect their detectors directly to fast-digitizer modules. These modules convert the preamplifier pulses from the detector directly to digital data, which can be analyzed and shaped internally. By doing this, the need for copious amounts of electronic modules is eradicated, making for potentially cheaper and smaller systems particularly when multiple detectors are involved. The pulse processing time may also be reduced compared to analog systems, allowing for the potential of less dead-time [15]. The use of digital filters, such as trapezoidal filters, also helps smooth out variations in the pulse amplitude resulting from charge trapping and incomplete charge collection [4]. The digitizer modules have the ability to record the photon energy of an event and attach a timestamp to the event; the method of taking data in this fashion is termed "list-mode." By taking data in listmode, one is able to take data from a single count and construct multiple spectra from it, examples of which include Add Back, Compton-suppressed, and gamma-gamma coincidence spectra. One also has the capability of recording waveforms to study the shape of the incoming 
pulses and discriminating the origin of the pulse based on various pulse-shape analysis techniques [10].

\subsection{Gamma-Gamma Coincidence}

Gamma spectroscopy identifies radionuclides by the gamma lines associated with that nuclide. One of the barriers to using these traditional methods of gamma spectroscopy is spectral interference. Spectral interferences can occur when a peak of interest overlaps with a peak from another nuclide in the sample or when peaks are difficult to discern from the underlying continuum. Gamma-gamma coincidence aims to use multiple detectors to analyze nuclides that have cascading gamma lines. This technique creates a two-dimensional spectrum by recording an event only if a photon is detected in each detector within a specified timing window. The two-dimensional spectrum is populated by adding a count to the two-dimensional bin corresponding to the two energies seen by the detectors. The spectrum can then be gated on one detector within a small energy range and analyzed by traditional one-dimensional methods. By using this technique, some interference events can be completely separated and the continuum below the peak can be decreased significantly.

\subsection{The Clover System at LANL}

The system at LANL has been created specifically for analyzing low-level activity samples and reducing spectral interferences via gamma-gamma coincidence mode. The use of fastdigitizers was chosen in place of analog systems for use with list-mode data acquisition. Figure 3 and Figure 4 show the system before and after the Nal(TI) shield was installed. The system is equipped with two HPGe clover detectors. Each clover detector has four n-type coaxial germanium crystals with $\sim 20 \%$ relative efficiency for each crystal. Oftentimes, a detector is described in terms of its relative efficiency as opposed to its absolute efficiency. The relative efficiency is defined as the absolute efficiency of the detector measuring $1333 \mathrm{keV}$ photons at a distance of $25 \mathrm{~cm}$ divided by the absolute efficiency of a 3" $\times 3$ " Nal(TI) detector in these conditions. These detectors are held by a stand made with thermoplastic materials (Delrin, $\mathrm{PEEK}, \mathrm{PEI}$, and $\mathrm{ABS}$ ) in order to reduce contamination present in most structural materials. The detectors face each other in a $180^{\circ}$ geometry with a separation of about $1 / 8^{\prime \prime}$. This distance can be adjusted with the use of a hand-crank in order to accommodate samples that are taller than 1/8". The HPGe clover detectors are surrounded by a $\sim 2$ " thick Nal(TI) annulus used for passive 
shielding and active Compton suppression. The Nal(TI) annulus is encased in oxygen-free high conductivity copper, which has lower intrinsic radioactivity than traditionally used aluminum. Many of the internal components in the detector system were constructed from lowbackground aluminum and copper as well. The Nal(TI) annulus is equipped with eight photomultiplier tubes (PMTs), with two PMTs for each quadrant of the annulus. The sections are separated to isolate light pulses in each quadrant. The room containing the system is clad with $\sim 8$ " of pre-WWII steel which essentially eliminates the need for a lead shield around the detector and is free from contamination due to nuclear fallout. The room is equipped with a High Efficiency Particulate Air (HEPA) filtration system, which continuously circulates the air in the room and helps filter out any radon gas in the room.

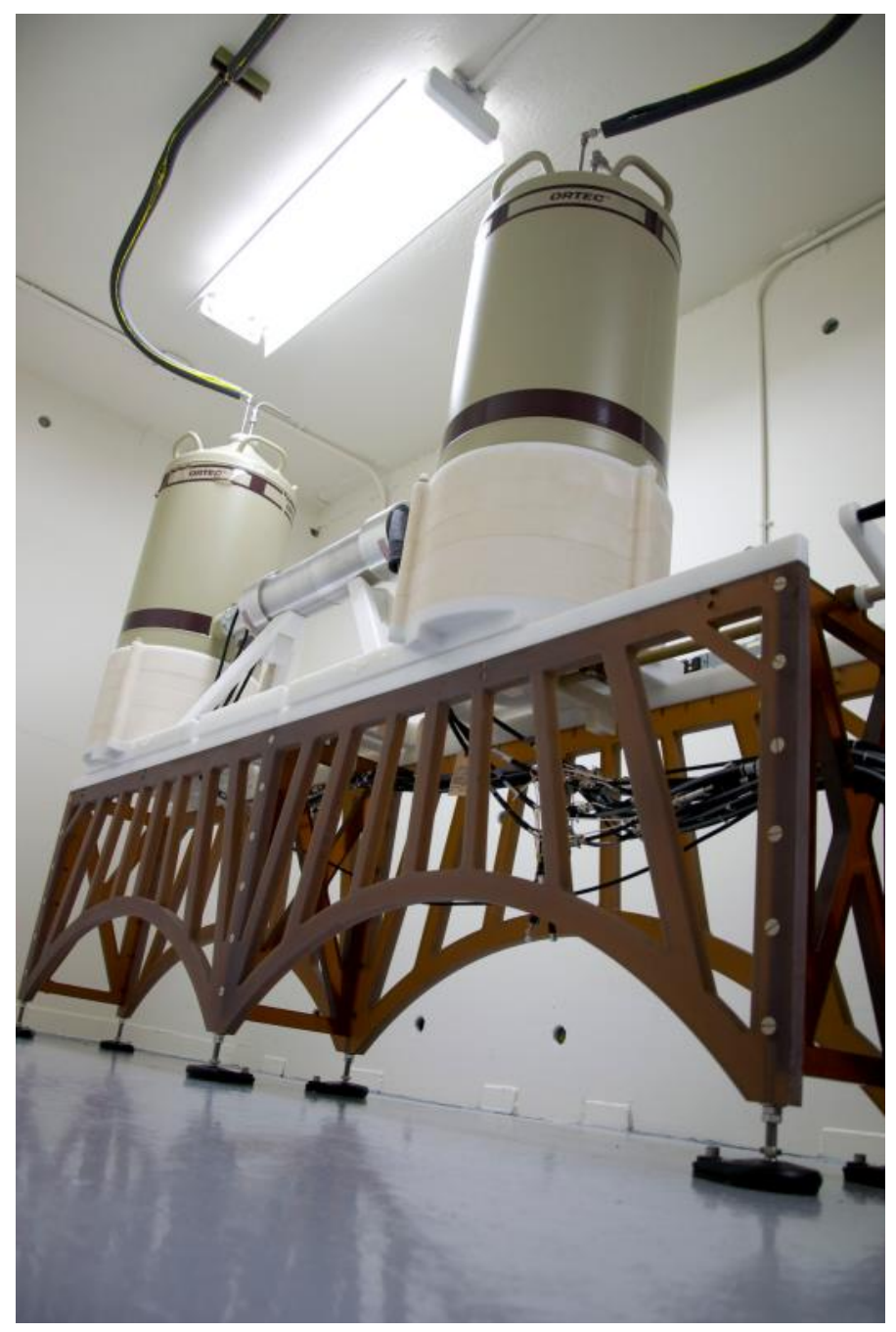

Figure 3: The clover system at LANL before the NaI(TI) shield had been installed 


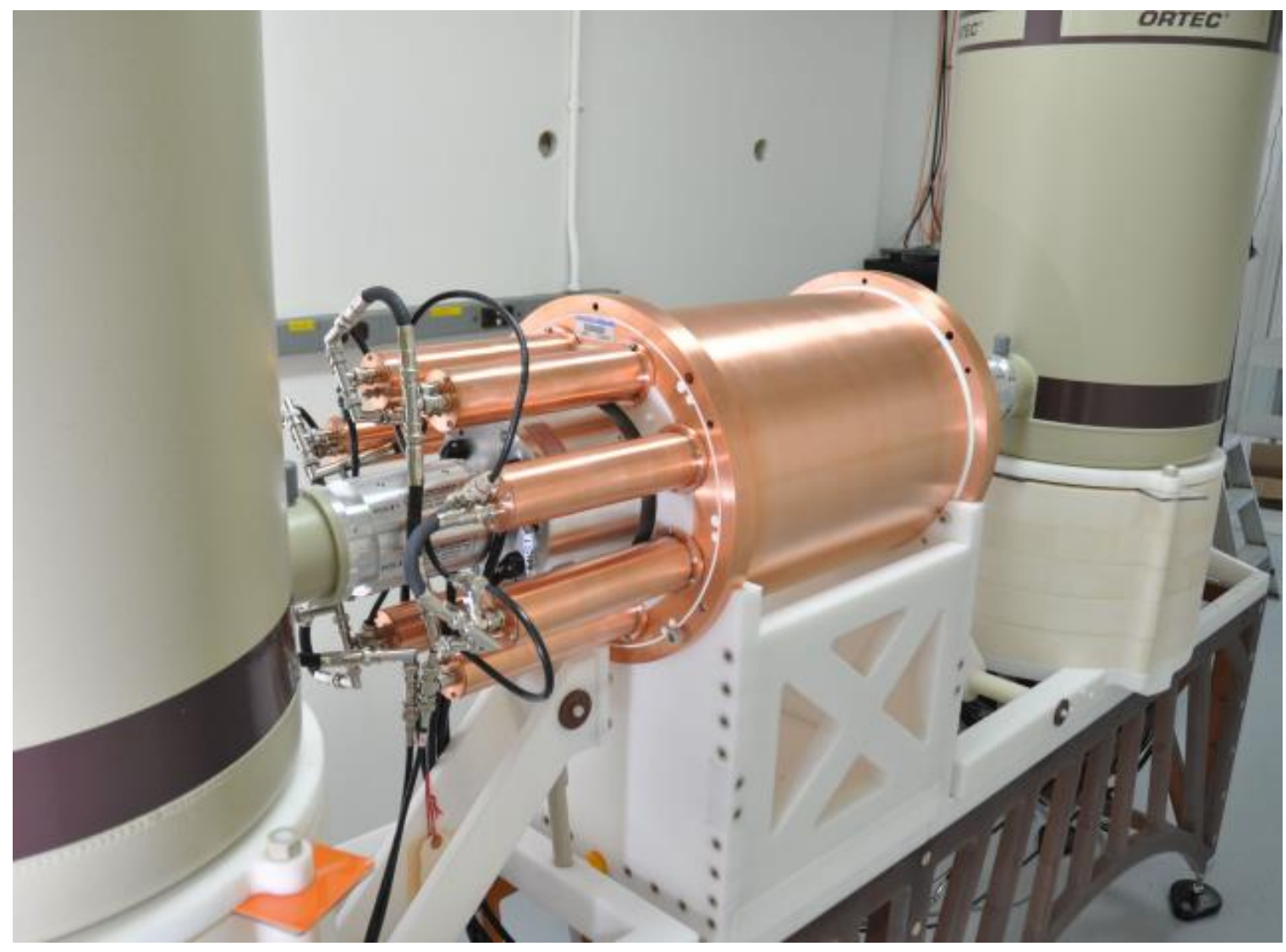

Figure 4: The clover system at LANL with the Nal(TI) shield installed

The preamplifiers for each HPGe crystal and the PMTs for each NaI(TI) quadrant are connected to XIA Pixie-4 $75 \mathrm{MS} / \mathrm{s}$ digitizers. The data is collected using list-mode and manipulated to produce various counting mode spectra. The crystals and PMTs are each connected to individual channels in the Pixie digitizers. There are a total of 16 channels being utilized in the digitizer system, four channels for each clover detector and eight channels for the $\mathrm{Nal}(\mathrm{TI})$ annulus. All modules are connected to a single control computer and are synchronized to the same clock to ensure proper timing for data acquisition. The modules have been tested and are synched to within one clock cycle ( $13 \mathrm{~ns}$ ) of each other.

The two clover detectors were given the names Misty and Kerri in order to distinguish them from each other. The names originate from the female US beach volleyball players Misty May-Treanor and Kerri Walsh-Jennings, who won gold medals in the 2004, 2008, and 2012 
summer Olympic games. These names will be used throughout the rest of this work to identify the individual clover detectors.

\subsection{Irradiated Uranium Analysis}

Gamma radiation from fission products produced by the irradiation of enriched uranium foils in the MIT research reactor has been measured to show the usefulness of the system for the analysis of nuclear forensics samples. The fission product distribution resulting from these irradiations can be analyzed to find general signatures that are important to forensics analysis [16]. By analyzing uranium samples that have been irradiated, the above concepts were used to demonstrate how the gamma-spectrum from the fission products evolves throughout the course of six months. Of the numerous fission products produced by irradiating uranium, many will have decayed away within 24 hours. As these fission products decay through their respective decay chains, other radionuclides will often grow in and subsequently decay away. Measuring fission products can give clues as to the original composition of the sample, the neutron energy spectrum that the sample was exposed to, and the age of the sample being analyzed. 


\section{Chapter 3: Literature Review}

In 1998, Duchene et al. introduced the clover detector, including descriptions of its initial design, simulations, possible uses, and different modes of operation [12]. The use of clover detectors has steadily increased and has shown to be useful for a variety of applications [17-19]. As seen in the above references, clover detectors have the capability of making large HPGe detectors that are faster and more efficient than would otherwise be possible.

In 1999, researchers from Lawrence Berkeley National Laboratory created a large array of HPGe detectors that are used for tracking gamma-rays paths and interactions over a $4-\pi$ geometry [17]. They used analog-to-digital convertors to convert the collected charge signal from the preamplifier to a digital signal that could be processed and time-stamped for analysis after the count. Since then, fast digitizers in conjunction with HPGe detectors have been in use for a variety of projects including coincidence-summing event recovery [18], the discovery of ${ }^{238} \mathrm{Th}$ [19], and fission product measurements [20].

Digitizers have been used in conjunction with gamma-gamma coincidence in projects such as identifying cosmogenic ${ }^{22} \mathrm{Na}$ [21], characterizing uranium isotopic fingerprints [22], and testing instruments for gamma-gamma coincidence infield measurements [23]. In 2008, Tomlin, Zeisler, and Lindstrom gave a very good overview of the use of gamma-gamma coincidence with digitizers in neutron activation analysis [24]. They irradiated bovine liver and showed that for the determination of $\mathrm{Ag}, \mathrm{Co}, \mathrm{Cs}, \mathrm{Se}, \mathrm{Fe}$, and $\mathrm{Sc}$, the peak-to-continuum ratio was improved dramatically by utilizing gamma-gamma coincidences.

In 2004, Dababneh et al. gave an overview of performing gamma spectroscopy using two clover detectors in close proximity including benefits and drawbacks [14]. Specifically, they examined the usefulness of treating each crystal as a separate detector in "Singles" mode, as well as the usefulness of "Add Back" mode, where each clover is treated as a single large detector. They also mentioned the possibility of examining summing effects and cascade events using a gate on one crystal and Add Back mode on the remaining seven crystals. They also examined gamma-gamma coincidence for several nuclides, concluding that the efficiency of the detectors are reduced significantly, but is made up for by the reduction in continuum around the peak. When using gamma-gamma coincidence, it should be noted that higher energy photons which Compton scatter through the gated energy window will produce peaks in the 
gated spectrum of energy $E_{c}=E_{b}-E_{\text {gate }}$ where $E_{c}$ is the observed peak in the gated spectrum, $E_{b}$ is the full-energy of the Compton scattered photon and $E_{\text {gate }}$ is the energy being gated on. Another difficult part of characterizing the system is obtaining the absolute full-energy peak efficiency of the system for the coincidence spectrum. Because angular correlations of cascading gammas are different for each nuclide, the efficiency may need to be determined for each individual nuclide [25]. This can be done by comparing the gated coincidence spectrum with the singles spectrum for each nuclide. Dabebneh et al. then go on to examine an experimental way of determining the coincidence summing correction factor, using a gated coincidence spectrum from one crystal and looking at the energies deposited in the other seven crystals. 


\section{Part 2: Initialization of the Detector System}

\section{Chapter 4: System Settings}

\subsection{Pulse Shape Settings}

To properly determine the energy deposition from a photon interaction within a detector, the pulse generated by the preamplifier must be shaped to improve the precision and fidelity of the algorithm to pick off the pulse height. Some of these analyzable forms include Gaussian, trapezoidal, or triangular shapes [4]. For systems in which the charge collection time is dependent on the position of the interaction in the detector, problems with the ballistic deficit can occur with certain pulse shape algorithms. The ballistic deficit represents the amount of charge on the preamplifier capacitor that is lost due to the time constant of the RC circuit [4]. If the time constant were infinite, the charge collected on the capacitor would remain constant, and the full charge would be collected. However, this would not allow for subsequent pulses to be analyzed correctly; therefore, the charge on the capacitor should be allowed to leak away slowly to allow the preamplifier output to return to the baseline. The magnitude of the ballistic deficit for a given pulse is dependent on the charge collection time. Because this can depend on the location of the interaction within the detector, the pulse shaping algorithm should allow for varying ballistic deficits. Shaped pulses with flat tops, such as trapezoids, will minimize the problem of ballistic deficits in pulses [4]. The downfall of trapezoidal filters is that they generally require longer shaping times than other filter shapes. The shaping time of the electronics is defined as the length of time per event that the preamplifier needs to collect charge so that the pulse can be put through the shaping algorithm.

The Pixie-4 digitizers in the LANL system use a trapezoidal shaping function [15]. The voltage on the preamplifier capacitor is read every $13.33 \mathrm{~ns}$ and stored internally. These points are stored and continuously analyzed for pulse-height by the digitizer until an event is detected. Once detection occurs, the pulse-height stored information in the digitizer is extracted to the external file. The shaping algorithm relies heavily on two user-supplied inputs: the Energy Rise Time and the Energy Flat Top. Because the energy deposition in the detector is approximately proportional to the amount of charge collected in the semiconductor, all shaping algorithms are designed to calculate the preamplifier pulse height. The digital trapezoidal shaping filter does 
this by summing a certain number of points at the height of the pulse and subtracting the sum of the same number of points at the baseline. The difference of these values is output to the multichannel analyzer (or equivalent) for energy binning. The Energy Rise Time setting determines the number of points that are summed at the pulse height/baseline. The longer the Rise Time, the more points are used to determine the difference between the pulse height and the baseline. If the Energy Rise Time is too short, then the random fluctuations due to noise can affect the peak resolutions in the detector. If the Energy Rise Time is too long, then a significant amount of dead-time per pulse will be incurred in the detector [4]. The Energy Flat Top determines the number of points separating the baseline segment from the pulse height segment. This is because the rising edge of the pulse is indicative of neither the baseline nor the pulse height, so it should not be included in either calculation. A longer Flat Top corresponds to more points separating the end of the baseline segment from the start of the pulse height segment. If the Flat Top is too long, excess dead-time will occur in the system and the calculated pulse amplitude will not be correct. This is because the pulse height will be calculated after some of the charge has dissipated from the capacitor.

Figure 5 shows how the trapezoidal shape is extracted from the preamplifier pulse output. For each image in the Figure, the green boxes correspond to the Energy Rise Time and the red box corresponds to the Energy Flat Top. The blue diamonds represent the digital output of the preamplifier and are output every $13.33 \mathrm{~ns}$. When an event occurs, the preamplifier voltage rises quickly and then slowly dies away based on the RC time constant of the preamplifier. The shaping filter operates by summing all the points in the left-most green box and subtracting this from the sum of the points in the right-most green box. The points within the red box are ignored. The difference between the green boxes is then recorded in the shaping algorithm memory. As the preamplifier continues to output data, the shaping amplifier continually determines and stores this difference. As can be seen in Figure 5 (A), the difference between the two boxes is close to zero. When an event occurs and the green boxes start encompassing the rising edge of the pulse, the sum of the points in the right-most green box becomes greater than that of the left-most green box, as seen in Figure 5 (B). Eventually, the difference between the boxes will reach a maximum, when the entire rising edge of the pulse is encompassed by the red box as shown in Figure 5 (C). As the shaping filter continues, the 
output of the filter will decrease from the maximum as the left-most green box starts incorporating the rising edge, as shown in Figure 5 (D). The resulting output is a trapezoid with the height being proportional to the deposited energy in the detector, which can be observed in Figure 6. The output of this filter shaping procedure is continuously calculated and recorded, regardless of whether the system has determined if an event has occurred. This data is recorded in a circular loop that overwrites itself when it determines that the earliest data point is not needed because no event triggers have been issued. This earliest data point is then overwritten with the most recent data point [15].
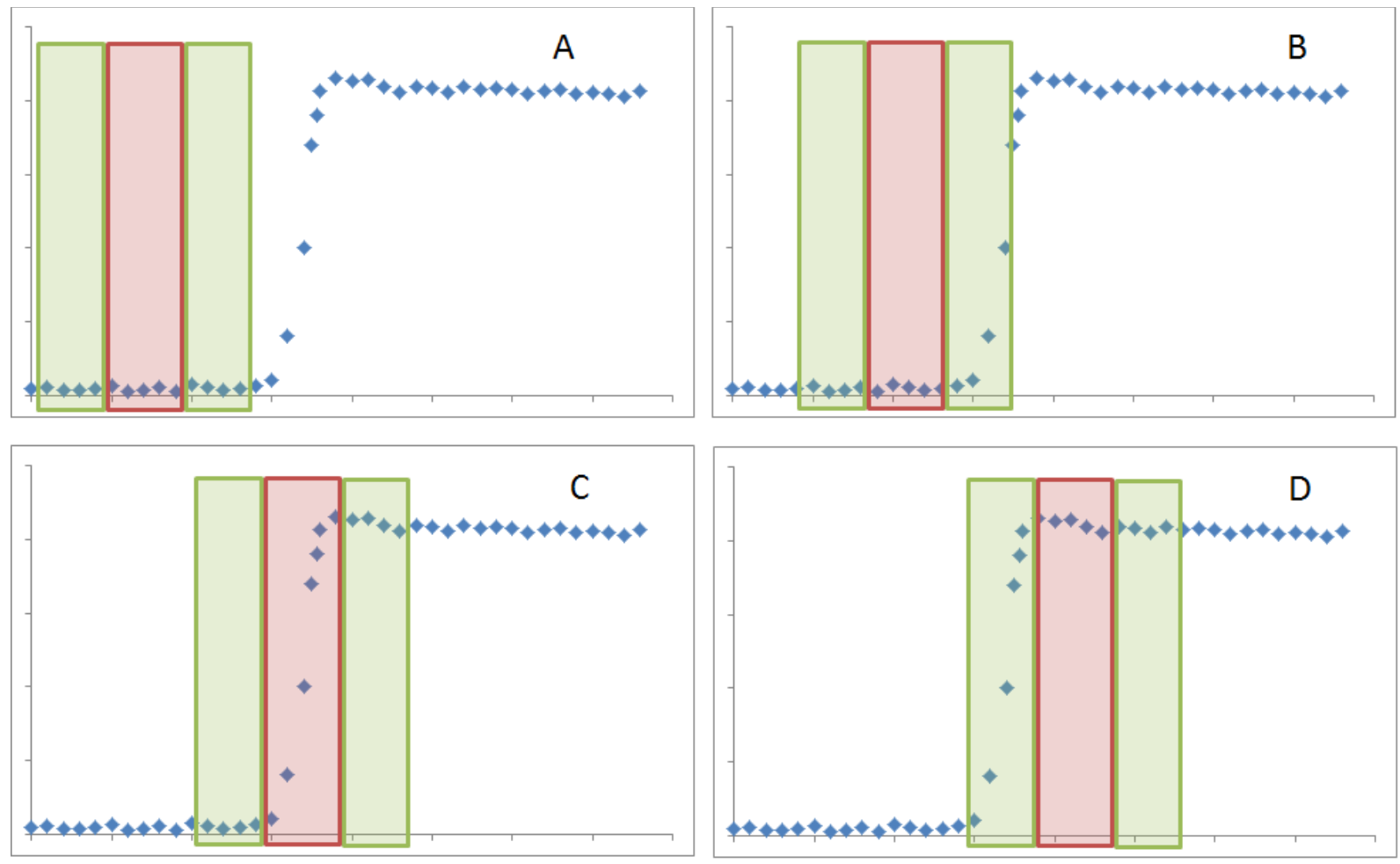

Figure 5: Demonstration of shape filter and trigger algorithm for digitizer

If an additional event occurs while an event is going through the filter shaping procedure, the output from the preamplifier will be the result of two photon interactions, and the shaping filter will be unable to distinguish between the two events as can be seen in Figure 6. Therefore, if two events are detected within the shaping time, both events are rejected as pileup. Longer shaping times give a higher probability of pileup events occurring during the count time; however, if the shaping time is too short, the shaping filter will not perform optimally and poor spectral resolution can occur. The priority for optimizing the shaping 
settings in the system at Los Alamos National Laboratory was to yield the highest quality data (optimal spectral resolution). Therefore, the combination of Energy Rise Time and Energy Flat Top settings were determined such that the best spectral resolution was obtained with a secondary emphasis placed on the throughput of the system. In other words, the shaping parameters were determined to be the shortest parameters that yielded the highest resolution for all peaks in the spectrum.

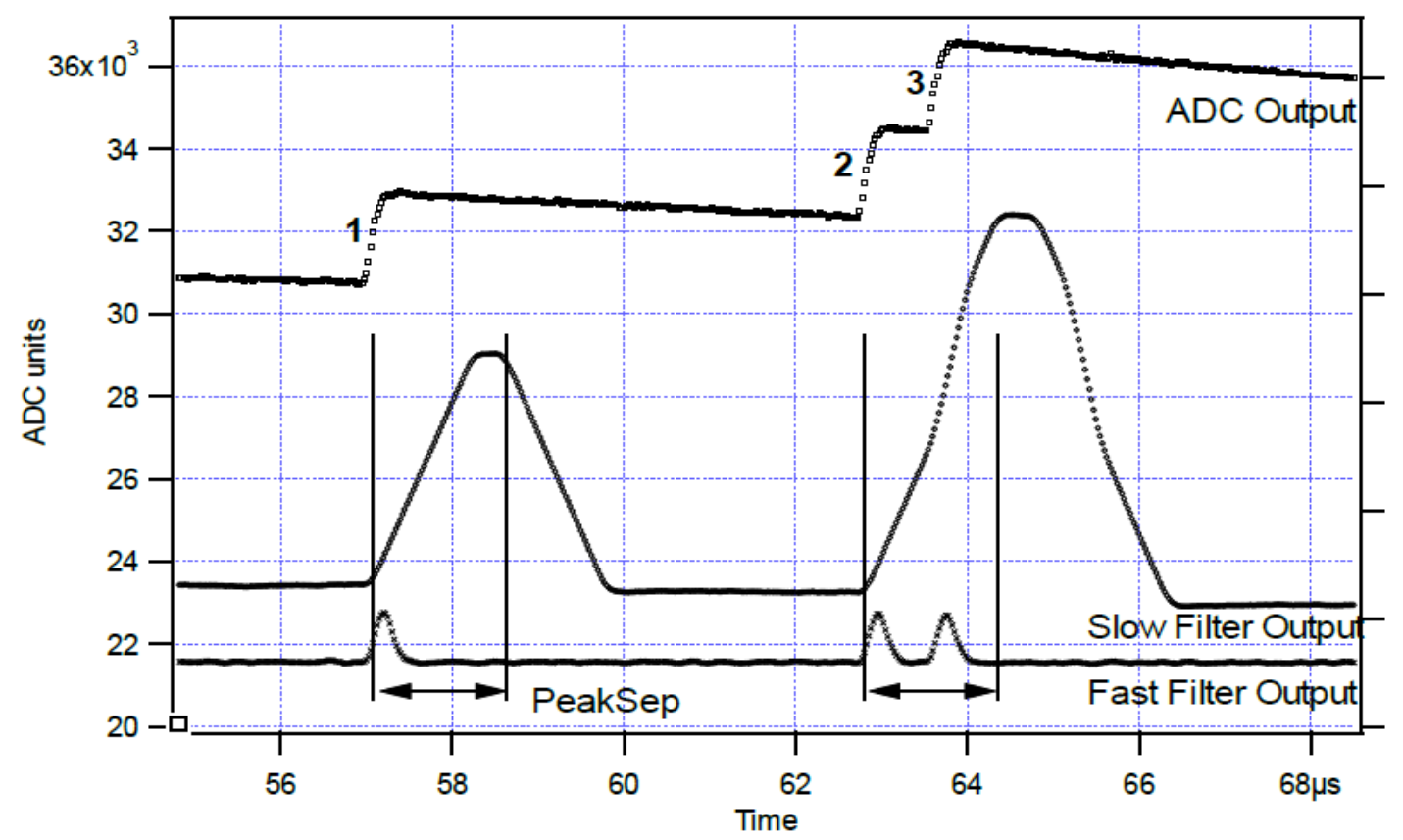

Figure 6: Representation of trigger and shaping procedure for Pixie-4 digitizers

Figure 6 also shows the output from the ADC. When an event occurs, the ADC output rises sharply as freed charge from the interaction is collected in the preamplifier, then decays slowly back to the baseline as the charge on the capacitor dissipates. This rate of decay back to the baseline is dependent on the RC circuit in the preamplifier. If an event occurs before the capacitor has fully discharged, the digitizer can correct the output back to the baseline by making use of the Tau setting. The shaping algorithm (given by the Slow Filter Output in the center of Figure 6) then creates a trapezoid with which it can pick off the pulse height. If two interactions occur within the shaping time of the system, depicted by events 2 and 3 in the Figure, the Slow Filter Output will produce a distorted trapezoid that is indicative of neither pulse. In this case, the Fast Filter Output, shown on the bottom of Figure 6, will produce two 
triggers within a short time span, indicating that the Slow Filter Output value will be incorrect. If two triggers occur within this shaping time, indicated by "PeakSep" in the Figure, then the system will realize that the pulse has piled up and both events are rejected. This minimum separation distance is defined in the system as the Energy Rise Time plus the Energy Flat Top. Pileup events may not be rejected if a second event occurs on the rising edge of the first event. In this case, the Fast Filter Output would stay above the threshold for both events, issuing a single trigger. When this occurs, the event is processed normally and the output of the shaping filter is not rejected.

To obtain the optimal settings, a program was written that took a 60 second spectrum of ${ }^{137} \mathrm{Cs}$ for different Energy Rise Time and Energy Flat Top combinations. The Flat Top was varied from $0.3 \mu$ s to $2.5 \mu$ s. Figure 7 and Figure 8 show the dependence of the Energy Flat Top setting for Kerri and Misty for a variety of Energy Rise Time settings. As can be seen in these Figures, the resolution does not depend very highly on the Energy Flat Top setting. The fullwidth at half-max (FWHM) of the $662 \mathrm{keV}$ peak from ${ }^{137} \mathrm{Cs}$ only fluctuated from 10-13 channels across the whole range of Energy Flat Top settings (where one channel is approximately 0.10 $\mathrm{keV})$, and a variety of Energy Rise Time settings. The Energy Flat Top may have more of an effect on higher energy photons, because the charge collection time will be higher due to more electrons being excited throughout the crystal. However, the Energy Rise Time appears to have the most significant influence on the energy resolution of the system. Because the Energy Flat Top does not appear to have much influence over the resolution of the spectrum peak, it was set based on qualitative observations of pulses in the oscilloscope. The value $1.2 \mu$ s was chosen by observing the time it takes for all pulses to rise fully and gives a conservative estimate of the width of the rising edge for all crystals in the system. 


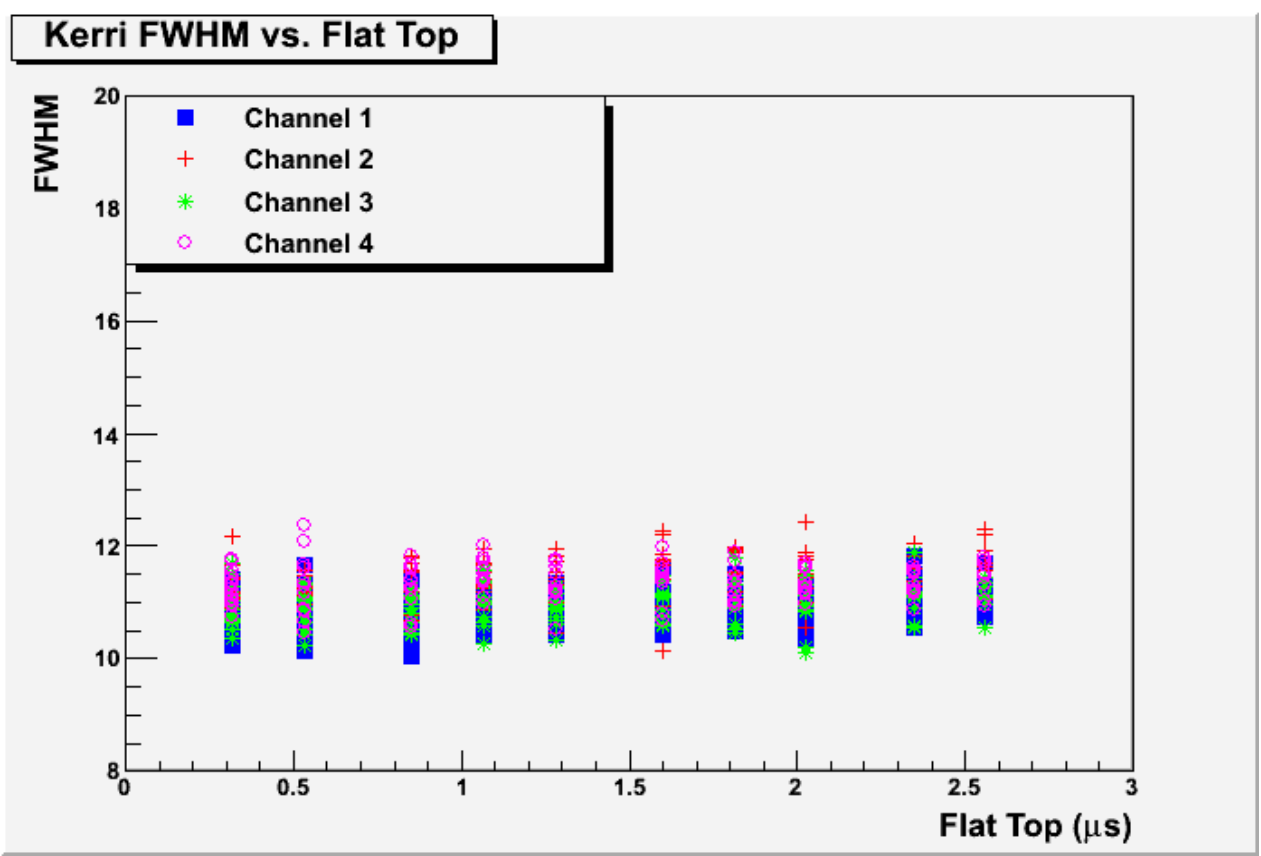

Figure 7: Dependence of peak resolution on the Energy Flat Top in Kerri

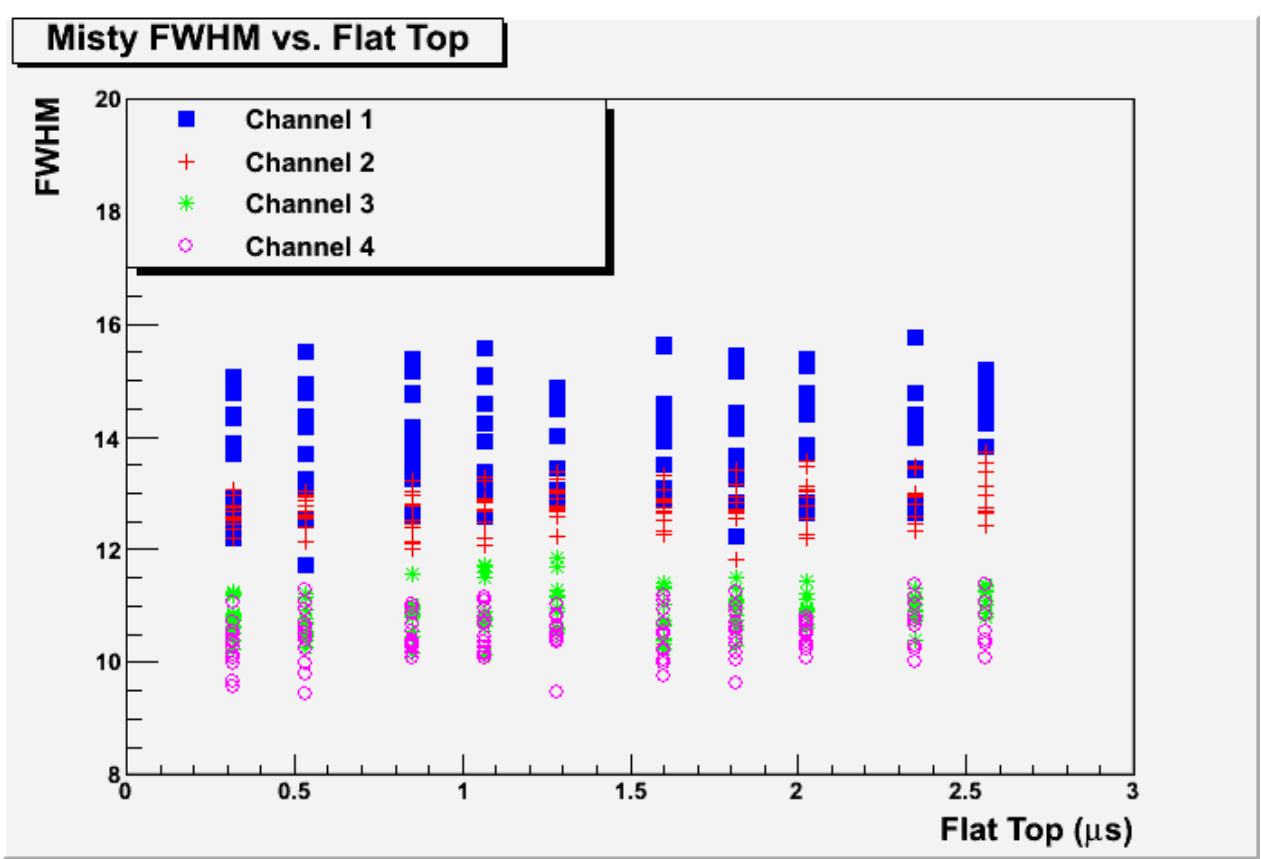

Figure 8: Dependence of peak resolution on the Energy Flat Top in Misty

To determine the optimal Energy Rise Time, the Energy Flat Top was fixed at $1.2 \mu$, and the Energy Rise Time was varied between $1.0 \mu \mathrm{s}$ and $50 \mu \mathrm{s}$. The results can be seen in Figure 9 and Figure 10. The peak in Figure 10 was further looked into, and it was found that a pulse with 
a period of about $5 \mu$ s was associated with Channel 1 of Misty. When the pulser was removed, the peak was no longer present. Observing the Figures, the resolution is poorest at the lowest Energy Rise Time and improves with increasing Energy Rise Time. However, at very high Energy Rise Time values, the resolution starts to get worse. This is because longer Energy Rise Time settings integrate too much noise, which results in poorer peak resolutions [26]. In order to maximize throughput while achieving the optimal spectral resolution, an Energy Rise Time of $12.0 \mu$ s was chosen for all HPGe crystals in the system. The same values were chosen for each crystal because the shaping times of simultaneous events within the same module can have an effect on the timestamp associated with that event and thus can affect how the system determines coincident events.

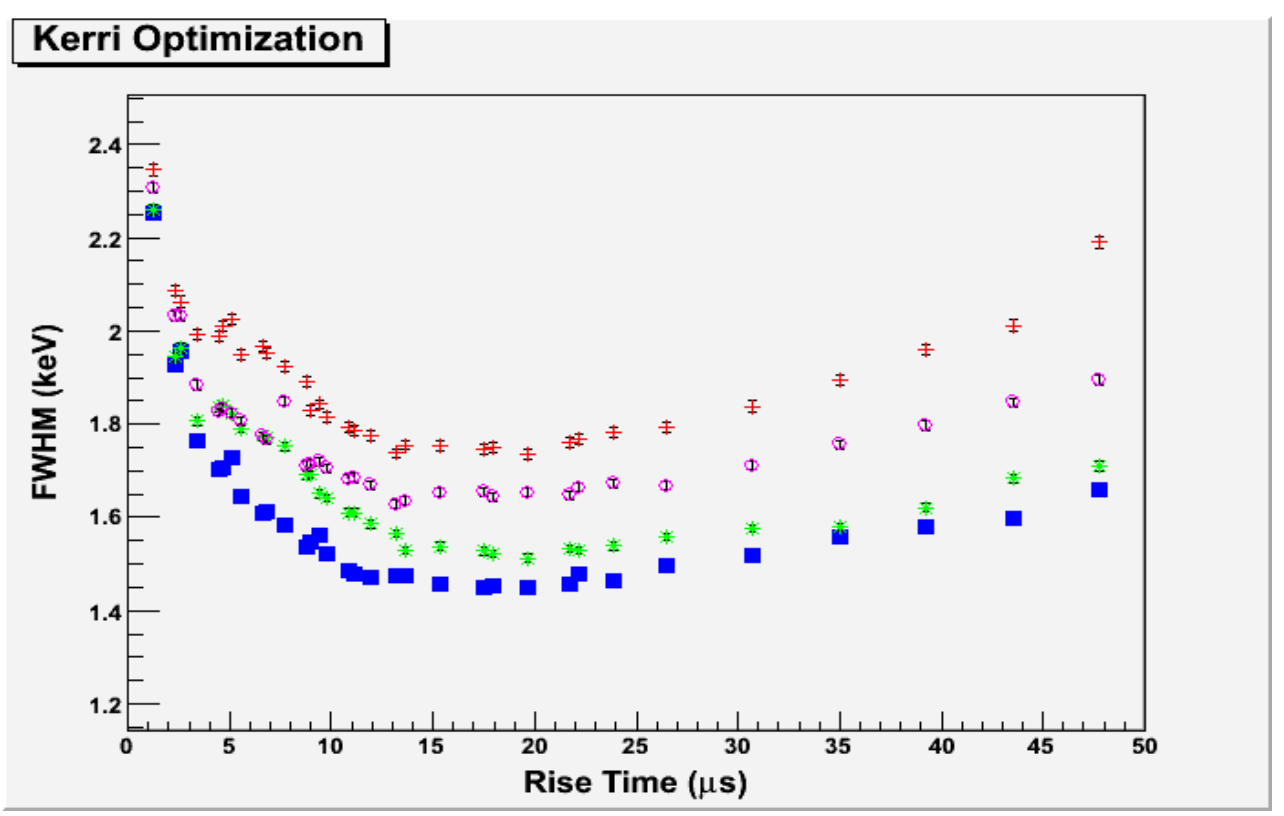

Figure 9: The dependence of peak resolution on the Energy Rise Time in Kerri 


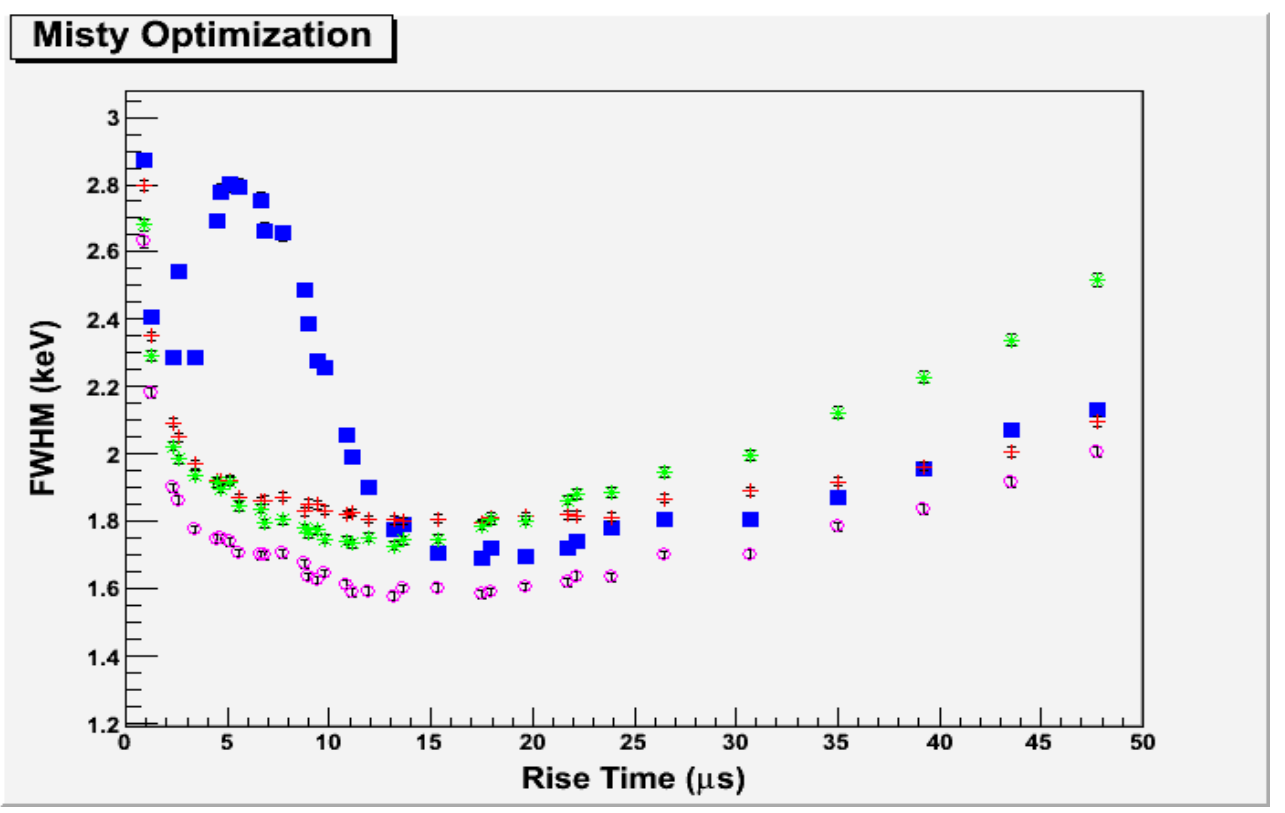

Figure 10: The dependence of peak resolution on the Energy Rise Time in Misty

Another setting that can influence the resolution of the system is the Tau setting. As a pulse is returning to the baseline, the digitizer is able to accept another event if it occurs after the shaping time of the previous pulse (if not, it would be rejected as a pileup event). Tau corrects the calculation of the pulse height by transforming the tail of the previous pulse onto the baseline, thus ensuring that the tail does not interfere with the pulse height calculation of the current event. The interface that comes with the Pixie-4 system is run in Igor. In this Igor program, there is a function that will automatically calculate Tau for a given pulse tail. The falling tail of a pulse can be defined by an exponential decay equation of the form:

$$
V=A e^{-\tau t}
$$

where

$V$ is the output of the preamplifier

$A$ is a constant depending on the pulse height

$t$ is time

$\tau$ is the value associated with the Tau setting 
The Pixie system comes equipped with Igor Pro - a piece of software that is equipped with many useful features for spectral analysis with this system. When a pulse is captured on the oscilloscope in Igor Pro, the software can fit the tail of the falling pulse and determine the proper value for Tau. This function was performed on a variety of pulses of different heights to ensure that the proper value for Tau had been obtained for each detector in all of the HPGe and $\operatorname{Nal}(\mathrm{TI})$ arrays.

\subsection{Energy Range Settings}

The energy range of the system is determined by the amount of gain applied to the preamplifier output signal. This is determined by the Gain $\left(V / V_{0}\right)$ setting in the system. The Pixie-4 gains can range from 0.97 to 11.25 , and is a multiplicative factor applied before the digitization of the preamplifier signal [15]. A lower Gain setting corresponds to a wider energy range of signals that can be processed and visualized on a gamma spectrum. If the Gain setting is too low, the analog-to-digital convertor (ADC) gain for the system (keV/bin) will be too high. This will cause peaks to be defined by a small number of channels, and spectral peak fitting algorithms can give inaccurate results. Another problem is that a portion of the low-energy side of the spectrum will be overwhelmed by the inherent system noise. If the Gain setting is too high, the desired energy range for the spectrum will not be fully included and high energy pulses will cause dead-time in the system due to overloading the voltage that can be handled by the shaping algorithms. This is because when a signal input is too high, the system will stop collecting data until the input voltage is back within an acceptable range [15]. Another problem from high Gain settings is that the noise in the system will increase along with the true signal.

For the types of samples being analyzed on this system, the primary photons of interest range from $50 \mathrm{keV}$ to $3000 \mathrm{keV}$. The Pixie-4 digitizers have the capability to create spectra with up to 65536 bins. A 65536 binned spectrum that has a maximum energy of $3000 \mathrm{keV}$ results in $0.0458 \mathrm{keV}$ per bin. However, even at the maximum Gain setting, this was not achievable. To maintain the desired energy range and a reasonable gain setting, the system was set to obtain spectra in only the first 32768 bins. A range of $3000 \mathrm{keV}$ across 32768 bins corresponds to $0.09155 \mathrm{keV}$ per bin. Because the system will be used in such a way that events from different crystals will be combined into one spectrum, it is very important to ensure that the conversion gain (keV / channel) for each individual detector crystal be as close as possible. If this is not 
precisely achieved, the resolution of the system will suffer. If the energy gains are vastly different, deformed peak shapes can occur, such as that seen in Figure 11.

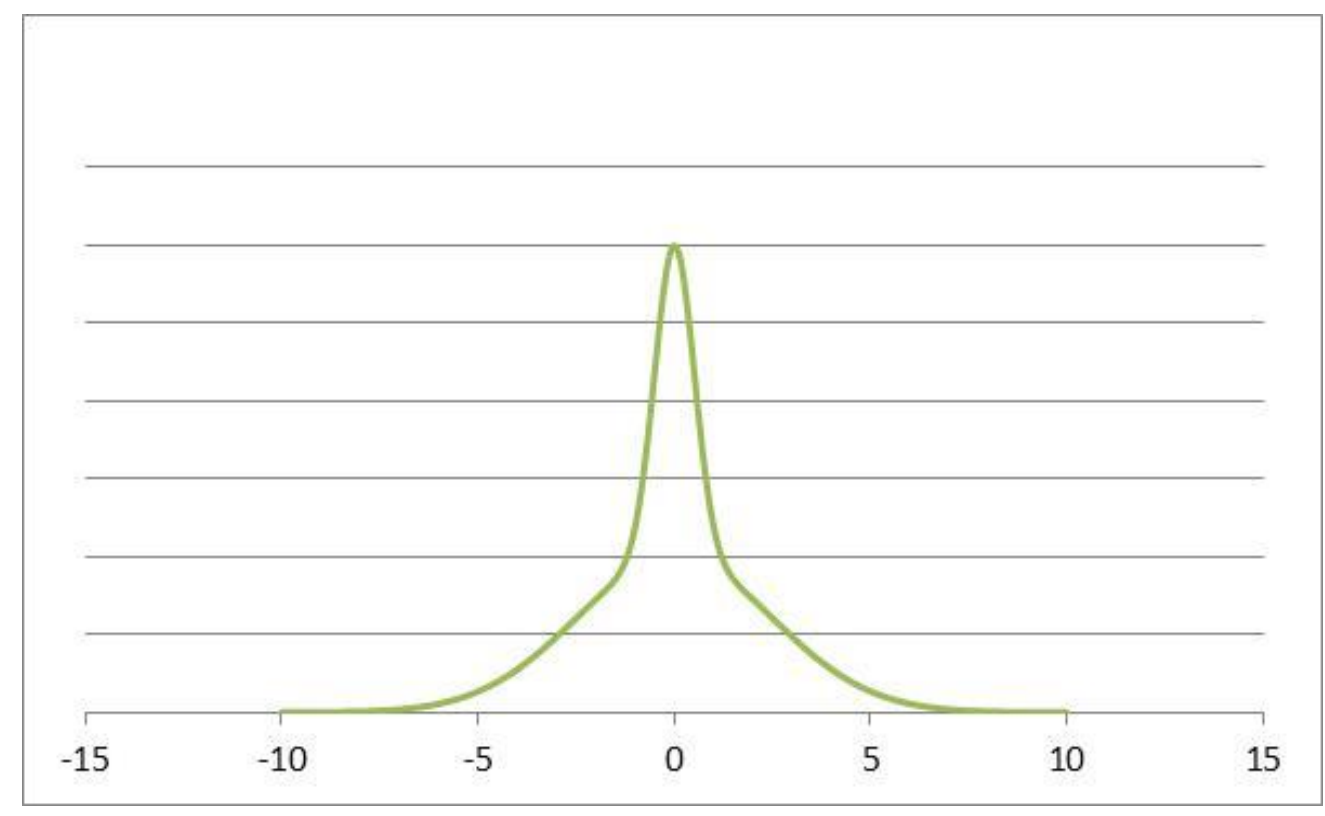

Figure 11: Possible deformed peak shape resulting from mismatched gain settings

To ensure that the system is equipped with the proper Gain setting, a computer program was created that empirically determines the Gain setting for each HPGe crystal such that the energy gain across the spectrum is matched to within $0.02 \%$. The program uses a ${ }^{152} \mathrm{Eu}$ source to provide gamma-rays at both low and high energies. Once the conversion gain falls to within $0.02 \%$ of $0.09155 \mathrm{keV} /$ channel, the Gain setting is kept the same. The program takes a 60-second spectrum with the user-provided Gain settings and identifies the centroid of the 121 $\mathrm{keV}$ and $1408 \mathrm{keV}$ peaks in the spectrum of each detector crystal. The number of bins between the centroids of these peaks is calculated, and the conversion gain across the spectrum is obtained. This number is compared with the expected 32768 channels/3000 keV, and the Gain setting is adjusted accordingly for each detector crystal until all crystals fall within $0.02 \%$ of $0.09155 \mathrm{keV} /$ channel. Variations within $0.02 \%$ were observed to be the smallest margin of error obtainable due to the inherent system drift. The new settings are then saved into an external settings file which can be transferred to the master system settings file. This ensures that the system is producing spectra with optimal resolution. Because channel 0 does not always correspond to $0.0 \mathrm{keV}$, the actual range of a given detector will not be exactly $[0,3000]$ $\mathrm{keV}$, but the difference between the high and low values of the range will be very close to 
$3000.0 \mathrm{keV}$. The fact that the minimum and maximum energies in each spectrum will be slightly different does not significantly affect the capability of the system, as peaks that occur at the low or high end of the spectrum range will not be used.

\subsection{Low-Energy Threshold and Trigger Settings}

The threshold and trigger settings determine which fluctuations in the preamplifier outputs should be regarded as actual events. Generally, fluctuations in the preamplifier outputs are either due to real events from radioactive sources, or are due to noise in the system [4]. Ideally, the spectrum should contain only the desired events from radioactive sources, and be free from noise. This is achieved by finding the optimal threshold and trigger settings for the system.

The Pixie-4 digitizers have two trigger settings, namely the Trigger Rise Time and Trigger Flat Top. The names of these settings are very similar to the filter shape settings (Energy Rise Time and Energy Flat Top) because the algorithms are very similar. The output of the trigger shaping procedure uses the exact methodology of the filter shaping procedure; however, the trigger settings are generally much shorter than the shape settings. The output of the trigger shaping procedure is compared with the threshold setting simply named Threshold, which is specified by the user. Once the output of the trigger shaping procedure crosses above the Threshold setting, a trigger is sent indicating that an event has occurred and the output of the filter shaping procedure will be recorded in the spectrum (unless a subsequent pileup event is detected as described in Section 4.1). When the output of the trigger shaping procedure crosses the Threshold, the digitizer will record the output of the energy filter shaping procedure exactly PEAKSEP time steps later and record the timestamp and pulse height value in the list-mode file. PEAKSEP is an internal variable determined by the filter shape settings (the Energy Rise Time and Energy Flat Top) - the exact value is the sum of the Energy Rise Time and the Energy Flat Top plus one time step (13.33 ns). Figure 6 from Section 4.1 shows the relationship between the energy filter and trigger filter.

Because the output of the trigger shaping procedure is directly compared to the Threshold parameter, the exact location of the low-energy cutoff observed in the spectrum depends on the Threshold as well as the Trigger Rise Time and the Trigger Flat Top. The output of the trigger shaping procedure is determined very similarly to the filter shaping procedure as 
shown in Figure 6. The sum of the data points in the left green box is subtracted from the sum of the data points in the right green box. If the difference between these sums is greater than the Threshold, then the event is flagged as a true event and the output of the filter shaping procedure will be recorded in the spectrum. If the Trigger Rise Time is increased, then the number of points being summed in the green boxes will increase. This has the effect of increasing the magnitude of the trigger output in the case of a true event and thus will be more likely be greater than or equal to the Threshold. This effectively lowers the low-energy threshold location in the spectrum even though the Threshold setting was unchanged. Increasing the Trigger Rise Time also has the effect of averaging out some of the noise events that may be affecting the system. Changing the Trigger Flat Top may also affect the location of the low-energy threshold in the spectrum. By increasing the Trigger Flat Top, the trigger shaping output will produce a greater maximum value in the case of a true event. Hence, the green boxes observed in Figure 6 will be further apart and the right-most green box will sum points that are further up the rising edge of the pulse. This also has the effect of "sharpening" the lowenergy threshold observed in the spectrum. If the Trigger Flat Top is too low, then the lowenergy threshold will appear as a gradual cliff as opposed to a sharp cutoff.

Caution should be taken in making the Trigger Rise Time and the Trigger Flat Top too large. The digitizers have an internal pulse pileup rejection technique that is designed to reject events that occur too close together. If two events occur too close together, then the filter shaping procedure will be unable to distinguish between the events and the resulting output will be affected by both events. To eliminate this effect, events are rejected if the trigger shaping output is observed to go above the Threshold twice before the filter shaping procedure is complete, as seen in the right part of Figure 6. However, if two events originating from different sources occur in the detector crystal before the trigger shaping output dips back below the Threshold, the events are not rejected and the digitizer will record an event corresponding to the sum of the energies of the events - a problem known as "random summing." Therefore, the Trigger Rise Time, Trigger Flat Top, and Threshold settings should be adjusted such that the lowenergy threshold observed in the spectrum is adequately sharp and no noise is observed in the spectrum, and keeping the Trigger Rise Time and Trigger Flat Top small enough to avoid significant random summing issues. The Threshold setting should also be set as low as possible 
to obtain the desired energy range, but also ensure that noise does not affect the spectrum. If the Threshold is too high, low-energy pulses may not be triggered as events and could pileup with higher energy events resulting in random sum peaks or poor resolution. This can also have an effect when calculating True Coincidence Summing factors. If the Threshold for the detector system is not carefully set up, then low-energy events could sum with coincidentally emitted photons and cause a decrease in the observed full-energy peak in the spectrum.

Noise generally appears in gamma spectra as a "cliff" of low energy events. This can be observed in Figure 12, which was taken from a spectrum with improper trigger and threshold settings. These effects can also be observed in a Time-to-Amplitude Conversion (TAC) spectrum. At LANL, noise contributions can cause "coincidences" to be observed between detectors with a delay of about $0.5 \mu$ s due to an unknown radiofrequency noise source. This is because the period of the noise is around $0.5 \mu \mathrm{s}$, causing the post-processing program to observe a coincidence between the two detectors. In order to achieve the correct settings, one-minute spectra were obtained using various threshold and trigger settings. The noise was compared between spectra and suitable values were chosen that eliminate the noise at the low energy. The effect of the Trigger Flat Top was not observed on the sharpness of the low-energy threshold in the spectrum. This effect should be further investigated and an optimal value should be determined for the system. The values chosen for this system were $0.080 \mu$ s for the Trigger Rise Time and Trigger Flat Top for the HPGe crystals. A value of $0.108 \mu$ s was chosen for the Trigger Rise Time and $0.0 \mu$ s was chosen for the Trigger Flat Top for the Nal(TI) photomultiplier tubes (PMTs). The Threshold parameter was set to be 12 for the HPGe crystals and 10 for the Nal(TI) PMTs. The Threshold parameter differs from a lower-level discriminator in that it does not cut off signals below a certain level. As mentioned above, the Threshold setting combined with the Trigger Rise Time and Trigger Flat Top settings determine the location and sharpness of the low-energy threshold observed in the spectrum.

\subsection{Other Settings}

In addition to the settings already mentioned, there are other settings that are important for proper analysis using this system. The Filter Range variable allows higher values for the filter shape settings by averaging more than one data point from the Analog-to-Digital Converter (ADC) before being introduced to the filter shaping procedure [15]. This allows for a 


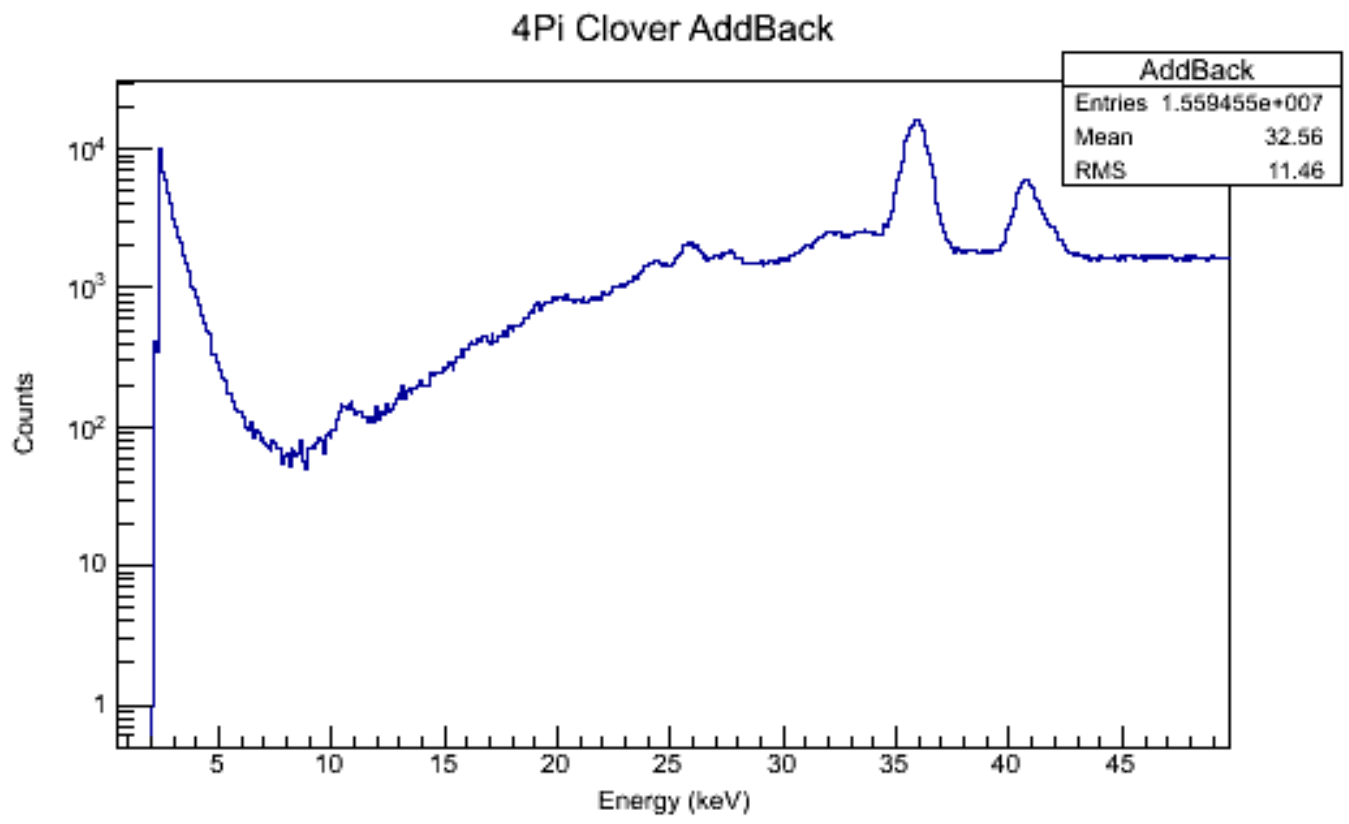

Figure 12: Low energy noise from poor threshold and trigger settings

wider range of shaping settings. The HPGe crystals for the clover detectors were selected to have $12.0 \mu$ s for the Energy Rise Time and $1.2 \mu$ s for the Energy Flat Top. These settings require the Filter Range to be set at 4 for the HPGe crystals. For the NaI(TI) settings, the Filter Range is set to 1 . This is because the $\mathrm{Nal}(\mathrm{TI})$ is being optimized for throughput and thus the shortest shaping time possible is used.

The Max Events setting determines how many events can be recorded before the current buffer should be dumped to the external memory file [15]. By specifying this setting, the buffer may be dumped before it is actually full, which will cause dead-time in the system. Because the system is being optimized for throughput as well as for resolution, this setting was set to 0 , which disables this feature of the digitizer and lets the buffer reach maximum capacity before dumping the data to the file. This reduces the dead-time in the system dramatically, especially for higher activity samples and does not affect the resolution of the system.

The Channel CSRA setting is a binary number that contains a number of flags. For all inputs, the Channel CSRA is set to a decimal value of 20 (binary equivalent: 0000000000010100). This ensures that all channels are considered "good" channels so they will contribute to the spectrum and it ensures that all channels can trigger an event. 
The Channel CSRC is another binary setting that allows for flags to be set on certain channels. For the Nal(TI) channels only, the Channel CSRC was set to a decimal value of 24 (binary equivalent: 0000000000011000), which turns off the pileup rejecter and lets the spectrum record events that were out of range. The Channel CSRC was set to 0 for the HPGe crystals. The pileup rejecter was turned off for the Nal(TI) annulus because pileup events are still indicative of true events and should be taken into consideration for Compton suppression purposes.

Other settings include the Trace Length, Trace Delay, Gate Window, and Gate Delay [15]. These settings should be set to 0.0 for all channels, because they can contribute to deadtime within the system [15]. These settings are for taking data with more features than required for this research. An example of an input parameter settings file is shown in Appendix 2. 


\section{Chapter 5: Modes of Operation}

The following sections describe the different counting modes that this system is able to obtain for analyzing samples. The optimal counting mode to use for analysis on a given nuclide in a sample depends on the decay scheme of the nuclide, the energies of the photons being analyzed, and other nuclides present in the sample which may interfere with the analysis being performed. When a sample is counted, the digitizer system creates a list mode file, in which the system records the timestamps and pulse heights of all valid events that were observed. A parsing routine created by Bob Rundberg and edited by Steven Horne was used to take this list mode file and create ROOT files containing various spectra. ROOT is a programming language based on $\mathrm{C}++$ that was developed by the physicists at the CERN laboratory in Switzerland for use

with data obtained using the Large Hadron Collider [27]. ROOT gives users the capability to handle large amounts of data quickly and to perform high-level functions necessary in many scientific fields. The functions applicable to this research include spectrum analysis, function fitting, histograming methods, and graphics/visualization. Because of the unique spectral features available, ROOT was chosen as the visualization programming language for this system. The following counting modes are a few of the spectra created in each ROOT file.

When an event is detected in a channel of a Pixie-4 module, any subsequent pulses in neighboring channels are collected based on the internal timing window for each module [15]. These detected pulses are then stored in the list-mode data file in the same event. Events can contain hits in one to four channels, depending on the number of channels that recorded a hit within the internal timing window for the module. This internal timing window is a preconfigured time interval based on the shaping time of the channels in the module [15]. The individual hits are each given their own timestamp which corresponds to the trigger time of the hit. The entire event is also given a timestamp, which is termed the "Event Timestamp." The parsing routine created by Rundberg and Horne looks for coincidences that occur between modules, such as between Misty and Kerri, or between a clover detector and an Nal(TI) channel. This parsing routine looks only at the Event Timestamp for each hit in order to compare them with the coincidence criteria for the counting mode. Some improvement in timing and peak resolution may be possible by using the individual channel trigger times as opposed to the module Event Timestamp for the hits. 
Because of the inherent uncertainty involved with the charge collection process, two events in coincidence may not be given the same timestamp by the system. This may be due to a number of factors which include the location of the interaction, differences in the detector crystals, and differences in cable lengths or impedances. A timing window must be established in order to determine coincidence criteria. To do this, a time-to-amplitude conversion (TAC) spectrum was created and observed for the optimal timing window. A typical TAC spectrum for coincidences between the two clover detectors is shown in Figure 13. A conservative window of $1.0 \mu \mathrm{s}$ was chosen for the timing window between the two clover modules, as well as between the clover modules and the NaI(TI) modules.

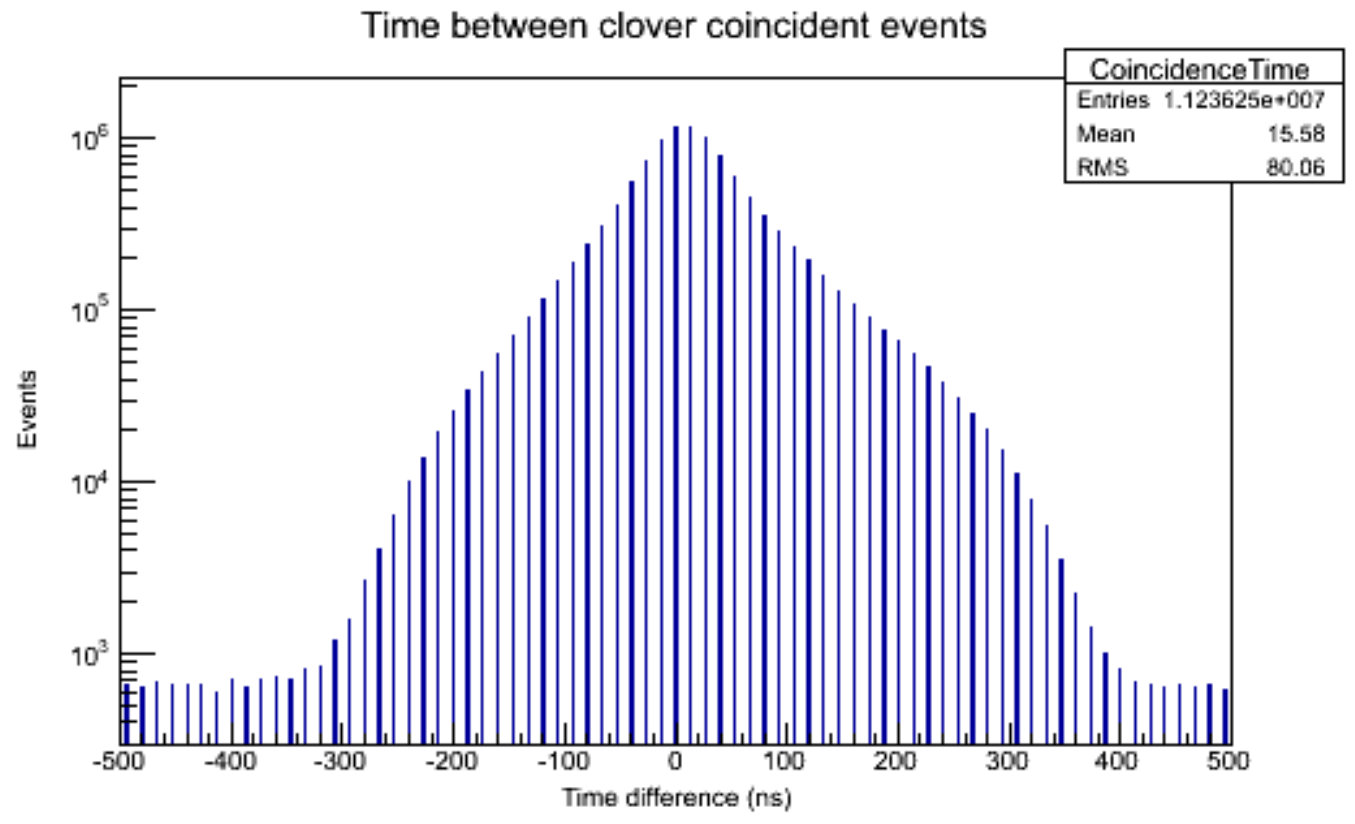

Figure 13: TAC spectrum for coincidences between the two clover detectors

\subsection{Add Back Mode}

Segmented detectors were created in order to simulate the performance of a large detector by using multiple smaller crystals in one cryostat [12]. In order to truly simulate the performance of large detectors, photons that deposit energy in more than one crystal, via Compton scattering or pair production, should have the sum of these multiple-crystal energy depositions binned in the spectrum. Add Back mode takes events that occur within a specified 
timing window and adds the energies of those events together, binning this summed energy in the spectrum.

As mentioned previously, a disadvantage of using large detector crystals is poorer timing resolution [12]. The average charge collection time is longer in bigger detector crystals [4], which results in degraded resolution in the obtained spectra. Both of these drawbacks can be reduced by using segmented detectors in Add Back mode. By adding together the energies of events that have Compton scattered between detector crystals, the effect is two-fold. First, the full-energy peak is populated with more events than otherwise would have been seen by the smaller detector crystals. Second, the Compton continuum is reduced, because many of these events get added into the full-energy peak. This may provide less background continuum for lower energy events in the spectrum. Relative to other modes of operation, Add Back mode is very susceptible to true-coincidence summing (TCS). The system itself is already very susceptible to TCS because of the close counting geometry, and using Add Back mode magnifies this disadvantage. If two or more photons are emitted simultaneously and the photons are detected in different crystals, Add Back mode will sum these energies together before binning them in the spectrum. Special care must be taken when analyzing radionuclides with cascading emissions in Add Back mode.

\subsection{Singles Mode}

Arguably the simplest and most intuitive way of operating a multi-crystal detector array is to simply add the contents of each spectrum and into a single spectrum. This way of taking data is termed "Singles" mode - named because each event recorded in the spectrum corresponds to a single interaction. The obvious advantage of taking data in this way is that the counting geometry of the detector is $\sim 4 \pi$, and therefore the count time needed to produce the same results about eight times less when compared to the results from a single detector. Singles mode is not as susceptible to true-coincidence summing as Add Back mode, because the solid angle in which true coincidence summing can occur is not as large for Singles mode. However, the Compton continuum will be much higher in Singles mode, because multiple Compton scatters involving more than one crystal will contribute multiple counts in the Compton continuum. 


\subsection{Compton Suppressed (Veto) Mode}

The large Nal(Tl) annulus gives a two-fold benefit. First, it provides passive shielding to background radiation, including NORM and cosmic sources. Evidence of this can be seen in Figure 14. The red graph (No Annulus) represents a background spectrum taken before the Nal(TI) annulus was installed. The green graph (No Veto) represents a background spectrum taken after the annulus was installed and having it serve as a passive shield only. Further reductions can be seen by the blue graph (Veto) where Compton suppression was incorporated into the background spectrum. Second, and more importantly, the annulus gives the capability of producing Compton-suppressed spectra. This involves using the annulus as an active shield. In this mode, an event is only binned in the spectrum if it did not coincide with an event recorded in the $\mathrm{Nal}(\mathrm{TI})$ annulus within a certain timing window. The purpose of this is to lower the Compton continuum by suppressing Compton scattered events that deposit a partial amount of the energy in the HPGe detector and scatter into the annulus (or deposit energy in the annulus and scatter into the HPGe detector). Further investigation of the background of the clover system at Los Alamos National Laboratory has been performed elsewhere [28].

The advantages of this Compton suppressed mode include a lower Compton continuum in the low-energy portion of the spectrum. This mode can be operated in Add Back mode or in Singles mode at the user's discretion. Veto Mode also can help researchers discriminate between overlapping peaks. If two peaks occur at around the same energy, but one of the peaks is the result of a photon that comes from a cascading emission, this peak will be partially suppressed in Veto Mode and the other (presuming it originates from a singlet emission) will retain the vast majority of its counts. However, the suppression of cascading emissions could also be a disadvantage, as this will decrease the full-energy peak area of possibly desirable peaks. Therefore, Compton suppression should be used judiciously. 


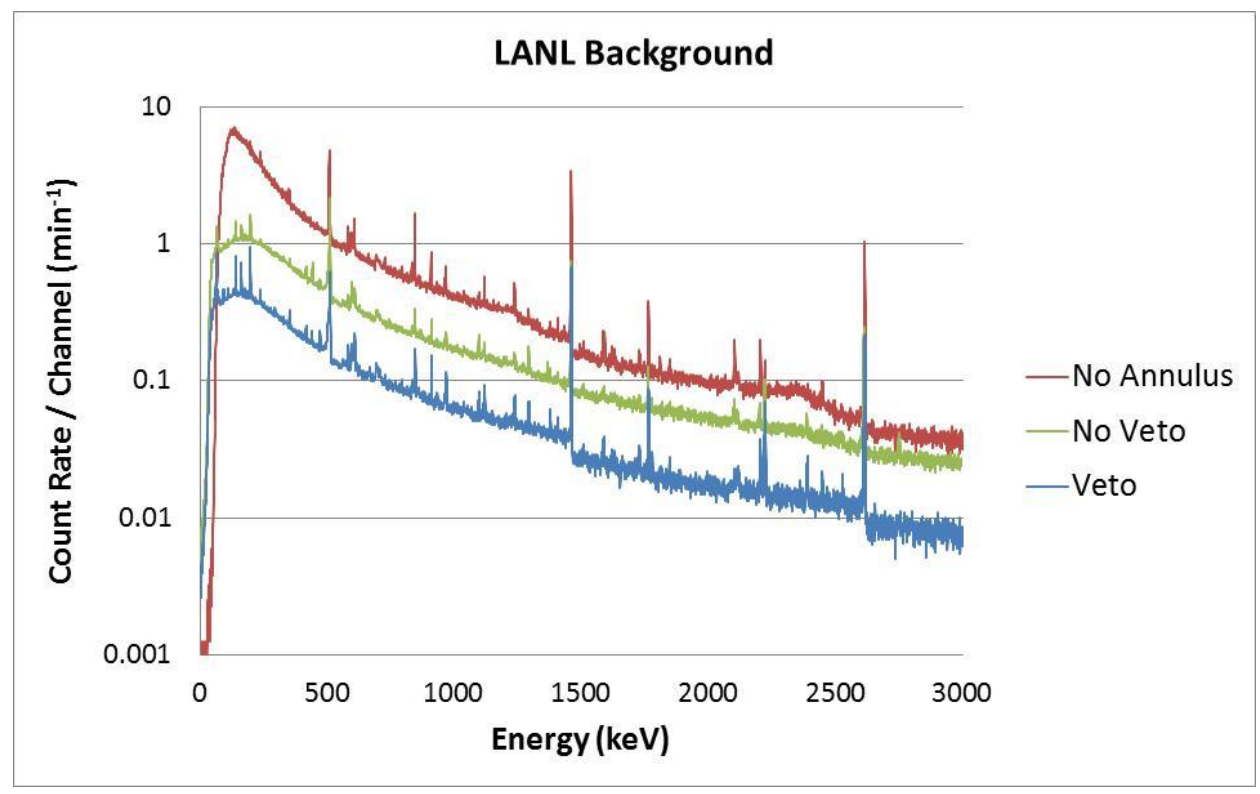

Figure 14: Background spectra from Los Alamos, NM

\subsection{Gamma-Gamma Coincidence Mode}

One of the less common, but potentially more powerful, modes of operation is gammagamma coincidence. This mode generates a two-dimensional plot, such as that seen in Figure 15. Both detectors are observed individually in Add Back mode (using 4-crystal Add Back, as opposed to 8-crystal Add Back used in the traditional Add Back mode). When two events are observed in the two clover detectors within a specified timing window, the energies of the two events are recorded in the two-dimensional spectrum. Figure 15 shows the gamma-gamma coincidence spectrum of ${ }^{60} \mathrm{Co}$. There are two spots on the spectrum with a large number of counts, which correspond to the $1173 \mathrm{keV}$ photon in Misty being detected in coincidence with the $1333 \mathrm{keV}$ photon in Kerri and vice-versa. The horizontal and vertical lines extending from these two spots correspond to full-energy depositions of one photon being detected in coincidence with a Compton scatter event of the other cascading photon. The diagonal line passing through both true-coincidence spots corresponds to a double backscatter event, in which both photons deposit some of their energy in one detector and scatter into the other detector where they deposit the remainder of their energy. This double backscatter event, in 
which all the energy is deposited between the two crystals, will always add up to the sum of the cascading photons $-2506 \mathrm{keV}$ in this case.

To create a spectrum that can be analyzed with Genie 2000, a projection of the twodimensional plot should be created. This essentially uses one of the detectors as an energy gate

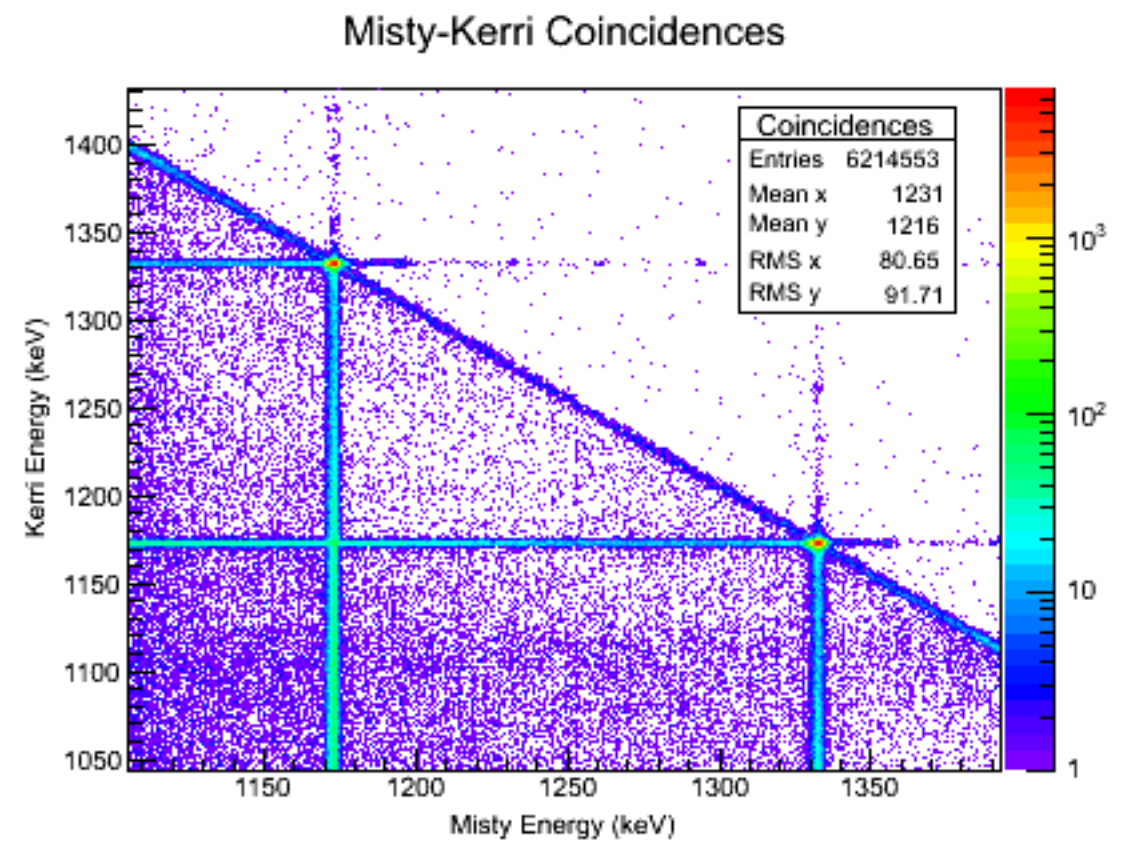

Figure 15: gamma-gamma coincidence plot of Co-60

and shows the spectrum of the other detector as a one-dimensional plot. The energy gate should be set such that the majority of the peak width is contained in the gate. This can be determined visually from the two-dimensional plot by observing the width of the continuum extending from the two-dimensional peak. A magnified portion of Figure 15 is shown in Figure 16. From this Figure, it can be observed that the $1173 \mathrm{keV}$ photon extends over about nine bins in Misty. Therefore, when gating on the $1173 \mathrm{keV}$ peak, the gate should extend across nine bins to fully incorporate all coincident counts. The resulting one-dimensional spectrum is shown in Figure 17. 


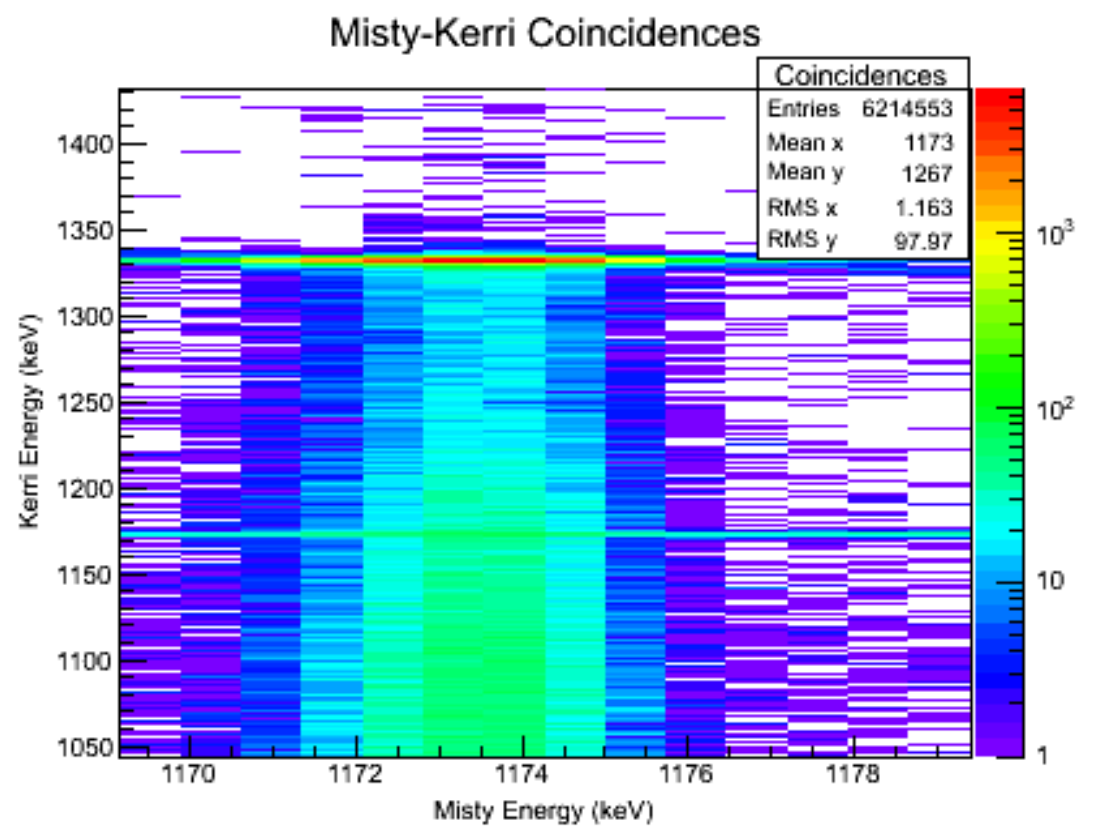

Figure 16: Magnified view of Co-60 2-Dimensional Spectrum

This method can be very powerful in determining low-level concentrations of nuclides in the midst of other nuclides with high gamma-emission rates. If the less abundant nuclides have strong cascading emissions, gamma-gamma coincidence may isolate these nuclides and give lower detection limits as opposed to other counting modes. Gamma-gamma coincidence mode could also be useful in determining higher energy photons by looking at the 511 keV annihilation photon in coincidence with a single-escape or double-escape peak. This could be useful for example if a high-energy peak is interfered by summing-in from lower energy photons emitted in cascade. The main disadvantage of using gamma-gamma coincidence mode is that the number of events detected per coincident-pair emission is much lower than the number of events detected per single gamma-ray emission. Therefore, the peak areas in this mode of counting will be much lower than those observed in non-gated spectra. The efficiency of the system for a given coincident-pair becomes $\varepsilon_{1} \varepsilon_{2}$, where $\varepsilon_{1}$ is the full-energy peak efficiency of one photon in one of the detectors (four-crystal Add Back mode efficiency) and $\varepsilon_{2}$ is the fullenergy peak efficiency of the other photon in the other detector (once again, using four-crystal Add Back mode efficiency). 


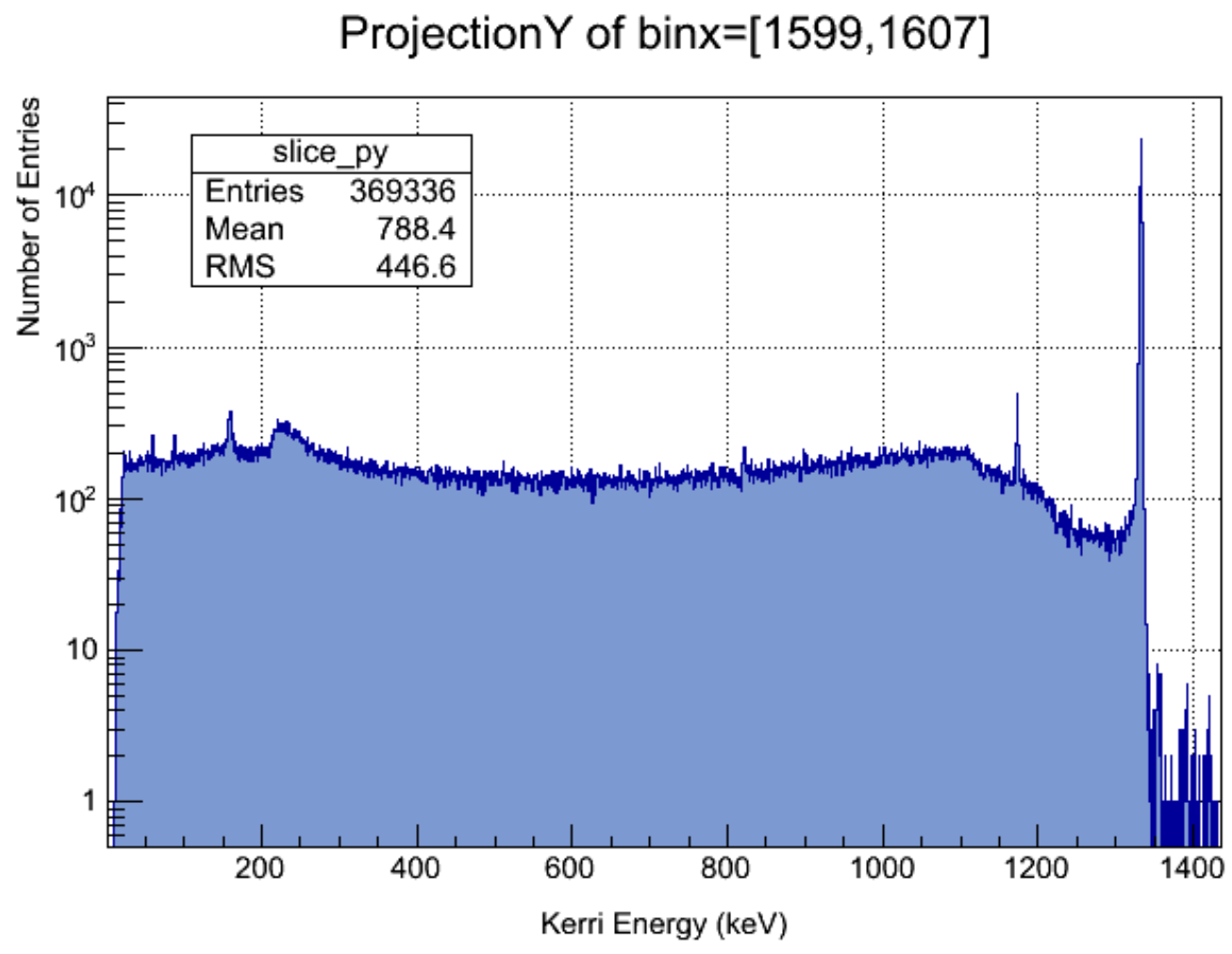

Figure 17: Co-60 gamma-gamma coincidence Spectrum Gated on $1173 \mathrm{keV}$ 


\section{Part 3: Characterization of the Detector System}

\section{Chapter 6: Peak-to-Total Ratios}

The clover detector system at LANL is to be used in a close-counting geometry in order to count samples in a near $4 \pi$ geometry. In Add Back mode, the solid angle of the system is close to $4 \pi$. Because of this, the system is very susceptible to true-coincidence summing (TCS) [13]. This occurs when two or more gammas are emitted simultaneously from the sample, and the system detects more than one of the gammas emitted, either in part or in whole. Because the timing resolution of the system is on the order of nanoseconds and the time between cascading emissions is generally less than one nanosecond, the system will combine coincident gamma interactions into one event. In Singles mode, this will occur only for interactions taking place in the same crystal; for Add Back mode, this will occur for any interactions observed in any crystal. When observing nuclides that emit cascading gammas, TCS must be corrected for unless the comparator method is being used. There are a variety of methods to correct for TCS, including individual nuclide calibrations, Monte Carlo simulations [29], and total efficiency measurements using peak-to-total methods $[13,30]$. This work will use the peak-to-total method to correct for TCS. It should be noted that true-coincidence summing can be helpful if two low energy gamma-rays sum to a higher energy where the underlying continuum and interferences are significantly lower.

\subsection{Samples Used in P/T Ratio Determination}

The peak-to-total method involves taking gamma spectra of single, or nearly single, gamma emitters and finding the ratio of the counts in the full-energy peak to all the counts attributable to the nuclide [30]. The sources used for this consisted of ${ }^{73} \mathrm{As},{ }^{241} \mathrm{Am},{ }^{109} \mathrm{Cd},{ }^{57} \mathrm{Co}$,

${ }^{113} \mathrm{Sn},{ }^{85} \mathrm{Sr},{ }^{137} \mathrm{Cs},{ }^{54} \mathrm{Mn}$, and ${ }^{65} \mathrm{Zn}$. The energies associated with these sources can be seen in Table 1. 
Table 1: Samples used in peak-to-total ratio determination

\begin{tabular}{|c|c|}
\hline Nuclide & Energy (keV) \\
\hline${ }^{73} \mathrm{As}$ & 53.440 \\
\hline${ }^{241} \mathrm{Am}$ & 59.5412 \\
\hline${ }^{109} \mathrm{Cd}$ & 88.04 \\
\hline${ }^{57} \mathrm{Co}$ & 122.0614 \\
\hline${ }^{113} \mathrm{Sn}$ & 391.690 \\
\hline${ }^{85} \mathrm{Sr}$ & 514.007 \\
\hline${ }^{137} \mathrm{Cs}$ & 661.657 \\
\hline${ }^{54} \mathrm{Mn}$ & 834.848 \\
\hline${ }^{65} \mathrm{Zn}$ & 1115.546 \\
\hline
\end{tabular}

${ }^{73}$ As decays $100 \%$ by electron capture to ${ }^{73} \mathrm{Ge}$ [31], hence there is some probability of coincidence summing between the gamma-rays and X-rays. However, the highest energy X-ray emitted by ${ }^{73} \mathrm{As}$ is $11.103 \mathrm{keV}$. Photons this low in energy have a very low probability of penetrating the aluminum end-cap of the clover detectors, therefore it is assumed there will be no TCS.

${ }^{241} \mathrm{Am}$ decays $\sim 100 \%$ by alpha decay to ${ }^{237} \mathrm{~Np}[32] .{ }^{241} \mathrm{Am}$ also has a small probability of undergoing spontaneous fission [33]. There are several gamma-rays and X-rays associated with ${ }^{241} \mathrm{Am}$ and the ${ }^{237} \mathrm{~Np}$ daughter which could interfere with the calculation of the peak-to-total ratio and should be taken into consideration.

${ }^{109} \mathrm{Cd}$ decays $100 \%$ by electron capture to an isomeric state of ${ }^{109} \mathrm{Ag}$ [34]. This isomeric state has a half-life of 39.6 seconds, so there will not be any TCS between the gamma-ray and Xrays emitted by the decaying nucleus. However, the energies of the X-rays that are emitted by ${ }^{109} \mathrm{Cd}$ range from 22 to $25.5 \mathrm{keV}$. Photons of these energies are energetic enough to penetrate the aluminum covering of the detector. This is especially true because the detector is an n-type detector and the aluminum cover is thin. With p-type detectors and 1-mm aluminum covers, photons this low would not contribute much to the observed spectrum. For this system, the Xrays must be taken into consideration when calculating the peak-to-total ratio for $88 \mathrm{keV}$ gamma-ray from ${ }^{109} \mathrm{Cd}$. 
${ }^{57} \mathrm{Co}$ decays $100 \%$ by electron capture to ${ }^{57} \mathrm{Fe}$ [35]. The X-rays have a maximum energy of $7 \mathrm{keV}$, so they will not penetrate the aluminum end-cap. However, there is a secondary peak at $136 \mathrm{keV}$ that adds a significant number of counts to the total spectrum of ${ }^{57} \mathrm{Co}$. This gammaray is not in coincidence with the main $122 \mathrm{keV}$ photon, but should be accounted for in the peak-to-total ratio calculation.

${ }^{113} \mathrm{Sn}$ decays $100 \%$ by electron capture to ${ }^{113} \mathrm{In}$ [36]. There are two main gammas emitted by ${ }^{113} \mathrm{Sn}$, which have energies of $255 \mathrm{keV}$ and $391 \mathrm{keV}$. It is a cascading emission, beginning with the $255 \mathrm{keV}$ gamma-ray, but the $391 \mathrm{keV}$ gamma-ray originates from an isomeric energy level of the daughter ${ }^{113}$ In, with a half-life of 1.66 hours. The main photon being observed is the $391 \mathrm{keV}$ gamma-ray, so there is no need to account for TCS. However, the Xrays - which can be as energetic as $28 \mathrm{keV}$ - and the $255 \mathrm{keV}$ gamma must be accounted for when calculating the total number of counts in the spectrum due to the $391 \mathrm{keV}$ photon.

${ }^{85} \mathrm{Sr}$ decays $100 \%$ by electron capture to ${ }^{85} \mathrm{Rb}$ [37]. The X-rays have a maximum energy of $15.2 \mathrm{keV}$, which are not energetic enough to significantly penetrate the aluminum end-cap of the clover detectors. ${ }^{85} \mathrm{Sr}$ has no other significant gamma-rays, however, most samples containing ${ }^{85} \mathrm{Sr}$ will also contain ${ }^{82} \mathrm{Sr}$. ${ }^{82} \mathrm{Sr}$ decays to ${ }^{82} \mathrm{Rb}$, which then decays by $\beta^{+}$emission [38]. The half-life of ${ }^{82} \mathrm{Rb}$ much is shorter than the half-life of ${ }^{82} \mathrm{Sr}$ and will reach secular equilibrium within 15 minutes. The $511 \mathrm{keV}$ annihilation photon can interfere with the $514 \mathrm{keV}$ gamma-ray of ${ }^{85} \mathrm{Sr}$ and therefore is cause for concern. The half-life of ${ }^{82} \mathrm{Sr}$ is 25.6 days, while the half-life of ${ }^{85} \mathrm{Sr}$ is 64.84 days. The sample used in this experiment was aged enough to eliminate all detectable traces of ${ }^{82} \mathrm{Sr}$ and retain an appreciable amount of ${ }^{85} \mathrm{Sr}$.

${ }^{54} \mathrm{Mn}$ decays $\sim 100 \%$ by electron capture to ${ }^{54} \mathrm{Cr}$ [39]. There is also a small (<0.00039\%) probability of decaying by $\beta^{-}$decay to ${ }^{54} \mathrm{Fe}$ [39]. The maximum energy of the $\mathrm{X}$-rays associated with ${ }^{54} \mathrm{Mn}$ is $5.95 \mathrm{keV}$, and therefore will not penetrate the aluminum end-cap of the clover detectors. There are no other gamma-rays associated with this nuclide.

${ }^{65} \mathrm{Zn}$ has two decay modes. It decays via electron capture to $98.6 \%$ of the time, with the remainder decaying by $\beta^{+}$emission. Both of these decay modes go to ${ }^{65} \mathrm{Cu}[40]$. The $511 \mathrm{keV}$ annihilation photons contribute to the total number of events in the spectrum and need to be taken into consideration. The maximum X-ray energy is $9.0 \mathrm{keV}$, and therefore produces a 
negligible effect in the spectrum. The $1115 \mathrm{keV}$ gamma-ray is emitted in the electron capture transition and therefore is not in coincidence with the $\beta^{+}$particle that is sometimes emitted.

To obtain the peak-to-total ratio curve, a Canberra peak-to-total source set was obtained. This set consisted of ${ }^{109} \mathrm{Cd},{ }^{57} \mathrm{Co},{ }^{113} \mathrm{Sn},{ }^{137} \mathrm{Cs},{ }^{54} \mathrm{Mn}$, and ${ }^{65} \mathrm{Zn}$ puck-shaped sources, each having an activity of $1.0 \mu \mathrm{Ci} \pm 20 \%$. Because we are only taking the ratio of the observed fullenergy peak events to the total events in the spectrum, the activity of the source does not affect the results, and therefore the $20 \%$ uncertainty in the activity does not play a role in the uncertainty of the results. Because of high energy X-rays, the ${ }^{109} \mathrm{Cd}$ and ${ }^{113} \mathrm{Sn}$ sources had X-ray absorption foils incorporated into them. Later, an ${ }^{241} \mathrm{Am}$ IAEA disk source was incorporated into the data set to obtain a point in the lower-energy range of the spectrum. This was done because the detectors being analyzed are n-type detectors, which are more capable of detecting low-energy photons. Because of this, the expected low-energy hump in the peak-to-total ratio curve is lower for n-type detectors as opposed to p-type detectors. Other sources used later included "massless" sources which consisted of a liquid drop sandwiched between two thin sheets of Mylar. This was done to reduce any self-attenuation that can occur in the sample. These massless sources included ${ }^{73} \mathrm{As},{ }^{109} \mathrm{Cd},{ }^{113} \mathrm{Sn}$, and ${ }^{85} \mathrm{Sr}$. The general equation used to fit the peak-to-total data points is split into two energy regions. In other work [41], the higher energy portion was said to be linear when the logarithm of the energy was plotted against the logarithm of the peak-to-total ratio. The linear portion of the graph for this system was observed to be above $400 \mathrm{keV}$. Below $400 \mathrm{keV}$, a polynomial function is usually fit for the data. The peak-to-total ratio equation determination in Genie 2000 allows the user to choose the degree of polynomial that best fits the low-energy portion of the spectrum [42]. Several polynomial functions were fit to the data, and the one that gave the best fit to the low-energy data was a $4^{\text {th }}$ order polynomial. Therefore, the peak-to-total ratio equation had the following form: 


$$
\begin{gathered}
\ln \left(\frac{P}{T}\right)=a_{0}+a_{1} \ln (E)+a_{2} \ln (E)^{2}+a_{3} \ln (E)^{3}+a_{4} \ln (E)^{4} ; \quad E<400 \mathrm{keV} \\
\ln \left(\frac{P}{T}\right)=a_{5}+a_{6} \ln (E) ; \quad E \geq 400 \mathrm{keV}
\end{gathered}
$$

where:

$P / T$ is the peak-to-total ratio

$E$ is the energy of the photon in keV

$a_{i}$ is the $i^{\text {th }}$ coefficient to be found in the equation

It is known that for a given detector system, the peak-to-total ratio is generally linear above 400 $\mathrm{keV}$ and well past $3000 \mathrm{keV}[30]$, which is currently the upper limit of this detector system. $4^{\text {th }}$ order equation describes the lower energies well, up to about $400 \mathrm{keV}$.

In the first run of experiments, only the six Canberra "puck" sources were used. Prior research has indicated that the peak-to-total ratios should be obtained in an environment free from scattering materials, such as shields or other materials surrounding the detector [43]. However, for the purposes of this system, the Nal(TI) shield will always be in place and will contribute to backscatter events that could contribute to TCS. For this reason, all measurements were taken inside the $\mathrm{Nal}(\mathrm{TI})$ shield in the normal configuration for the system. The data points for Add Back mode using the sources from the Canberra source set can be seen in Figure 18. The $\mathrm{P} / \mathrm{T}$ ratio equation is expected to show a maximum at around $60-100 \mathrm{keV}$ for a p-type detector and around 30-80 keV for an n-type detector. The presence of the aluminum end-cap may vary these values. Because the crystals are n-type germanium detectors, the maximum is expected to occur below $88 \mathrm{keV}$, which is emitted by ${ }^{109} \mathrm{Cd}$. Figure 18 shows the proof of this statement and does not adequately show the expected "hump" shape, so it was determined that a lower-energy point was needed to get the proper fit equation. A disk source of ${ }^{241} \mathrm{Am}(59.5 \mathrm{keV})$ and a massless source of ${ }^{73} \mathrm{As}(53.4 \mathrm{keV})$ were then incorporated into the source set. A massless source of ${ }^{85} \mathrm{Sr}(514.01 \mathrm{keV})$ was also obtained to incorporate more data points for the peak-to-total ratios. 


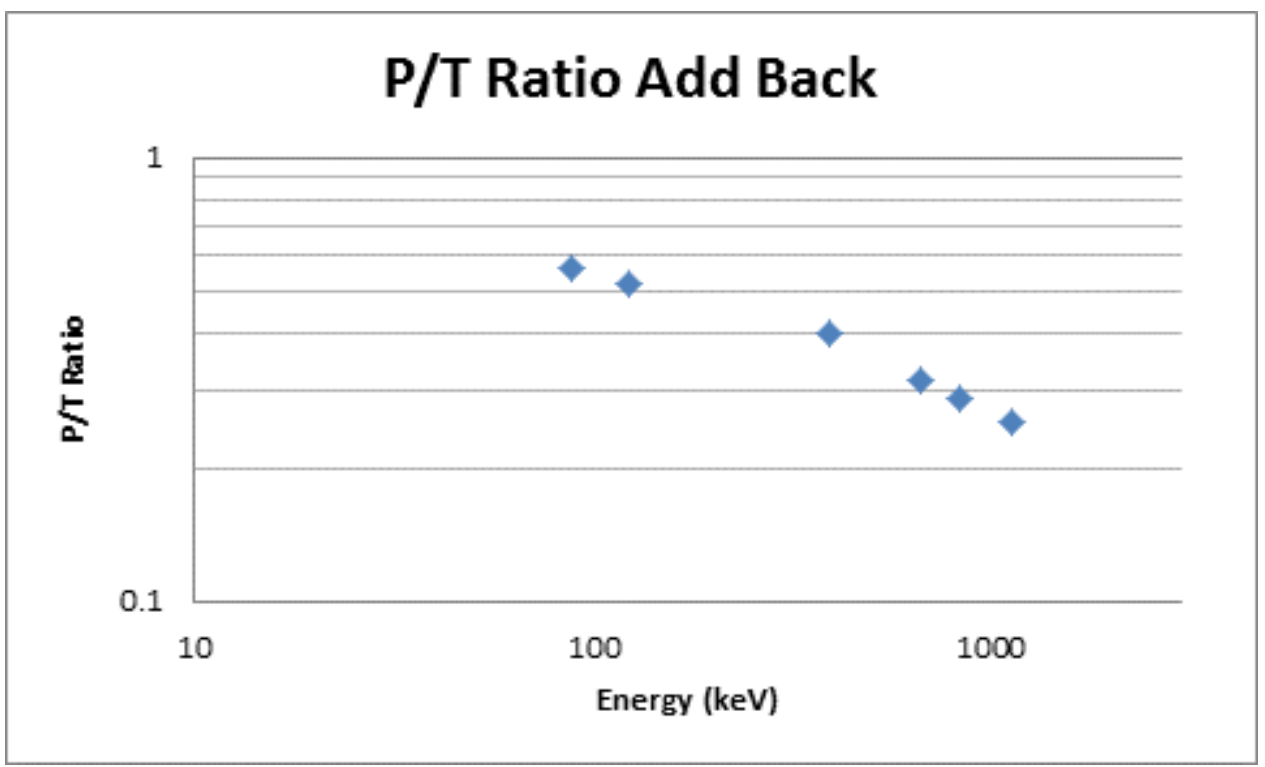

Figure 18: Initial results from peak-to-total ratio counts

Upon further examination of the data, it was seen that the ${ }^{109} \mathrm{Cd}$ and ${ }^{113} \mathrm{Sn}$ sources gave different $\mathrm{P} / \mathrm{T}$ ratios depending on which side of the source was exposed to the detector. This was observed by counting these sources on a completely separate analog detector system consisting of an n-type HPGe detector and putting a 1-mm aluminum shield over the source to mimic the clover detector setup. The ${ }^{109} \mathrm{Cd}$ and ${ }^{113} \mathrm{Sn}$ sources were counted for 30 minutes each and were flipped $180^{\circ}$ and counted for another 30 minutes. The comparison of these spectra can be seen in Figure 19 and Figure 20. 


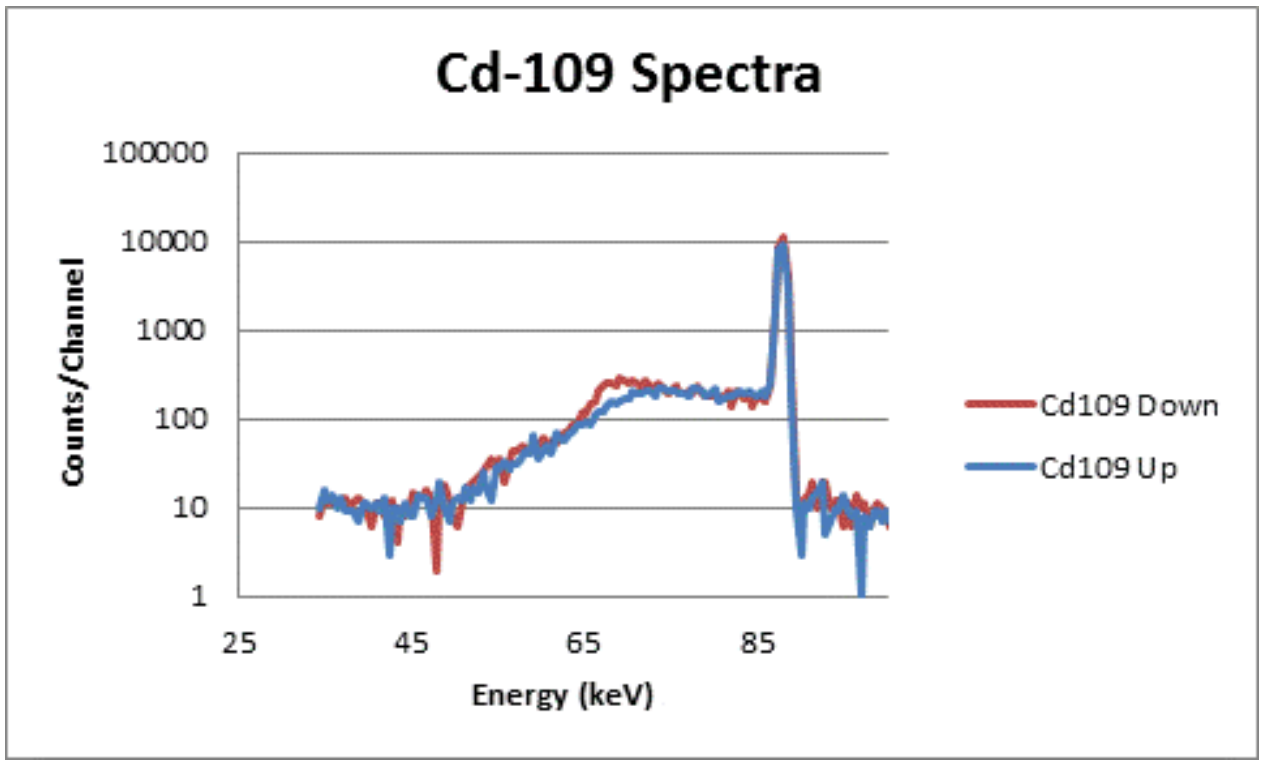

Figure 19: The difference in spectra in turning the $\mathrm{Cd}-109$ source upside-down.

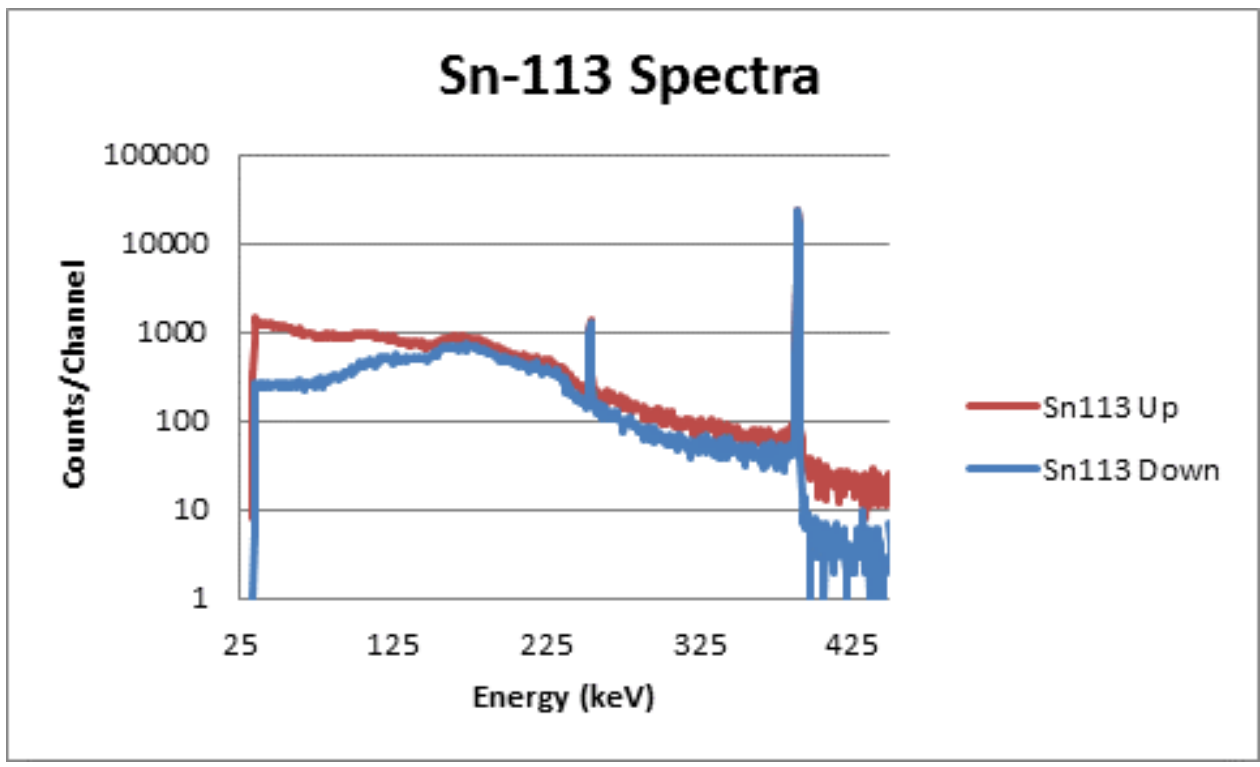

Figure 20: The difference in spectra in turning the Sn-113 source upside-down.

It is obvious that the spectra shown by the two Figures will have different peak-to-total ratios. These observations led us to conclude that the X-ray absorption foil was only incorporated onto one side of the source. It was also observed that these absorption foils also influence the Compton continuum, particularly at lower energies. For these reasons, these ${ }^{109} \mathrm{Cd}$ 
and ${ }^{113} \mathrm{Sn}$ "puck" sources were removed from the peak-to-total ratio determinations and other sources were used instead. These other sources were created in the radiochemistry laboratory at LANL and had an activity of about $1 \mu \mathrm{Ci}$ each. The sources were "massless" sources similar to the ${ }^{85} \mathrm{Sr}$ and ${ }^{73} \mathrm{As}$ sources used in this work. Corrections for the observed X-rays to obtain true peak-to-total ratios will be explained later.

It was expected that the ${ }^{241} \mathrm{Am}$ and ${ }^{73} \mathrm{As}$ sources would give similar peak-to-total ratios because the predominant gamma energies of these two nuclides are very similar. However, the ${ }^{241} \mathrm{Am}$ source consistently gave a lower peak-to-total ratio than the ${ }^{73} \mathrm{As}$ source. The low-energy spectrum of ${ }^{241} \mathrm{Am}$ can be seen in Figure 21 . There are several obvious peaks between 15 and 40 keV from both ${ }^{241} \mathrm{Am}$ and ${ }^{237} \mathrm{~Np}$ in this spectrum which are increasing the peak-to-total ratio in the spectrum.

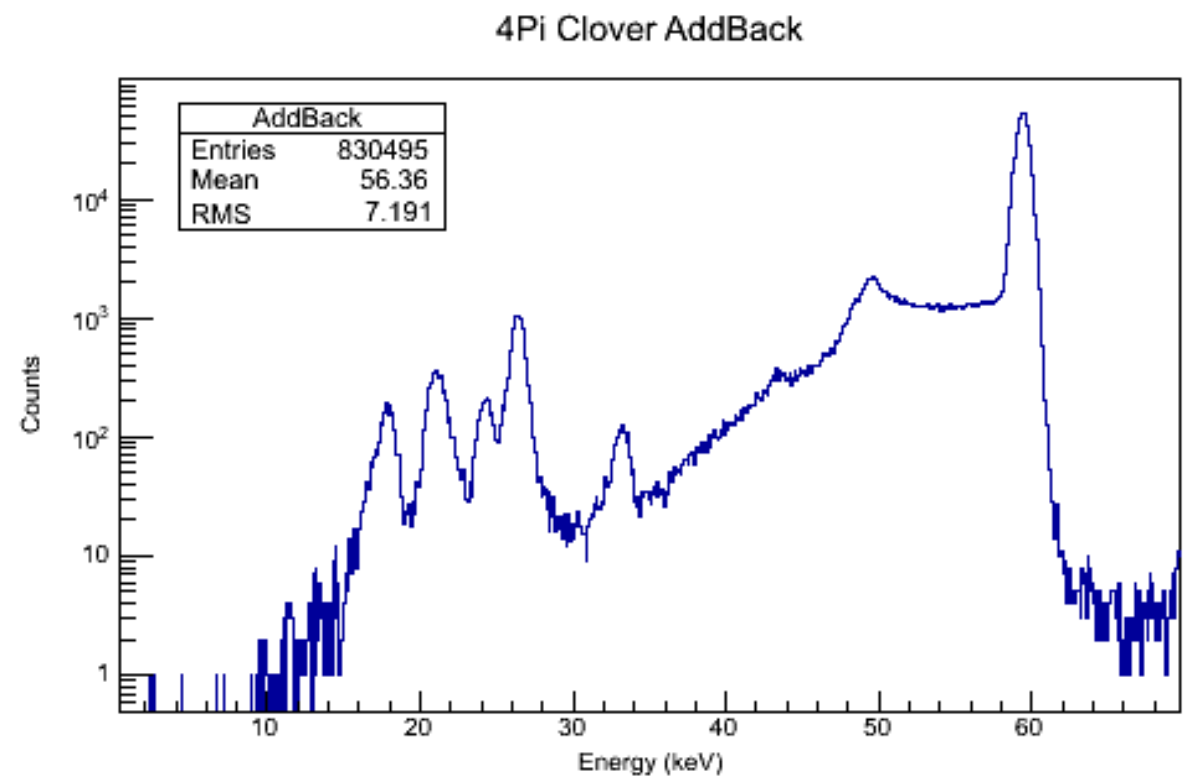

Figure 21: Am-241 spectrum

The spectrum of ${ }^{73} \mathrm{As}$ can be seen in Figure 22. While there are two minor peaks between 20 and $30 \mathrm{keV}$, the spectrum is much cleaner as a whole and much more conducive to obtaining an accurate peak-to-total ratio in this lower-energy region. It was determined that the ${ }^{73}$ As spectrum much more accurately represented the peak-to-total ratio in this energy region than the ${ }^{241} \mathrm{Am}$, and for this reason, the ${ }^{241} \mathrm{Am}$ source was completely removed from the 
analysis. The ${ }^{73}$ As source also more accurately represented the geometry of the samples that would be analyzed on this system. Ultimately there were eight sources used in the peak-to-total ratio analysis: ${ }^{73} \mathrm{As},{ }^{109} \mathrm{Cd},{ }^{113} \mathrm{Sn}$, and ${ }^{85} \mathrm{Sr}$ "massless" sources obtained from the LANL radiochemistry laboratory as well as ${ }^{57} \mathrm{Co},{ }^{137} \mathrm{Cs},{ }^{54} \mathrm{Mn}$, and ${ }^{65} \mathrm{Zn}$ "puck" sources from the Canberra peak-to-total source set.

4Pi Clover AddBack

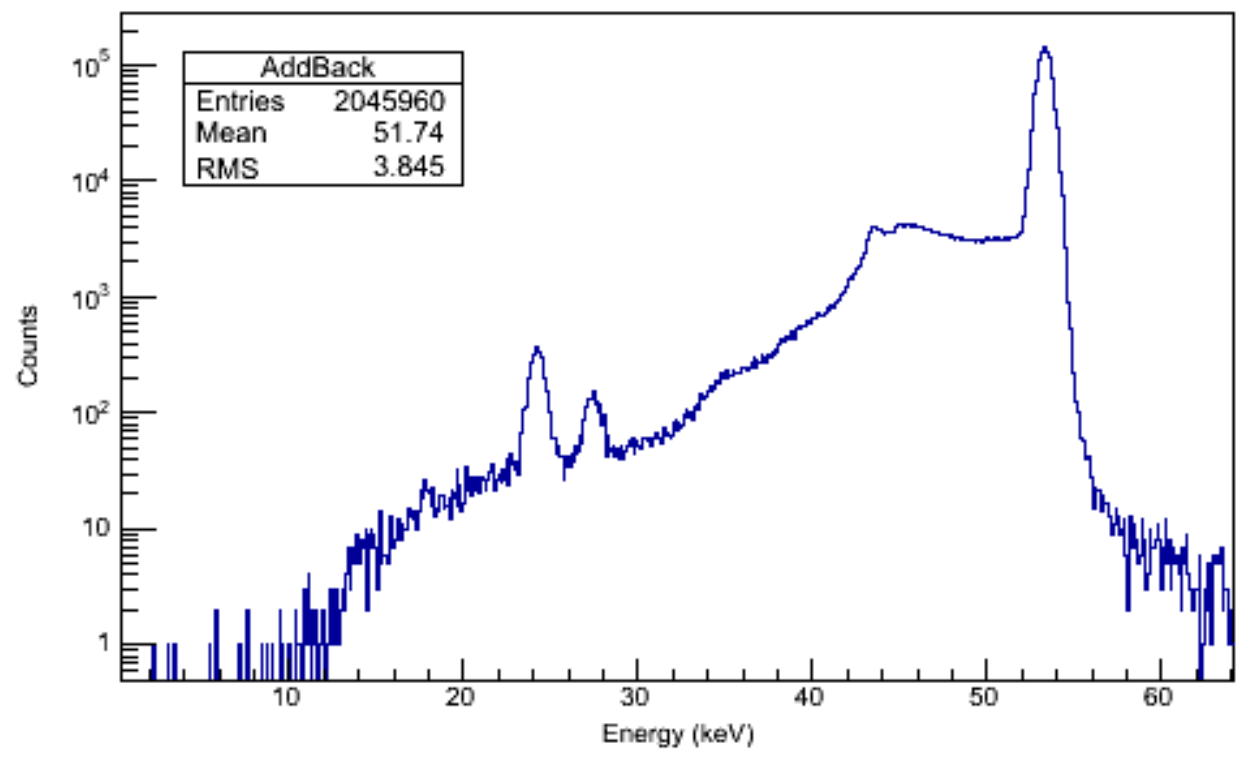

Figure 22: As-73 spectrum

\subsection{Peak-to-Total Ratio Equation Procedure}

To calculate the influence of true-coincidence summing, accurate peak-to-total ratios must be obtained across the energy spectrum. The end goal is to obtain the ratio of the number of counts observed in the peak divided by the total number of counts that can be attributed to that nuclide. This ratio is a function of the energy of the photon, which stems from the fact that cross-sections of different interactions are also functions of photon energy. When this is determined, true-coincidence summing factors can be readily obtained for a given nuclide. The interactions that contribute to the total counts in a spectrum include the photoelectric effect, Compton scattering, and pair production (which includes annihilation photons and escape peaks).

There are several factors that should be taken into consideration when calculating the peak-to-total ratios [41]. First, the peaks themselves should be fit properly taking the 
background continuum into account. This was performed by fitting the observed peaks to the Sampo equation [44]. Uncertainties for the peak fit were calculated and recorded. The total should include all counts in the spectrum from the high-energy side of the peak down to the low-energy threshold as well as any events that can occur below the low-energy threshold. The observed total in the spectrum needs to be corrected for several things: 1 ) the background of the counting environment should be considered, as this will impose additional counts in the spectrum; 2) in all electronic counting systems, low-energy noise from the preamplifier is accounted for by imposing a low-energy cutoff, below which no counts can be recorded. This is inevitable in all spectra, but should be taken into account because low-energy events can still sum with full-energy depositions of coincident photons. This correction depends on which counting mode the peak-to-total ratios are being determined for; 3 ) some of the nuclides being used in the peak-to-total determination are not single gamma emitters, and thus the total observed counts will be inflated from side peaks in the spectrum. Correcting for these peaks is essential to obtaining accurate peak-to-total ratios. Low-energy $X$-rays should also be taken into consideration when calculating the peak-to-total ratio.

\subsubsection{Background Subtraction}

To accurately determine the NORM and cosmic contribution to the spectrum, a good background spectrum must be obtained [41]. For this research, a 14-hour background count was performed with no sample in the system. To estimate the total background contribution to the sample spectra, the count rate of the background spectrum was calculated using the proper energy range for the given sample. The total expected number of counts from background are then calculated and subtracted from the total counts observed in the spectrum. This is done because the observed total counts in the spectrum are inflated by background counts that should not be included in the peak-to-total ratio. This is not necessary when observing peaks in regular spectra because the peaks are produced on top of the continuum, whether that continuum is produced by background radiation or other contributions. The net peak area will not change due to the number of counts in the underlying continuum. 


\subsubsection{Low-Energy Threshold Cutoff Correction}

Noise from the preamplifier and other electronics can influence the low-energy part of a gamma spectrum [41]. Because of this, it is necessary to impose a low-energy threshold below which no pulses are allowed to contribute (see Section 4.3). However, if an interaction in this low-energy region occurs in the crystal in coincidence with a higher-energy interaction within the same crystal, the energy attributed to each interaction will be summed together. Therefore, it is important to account for interactions that may occur below the threshold but can still sum with other coincident gamma-rays. Another reason to use an extrapolation channel is to reduce contributions to the total from low-energy X-rays. To do these things, an extrapolation channel is chosen in the low-energy part of the spectrum above the threshold [41]. The contents of a specified number of bins are averaged above this extrapolation channel and all channels below the extrapolation channel are set to be equal to this averaged value.

As an example, Figure 23 shows the low energy portion of a spectrum, including the low-energy threshold which occurs around $25-30 \mathrm{keV}$ (notice the $\mathrm{y}$-axis is on a linear scale). The extrapolation channel is chosen above this threshold (at say $35 \mathrm{keV}$ ). A specified number of channels above $35 \mathrm{keV}$ are then averaged together - this value will be about 250 counts per channel for this spectrum. The total will then be the sum of the observed contents of the channels from $35 \mathrm{keV}$ to the upper edge of the peak plus the extrapolated values in the channels from $0 \mathrm{keV}$ to $35 \mathrm{keV}$. If low energy $X$-rays are observed in the spectrum, such as that seen in Figure 24, then the extrapolation channel should be chosen to be above the X-ray energy. This way, the X-ray has no influence on the spectrum (aside from possible minor X-gamma summing and random summing). 


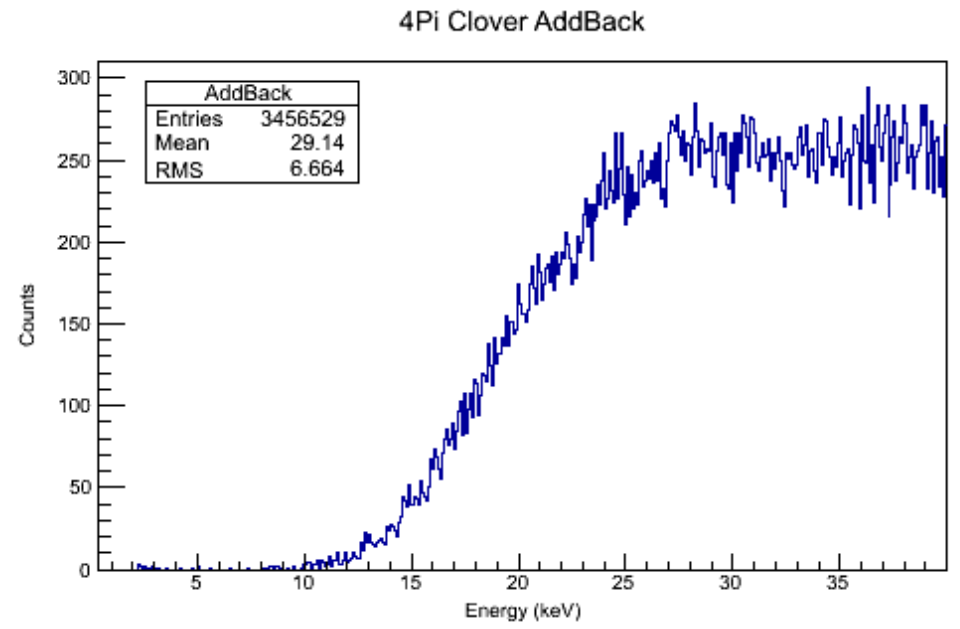

Figure 23: Low energy portion of a typical spectrum

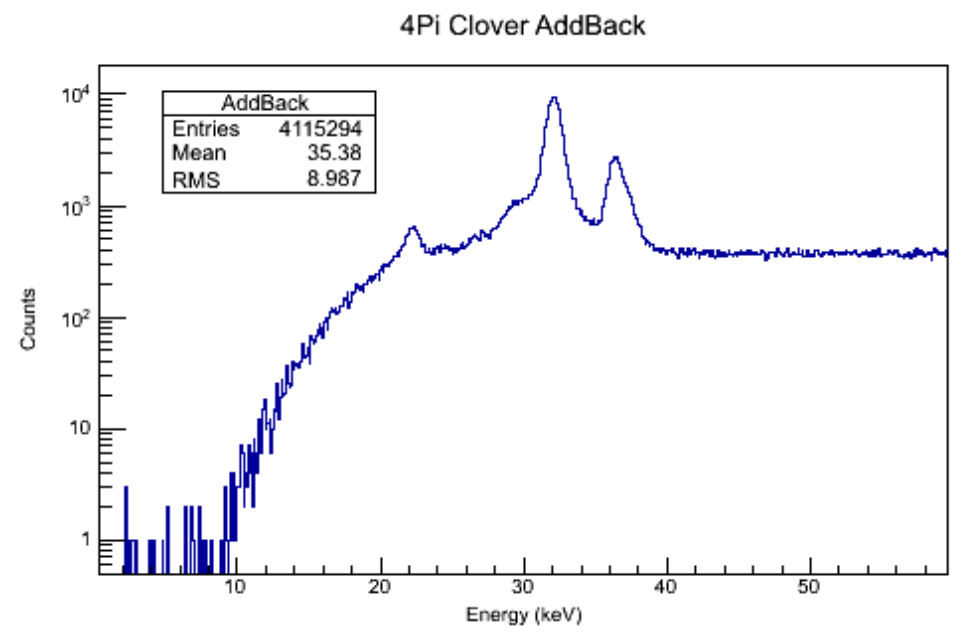

Figure 24: Some samples have X-rays in the low energy portion of their spectra. 
Special consideration needs to be taken for the peak-to-total ratio of Add Back mode. A situation can arise in which a cascading emission occurs and the full energy of one of the photons is deposited in a single crystal. If another photon deposits a small amount of its energy (below the low-energy threshold) into a different crystal and does not interact anywhere else in the system, then Add Back mode will only record the full-energy deposition from the first photon and nothing from the second photon. Therefore, events that would contribute to the spectrum below the threshold will not have an effect on the coinciding gamma-ray. If these two interactions had occurred within the same crystal, then Add Back mode would record the sum of these two interactions in the spectrum. For cascading emissions in which one photon deposits all of its energy into the system and another photon deposits a small (below the threshold) amount of energy into the system, the system will only be affected by TCS roughly $1 / 8$ of the time. This is assuming a given photon is equally likely to interact in all detectors. Alternatively in Singles mode, coincident events are only summed together if they occur in the same detector crystal. For the purposes of this research, the peak-to-total ratio equation for Add Back mode was computed without using the extrapolation channel method for any of the nuclides as it was assumed that the relative number of below-threshold events that would contribute to TCS was negligible. As mentioned previously, only $1 / 8$ of the events that deposit energy below the threshold will contribute to TCS in Add Back mode. For most photons, the number of events that occur below the low-energy threshold are relatively small to begin with; therefore, the omission of the extrapolation channel for Add Back mode is justified for most gamma-ray emissions. The X-ray peaks were fit and subtracted from the total before the spectrum was incorporated into the determination of the peak-to-total ratio equation. The peak-to-total ratio at the X-ray energies is assumed to be close to 1.0 because the photoelectric effect is the dominant interaction type at this energy [4]. Therefore the continuum generated by the X-rays does not need to be taken into account.

\subsubsection{Side Peak Correction}

A few of the nuclides used to calculate the peak-to-total ratio equation have more than one peak that significantly alters the peak-to-total ratio observed in the spectrum. This must be corrected in order to obtain accurate peak-to-total ratio values [41]. The correction for side peaks involves an iterative process [41]. The spectra are analyzed initially for the peak-to-total 
ratios before doing any corrections. These initial points are then fit to Equation (6.1) to give an initial guess for the $\mathrm{P} / \mathrm{T}$ ratio equation:

$$
\begin{gathered}
\ln \left(\frac{P}{T}\right)=b_{0}+b_{1} \ln (E)+b_{2} \ln (E)^{2}+b_{3} \ln (E)^{3}+b_{4} \ln (E)^{4} ; \quad E<400 \mathrm{keV} \\
\ln \left(\frac{P}{T}\right)=b_{5}+b_{6} \ln (E) ; \quad E \geq 400 \mathrm{keV}
\end{gathered}
$$

where $b_{i}$ is an initial guess for $a_{i}$ which represent the coefficients of the true peak-to-total equation. The contributions of the side peaks to the total counts in the spectra are then calculated by isolating $T$ is Equation (6.2) and plugging in the side peak energy:

$$
\begin{gathered}
T(E)=P(E) * e^{-\left(b_{0}+b_{1} \ln (E)+b_{2} \ln (E)^{2}+b_{3} \ln (E)^{3}+b_{4} \ln (E)^{4}\right)} ; \quad E<400 \mathrm{keV} \\
T(E)=P(E) * e^{-\left(b_{5}+b_{6} \ln (E)\right)} ; \quad E \geq 400 \mathrm{keV}
\end{gathered}
$$

The total contribution of the side peaks is then subtracted from the totals in their respective spectra:

$$
T_{\text {new }}=T_{\text {old }}-T_{\text {side }}
$$

where:

$T_{\text {new }}$ is the new value for the total to be used in the next iteration

$T_{\text {old }}$ is the old value of the total from the previous iteration

$T_{\text {side }}$ is the current value of the total for the side peak calculated in Equation (6.3)

The difference between the total in the original spectrum and the total expected contribution of the side peak to the spectrum is then used as the new total for that spectrum. This is done for each spectrum containing side peaks. New P/T ratios are calculated and fit again to Equation (6.1) to produce new $b_{i}$ values for Equation (6.2). This process is repeated until the $\mathrm{P} / \mathrm{T}$ ratios converge and the final $\mathrm{P} / \mathrm{T}$ ratio equation has been found.

\subsubsection{Uncertainty Calculations}

The uncertainty in a quotient can be expressed as [4]: 


$$
\sigma_{f}=f \sqrt{\left(\frac{\sigma_{A}}{A}\right)^{2}+\left(\frac{\sigma_{B}}{B}\right)^{2}}
$$

where:

$f=A / B$

$\sigma_{A}$ is the uncertainty in $A$

$\sigma_{B}$ is the uncertainty in $B$

From Equation (6.5), the uncertainty in the peak-to-total ratio is:

$$
\sigma_{P / T}=P / T \sqrt{\left(\frac{\sigma_{P}}{P}\right)^{2}+\left(\frac{\sigma_{T}}{T}\right)^{2}}
$$

where:

$P$ is the peak area

$T$ is the total counts due to the photon of interest

$\sigma_{P}$ is the uncertainty in the peak area

$\sigma_{T}$ is the uncertainty in the total

The uncertainty in the peak area is obtained from the peak-fitting procedure. The uncertainty for $\sigma_{T}$ depends on the corrections that were used to obtain the total. The total $T$ can be expressed as: 


$$
T=T_{O}+T_{E}-T_{B}-T_{S}
$$

where:

$T_{O}$ is the observed total

$T_{E}$ is the total calculated below the extrapolation point

$T_{B}$ is the total contribution from the background

$T_{S}$ is the total contribution from side peaks

The variance of a sum or difference is given by [4]:

$$
\sigma_{f}^{2}=\sigma_{A}^{2}+\sigma_{B}^{2}
$$

where $f=A \pm B$. All of the contributions in Equation (6.7) stem from radioactive decay processes and follow Poisson statistics [4]. The variance of a term that follows Poisson statistics is equal to the true mean of the term:

$$
\sigma_{i}^{2}=\mu_{i}
$$

The true mean of each term can be estimated by the term itself:

$$
\mu_{i}=i
$$

Using Equations (6.7), (6.8), (6.9), and (6.10), the variance in the total can be estimated by:

$$
\sigma_{\mathrm{T}}^{2}=\mathrm{T}_{\mathrm{O}}+\mathrm{T}_{\mathrm{E}}+\mathrm{T}_{\mathrm{B}}+\mathrm{T}_{\mathrm{S}}
$$

The calculation of the uncertainty for best-fit equations is described in Appendix 1 . The Fisher matrix is used to find the estimate of the covariance matrix, and the uncertainty of the fit estimate is obtained using the covariance matrix [45]:

$$
C_{j k}=\sum_{i=1}^{n} w_{i} \frac{\partial f}{\partial a_{j}} \frac{\partial f}{\partial a_{k}}
$$

where:

$\boldsymbol{C}$ is the Fisher matrix

$w_{i}$ is the inverse of the variance for the observed $\mathrm{P} / \mathrm{T}$ ratio of point $i$

$a_{j}$ is the $j^{\text {th }}$ coefficient of the peak-to-total ratio equation

The covariance matrix $\boldsymbol{V}$ is obtained by inverting the Fisher matrix [45]:

$$
V=C^{-1}
$$

The partial derivatives of the $\mathrm{P} / \mathrm{T}$ ratio equation can be expressed as: 


$$
\frac{\partial f}{\partial a_{j}}=\ln (E)^{j}
$$

where:

$j$ goes from 0 to 4 for low energies $(<400 \mathrm{keV}$ ) and from 0 to 1 for high energies ( $\geq 400 \mathrm{keV}$ )

$E$ is in units of keV

The covariance matrix $\boldsymbol{V}$ for the $\mathrm{P} / \mathrm{T}$ ratio equation in Add Back mode is given for reference:

$$
\begin{gathered}
V=\left[\begin{array}{ccccc}
258.4 & -196.7 & 55.28 & -6.797 & 0.3088 \\
-196.7 & 149.9 & -42.16 & 5.188 & -0.2359 \\
55.28 & -42.16 & 11.87 & -1.462 & 0.06651 \\
-6.797 & 5.188 & -1.462 & 0.1802 & -0.008203 \\
.3088 & -0.2359 & 0.06651 & -0.008203 & 0.0003738
\end{array}\right] \quad E<400 \mathrm{keV} \\
V=\left[\begin{array}{ccc}
0.03317 & -0.005096 \\
-0.005096 & 0.0007854
\end{array}\right] \quad E \geq 400 \mathrm{keV}
\end{gathered}
$$

\subsubsection{Results}

The peak-to-total ratios have been found for several modes of operation: Add Back, Singles, Add Back Misty, Add Back Kerri, and each HPGe crystal individually. The full data and final equations can be found in Appendix 2. The general form of the $P / T$ ratio equation is given by Equation (6.1), and the coefficients for Add Back and Singles mode are given in Table 2 for reference. The Add Back and Singles mode graphs are shown in Figure 25.

Table 2: Coefficients for P/T ratio equations for Add Back and Singles modes.

\begin{tabular}{|c|c|c|}
\hline Coefficient & Add Back Value & Singles Value \\
\hline$a_{0}$ & -2.787 & -5.742 \\
\hline$a_{1}$ & 1.232 & 2.494 \\
\hline$a_{2}$ & -0.0678 & -0.1166 \\
\hline$a_{3}$ & -0.03205 & -0.06486 \\
\hline$a_{4}$ & 0.00299 & .00592 \\
\hline$a_{5}$ & 1.594 & 3.061 \\
\hline$a_{6}$ & -0.4140 & -0.7291 \\
\hline
\end{tabular}

The impact of using Add Back mode is quite clear when compared to Singles mode. The slope of the high-energy line is significantly steeper for Singles mode, which is explained by the increased number of full-energy events added into the peak in Add Back mode. In Singles mode, 
these events only further contribute to the Compton continuum. This is especially noticeable at higher energies up to $3000 \mathrm{keV}$ where the Compton scattering interaction cross-section dominates [4]. At low energies, the peak-to-total ratio is higher in Add Back mode because events below the low-energy threshold are not taken into account due to the nature of TCS in Add Back mode (see Section 7.2).

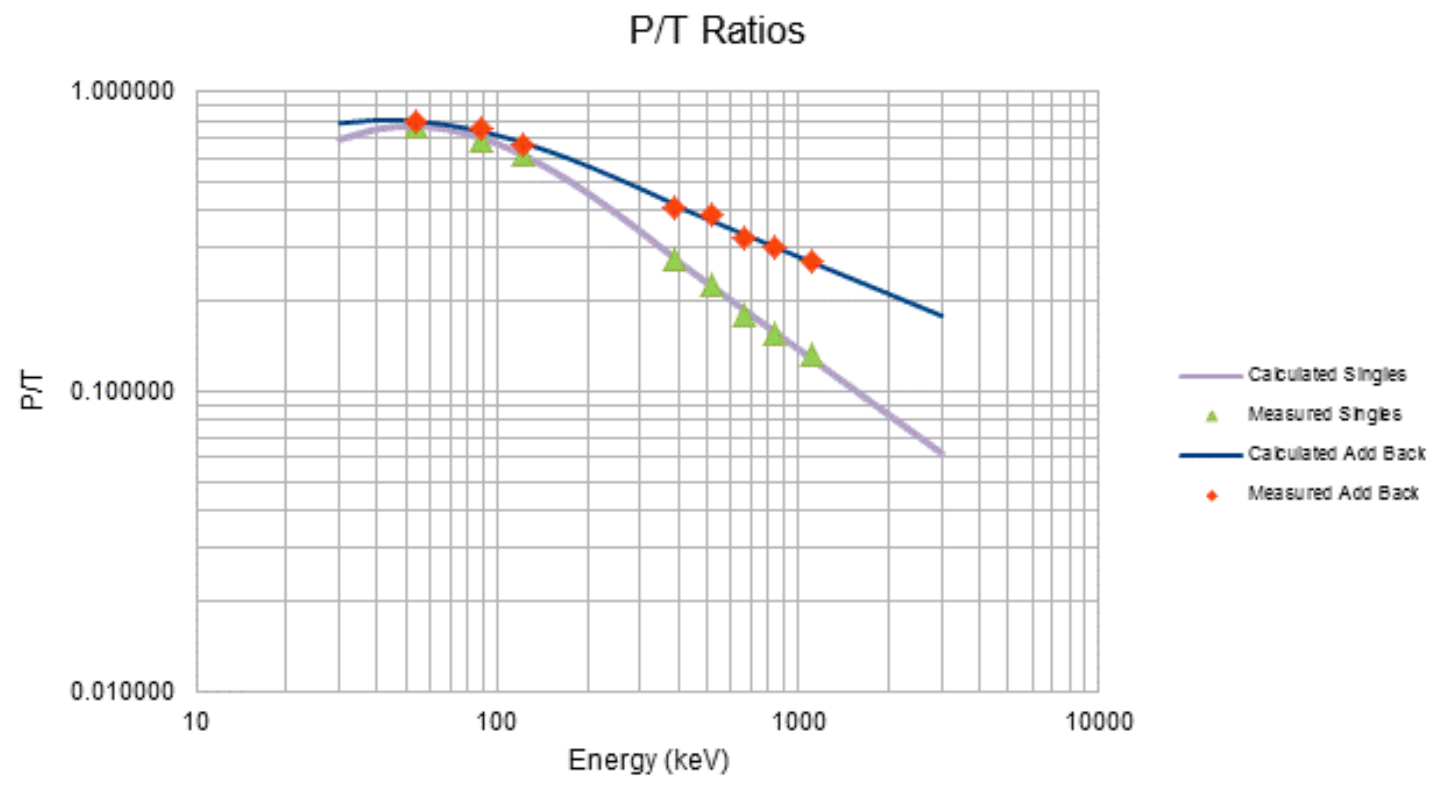

Figure 25: Peak-to-total ratio equation for Singles and Add Back modes 


\section{Chapter 7: Efficiency Curves}

To properly analyze radioactive samples for radionuclide activity levels, accurate efficiency curves must exist for the detector system in its various counting modes. The peak efficiency of a detector system for a given photon energy is defined as the number of recorded full-energy peak events divided by the number of photons emitted during the counting live time [4]. It represents the probability that an emitted photon will be detected by the system, and that the energy will be recorded in full. This peak efficiency will depend on a number of factors including the detector size, counting geometry, and sample shape. The efficiency curves for this system have been determined for two sample geometries. The first geometry is a point source, termed a Type 1 source by LANL convention. The second is a disk source, termed a Type 3 source by LANL convention. These sources are each composed of multiple radionuclides that emit gamma-rays with energies from $60 \mathrm{keV}$ to $1836 \mathrm{keV}$. These sources were counted so that each peak being analyzed in the spectrum had at least 10000 counts in both Add Back mode and Singles mode. Specifically, 10000 counts were chosen because the peak shape in the spectrum is assumed to follow a Poisson distribution [4]. For Poisson distributions, the variance in the data is assumed to be equal to the mean of the data. Because the standard deviation is the square root of the variance, and the true mean is estimated by the observed value in the spectrum, peak areas greater than 10000 counts will have a standard deviation less than $1 \%$.

Both of the sources are composed of: ${ }^{241} \mathrm{Am},{ }^{109} \mathrm{Cd},{ }^{57} \mathrm{Co},{ }^{139} \mathrm{Ce},{ }^{203} \mathrm{Hg},{ }^{113} \mathrm{Sn},{ }^{137} \mathrm{Cs},{ }^{88} \mathrm{Y}$, and

${ }^{60} \mathrm{Co}$. It should be noted that each of these radionuclides emit their main gamma-rays mainly as singlets except ${ }^{88} \mathrm{Y}$ and ${ }^{60} \mathrm{Co} .{ }^{88} \mathrm{Y}$ and ${ }^{60} \mathrm{Co}$ both have two main gamma-rays that are emitted in coincidence with each other close to $100 \%$ of the time, making these photons very susceptible to true-coincidence summing. ${ }^{139} \mathrm{Ce}$ is also susceptible to gamma-X coincidence summing. The correction for these will be explained later.

\subsection{Methods}

The two sources used for the efficiency curve determination were supplied with data sheets, outlining the gamma energies, activities, and half-lives for all gamma-emitting nuclides in the source. The pertinent data for the Type 3 source is given in Table 3. For a Type 1 point source, the data is provided in Appendix 3. The data sheets for the multi-line gamma source are provided in Appendix 5. 
Table 3: Type 3 multi-line gamma source information

\begin{tabular}{|c|c|c|c|}
\hline Nuclide & Energy (keV) & $\begin{array}{c}\text { Activity at Creation } \\
\text { ( } \nu \mathrm{ps})\end{array}$ & Half-Life (days) \\
\hline${ }^{241} \mathrm{Am}$ & 59.5 & $332 \pm 6$ & $1.580 \mathrm{E}+05$ \\
\hline${ }^{109} \mathrm{Cd}$ & 88.0 & $482 \pm 17$ & $4.626 \mathrm{E}+02$ \\
\hline${ }^{57} \mathrm{Co}$ & 122.1 & $260 \pm 8$ & $2.718 \mathrm{E}+02$ \\
\hline${ }^{139} \mathrm{Ce}$ & 165.9 & $363 \pm 9$ & $1.376 \mathrm{E}+02$ \\
\hline${ }^{203} \mathrm{Hg}$ & 279.2 & $843 \pm 21$ & $4.661 \mathrm{E}+01$ \\
\hline${ }^{113} \mathrm{Sn}$ & 391.7 & $506 \pm 13$ & $1.151 \mathrm{E}+02$ \\
\hline${ }^{137} \mathrm{Cs}$ & 661.7 & $322 \pm 9$ & $1.098 \mathrm{E}+04$ \\
\hline${ }^{88} \mathrm{Y}$ & 898.0 & $1234 \pm 31$ & $1.066 \mathrm{E}+02$ \\
\hline${ }^{60} \mathrm{Co}$ & 1173.2 & $616 \pm 16$ & $1.925 \mathrm{E}+03$ \\
\hline${ }^{60} \mathrm{Co}$ & 1332.5 & $616 \pm 16$ & $1.925 \mathrm{E}+03$ \\
\hline${ }^{88} \mathrm{Y}$ & 1836.1 & $1307 \pm 34$ & $1.066 \mathrm{E}+02$ \\
\hline
\end{tabular}

The first step in determining the efficiencies is to correct the activity for each nuclide to the activity at count time. The creation date for the Type 3 source is April 1, 2011. The source was counted on July 27,2012 , so there is a difference of 483 days between source creation and counting. The formula for correcting the activity is [4]:

$$
A=A_{0} e^{-\lambda t}
$$

where:

$A$ is the present activity

$A_{o}$ is the activity at the time of creation

$\lambda$ is the decay constant of the nuclide in (days) ${ }^{-1}$

$t$ is the number of days between the creation date and counting date

Using this formula, the activities during counting time were found for each nuclide in the Type3 source and are given in Table 4. 
Table 4: Activity decay correction for Type 3 multi-gamma source

\begin{tabular}{|c|c|c|c|}
\hline Nuclide & $\begin{array}{c}\text { Activity at Creation } \\
(\gamma p s)\end{array}$ & Half-Life (days) & $\begin{array}{c}\text { Activity at Count Time } \\
(\gamma p s)\end{array}$ \\
\hline${ }^{241} \mathrm{Am}$ & $332 \pm 6$ & $1.580 \mathrm{E}+05$ & $331 \pm 6$ \\
\hline${ }^{109} \mathrm{Cd}$ & $482 \pm 17$ & $4.626 \mathrm{E}+02$ & $234 \pm 8$ \\
\hline${ }^{57} \mathrm{Co}$ & $260 \pm 8$ & $2.718 \mathrm{E}+02$ & $75.9 \pm 2.2$ \\
\hline${ }^{139} \mathrm{Ce}$ & $363 \pm 9$ & $1.376 \mathrm{E}+02$ & $31.8 \pm 0.8$ \\
\hline${ }^{203} \mathrm{Hg}$ & $843 \pm 21$ & $4.661 \mathrm{E}+01$ & $0.640 \pm 0.016$ \\
\hline${ }^{113} \mathrm{Sn}$ & $506 \pm 13$ & $1.151 \mathrm{E}+02$ & $27.6 \pm 0.7$ \\
\hline${ }^{137} \mathrm{Cs}$ & $322 \pm 9$ & $1.098 \mathrm{E}+04$ & $312 \pm 9$ \\
\hline${ }^{88} \mathrm{Y}$ & $1234 \pm 31$ & $1.066 \mathrm{E}+02$ & $53.40 \pm 1.33$ \\
\hline${ }^{60} \mathrm{Co}$ & $616 \pm 16$ & $1.925 \mathrm{E}+03$ & $517 \pm 13$ \\
\hline${ }^{60} \mathrm{Co}$ & $616 \pm 16$ & $1.925 \mathrm{E}+03$ & $517 \pm 13$ \\
\hline${ }^{88} \mathrm{Y}$ & $1307 \pm 34$ & $1.066 \mathrm{E}+02$ & $56.55 \pm 1.47$ \\
\hline
\end{tabular}

For the single-gamma emitters, the efficiency can be calculated by simply taking the count rate observed in the detectors (corrected for dead-time) and dividing by the activity at count time, as given by [4]:

$$
\varepsilon(E)=\frac{C R(E)}{A}
$$

where:

$\varepsilon(E)$ is the peak-efficiency for the counting mode at energy $E$

$C R(E)$ is the dead-time corrected count rate observed in the spectrum at energy $E$ $A$ is the activity of the nuclide at the time of counting

To correct the observed count rate for dead-time, the fraction of lost events must be calculated. This correction will be small at low dead-times, but can significantly alter the spectrum if the dead-time is high. This calculation can be done using the methods described in Section 8.3. For the dual-gamma emitters, the process is more complex.

The count rate in Equation (7.2) can be expressed as:

$$
C R(E)=\frac{P(E)}{t}
$$

where:

$P(E)$ is the number of count in the peak at energy $E$

$t$ is the Live Time of the count 
Substituting Equation (7.3) into Equation (7.2), the variance in $\varepsilon(E)$ can be expressed as the variance of a quotient [4]:

$$
\sigma_{\varepsilon}^{2}=\left(\frac{P(E)}{t A}\right)^{2}\left[\left(\frac{\sigma_{P}}{P(E)}\right)^{2}+\left(\frac{\sigma_{A}}{A}\right)^{2}\right]
$$

where $\sigma_{i}$ is the uncertainty in $i$. It is assumed that the uncertainty in the Live Time $t$ is negligible compared to the uncertainty of the other factors.

\subsection{True-Coincidence Summing}

A gamma-ray emission that suffers from true-coincidence summing produces a peak that has a reduced area, due to summing with other photons. In order to account for truecoincidence summing, the probability of a full-energy deposition of the photon of interest coinciding with an event attributable to another photon must be calculated. This can be expressed by the following equation [13]:

$$
F=\varepsilon_{T, C} p
$$

where:

$F$ is the coincidence summing factor

$\varepsilon_{T, C}$ is the total efficiency of the coinciding photon

$p$ is the probability that the coinciding photon was emitted given that the main gamma was emitted

Equation (7.5) can be rewritten in terms of the peak efficiency and the peak-to-total ratio:

$$
F=\frac{\varepsilon_{P, C}}{P / T} p
$$

because $\varepsilon_{P}=\varepsilon_{T}^{*}(P / T)$. Now if $F$ is multiplied on the top and bottom by $\varepsilon_{P, M}$ (the peak efficiency of the main gamma-ray), Equation (7.6) becomes:

$$
F=\frac{\varepsilon_{P, M} \varepsilon_{P, C}}{\varepsilon_{P, M} * P / T} p
$$

and this can be simplified to:

$$
F=\frac{S}{\varepsilon_{P, M} * P / T}
$$

where $S$ is the probability of detecting the full-energy deposition from both photons in the same detector in the same time window. This will result in a contribution to the sum peak: 


$$
S=\varepsilon_{P, M} \varepsilon_{P, C} p
$$

This can be calculated by finding the number of coincident pairs that have been emitted from the sample and observing the number of counts in the sum peak of the spectrum:

$$
S=\frac{C_{S}}{t * A_{S}}
$$

where:

$C_{S}$ is the area of the sum peak

$t$ is the count time

$A_{S}$ is the gamma-ray emission rate of the coincident pairs in the sample

To correct the observable peak suffering from true-coincidence summing, the following equation can be used:

$$
a_{C}=\frac{a_{O}}{1-F}
$$

where:

$a_{C}$ is the corrected peak area

$a_{O}$ is the observed peak area

$F$ is the coincidence summing factor

The full-energy peak efficiency $\varepsilon_{p}$ then becomes:

$$
\begin{aligned}
\varepsilon_{P}(E) & =\frac{a_{c}}{t A} \\
& =\frac{a_{O}}{t A(1-F)} \\
& =\frac{a_{O}}{t A\left(1-\frac{S}{\varepsilon_{P} * P / T}\right)}
\end{aligned}
$$

where $t$ is the count length in seconds. In this situation, $\varepsilon_{P}$ is seen to be a function of itself. This can be solved transcendentally, graphically, or using an iterative process. Equation (7.12) can be rewritten: 


$$
\begin{gathered}
\varepsilon_{i}=\frac{a_{O}}{t A\left(1-\frac{S}{\varepsilon_{i-1} * P / T}\right)} \\
\varepsilon_{0}=\frac{a_{0}}{t A}
\end{gathered}
$$

where:

$\varepsilon_{0}$ is the original guess of the peak efficiency

$a_{0}$ is the uncorrected peak area of the photon

$t$ is the count length

$A$ is the gamma-emission rate of the photon

By substituting Equation (7.14) into Equation (7.13), $\varepsilon_{i}$ is further simplified to:

$$
\varepsilon_{i}=\frac{\varepsilon_{O}}{1-\frac{S}{\varepsilon_{i-1} * P / T}}
$$

Writing out the first few terms of Equation (7.15), the following sequence is obtained:

$$
\begin{aligned}
& \varepsilon_{1}=\frac{\varepsilon_{O}}{1-\frac{S}{\varepsilon_{0} * P / T}}=\frac{\varepsilon_{0}{ }^{2}}{\varepsilon_{0}-\frac{S}{P / T}} \\
& \varepsilon_{2}=\frac{\varepsilon_{O}}{1-\frac{S}{\varepsilon_{1}{ }^{*} P / T}}=\frac{\varepsilon_{0}{ }^{3}}{\varepsilon_{0}^{2}-\varepsilon_{0} \frac{S}{P / T}+\left(\frac{S}{P / T}\right)^{2}} \\
& \varepsilon_{3}=\frac{\varepsilon_{0}}{1-\frac{S}{\varepsilon_{2} * P / T}}=\frac{\varepsilon_{0}{ }^{4}}{\varepsilon_{0}{ }^{3}-\varepsilon_{0}{ }^{2} \frac{S}{P / T}+\varepsilon_{0}\left(\frac{S}{P / T}\right)^{2}-\left(\frac{S}{P / T}\right)^{3}} \\
& \varepsilon_{i}=\frac{\varepsilon_{O}}{1-\frac{S}{\varepsilon_{i-1} * P / T}} \\
& =\frac{\varepsilon_{0}^{i+1}}{\varepsilon_{0}{ }^{i}-\varepsilon_{0}{ }^{i-1}\left(\frac{S}{P / T}\right)^{1}+\ldots+(-1)^{i-1} \varepsilon_{0}{ }^{1}\left(\frac{S}{P / T}\right)^{i-1}+(-1)^{i}\left(\frac{S}{P / T}\right)^{i}}
\end{aligned}
$$


The denominator of Equation (7.19) is a converging series, the sum of which can be obtained as follows:

$$
\begin{aligned}
& \Sigma=\varepsilon_{0}^{i}-\varepsilon_{0}^{i-1}\left(\frac{S}{P / T}\right)^{1}+\ldots+(-1)^{i-1} \varepsilon_{0}{ }^{1}\left(\frac{S}{P / T}\right)^{i-1}+(-1)^{i}\left(\frac{S}{P / T}\right)^{i} \\
& \Sigma *\left(\varepsilon_{0} \frac{P / T}{S}\right)=\left(\varepsilon_{0} \frac{P / T}{S}\right)\left[\varepsilon_{0}{ }^{i}-\varepsilon_{0}{ }^{i-1}\left(\frac{S}{P / T}\right)^{1}+\ldots+(-1)^{i-1} \varepsilon_{0}{ }^{1}\left(\frac{S}{P / T}\right)^{i-1}\right. \\
& \left.+(-1)^{i}\left(\frac{S}{P / T}\right)^{i}\right] \\
& \Sigma *\left(\varepsilon_{0} \frac{P / T}{S}\right)=\varepsilon_{0}^{i+1}\left(\frac{S}{P / T}\right)^{-1}-\varepsilon_{0}^{i}+\ldots+(-1)^{i-1} \varepsilon_{0}^{2}\left(\frac{S}{P / T}\right)^{i-2} \\
& +(-1)^{i} \varepsilon_{0}\left(\frac{S}{P / T}\right)^{i-1} \\
& \Sigma+\Sigma *\left(\varepsilon_{0} \frac{P / T}{S}\right)=\Sigma *\left(1+\varepsilon_{0} \frac{P / T}{S}\right) \\
& =\varepsilon_{0}{ }^{i+1}\left(\frac{S}{P / T}\right)^{-1}+(-1)^{i} \varepsilon_{0}\left(\frac{S}{P / T}\right)^{i-1} \\
& \Sigma=\frac{\varepsilon_{0}^{i+1}\left(\frac{S}{P / T}\right)^{-1}+(-1)^{i} \varepsilon_{0}\left(\frac{S}{P / T}\right)^{i-1}}{1+\varepsilon_{0} \frac{P / T}{S}} \\
& =\frac{\varepsilon_{0}^{i+1}+(-1)^{i} \varepsilon_{0}\left(\frac{S}{P / T}\right)^{i}}{\frac{S}{P / T}+\varepsilon_{0}}
\end{aligned}
$$

Plugging Equation (7.24) into the denominator of Equation (7.19): 


$$
\begin{gathered}
\varepsilon_{i}=\frac{\varepsilon_{0}^{i+1}}{\left.\frac{\varepsilon_{0}^{i+1}+(-1)^{i} \varepsilon_{0}\left(\frac{S}{P / T}\right)^{i}}{\frac{S}{P / T}+\varepsilon_{0}}\right)} \\
\varepsilon_{i}=\frac{\varepsilon_{0}^{i+1}\left(\frac{S}{P / T}+\varepsilon_{0}\right)}{\varepsilon_{0}^{i+1}+(-1)^{i} \varepsilon_{0}\left(\frac{S}{P / T}\right)^{i}} \\
\varepsilon_{i}=\frac{\left.\varepsilon_{0}{ }^{i\left(\frac{S}{P} / T\right.}+\varepsilon_{0}\right)}{\varepsilon_{0}^{i}+(-1)^{i}\left(\frac{S}{P / T}\right)^{i}}
\end{gathered}
$$

Assuming $S /(P / T)$ is less than $\varepsilon_{0}$, if $i$ goes to infinity, Equation (7.27) can be reduced to:

$$
\varepsilon=\frac{S}{P / T}+\varepsilon_{0}
$$

where:

$\varepsilon$ is the true full-energy peak efficiency

$S$ is the probability of producing a count in the sum peak (observed counts in sum peak divided

by photon-pairs emitted)

$P / T$ is the peak-to-total ratio of the coincident photon

$\varepsilon_{0}$ is the uncorrected full-energy peak efficiency

The final result can be interpreted as the final (true) efficiency being the sum of the uncorrected efficiency and a correction factor. This correction factor is the probability of a fullenergy peak deposition summing with the coincident photon. The term $S$ corresponds to the probability that the coincident photons were both emitted and that they both produced fullenergy pulses within the detector system. The $P / T$ in the denominator changes this factor in such a way that instead of finding the probability that both photons produced full-energy pulses, it finds the probability that one photon produced a full-energy pulse and the other produced 
any pulse at all that sums with the original photon's full-energy pulse (and that both photons were emitted coincidentally). It should be noted that the above technique is only valid for nuclides with a two-photon cascading emission. For more than one cascade, readers are directed elsewhere [30].

The variance in $S$ can be expressed as the variance of a quotient [4]:

$$
\sigma_{S}^{2}=\left(\frac{C_{S}}{t * A_{S}}\right)^{2}\left[\left(\frac{\sigma_{C_{S}}}{C_{S}}\right)^{2}+\left(\frac{\sigma_{A_{S}}}{A_{S}}\right)^{2}\right]
$$

where the uncertainty in the live time $t$ is assumed to be negligible compared to the uncertainty of the other terms.

The variance in the efficiency is given by a combination of the variance of a sum and the variance of a quotient [4]:

$$
{\sigma_{\varepsilon}}^{2}=\left(\frac{S}{P / T}\right)^{2}\left[\left(\frac{\sigma_{S}}{S}\right)^{2}+\left(\frac{\sigma_{P / T}}{P / T}\right)^{2}\right]+{\sigma_{\varepsilon_{0}}}^{2}
$$

where $\sigma_{i}^{2}$ is the variance in $i$.

\subsection{Example Using ${ }^{88} \mathrm{Y}$}

${ }^{88} \mathrm{Y}$ has two main gamma-ray emissions with energies of $898 \mathrm{keV}$ and $1836 \mathrm{keV}$. The decay scheme for this nuclide is shown in Figure 26 [46]. As can be seen, the nuclide decays to the second excited energy level $94.5 \%$ of the time, and the emission rate of the $898 \mathrm{keV}$ photon is $93.9 \%$. $5.23 \%$ of the time, the nuclide decays to the first level and only the $1836 \mathrm{keV}$ photon is emitted. It can be shown that the emission rate of the $898-1836 \mathrm{keV}$ pair is:

$$
\Gamma_{898,1836}=\frac{A I_{898} I_{1836}}{T_{1}}
$$

where:

$\Gamma_{898,1836}$ is the gamma-emission rate of the pair

$A$ is the activity of the sample

$I_{E}$ is the intensity of the gamma-emission of the photon with energy $E$

$T_{i}$ is the sum of intensities of all transitions to level $i$ 
Emission probabilities

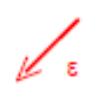

per 100 disintegrations
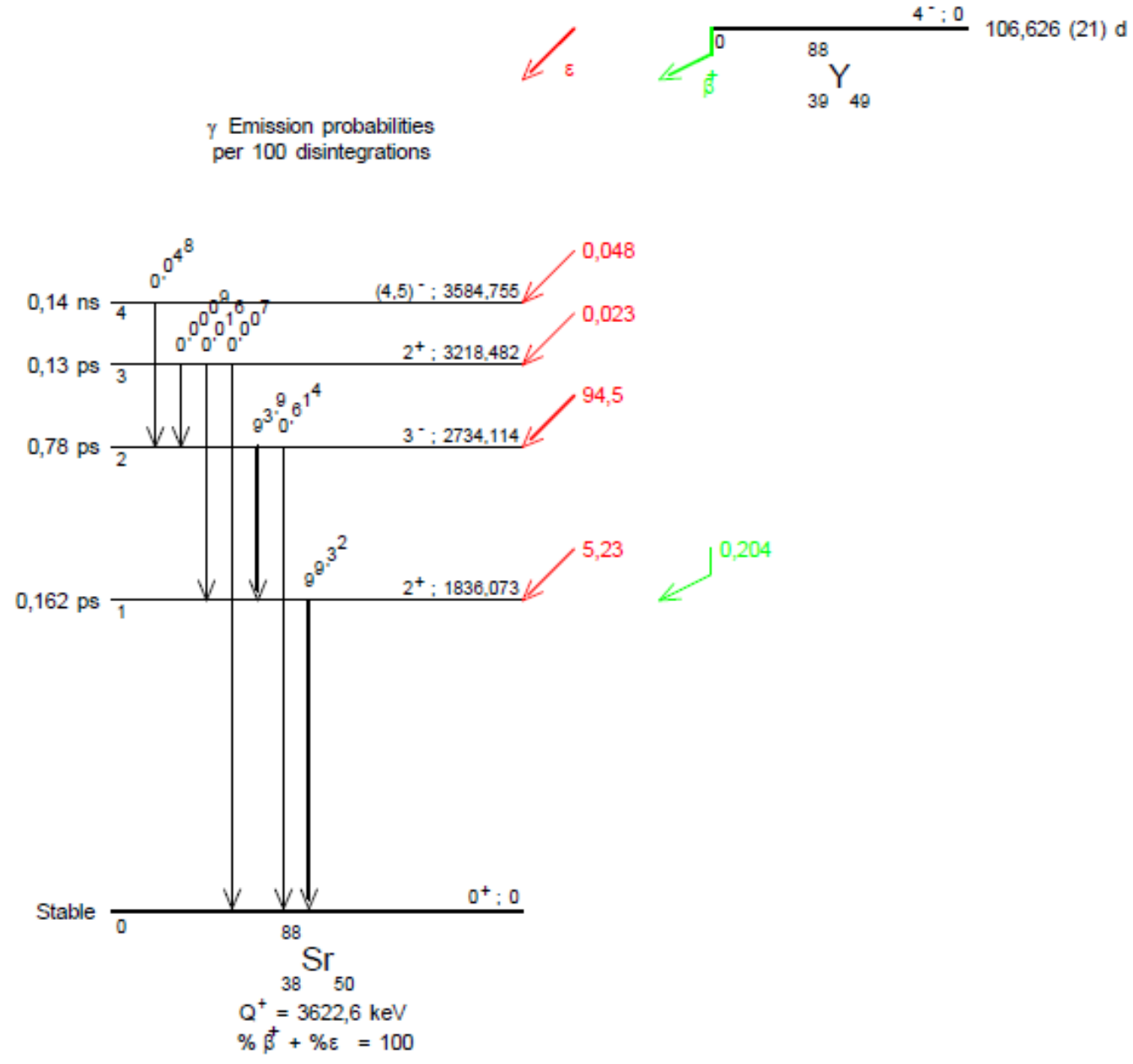

Figure 26: Decay scheme for Y-88 [46]

The term $A l_{898}$ also corresponds to $\Gamma_{898}$, the gamma-emission rate of the $898 \mathrm{keV}$ gamma-ray. Plugging in values for $I_{1836}$ and $T_{1}$, we have

$$
\Gamma_{898,1836}=\Gamma_{898} * .9994(4)
$$

For the Type 1 sample, the emission rate of the $898 \mathrm{keV}$ gamma-ray at the time of counting was 190.1(74) gammas/s. So the emission rate of the $898-1836 \mathrm{keV}$ pair at the time of counting was 190.0(74) gammas/s. The count rate in the sum peak was 2.66(1) counts/s in Add Back mode. Therefore, $S$ - the sum peak "efficiency" from the equation above - becomes $2.66(1) / 190.0(74)$ $=0.0140(5)$. The uncorrected efficiency for the $898 \mathrm{keV}$ gamma in Add Back mode is 0.088(3) and the $\mathrm{P} / \mathrm{T}$ ratio of the $1836 \mathrm{keV}$ gamma is $0.21925(20)$ in Add Back mode. Using the equation 
for efficiency involving cascading gamma-ray emissions, the corrected efficiency for the $898 \mathrm{keV}$ gamma becomes $0.152(6)$. Similarly, the efficiency for the $1836 \mathrm{keV}$ gamma rises from the uncorrected value of $0.048(2)$ to the corrected value of $0.093(4)$.

\subsection{Efficiency Curve Equation}

The efficiency curve has the following general form [47]:

$$
\varepsilon^{P}(E)=a_{0} E^{-a_{1}}+a_{2}-a_{3} e^{-a_{4} E}
$$

where:

$\varepsilon^{P}$ is the full-energy peak efficiency

$E$ is the gamma energy being analyzed in $\mathrm{MeV}$

$a_{0}-a_{4}$ are empirical constants to be found for each counting mode

Table 5 shows the corrected efficiencies for each observed gamma energy in Add Back mode using a Type 3 source.

Table 5: Peak Efficiency for Type 3 Source in Add Back mode

\begin{tabular}{|c|c|}
\hline Energy (keV) & Corrected Efficiency \\
\hline 59.54 & $0.477 \pm 0.009$ \\
\hline 88.0336 & $0.513 \pm 0.018$ \\
\hline 122.06 & $0.497 \pm 0.014$ \\
\hline 165.864 & $0.470 \pm 0.012$ \\
\hline 279.1967 & $0.321 \pm 0.025$ \\
\hline 391.69 & $0.281 \pm 0.007$ \\
\hline 661.66 & $0.200 \pm 0.006$ \\
\hline 898.042 & $0.168 \pm 0.007$ \\
\hline 1173.237 & $0.143 \pm 0.005$ \\
\hline 1332.501 & $0.131 \pm 0.005$ \\
\hline 1836.063 & $0.104 \pm 0.004$ \\
\hline
\end{tabular}

Using these points, the curve of best fit using the efficiency equation for Add Back mode becomes:

$$
\varepsilon^{P}(E)=0.157 E^{-0.634}-0.00150-2.203 e^{-26.4 E}
$$

where $E$ is the energy of the photon in MeV. The method for obtaining the error on predicted efficiency values fit is similar to that described in the peak fitting and $P / T$ ratio curve fits. Once 
again, the Fisher matrix is used to estimate the covariance matrix as described in Appendix 1. The Fisher matrix is therefore [45]:

$$
C_{j k}=\sum_{i=1}^{n} w_{i} \frac{\partial f}{\partial a_{j}} \frac{\partial f}{\partial a_{k}}
$$

The partial derivatives for the general equation of the peak efficiency are:

$$
\begin{aligned}
\frac{\partial f}{\partial a_{0}} & =E^{-a_{1}} \\
\frac{\partial f}{\partial a_{1}} & =-a_{0} \ln (E) E^{-a_{1}} \\
\frac{\partial f}{\partial a_{2}} & =1 \\
\frac{\partial f}{\partial a_{3}} & =e^{-a_{4} E} \\
\frac{\partial f}{\partial a_{4}} & =a_{3} E e^{-a_{4} E}
\end{aligned}
$$

The equation for the variance-covariance matrix is:

$$
V=C^{-1}
$$

Going through the algebra, the variance-covariance matrix for Add Back mode with a Type 3 source becomes:

$$
V=\left[\begin{array}{ccccc}
0.00108 & -0.00343 & -0.00102 & -0.00502 & 0.0948 \\
-0.00343 & 0.0112 & 0.00325 & 0.0142 & -0.345 \\
-0.00102 & 0.00325 & 0.00097 & 0.00471 & -0.0900 \\
-0.00502 & 0.0142 & 0.00471 & 0.0973 & 0.265 \\
0.0948 & -0.345 & -0.0900 & 0.265 & 17.8
\end{array}\right]
$$

Table 6 shows the data points for Singles mode. Fitting these points to the general equation in Singles mode gives:

$$
\varepsilon^{P}(E)=0.0814 E^{-0.949}+0.0149-3.91 e^{-28.3 E}
$$

and the variance-covariance matrix is:

$$
V=\left[\begin{array}{ccccc}
0.0000771 & -0.000609 & -0.0000685 & -0.00232 & 0.0126 \\
-0.000609 & 0.00511 & 0.000541 & 0.0173 & -0.127 \\
-0.0000685 & 0.000541 & 0.0000619 & 0.00204 & -0.0114 \\
-0.00232 & 0.0173 & 0.00204 & 0.165 & 0.0892 \\
0.0126 & -0.127 & -0.0114 & 0.0892 & 6.14
\end{array}\right]
$$


Figure 27 and Figure 28 show the observed data points and fitted curves for the Add Back mode and Singles mode efficiency calculations.

Table 6: Peak efficiency for Type 3 source in Singles mode

\begin{tabular}{|c|c|}
\hline Energy (keV) & Corrected Efficiency \\
\hline 59.54 & $0.473 \pm 0.009$ \\
\hline 88.0336 & $0.510 \pm 0.018$ \\
\hline 122.06 & $0.486 \pm 0.014$ \\
\hline 165.864 & $0.430 \pm 0.011$ \\
\hline 279.1967 & $0.292 \pm 0.013$ \\
\hline 391.69 & $0.211 \pm 0.005$ \\
\hline 661.66 & $0.133 \pm 0.004$ \\
\hline 898.042 & $0.109 \pm 0.003$ \\
\hline 1173.237 & $0.086 \pm 0.002$ \\
\hline 1332.501 & $0.077 \pm 0.002$ \\
\hline 1836.063 & $0.060 \pm 0.002$ \\
\hline
\end{tabular}

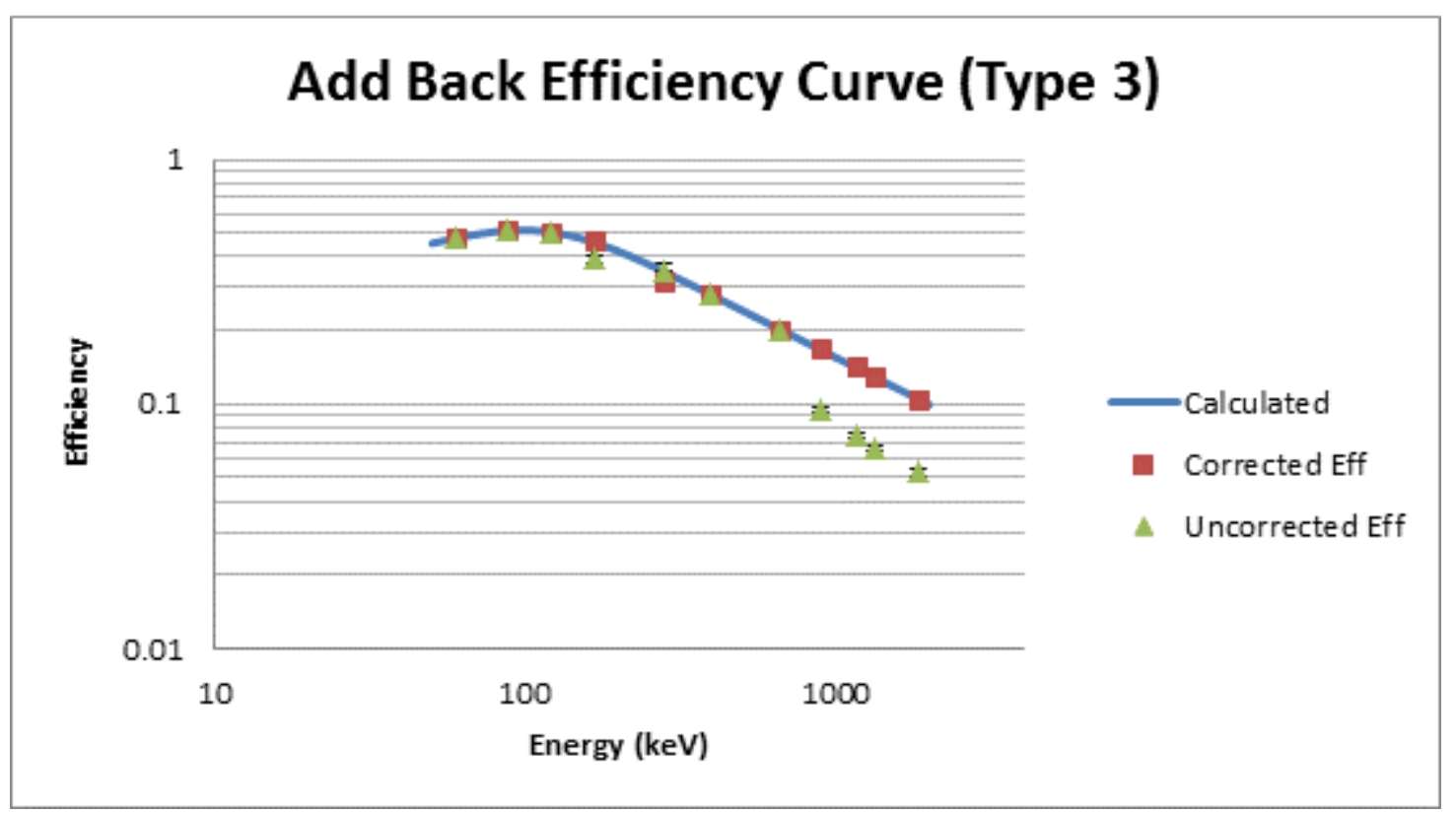

Figure 27: Efficiency curve for Type 3 source in Add Back mode 


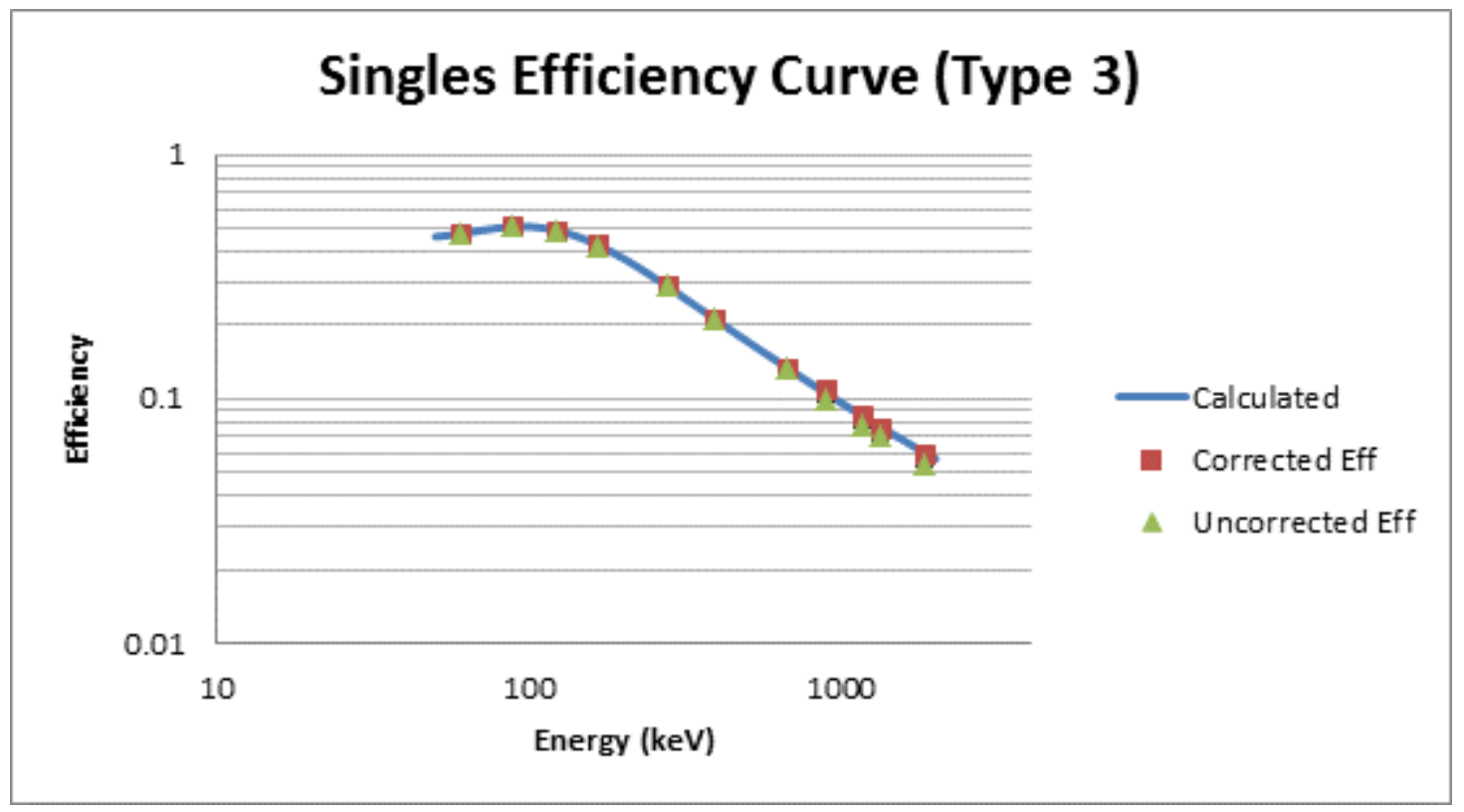

Figure 28: Efficiency curve for Type 3 source in Singles mode

Comparing the efficiency curves between Add Back mode and Singles mode, it should be noted that the curves are very similar up to about $300 \mathrm{keV}$, beyond which the Singles mode efficiency drops off more dramatically than Add Back mode. This suggests that the advantages of Add Back mode are more significant at higher energies (above $300 \mathrm{keV}$ ). Also, the difference between corrected and uncorrected efficiency data points are more pronounced in Add Back mode than in Singles mode. This is to be expected due to the summing of coincident photons between crystals in Add Back mode, as opposed to Singles mode which only sums coincident photons within a single crystal.

Taking these facts into consideration, if nuclides emitting cascading gammas are to be analyzed using this system, then Singles mode may be more useful than Add Back mode in observing photons below $300 \mathrm{keV}$, especially if cascading gamma-ray emissions are involved. This is because the efficiency of the system is no different in Singles mode than Add Back mode in this energy range. If energies above $300 \mathrm{keV}$ are to be observed, researchers using this system should be aware of the advantages and disadvantages of using Singles and Add Back modes. 


\subsection{Efficiency of gamma-gamma coincidence mode}

The efficiency of gamma-gamma coincidence mode is a bit more complicated due to the fact that the efficiency is dependent on the energies of the two photons being emitted coincidentally. Gamma-gamma coincidence mode is operated such that an event is recorded when an interaction detected in four-crystal Add Back mode in one clover detector is found to be in coincidence with an interaction detected in four-crystal Add Back mode in the other clover detector. Therefore, the efficiency of gamma-gamma coincidence mode is the product of the efficiency of the first gamma-ray energy in one clover detector and the efficiency of the second gamma-ray energy in the other clover detector:

$$
\varepsilon_{C}=\varepsilon_{1}\left(E_{1}, E_{2}\right)=\varepsilon_{1}\left(E_{1}\right) \varepsilon_{2}\left(E_{2}\right)
$$

where:

$\varepsilon_{C}$ is the efficiency of the gamma-gamma coincidence event

$\varepsilon_{i}\left(E_{i}\right)$ is the efficiency of the $i^{\text {th }}$ clover detector operated in four-crystal Add Back mode at energy $E_{i}$

Therefore, it is necessary to also calculate the efficiency curves for each clover detector when operated in four-crystal Add Back mode. The two clover detectors are named Misty and Kerri in order to keep them distinguishable. Table 7 shows the data points for the corrected efficiencies when using the system in these two modes of operation. Figure 29 and Figure 30 show the efficiency curves for these two modes as well. 
Table 7: Efficiency data points for Misty and Kerri Four-Crystal Add Back mode

\begin{tabular}{|c|c|c|}
\hline Energy (keV) & Misty Efficiency & Kerri Efficiency \\
\hline 59.54 & $0.263 \pm 0.005$ & $0.210 \pm 0.004$ \\
\hline 88.0336 & $0.276 \pm 0.010$ & $0.234 \pm 0.008$ \\
\hline 122.06 & $0.263 \pm 0.008$ & $0.227 \pm 0.007$ \\
\hline 165.864 & $0.239 \pm 0.006$ & $0.206 \pm 0.005$ \\
\hline 279.1967 & $0.176 \pm 0.008$ & $0.164 \pm 0.016$ \\
\hline 391.69 & $0.137 \pm 0.003$ & $0.122 \pm 0.003$ \\
\hline 661.66 & $0.0956 \pm 0.003$ & $0.0855 \pm 0.002$ \\
\hline 898.042 & $0.0835 \pm 0.003$ & $0.0720 \pm 0.002$ \\
\hline 1173.237 & $0.0678 \pm 0.002$ & $0.0594 \pm 0.002$ \\
\hline 1332.501 & $0.0617 \pm 0.002$ & $0.0542 \pm 0.002$ \\
\hline 1836.063 & $0.0496 \pm 0.002$ & $0.0427 \pm 0.001$ \\
\hline
\end{tabular}

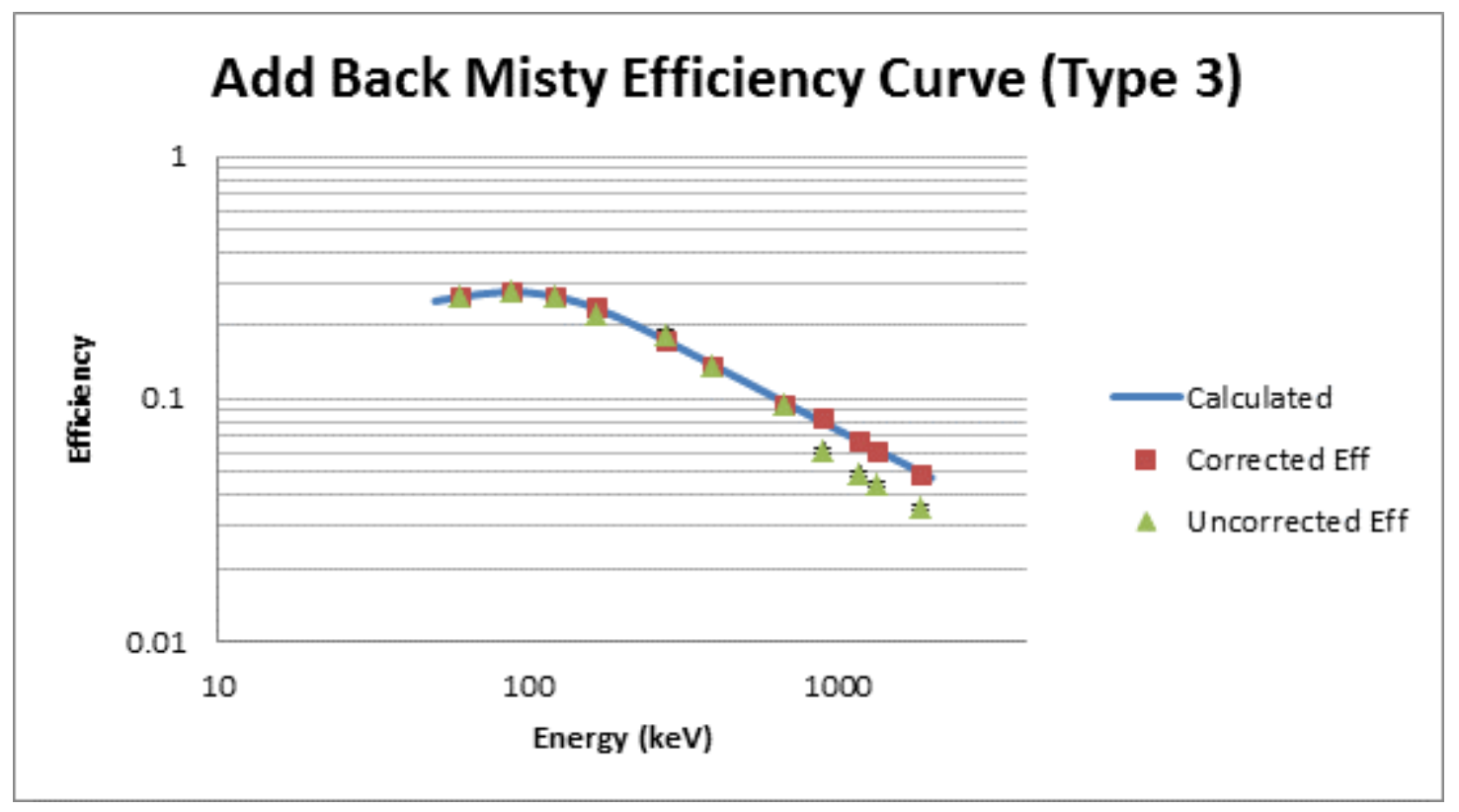

Figure 29: Efficiency curve for Type 3 source in Misty Add Back mode 


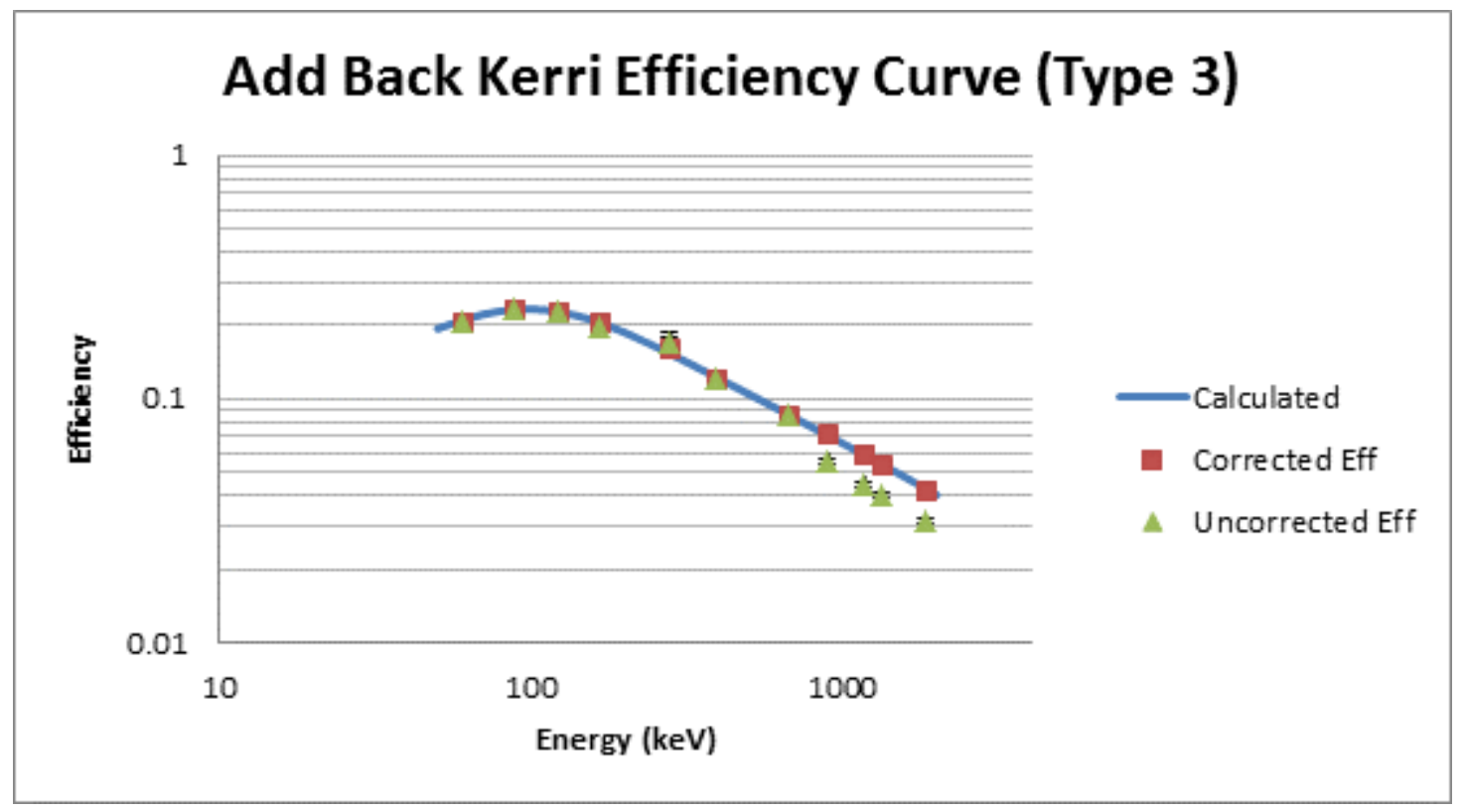

Figure 30: Efficiency curve for Type 3 source in Kerri Add Back mode

Figure 31 shows a zoomed-in display of the 2-dimensional gamma-gamma coincidence graph of the Type 3 source to show the coincidences between the photons of ${ }^{60} \mathrm{Co}$. A projection can be produced from this graph so that the events from Misty are plotted with the condition that they are in coincidence with an event from Kerri that had an energy between 1171 and $1175 \mathrm{keV}$. This will produce a one-dimensional spectrum that can be analyzed for the peak area of the gamma-gamma coincidence events between Misty and Kerri with ${ }^{60} \mathrm{Co}$.

Misty-Kerri Coincidences

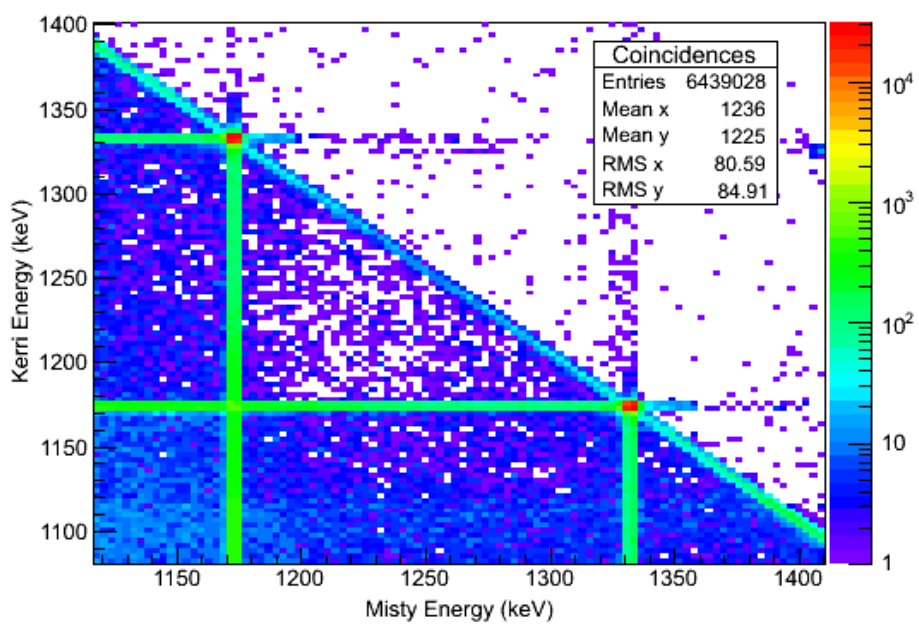

Figure 31: Co-60 in gamma-gamma coincidence mode 


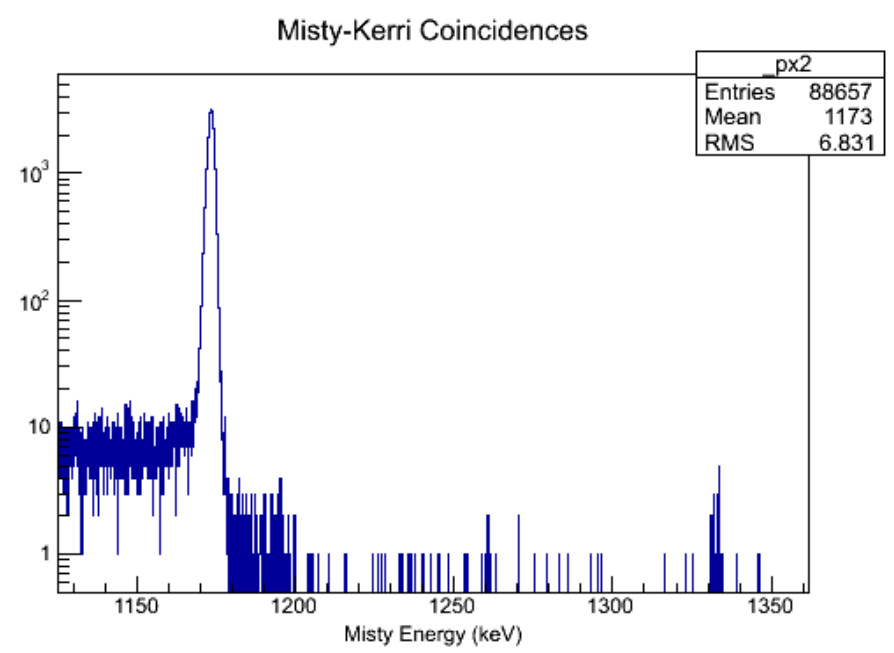

Figure 32: Spectrum obtained by gating Kerri on $1333 \mathrm{keV}$

Figure 32 shows the one-dimensional projection of the events detected by Misty when an event around $1333 \mathrm{keV}$ was detected in Kerri. The dead-time corrected area of the peak is $110930 \pm 361$ counts. The spectrum was taken for 60000 seconds, making the detected count rate of gamma-gamma coincidence events to be $1.85 \pm 0.01 \mathrm{cps}$. The emission rate of 1173 $1333 \mathrm{keV}$ gamma-ray pairs for the sample is $517 \pm 13$ pairs per second. The efficiency of the system for this pair in gamma-gamma coincidence mode is therefore $0.00358 \pm 0.00009$ or $0.358(9) \%$. The efficiency of Misty four-crystal Add Back mode at $1173 \mathrm{keV}$ is $0.0678 \pm 0.0023$ and the efficiency of Kerri four-crystal Add Back mode at $1333 \mathrm{keV}$ is $0.0542 \pm 0.0017$. The product of these two efficiencies is $0.00368 \pm 0.00017$ or $0.368(17) \%$. The theoretical and observed efficiencies agree to within $1 \sigma$.

The same type of analysis can be performed with ${ }^{88} \mathrm{Y}$. By gating around $1836 \mathrm{keV}$ on Kerri, the $898 \mathrm{keV}$ peak is clearly visible in Misty's spectrum. The dead-time corrected area of the 898 peak in this projected spectrum is $10630 \pm 141$ counts. This was taken in a 60000 second spectrum, so the rate of $898-1836 \mathrm{keV}$ gamma-ray pairs detected by the system is 0.177 $\pm 0.002 \mathrm{cps}$. The emission rate of $898-1836$ pairs from the sample is $53 \pm 1$ pairs per second, so the efficiency of the system in detecting this photon pair is $0.00334 \pm 0.00007$ or $0.334(7) \%$. The efficiency of Kerri detecting the $1836 \mathrm{keV}$ gamma-ray is $0.0427 \pm 0.0013$ and the efficiency of Misty detecting the $898 \mathrm{keV}$ gamma-ray is $0.0835 \pm 0.0029$. The product of these two efficiencies should equal the efficiency of detecting the pair in gamma-gamma coincidence mode. Indeed, the product of these efficiencies is $0.00357 \pm 0.00012$ or $0.357(12) \%$, which 
agrees with the observed efficiency of $0.334(10) \%$ to within $2 \sigma$. These results may be improved if angular correlation effects were taken into account. 


\section{Chapter 8: Dead-Time}

Dead-time is inherent in all detector systems due to the inability of the electronics to properly process events while processing previous pulses [4]. In all systems, there is a minimum amount of time necessary to process an event before being able to accept another event. In digitizer systems, there may be considerable additional dead-time due to the transfer of data from the buffer to the external file. Both of these types of "dead-time" must be accounted for separately. To initiate a count using this particular system, users specify the "Live Time" for which they want the system to take data. The Live Time variable in the Pixie-4 system is defined as the length of time the system is ready to accept events, whether or not an event is already being processed [15]. The Live Time variable only omits times that the ADC is out of range, overloaded with processing, or transferring memory. It is the amount of time that channels can accept triggers for recording or pileup of data. The time the digitizers spend shaping pulses and recording data is not saved in the current method of the data collection, and thus must be calculated in other ways.

\subsection{Dead-Time Due to Data Transfer}

The dead-time incorporated in the system due to data transfer can be calculated from the difference in elapsed time (time difference from the start of the count to the end of the count) and the Live Time variable output. The elapsed time is important for calculating the decay of nuclides during counting. If the nuclides of interest have half-lives that are much longer than the counting time, then the elapsed time can be ignored and only the live time should be used. The Pixie-4 digitizers have three ways of storing and transferring data. They are Single Buffer mode, 32 Buffer mode, and 16/16 Buffer mode [15]. Single Buffer mode collects data and stores it in an $8 \mathrm{~KB}$ buffer until the buffer becomes full. It then stops recording data while it outputs the data to the external file, which takes about $30 \mathrm{~ms}$. During this dumping of the data, the system is dead to any incoming pulses and the onboard Live Time variable is paused [15]. 32 Buffer mode uses 32 buffers that are each $8 \mathrm{~KB}$. When one buffer is full, it transfers its data to a segment of external memory, which takes about $300 \mu \mathrm{s}$. When 32 buffers have been transferred to external memory, the contents of the external memory are dumped to the external data file, which takes about 30 ms [15]. 16/16 Buffer mode acts like 32 Buffer mode, except when 16 buffers are full, the system transfers those 16 buffers to the external 
data file while the system continues filling the remaining 16 buffers [15]. Ideally then, the only dead-time incurred will be the transfer of each buffer to the external memory (300 $\mu$ s per buffer) and the $30 \mathrm{~ms}$ dead-time will be skipped. However, if the system incurs a high count rate, the 16 buffers may fill up before the other 16 buffers have finished dumping their data. In this case, the system must wait until the first 16 buffers have finished dumping their data before dumping the next 16 buffers [15]. Using Single Buffer mode, the system will incur about $32 \times 30$ = $960 \mathrm{~ms}$ of dead-time in transferring 32 buffers of data. In 32 Buffer mode, the system will incur $32 \times 0.3+30=39.6$ ms of dead-time in transferring 32 buffers of data. In 16/16 Buffer mode, the system will incur $32 \times 0.3=9.6 \mathrm{~ms}$ of dead-time in transferring 32 buffers of data (assuming there is no interruption due to high data collection rates). The dead-time incurred by using Single Buffer mode is therefore about 100 times higher than by using 16/16 Buffer mode [15].

In almost all scenarios, the 32 Buffer mode or 16/16 Buffer mode should be used for data collection; however, the interaction between the host PC and the Pixie-4 digitizers in this system created an unknown error when trying to use 32 Buffer mode or 16/16 Buffer mode. This may have to do with the interaction between the detector system and the Linux operating system, but no fixes have yet been found for this error. Because of this, Single Buffer mode was used for the characterization of the system and analysis of the irradiated uranium samples. This should not affect the system in a significant way, but it will cause more dead-time in the system due to data transfer.

\subsection{Dead-Time Due to Pulse Shaping and Trace Collection}

The dead-time due to pileup occurrences is described in Section 4.1. Because radioactive decay is a random process, pileup events will occur according to Poisson statistics [4]. If two triggers are issued within the shaping time in the same crystal, then the events will be rejected and the system will be considered dead for the entire time. Interactions that are rejected in this way will result in the omission of two (or more) events. For this work, the shaping time and thus the minimum peak separation time is $13.2 \mu \mathrm{s}$.

In Add Back mode, if more than one interaction occurs within the resolution time of the system, the energies attributed to the interactions will be summed and plotted in the spectrum. Two events can "pileup" in Add Back mode and not be rejected by the pileup inspector because 
they happen in two different crystals. Currently, there is no post-processing pileup rejection in the software. At high count rates, this could have significant impacts on the Add Back spectrum. Another scenario that should be addressed is if a multi-crystal interaction from one photon occurs, and one of those crystals has a pileup interaction, then events only in that crystal will be rejected and the remaining crystals will report the energy deposited in them. This could lead to partial energy recordings being plotted in Add Back mode even though the full energy of the photon was deposited in the system. This would affect the efficiency of the system if this type of interaction occurred often enough. Photons of higher energies are more susceptible to these types of pileup interactions, because they have a higher likelihood of interacting in more than one crystal. In the most recent release of the Pixie-4 software (version 2.51), a flag can be recorded in an event indicating that a pileup occurred in one of the channels. This could be useful in future updates to the post-processing program for the clover system in determining whether events should be rejected due to pileup in one of the channels. This type of interaction can also interfere with gamma-gamma coincidence mode, because a pileup can occur in one detector, but not the other.

Another factor in the dead-time is the time it takes for the system to transfer data about the event to the buffer. If the data being transferred only involves information about the event, and does not include waveforms, then the system can continue analyzing pulses while the data about the event is being transferred. This is because the information about the event (e.g. hit patterns and timestamps) is stored separately from the waveform data, which is used to shape incoming pulses. If waveforms are to be recorded, then the system is unable to overwrite the waveform data before it is transferred. In this case, additional dead-time will be incurred. In general, the data about the event takes about 850 ns of overhead, plus about 600 ns per channel in the hit pattern to transfer [15]. For waveform data, it generally takes about three times as long to write the waveform data as it does to read it [15]. For example, a trace length of $3 \mu$ s will take about $9 \mu$ s to write to the buffer. In this scenario, the system must stop collecting waveform data while it is waiting for the data to be written to the buffer. While the system is waiting, additional events will not be recorded. Therefore, additional dead-time due to data transfer and pulse trace collection is on the order of $1.5 \mu$ s to record the hit pattern data for the event plus 3 times the trace length. When data has been transferred to the buffer, 
information from all channels are cleared in the module, which causes dead-time in all channels after a hit is recorded in the module, whether or not they contributed to the hit pattern. If a hit occurs in any channel after the Coincidence Window and before the waveform data has been completely transferred, the hit will not be registered in the system.

For this work, waveforms were not used; however, a default setting was not changed, causing trace lengths of $3.5 \mu$ s to be recorded for each pulse. This added about $10.5 \mu$ s of deadtime in each channel per module event (a module event can consist of multiple channel hits). An interaction that is not recorded because of dead-time due to data transfer only causes the omission of one event, as opposed to pulse pileup rejection which causes the omission of at least two events.

\subsection{Calculating Dead-Time}

Taking all of this into consideration, the Live Time output of the system must be corrected for the dead-time due to the above factors. Calculating the dead-time for multiple detector systems is more complex than calculating the dead-time for single detector systems because one part of the system can be available to take data while another part of the system is dead. To take this into account, the dead-time must be calculated for each individual crystal. From this, the probability that a photon will interact with a dead crystal can be calculated.

In a traditional analog system with pileup rejection, it has been shown that the fraction of true events that escape pileup in a single crystal can be written as [4]:

$$
f_{1}=e^{-2 n_{1} \tau_{1}}
$$

where:

$n_{1}$ is the true count rate per crystal

$\tau_{1}$ is the minimum time interval needed between hits for the event to pass the pileup inspector.

In this case, $\tau_{1}$ is $13.2 \mu \mathrm{s}$. In the case of counting modes that utilize multiple detectors for binning (e.g. Add Back and gamma-gamma coincidence modes), Equation (8.1) should be modified to:

$$
F_{1}=e^{-\left(2 n_{1} \tau_{1}\right) i}
$$

where $i$ is the average number of crystals that the photon(s) will interact with. In Add Back mode, $i$ will be energy dependent and can range from one to eight; in gamma-gamma 
coincidence mode, $i$ can range from two to eight and will depend on both energies involved in the coincidence; in Singles mode, $i$ will always equal 1.

For dead-time incurred by a detector crystal due to data transfer to the buffer (including trace collection), the detector can be assumed to be "off" at these points. The fraction of true events that will not interact with a detector crystal while data is being transferred can therefore be written as:

$$
f_{2}=e^{-4 n_{2} \tau_{2}}
$$

where:

$n_{2}$ is the event rate per module

$\tau_{2}$ is the dead-time incurred by each channel in a module per event

There is a factor of four at the beginning because each module event causes dead-time in four crystals. For counting modes that utilize multiple modules (e.g. Add Back or gamma-gamma coincidence mode), the fraction of true events that will not interact with the system while data is being transferred will be:

$$
F_{2}=e^{-\left(4 n_{2} \tau_{2}\right) j}
$$

where $j$ is the average number of modules that the photon(s) will interact with. In Add Back mode $j$ can range from one to two. In gamma-gamma coincidence mode, $j$ will always be two. In Singles mode, $j$ will always be one. Notice that $n_{1}$ and $n_{2}$ differ significantly. The $n_{1}$ term describes the true count rate per channel while $n_{2}$ describes the event rate per module. An event can have up to four channel counts - one from each channel. The reason for specifying between $n_{1}$ and $n_{2}$ is because pileup rejection only affects the crystal that the pileup occurs in, but the event data transfer dead-time affects the entire module. Any events that occur after the coincidence window and before the end of the data transfer are lost because all channels are cleared in a module after the data has been transferred to the buffer. The final equation for the fraction of events lost to pileup or interaction with a dead crystal is:

$$
F=F_{1} F_{2}=e^{-\left(2 n_{1} \tau_{1} i+4 n_{2} \tau_{2} j\right)}
$$

If the fraction of lost events is close to zero $(<10 \%)$, then this equation can be approximated by:

$$
F \cong 1-\left(2 n_{1} \tau_{1} i+4 n_{2} \tau_{2} j\right)
$$




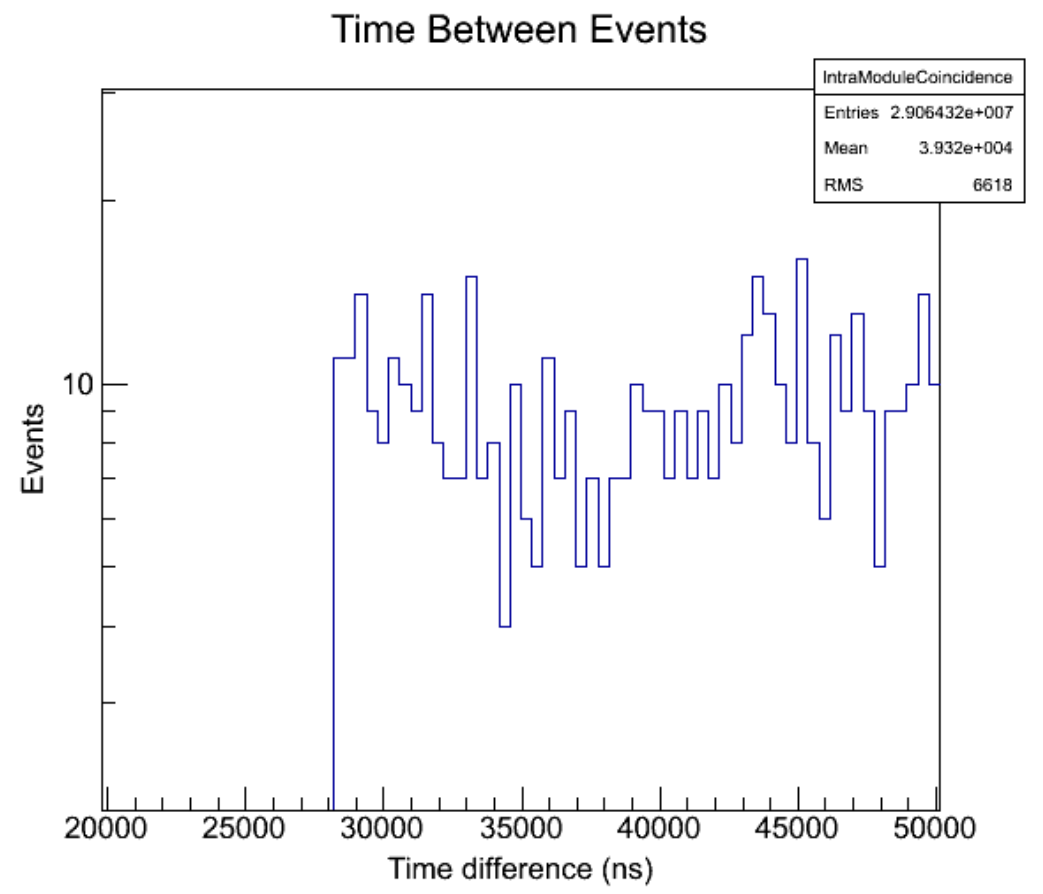

Figure 33: Amount of time between events

and $n_{1}$ and $n_{2}$ can be approximated by the observed count rates, as opposed to the input count rates. The actual value of the trace length being recorded in each spectrum is unknown, but a diagnostic plot can be created in which the time between events within the same module can be displayed. A zoomed-in display of this plot is shown in Figure 33. As displayed, the minimum time between events is observed to be about $28.2 \mu \mathrm{s}$. The shaping time for each event is 13.2 $\mu \mathrm{s}$, so the time it takes to transfer the event data and the waveform data is about $15.0 \mu \mathrm{s}$. If the event data takes $1.5 \mu \mathrm{s}$ to transfer, then the waveform data takes $13.5 \mu \mathrm{s}$, indicating that the trace length is about $4.5 \mu$ s per event (because the dead-time incurred from trace capture is about three times as long as the length of the trace).

Ideally, no waveforms would be collected if pulse-shape analysis is not being performed. The dead-time in these circumstances is usually dominated by the filter dead-time. For data collection in which no waveforms are being collected and the system is experiencing a relatively small count rate, the fraction of events lost in each detector crystal is approximated as [4]:

$$
f \cong 1-2 n_{1} \tau_{1} i
$$

whereas if waveforms are being collected, the fraction of events lost in each detector crystal is approximated as: 


$$
f \cong 1-\left(2 n_{1} \tau_{1} i+4 n_{2} \tau_{2} j\right)
$$

The values for $\tau_{1}$ and $\tau_{2}$ are easily calculated by the settings in the system; however, the values for $n_{1}$ and $n_{2}$ are dependent on the sample being counted. In this work, every effort was taken to ensure that the sample was as close to the center of the system as possible. In addition, all the crystals have very similar specifications in terms of efficiency and shape. Combining these reasons with the fact that the count rates in the crystals are relatively low, it is reasonable to assume that $n_{1}$ can be approximated by the average count rate observed in each crystal. This can be determined by taking the total count rate observed in Singles mode and dividing by 8 . Similarly, $n_{2}$ can be approximated by the average event rate observed in the two modules. This is determined by taking the average of the count rates in the Add Back Misty spectrum and the Add Back Kerri spectrum. A count in one of these spectra represents the sum of the overall energy recorded in each event, and so the total number of events observed in these spectra represents the number of events recorded by the modules.

Here, $i$ represents the average number of crystals that a photon will cause triggers in when it deposits its full energy in the system (i.e. causes a full-energy peak count in Add Back mode). This will be energy dependent and can be calculated for each photon of interest by post-processing the List Mode file. By gating on a certain energy and counting the number of total events and the total number of detectors that were hit during those events, the average number of detectors per interaction can be calculated. This will not be applicable if there is significant summing-in because this type of interaction involves two photons and will significantly increase the likelihood that more than one crystal will be involved in the interaction. For peaks with significant summing-in, either a nearby peak in the same spectrum that is not subject to summing-in may be used or interpolation may be used to estimate $i(E)$. The function $i(E)$ is a property of the detector system and the sample geometry, and thus should be the same between samples of similar geometry. Figure 34 shows $i(E)$ in Add Back mode for the peaks used in the efficiency calibration spectrum. Note, if calculating $i(E)$ for Add Back mode, all events that sum to $E$ should be considered; however, if calculating for gamma-gamma coincidence mode, then the calculation of $i(E)$ should only consider events that occur completely in one clover detector. For example, if calculating $i(E)$ for the coincidence between the $1173 \mathrm{keV}$ photon and $1333 \mathrm{keV}$ photon in ${ }^{60} \mathrm{Co}$ with the $1173 \mathrm{keV}$ photon gated on Misty, then $i(E)$ should 


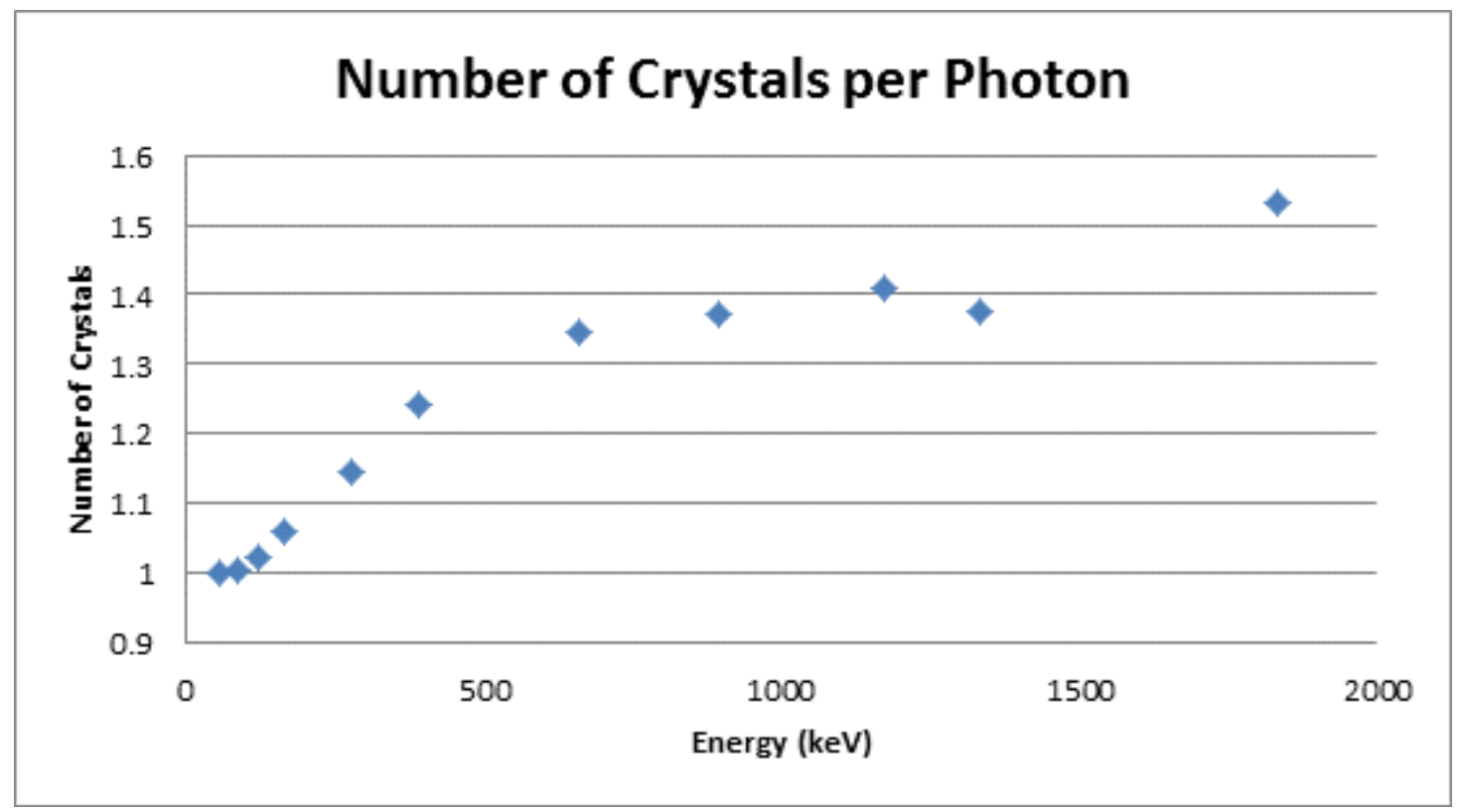

Figure 34: Average number of crystals photon deposits energy into

be calculated by looking at each photon separately. The term $i(E)$ will be the sum of the average number of detectors hit in $1173 \mathrm{keV}$ peak events in Add Back Misty and the average number of detectors hit in $1333 \mathrm{keV}$ peak events in Add Back Kerri. The most that $i(E)$ can be is 8, where all four crystals are hit from both photons in the coincidence.

The term $j$ represents the average number of modules that a photon interacts with. In Add Back mode, this will also be energy dependent and can be calculated from the efficiency curves for Add Back mode, Add Back Misty, and Add Back Kerri. By adding the efficiencies of Add Back Misty and Add Back Kerri together, the result is the probability that a photon will deposit its entire energy in one clover (i.e. one module). The efficiency for Add Back is the probability that a photon will deposit its full energy in the system as a whole (i.e. one or both modules). The average number of modules that a photon deposits its energy into can therefore be described by:

$$
j(E)=\frac{\varepsilon_{A B}(E)}{\varepsilon_{A B, M}(E)+\varepsilon_{A B, K}(E)}
$$

where:

$\varepsilon_{A B, M}(E)$ is the full-energy peak efficiency of Add Back Misty mode

$\varepsilon_{A B, K}(E)$ is the full-energy peak efficiency of Add Back Kerri mode

$\varepsilon_{A B}(E)$ is the full-energy peak efficiency of Add Back mode 
The efficiency curves for Add Back Misty and Add Back Kerri modes were calculated following the procedure outlined in Section 7.1. The coefficients are displayed in Table 8. A graph of $j(E)$ in Add Back mode is displayed in Figure 34.

Table 8: Efficiency coefficients for Add Back Misty and Add Back Kerri modes.

\begin{tabular}{|c|c|c|}
\hline Efficiency Coefficient & Add Back Misty & Add Back Kerri \\
\hline $\mathrm{a}_{0}$ & 0.07193 & 0.07087 \\
\hline $\mathrm{a}_{1}$ & 0.65529 & 0.60451 \\
\hline $\mathrm{a}_{2}$ & -0.00108 & -0.00848 \\
\hline $\mathrm{a}_{3}$ & 1.1787 & 1.0832 \\
\hline $\mathrm{a}_{4}$ & 29.251 & 29.893 \\
\hline
\end{tabular}

Because of the energy dependence of $i$ and $j$, the dead-time is also energy dependent (except Singles mode where both $i$ and $j$ are exactly 1.0). If the dead-time is already low, then $i$ and $j$ can be approximated as 1.0 without much uncertainty, but if the dead-time for the system is significant, then $i$ and $j$ should be calculated for each photon energy of interest.

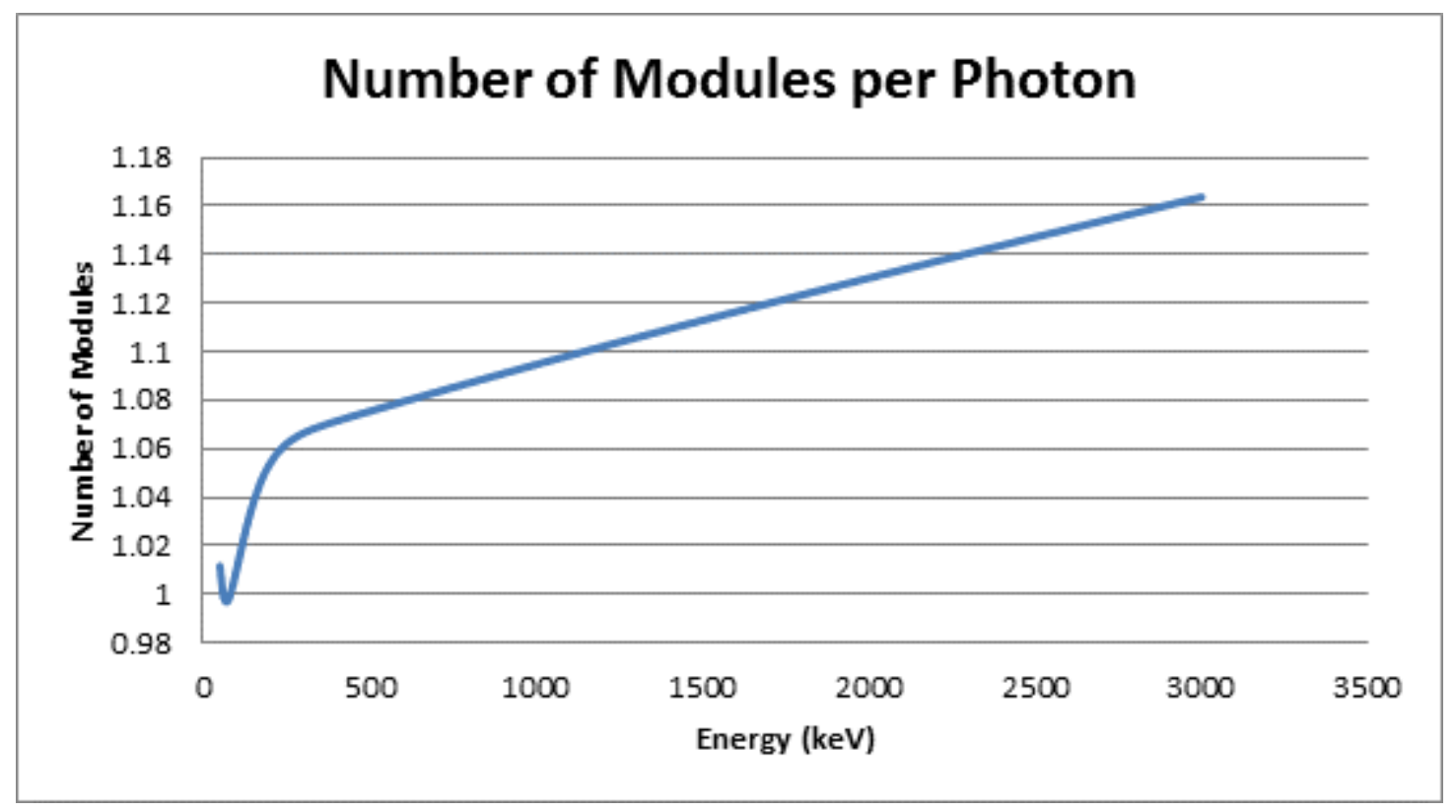

Figure 35: Average number of modules photon deposits energy into 


\section{Part 4: Analysis of Irradiated Uranium Samples}

\section{Chapter 9: Samples Used and Procedure}

In order to test the system's use for nuclear forensics purposes, two highly enriched ( 93\%) uranium foils were irradiated in the thermal research reactor located at the Massachusetts Institute of Technology for 12,000 seconds each. The samples were then dissolved in a $4 \mathrm{M}$ nitric acid solution. For each of the samples, $30 \mu \mathrm{L}$ of the solution were sandwiched between two thin pieces of Mylar and attached to a sample holder. The aliquot from the first sample was given ID number 4126-01-002 (or 4126 for short), and the aliquot from the second sample was given ID number 4130-01-002 (or 4130 for short). These two samples will simply be referred to as 4126 and 4130 .

Fission products decay through a fission product chain and eventually end at a stable nuclide. Commonly, near the end of the chain, there are two or more fission products with relatively long half-lives (longer than a few minutes). These are termed fission product parentdaughter pairs because each of these fission products can be measured for an appreciable length of time after irradiation. Depending on the half-lives of the fission products and the time since production, determining activities of these fission product pairs may give insight into useful properties of the sample. These properties include the age of the sample, characteristics of the sample before neutron bombardment occurred, and the nature of the neutron flux to which the sample was subjected. The fission product pairs chosen for analysis in this work are ${ }^{99} \mathrm{Mo} /{ }^{99 \mathrm{~m}} \mathrm{Tc},{ }^{140} \mathrm{Ba} /{ }^{140} \mathrm{La}$, and ${ }^{95} \mathrm{Zr} /{ }^{95} \mathrm{Nb}$.

\subsection{Counting Procedure and Spectrum Analysis}

The 4126 sample had a zero time (end of bombardment time) of 41.86632012 GMT.

These time units are given as the fractional Julian day of the year in 2012 GMT (e.g. January 1 at noon is 1.5000). The 4126 sample was counted according to the schedule shown in Table 9. The 4130 sample had a zero time of 119.1552012 GMT, and the counting schedule is shown in Table 10. 
Table 9: Counting Schedule of Sample 4126

\begin{tabular}{|c|c|c|}
\hline Time of Count (End) & Days After Zero Time & Count Live Time (s) \\
\hline 61.3625 & 19.4962 & 50400 \\
\hline 69.2125 & 27.3462 & 36000 \\
\hline 73.5751 & 31.7088 & 43200 \\
\hline 80.6659 & 38.7996 & 60000 \\
\hline 95.3634 & 53.4971 & 120000 \\
\hline 105.2535 & 63.3872 & 120000 \\
\hline 110.1099 & 68.2436 & 120000 \\
\hline 112.4124 & 70.5461 & 120000 \\
\hline 137.1426 & 95.2763 & 160619 \\
\hline 170.0509 & 128.1846 & 120000 \\
\hline 204.1254 & 162.2591 & 180000 \\
\hline 231.8514 & 189.9851 & 180000 \\
\hline
\end{tabular}


Table 10: Counting Schedule of Sample 4130

\begin{tabular}{|c|c|c|}
\hline Time of Count (End) & Days After Zero Time & Count Live Time (s) \\
\hline 123.754 & 4.599 & 6000 \\
\hline 124.078 & 4.923 & 12000 \\
\hline 125.146 & 5.991 & 16280 \\
\hline 126.235 & 7.080 & 19260 \\
\hline 128.143 & 8.988 & 24000 \\
\hline 128.982 & 9.827 & 26769 \\
\hline 131.087 & 11.932 & 30000 \\
\hline 132.303 & 13.148 & 30000 \\
\hline 133.418 & 14.263 & 30000 \\
\hline 134.350 & 15.195 & 30000 \\
\hline 138.257 & 19.102 & 45000 \\
\hline 139.556 & 20.401 & 45000 \\
\hline 140.285 & 21.130 & 45000 \\
\hline 143.230 & 24.075 & 45000 \\
\hline 144.563 & 25.408 & 45000 \\
\hline 145.476 & 26.321 & 45000 \\
\hline 146.324 & 27.169 & 45000 \\
\hline 151.504 & 32.349 & 60000 \\
\hline 158.726 & 39.571 & 60000 \\
\hline 161.696 & 42.541 & 60000 \\
\hline 166.673 & 47.518 & 60000 \\
\hline 171.692 & 52.537 & 60000 \\
\hline 175.355 & 56.200 & 60000 \\
\hline 180.375 & 61.220 & 120000 \\
\hline 183.388 & 64.233 & 120000 \\
\hline 189.380 & 70.225 & 120000 \\
\hline 197.408 & 78.253 & 120000 \\
\hline 211.306 & 92.151 & 120000 \\
\hline
\end{tabular}

It should be mentioned that some of the counts were stopped prematurely by the computer due to file size limitations. This resulted in the Live Time not being recorded correctly for these files. The only affected file from the 4126 sample was the count that ended at 137.1426. The affected files from the 4130 sample were the counts that ended at 125.146, 126.235, and 128.982. The Real Time was extracted from these files by comparing the timestamps between the first and last recorded events. The Live Time was estimated by interpolating the ratio of Live Time to Real Time for the files surrounding the accounts and calculating the Live Time based on the Real Time of the affected files. 
The samples were then run through the parsing procedure created by Bob Rundberg and edited by Steven Horne described in Chapter 5. This procedure creates a ROOT file that contains the spectra of the count for all modes of counting (Add Back, Singles, gamma-gamma coincidence, etc...). The Add Back and Singles mode spectra were then converted to a format compatible with Canberra's Genie 2000 software. Genie 2000 was used to locate the peaks in each spectrum and calculate the net peak areas. Genie 2000 was chosen because of its capability of handling multiplet peaks and because of its automation of calculating peak areas. In a gamma-ray analysis software intercomparison by the IAEA [48], Genie 2000 was observed to produce good results for peak areas and uncertainties and thus justifies its use in this research. A total of 15 peaks were used in this analysis, and are displayed in Table 11. These data are provided from the Laboratoire National Henri Becquerel [49].

Table 11: Photons Used in Fission Product Analysis

\begin{tabular}{|c|c|c|}
\hline Nuclide & Photon Energy (keV) & Intensity (per 100 decays) \\
\hline${ }^{99 \mathrm{~m}} \mathrm{Tc} /{ }^{99} \mathrm{Mo}[50]$ & 140.511 & $89.6(17)^{1}$ \\
\hline \multirow{3}{*}{${ }^{99} \mathrm{Mo}[50]$} & 181.068 & $6.01(11)$ \\
\cline { 2 - 3 } & 739.500 & $12.12(15)$ \\
\cline { 2 - 3 } & 777.921 & $4.28(8)$ \\
\cline { 2 - 3 } & 162.6628 & $6.26(9)$ \\
\cline { 2 - 3 } & 304.872 & $4.30(4)$ \\
\cline { 2 - 3 } & 423.721 & $3.11(3)$ \\
\hline \multirow{3}{*}{${ }^{140} \mathrm{Ba}[50]$} & 537.303 & $24.39(22)$ \\
\cline { 2 - 3 } & 328.761 & $20.8(3)$ \\
\cline { 2 - 3 } & 487.022 & $46.1(4)$ \\
\cline { 2 - 3 } & 815.781 & $23.72(12)$ \\
\hline \multirow{3}{*}{$\mathrm{140} \mathrm{La}[50]$} & 1596.203 & $95.40(8)$ \\
\cline { 2 - 3 } & 724.193 & $54.27(22)$ \\
\hline \multirow{2}{*}{${ }^{95} \mathrm{Zr}[51]$} & 756.729 & $99.808(7)$ \\
\hline${ }^{95} \mathrm{Nb}[51]$ & 765.803 & \\
\hline
\end{tabular}

Each of these fission products decays by $\beta^{-}$emission (or internal transition in the case of ${ }^{99 \mathrm{~m}} \mathrm{Tc}$ ), so there is no photon emission inherent in the decay process but some are subject to gamma-gamma coincidences or gamma-X coincidences (when one of the gamma-rays is

\footnotetext{
${ }^{1}$ This intensity assumes equilibrium has been reached between ${ }^{99 \mathrm{~m}} \mathrm{Tc}$ and ${ }^{99} \mathrm{Mo}$
} 
internally converted). Therefore, the TCS factors need to be calculated for each of the affected gammas. The decay schemes for each of these nuclides can be found in Appendix 4.

\subsection{Activity Calculations}

The activity for each nuclide can be calculated individually for each photon emission that is observable in the spectrum. The activity of the nuclide is based on the peak area, the live time (and possibly the real time) of the count, the dead-time, the intensity of the gamma-ray emission, the efficiency of the counting mode for the energy of gamma-ray being observed, and the TCS factor. The method for calculating activity from gamma-ray peaks described in Semkow et al. [30] will be used and will be summarized here. This method uses the decay scheme of the nuclide being analyzed along with the peak and total efficiencies of the detector system. First, some definitions need to be produced that describe the decay pattern of the nuclide being analyzed. The row vector feeding of the different energy levels from the parent nuclide can be represented by:

$$
\boldsymbol{f}=\left(f_{0} f_{1} \ldots f_{n}\right)
$$

where:

$n$ is the number of excited levels in the daughter nucleus

$f_{i}$ is the fraction of decays that feed directly into the $i^{\text {th }}$ level (the $0^{\text {th }}$ level is the ground state)

The branching fractions (gamma transitions plus internal conversions) between levels can be represented by:

$$
\boldsymbol{x}=\left[\begin{array}{cccccc}
0 & 0 & 0 & \ldots & 0 & 0 \\
x_{10} & 0 & 0 & \ldots & 0 & 0 \\
x_{20} & x_{21} & 0 & \ldots & 0 & 0 \\
\vdots & \vdots & \vdots & \ddots & 0 & 0 \\
x_{n-10} & x_{n-11} & x_{n-12} & \ldots & \vdots & \vdots \\
x_{n 0} & x_{n 1} & x_{n 2} & \ldots & x_{n n-1} & 0
\end{array}\right]
$$

where $x_{i j}$ corresponds to the fraction of branches originating from level $i$ that feed into level $j$. This property makes $\mathbf{x}$ a lower triangular matrix because a level can only feed levels lower than itself. Both $\mathbf{f}$ and $\mathbf{x}$ represent probabilities of branching, and because of this, $\mathbf{f}$ and $\mathbf{x}$ must satisfy the following conditions:

$$
\sum_{i=0}^{n} f_{i}=1
$$




$$
\sum_{j=0}^{i-1} x_{i j}=1, \quad i=1 \ldots n
$$

Four more matrices should be constructed - c, a, e, and $\mathbf{b}$ - and are functions of $x_{i j}$, the peak efficiencies for the counting mode $\varepsilon_{P i, j}$, the total efficiencies for the counting mode $\varepsilon_{T i, j}$, and the internal conversion coefficients $\alpha_{i j}$. The e matrix has been modified from the original work to incorporate coincidence summing involving $X$-rays that arise from internal conversion, so this matrix also depends on the average total efficiency $\varepsilon_{T X}$ of the $X$-rays emitted by the nuclide being analyzed. For this system, the only $\mathrm{X}$-rays considered will be those originating from the Kshell. These matrices are defined as follows:

$$
\begin{gathered}
c_{i j}=\frac{x_{i j}}{1+\alpha_{i j}} \\
a_{i j}=c_{i j} \varepsilon_{P i j} \\
e_{i j}=c_{i j} \varepsilon_{T i j}+c_{i j} \alpha_{i j} \varepsilon_{T X} \\
b_{i j}=x_{i j}-e_{i j}
\end{gathered}
$$

where $j>i=0, \ldots, n-1$. Special provisions must be taken for constructing the e matrix for Singles mode. For this particular counting mode, two events are summed together only if they occur in the same crystal. Therefore it is not appropriate to use the full total efficiency of Singles mode because this incorporates all eight crystals. To account for this, the total efficiency for Singles mode should be averaged over all eight crystals before being incorporated in the e matrix, which will account for TCS occurring in the same crystal. Likewise, the a matrix needs to be modified a bit for Singles mode. As explained below, the a matrix takes into account transitions involving full-energy depositions of the photon of interest as well as summing-in from cascading photons. In Singles mode, the peak efficiency should incorporate all crystals for single transition energy depositions, but should only incorporate summing-in that occurs in the same crystal. Therefore, an additional matrix should be constructed for Singles mode:

$$
a_{i j S}=c_{i j} \frac{\varepsilon_{P i j}}{8}
$$

From these matrices, one can create two other matrices, $\mathbf{A}$ and $\mathbf{B}$, which account for all possible decay paths and coincidences regardless of the actual number of transitions that occur in a single decay. They are defined as: 


$$
\begin{gathered}
\boldsymbol{A}=\sum_{k=1}^{n} \boldsymbol{a}^{k} \\
\boldsymbol{B}=\boldsymbol{E}+\sum_{k=1}^{n} \boldsymbol{b}^{k}
\end{gathered}
$$

where the $k^{\text {th }}$ power is calculated via matrix multiplication and $\boldsymbol{E}$ is the unit matrix. For Singles mode, a modification must be made to the $A$ matrix:

$$
\boldsymbol{A}_{S}=\boldsymbol{a}\left(\mathbf{1}+\sum_{k=1}^{n-1} \boldsymbol{a}_{\boldsymbol{S}}{ }^{\boldsymbol{k}}\right)
$$

Two more diagonal matrices must be defined as well:

$$
\begin{gathered}
\boldsymbol{N}=\operatorname{diag}\left([\boldsymbol{f} \boldsymbol{B}]_{i}\right) \\
\boldsymbol{M}=\operatorname{diag}\left(\boldsymbol{B}_{i 0}\right)
\end{gathered}
$$

where matrix multiplication is implied.

These matrices can each be interpreted in a practical way. The elements $c_{i j}$ can be interpreted as the probability that a transition originating from energy level $i$ will go to energy level $j$ in a single transition and emit a gamma-ray as opposed to being internally converted. The elements $a_{i j}$ can be interpreted as the probability that a transition originating from energy level $i$ will go to energy level $j$ in a single transition and emit a gamma-ray, with that gamma-ray being fully absorbed in the detector system. The elements $e_{i j}$ represent the probability that a transition originating from energy level $i$ will decay to energy level $j$ and deposit some energy in the detector system - whether that be a full energy deposition, a partial energy deposition, or energy depositions from X-rays. The elements $b_{i j}$ represent the probability of a detection-free transition to energy level $j$ originating from energy level $i$ (i.e. a single transition occurs from energy level $i$ to energy level $j$ without producing an event in the detector system). The elements $A_{i j}$ (or $A_{i j, S}$ for Singles mode) represent the probability that a series of transitions originating from energy level $i$ will go to energy level $j$ emitting gamma-rays at each transition and all gamma-rays will deposit their full energy in the detector system (any crystal in Add Back mode or the same crystal in Singles mode). This may represent full-energy depositions of single transitions or sum peaks from multiple transitions. The elements $B_{i j}$ represent the probability that a series of detection-free transitions originating from energy level $i$ will go to energy level $j$ (i.e. a nucleus at energy level $i$ will decay to energy level $j$ without producing any events in the 
detector system). In the case of Singles mode, $B_{i j}$ should be interpreted as the probability that a series of detection-free transitions from level $i$ to level $j$ occurs only for the crystal that ends up observing the main gamma of interest. For the purposes of this analysis, $B_{i i}$ (the probability of a detection-free transition that originates and ends up at the same level) is 1 . The elements $N_{i i}$ represent the probability that a parent nucleus will decay to the $i^{\text {th }}$ excited state of the daughter nucleus without producing any events in the detector system (or the crystal observing the main gamma-ray for Singles mode). The elements $M_{i i}$ represent the probability that a daughter nucleus at energy level $i$ will decay to the ground state without producing any events in the detector system (or in the crystal observing the main gamma-ray for Singles mode).

To complete the analysis, the matrix $\mathbf{S}$ must be constructed with the following definition:

$$
S=N A M
$$

where matrix multiplication is implied. For Singles mode, $\mathbf{A}$ should be replaced with $\mathbf{A}_{\mathbf{s}}$. The above definition has been tweaked slightly from the original paper for the purposes of this analysis. In the above equation, $S_{i j}$ represents the probability that a decay from the parent nuclide will produce a count in the peak equivalent to the energy difference between level $i$ and level $j$ of the daughter nucleus, whether that count occurred from a single gamma-ray or from multiple gamma-rays. The $\mathbf{S}$ matrix takes into account summing-in, summing-out, the peakefficiency equation of the detector system, and the intensity of the gamma-ray in the nuclide's decay scheme. To calculate the activity, the dead-time corrected count rate in the spectrum should be divided by the appropriate element in the $\mathbf{S}$ matrix in order to obtain an estimation of the activity of the parent nuclide in the sample:

$$
A=\frac{R}{S_{i j}}
$$

where:

$A$ is the estimated activity of the nuclide in the sample at the time of the count $R$ is the dead-time corrected count rate of the full-energy peak being analyzed $S_{i j}$ is the element of the $\mathbf{S}$ matrix that corresponds to the peak being analyzed 
This activity estimation is valid if the actual count time of the sample is short compared to the effective half-life of the nuclide being analyzed. To correct the observed peak area for deadtime, the following equation should be used:

$$
P_{C}=\frac{P_{U}}{F(E)}
$$

where:

$P_{U}$ is the uncorrected peak area

$F(E)$ is the fraction of events with energy $E$ that are not lost due to dead-time, as explained in Chapter 8.

If the nuclide being analyzed has a short half-life compared with the count time, then decay during counting needs to be taken into consideration. The number of decays that occur over a time $d t$ can be represented by [4]:

$$
d D=A(t) d t
$$

where:

$d D$ is the number of decays that occur

$A(t)$ is the activity of the nuclide at time $t$

Integrating over the whole count time $T$, Equation (9.18) becomes:

$$
D=\int_{t=0}^{T} A(t) d t
$$

where $D$ is now the total number of decays occurring over the count time $T$. It should be noted that $T$ represents the real time, not simply the Live Time of the count. The activity can be written out as [4]:

$$
A(t)=A_{0} e^{-\lambda t}
$$

where:

$A_{0}$ is the activity at $t=0$

$\lambda$ is the decay constant of the nuclide

Substituting Equation (9.20) into Equation (9.19): 


$$
D=\int_{t=0}^{T} A_{0} e^{-\lambda t} d t
$$

Integrating Equation (9.21) over $t=0$ to $t=T$ :

$$
D=\frac{A_{0}}{\lambda}\left(1-e^{-\lambda T}\right)
$$

The matrix $\mathbf{S}$ in Equation (9.15) gives the number of counts observed in a given peak per decay of the nuclide. The number of counts observed in a given peak is therefore related to the number of decays over the count time by:

$$
N_{i j}=S_{i j} D
$$

where:

$N_{i j}$ is the number of counts observed in the peak corresponding to a transition in the nuclide from energy level $i$ to energy level $j$

$S_{i j}$ is element $(i, j)$ from the matrix $\mathbf{S}$ for the nuclide of interest

Equation (9.23) can be substituted into Equation (9.22) to relate the activity at the start of counting to the number of counts observed in the spectrum:

$$
A_{0}=\frac{N_{i j}}{S_{i j}} \frac{\lambda}{\left(1-e^{-\lambda T}\right)}
$$

Note that $N_{i j}$ must be corrected for Dead-Time using the methods described in Chapter 8 [52].

\subsection{Uncertainty in Activity Calculations}

There is inherent uncertainty in all terms used in the activity calculations. This stems from a variety of sources including the random nature of radioactive decay, background radioactivity fluctuations, system noise, sample geometry, sample placement within the detector system, the random nature of physical interactions between radiation and the detector system, the random nature of the detector response, uncertainties in the decay scheme, and environmental fluctuations (e.g. temperature, barometric pressure, humidity, etc...). The uncertainties for the direct feeding of each energy level $\left(f_{i}\right)$, transitions between energy levels $\left(x_{i j}\right)$, and internal conversion coefficients $\left(\alpha_{i j}\right)$ are taken from the decay scheme. The $\varepsilon_{P}(E)$ uncertainty is obtained from the variance-covariance matrix calculated in Equation (7.41). The uncertainty in $\varepsilon_{T}(E)$ is calculated from the uncertainty in $\varepsilon_{P}(E)$ and the uncertainty in $P / T(E)$. The uncertainty in the counting live time is assumed to be negligible compared to the uncertainties 
in the other variables. One source of uncertainty is not considered in this research is the beta particles that are emitted from these nuclides. Beta particles that penetrate the aluminum endcap can contribute to the pulse in the detector [4]. Beta particles can also produce bremsstrahlung radiation in the aluminum end-cap and surround materials. All of these sources of radiation can contribute to the continuum in the spectrum and can cause summing-out of relevant peaks [4].

When multiplying matrices together, special consideration must be made in calculating the uncertainty in each term. The uncertainty of a matrix $\mathbf{A}$ is contained in the matrix dA. The element $d A_{i j}$ contains the uncertainty in the element $A_{i j}$. Consider the matrix $\mathbf{G}$ such that:

$$
G=A B
$$

where $\mathbf{A}$ and $\mathbf{B}$ are independent. For a given function $f(x, y)$, the uncertainty in the function can be described by [53]:

$$
d f(x, y)=f(x+d x, y+d y)-f(x, y)
$$

From Equation (9.26), the uncertainty dG is:

$$
\begin{gathered}
d G=(A+d A)(B+d B)-A B \\
d G=A B+A(d B)+(d A) B+(d A)(d B)-A B \\
d G=A B+A(d B)+(d A) B+(d A)(d B)-A B \\
d G=A(d B)+(d A) B+(d A)(d B)
\end{gathered}
$$

The term $(\mathbf{d A})(\mathbf{d B})$ is considered negligible, so $\mathbf{d G}$ becomes:

$$
d G=A(d B)+(d A) B
$$

The uncertainty in each term for the activity calculation has been propagated through all steps in order to obtain the uncertainty in the final activity calculation from Equation (9.16). The uncertainty in all terms represents one standard deviation. 


\section{Chapter 10: Analyzing Uranium Samples for Activity over Time}

Parts of the original samples were radiochemically separated and beta counted to determine the original nuclide content of the samples at the respective zero time (i.e. the endof-bombardment time). The three parents in the three pairs being analyzed were calculated for original concentration using this method in both samples. The original concentrations of these nuclides are shown in Table 12.

Table 12: Initial activity concentrations of parent nuclides as determined by separated LANL

beta counting

\begin{tabular}{|c|c|c|}
\hline Sample & Nuclide & Concentration (atoms/g) \\
\hline \multirow{3}{*}{4126} & ${ }^{99} \mathrm{Mo}$ & $7.129 \mathrm{e} 10 \pm 0.4 \%$ \\
\cline { 2 - 3 } & ${ }^{140} \mathrm{Ba}$ & $7.026 \mathrm{e} 10 \pm 1.0 \%$ \\
\cline { 2 - 3 } & ${ }^{95} \mathrm{Zr}$ & $7.641 \mathrm{e} 10 \pm 1.8 \%$ \\
\hline \multirow{3}{*}{4130} & ${ }^{99} \mathrm{Mo}$ & $7.059 \mathrm{e} 10 \pm 0.5 \%$ \\
\cline { 2 - 3 } & ${ }^{140} \mathrm{Ba}$ & $6.944 \mathrm{e} 10 \pm 0.5 \%$ \\
\cline { 2 - 3 } & ${ }^{95} \mathrm{Zr}$ & $7.276 \mathrm{e} 10 \pm 1.5 \%$ \\
\hline
\end{tabular}

The activity for each of these nuclides (and their daughters) is calculated and displayed in Figures 35-106 along with the activity estimations provided by gamma-ray counting with the clover system. This is done in order to compare the analysis to a well-known and verified method of activity determination. Using Equation (9.16), the activity for each nuclide was estimated for each gamma-ray emission that was observable in each spectrum. This analysis was performed in both Add Back and Singles mode, as well as gamma-gamma coincidence mode when applicable. The decay curve estimations and activity residuals are displayed in Figures 36 $-107$.

A small amount of the daughter nuclides is expected to be present from parent decay during irradiation and a less significant portion from direct fission yield. In order to calculate the ingrowth of the daughter nuclide from parent decay, the activity of the parent must be calculated at time $t$ during irradiation. The equation for parent activity during irradiation is: 


$$
A_{P}(t)=\alpha\left(1-e^{-\lambda_{P} t}\right)
$$

where:

$\alpha$ is the saturation activity

$\lambda_{P}$ is the decay constant of the parent nuclide

$t$ is the time of irradiation

At $t=12,000$ seconds, the activity of each parent is known from beta counting. Equation (10.1) then becomes:

$$
A_{P}(t)=\frac{A_{P}(t=12,000)}{\left(1-e^{-\lambda_{P} * 12000}\right)}\left(1-e^{-\lambda_{P} t}\right)
$$

The activity of the daughter nuclide can be solved at time $t$ using a modification of the Bateman equation [54]:

$$
\frac{d N_{D}}{d t}=r A_{P}(t)-\lambda_{D} N_{D}(t)
$$

where:

$N_{D}(t)$ is the concentration of the daughter nuclide at time $t$

$r$ is the fraction of parent decays that go to the daughter

$A_{P}(t)$ is the activity of the parent at time $t$

$\lambda_{D}$ is the decay constant of the daughter nuclide

Using Equation (10.2) for $A_{P}(t)$ and integrating Equation (10.3) for $N_{D}(t)$, the daughter activity at time $t$ after the start of irradiation becomes:

$$
A_{D}(t)=\frac{A_{P}(t=12,000)}{\left(1-e^{-\lambda_{P} * 12000}\right)} r\left(1-e^{-\lambda_{D} t}-\frac{\lambda_{D}}{\lambda_{D}-\lambda_{P}}\left(e^{-\lambda_{P} t}-e^{-\lambda_{D} t}\right)\right)
$$

where $\lambda_{p}$ is the decay constant of the parent nuclide. Using this method, the activity of each of the daughter nuclides from the decay of the parents is given in Table 13. 
Table 13: Initial activity estimates of daughters from parent decay during irradiation

\begin{tabular}{|c|c|c|}
\hline \multirow{2}{*}{ Sample } & Daughter Nuclide & $\begin{array}{c}\text { Activity at Zero Time from } \\
\text { Parent Decay (Bq) }\end{array}$ \\
\hline \multirow{3}{*}{4126} & ${ }^{99 \mathrm{~m}} \mathrm{Tc}$ & $1024 \pm 16$ \\
\cline { 2 - 3 } & ${ }^{140} \mathrm{La}$ & $40.8 \pm 0.9$ \\
\cline { 2 - 3 } & ${ }^{95} \mathrm{Nb}$ & $0.43 \pm 0.01$ \\
\hline \multirow{3}{*}{4130} & ${ }^{99 \mathrm{~m}} \mathrm{Tc}$ & $983 \pm 15$ \\
\cline { 2 - 3 } & ${ }^{140} \mathrm{La}$ & $39.3 \pm 0.8$ \\
\cline { 2 - 3 } & ${ }^{95} \mathrm{Nb}$ & $0.40 \pm 0.01$ \\
\hline
\end{tabular}

There is also a small amount of activity present from direct fission yield. This can be estimated from the amount of parent nuclide present at the end of irradiation and using a yield ratio. The observed concentration of the parent is approximately proportional to the fission chain yield for the parent. Therefore, the equation for the concentration of the daughter nuclide from direct fission can be expressed by:

$$
N_{D}=\frac{F Y_{\text {Direct,Daughter }}}{F Y_{\text {Chain,Parent }}} N_{P}
$$

where:

$N_{D}$ is the concentration of the daughter product from direct fission yield at the end of irradiation $N_{P}$ is the concentration of parent product from fission chain yield at the end of irradiation $F Y_{\text {Direct,Daughter }}$ is the direct fission yield to the daughter using thermal neutrons $F Y_{\text {Chain,Parent }}$ is fission chain yield to the parent using thermal neutrons

The thermal ${ }^{235} \mathrm{U}$ fission chain yields from the parents and the direct yields for the daughters are displayed in Table 14 [55].

Table 14: Thermal fission yields for parent and daughter nuclides

\begin{tabular}{|c|c|}
\hline Fission Product Pair & $\begin{array}{c}\text { Parent Chain Yield (Daughter Direct Yield) in } \\
\% \text { per fission }\end{array}$ \\
\hline${ }^{99} \mathrm{Mo} /{ }^{99 \mathrm{~m}} \mathrm{Tc}$ & $6.11 \mathrm{E}+00(2.89 \mathrm{E}-08)$ \\
\hline${ }^{140} \mathrm{Ba} /{ }^{140} \mathrm{La}$ & $6.21 \mathrm{E}+00(5.22 \mathrm{E}-03)$ \\
\hline${ }^{95} \mathrm{Zr} /{ }^{95} \mathrm{Nb}$ & $6.50 \mathrm{E}+00(1.06 \mathrm{E}-04)$ \\
\hline
\end{tabular}

Equation (10.5) was not corrected for decay during irradiation. This is a valid approximation in this research for ${ }^{99 m} \mathrm{Tc}$ because Table 14 shows that the ${ }^{99 \mathrm{~m}} \mathrm{Tc}$ direct fission yield is eight orders 
of magnitude below the parent fission chain yield. Therefore, the contribution of initial activity to ${ }^{99 \mathrm{~m}} \mathrm{Tc}$ is small. The approximation is valid for ${ }^{140} \mathrm{La}$ and ${ }^{95} \mathrm{Nb}$ because the half-lives of these nuclides are long compared to the irradiation time. Using Table 14 and Equation (9.21), the daughter concentration (and the daughter activity) from direct fission can be calculated. These activities are displayed in Table 15.

Table 15: Initial activity estimates of daughters from direct fission yield

\begin{tabular}{|c|c|c|}
\hline \multirow{2}{*}{ Sample } & Daughter Nuclide & $\begin{array}{c}\text { Activity at Zero Time from } \\
\text { Direct Fission (Bq) }\end{array}$ \\
\hline \multirow{3}{*}{4126} & ${ }^{99 \mathrm{~m}} \mathrm{Tc}$ & $(3.24 \pm 0.05) \mathrm{E}-05$ \\
\cline { 2 - 3 } & ${ }^{140} \mathrm{La}$ & $1.21 \pm 0.03$ \\
\cline { 2 - 3 } & ${ }^{95} \mathrm{Nb}$ & $(5.14 \pm 0.09) \mathrm{E}-03$ \\
\hline \multirow{3}{*}{4130} & ${ }^{99 \mathrm{~m}} \mathrm{Tc}$ & $1.17 \pm 0.05) \mathrm{E}-05$ \\
\cline { 2 - 3 } & ${ }^{140} \mathrm{La}$ & $(4.74 \pm 0.11) \mathrm{E}-03$ \\
\cline { 2 - 3 } & ${ }^{95} \mathrm{Nb}$ & \\
\cline { 2 - 3 }
\end{tabular}

Table 16 shows the estimated initial daughter activity both from parent decay during irradiation and from direct fission yield.

Table 16: Total initial activity estimates of daughters

\begin{tabular}{|c|c|c|}
\hline Sample & Daughter Nuclide & Activity at Zero Time (Bq) \\
\hline \multirow{3}{*}{4126} & ${ }^{99 \mathrm{~m}} \mathrm{Tc}$ & $1024 \pm 16$ \\
\cline { 2 - 3 } & ${ }^{140} \mathrm{La}$ & $42.1 \pm 0.03$ \\
\cline { 2 - 3 } & ${ }^{95} \mathrm{Nb}$ & $0.439 \pm 0.01$ \\
\hline \multirow{3}{*}{4130} & ${ }^{99 \mathrm{~m}} \mathrm{Tc}$ & $983 \pm 15$ \\
\cline { 2 - 3 } & ${ }^{140} \mathrm{La}$ & $40.4 \pm 0.8$ \\
\cline { 2 - 3 } & ${ }^{95} \mathrm{Nb}$ & $0.405 \pm 0.01$ \\
\hline
\end{tabular}

The values in Table 16 have been incorporated into the activity estimations from beta counting, as shown by the "Expected" curves in Figures 36 - 107. The "Expected" curves are generated from the initial activities of each nuclide and corrected for decay over time. The equation for activity decay is [4]:

$$
A(t)=A_{0} e^{-\lambda t}
$$

where:

$A_{0}$ is the activity determined by beta counting at time $t=0$

$\lambda$ is the decay constant of the nuclide

$t$ is the time since irradiation 
The residuals for observed measurements when compared to expected values are defined as [56]:

$$
R_{i}=y_{i}-\hat{y}_{i}
$$

where:

$y_{i}$ is the measured value for the $\mathrm{i}^{\text {th }}$ data point

$\hat{y}_{i}$ is the expected value for the $i^{\text {th }}$ data point

Using Equation (10.7), the residuals activity for the purposes of this research is defined as:

$$
R_{i}=A_{\gamma, i}-A_{\beta, i}
$$

where:

$A_{\gamma, i}$ is the activity as determined by the measurements with the clover system for point $i$

$A_{b, i}$ is the activity as determined by the beta counting measurements, correcting for decay

The uncertainty in this difference is defined by [4]:

$$
\sigma\left(R_{i}\right)=\sqrt{\sigma^{2}\left(A_{\gamma, i}\right)+\sigma^{2}\left(A_{\beta, i}\right)}
$$

where $\sigma(f)$ represents the uncertainty in $f$. To calculate a point in a z-score residuals plot, the general form of the equation is [56]:

$$
r_{i}=\frac{R_{i}}{\sigma\left(R_{i}\right)}
$$

Therefore, the points in the following residuals plots are calculated with the following equation:

$$
r_{i}=\frac{A_{\gamma, i}-A_{\beta, i}}{\sqrt{\sigma^{2}\left(A_{\gamma, i}\right)+\sigma^{2}\left(A_{\beta, i}\right)}}
$$

\subsection{Activity Calculation Results from Sample 4126}

This Section will provide the results of the activity calculations and point out the obvious properties of the Figures. Explanations and discussions of the results will be provided in Section 10.3. As seen in Figures $36-43$, the ${ }^{95} \mathrm{Zr}$ and ${ }^{95} \mathrm{Nb}$ align fairly well with the activity estimates obtained from beta counting. All photons being analyzed are singlet emissions by $\beta^{-}$decay, so 
there is no TCS involved in the detection of these photons. Both the Add Back and Singles modes give some data points that are below $-1 \sigma$, but are mostly in the $-1 \sigma$ to $1 \sigma$ range. 


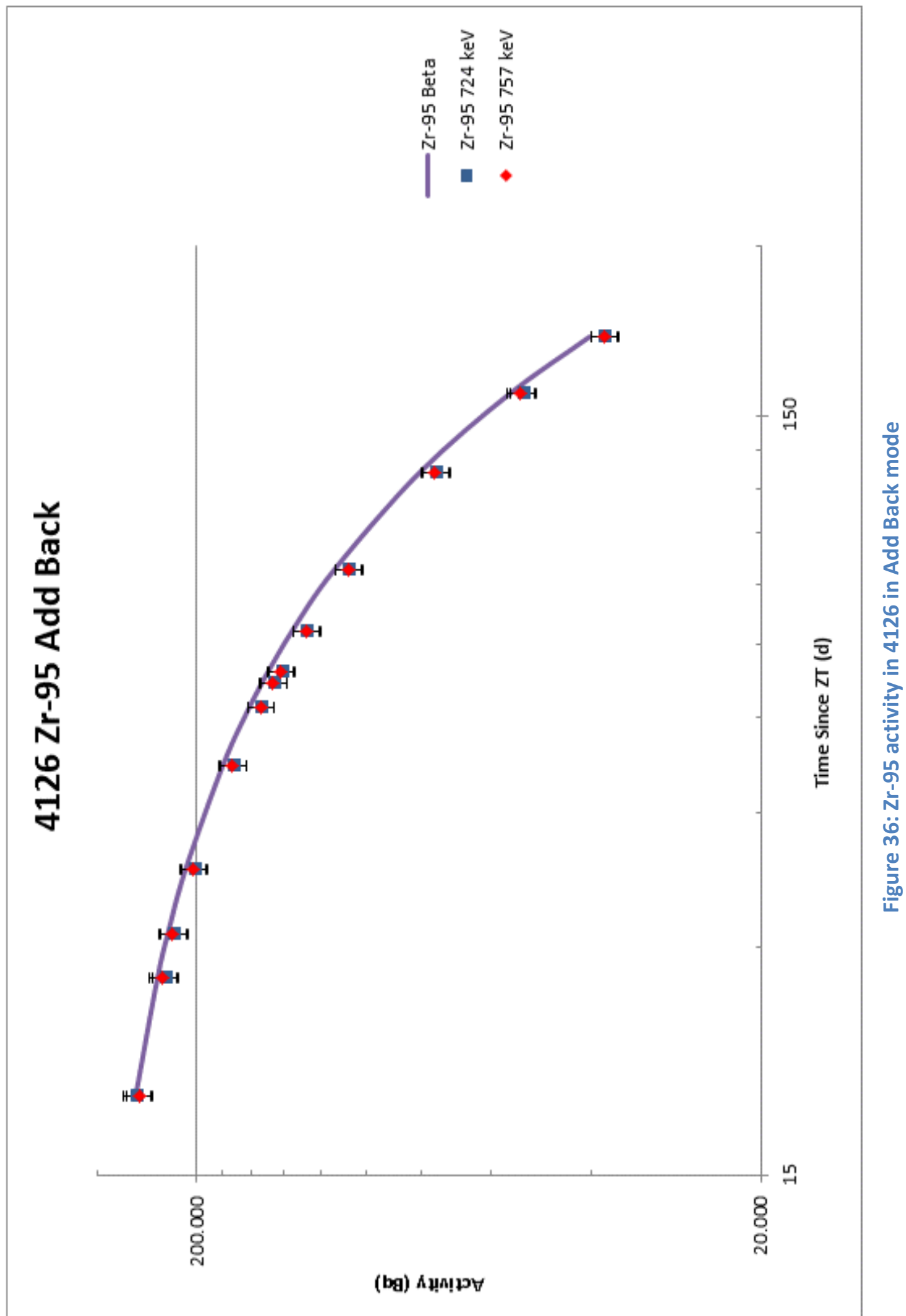




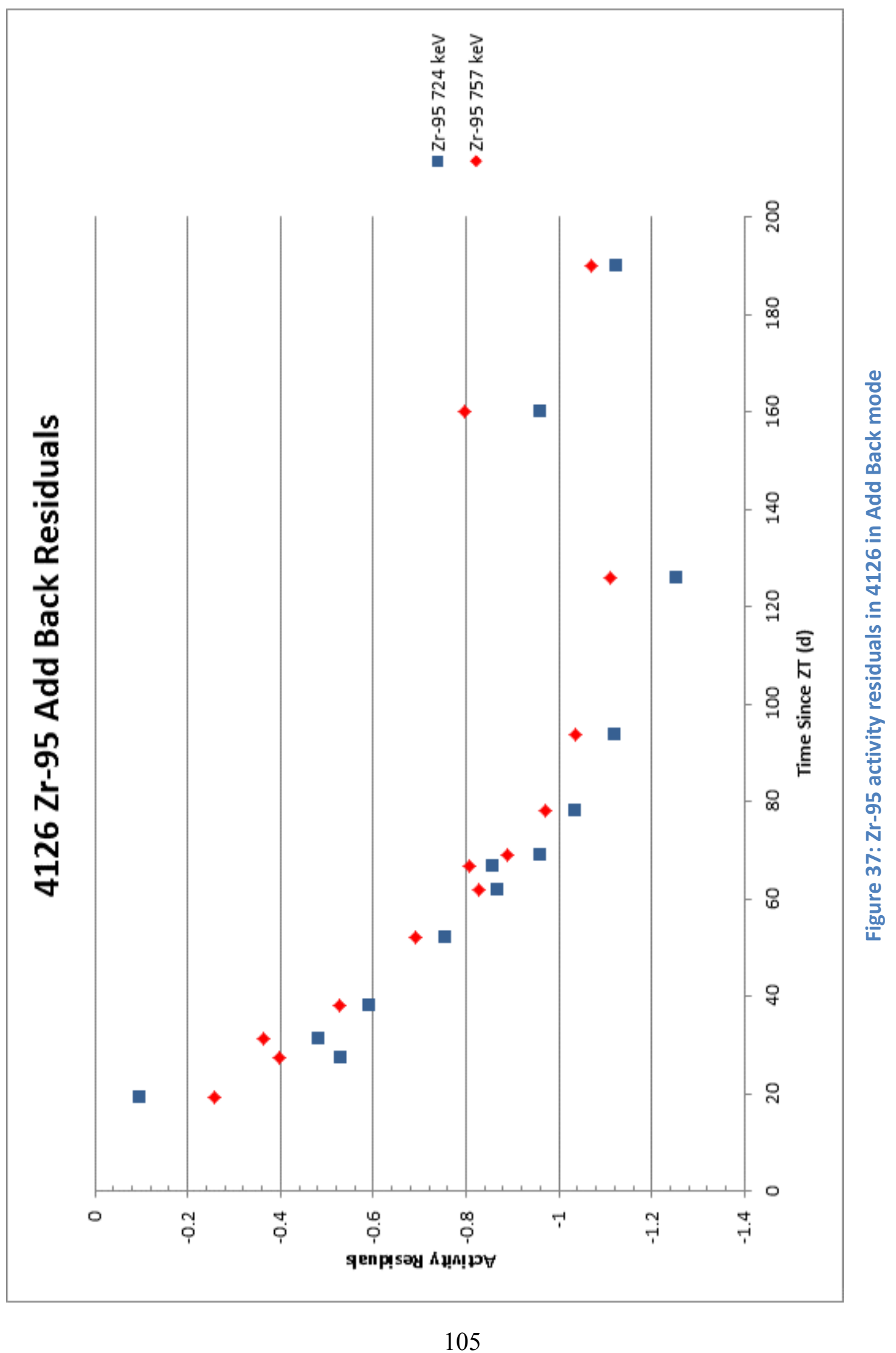




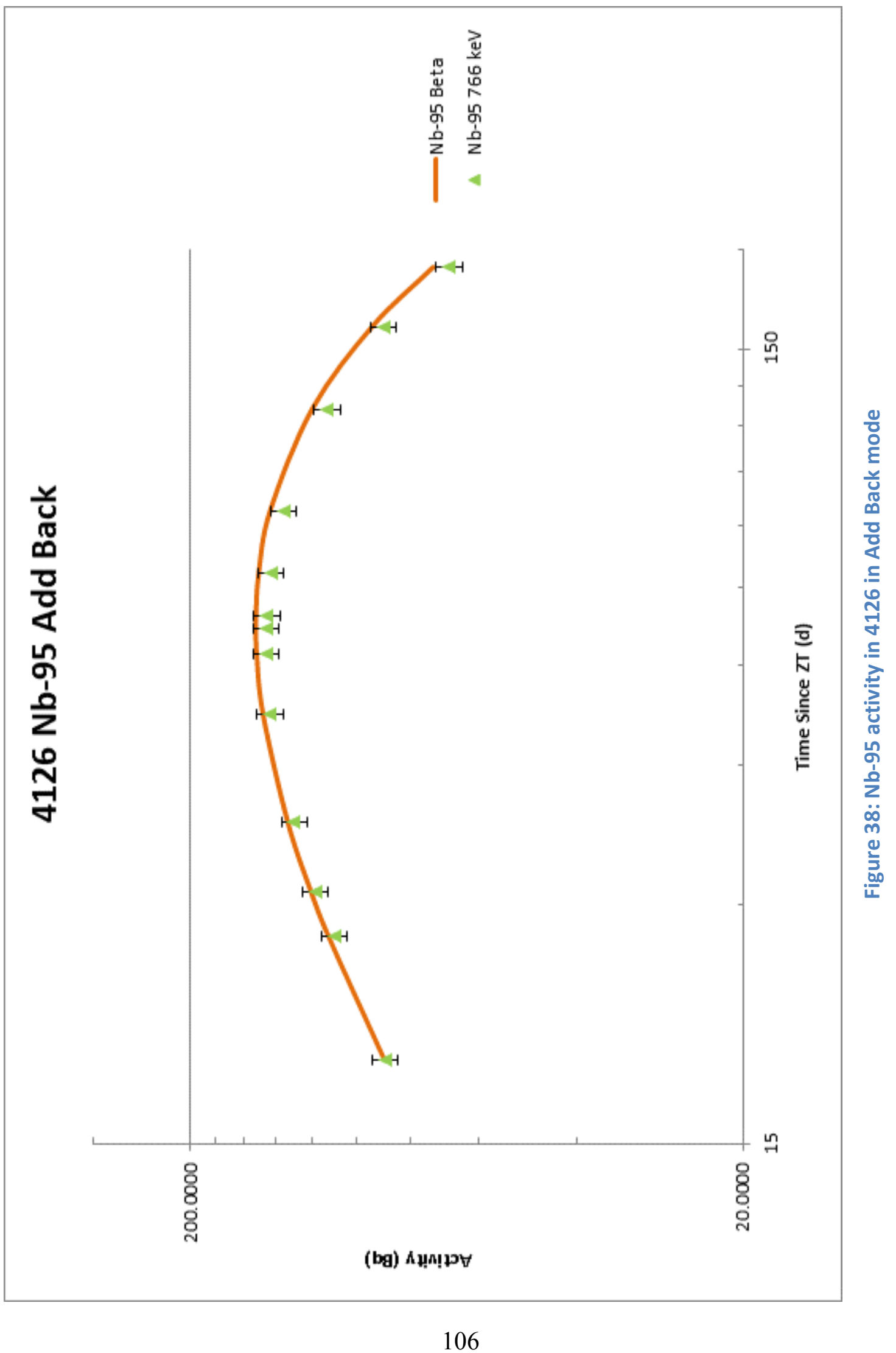




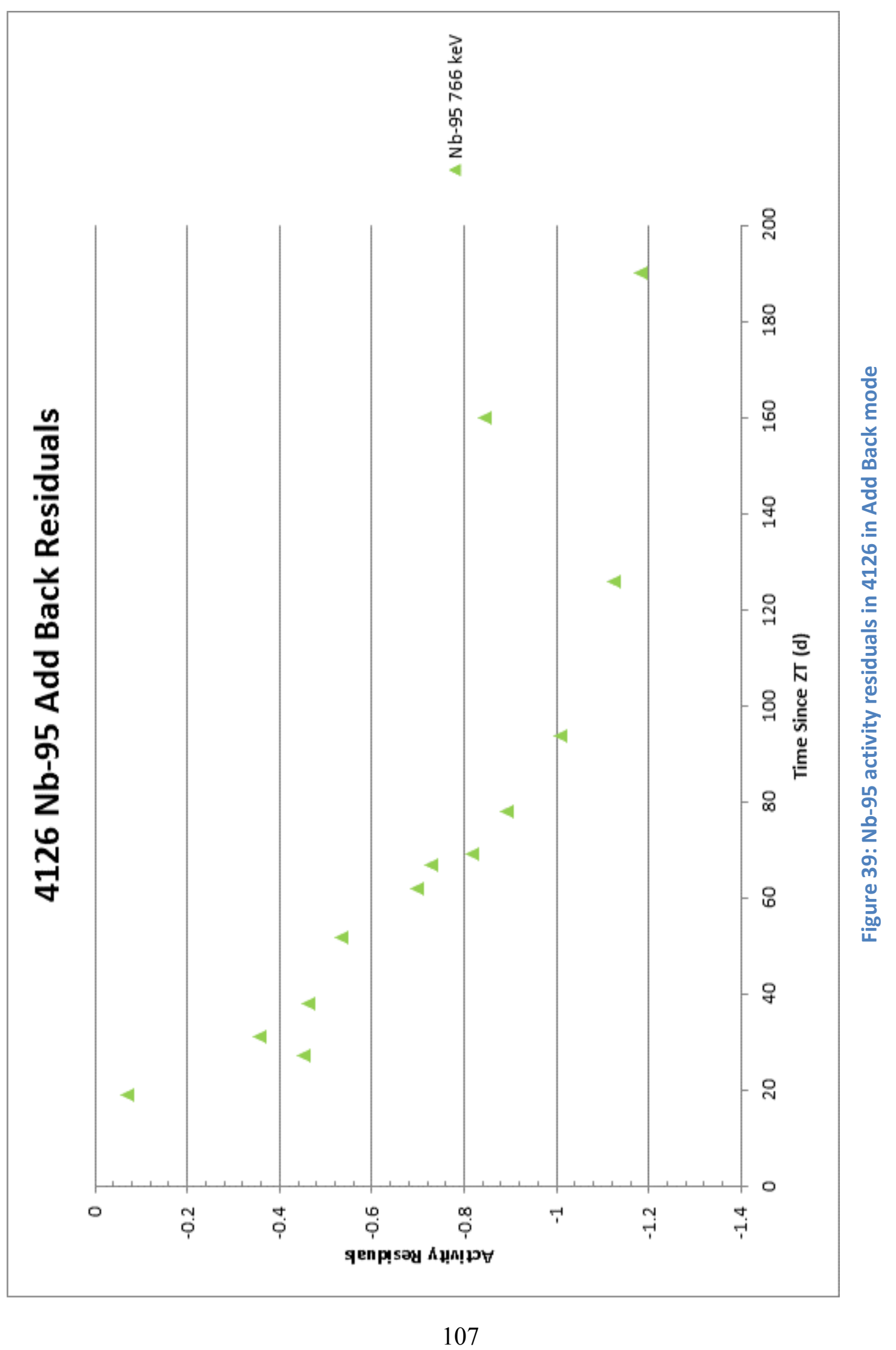




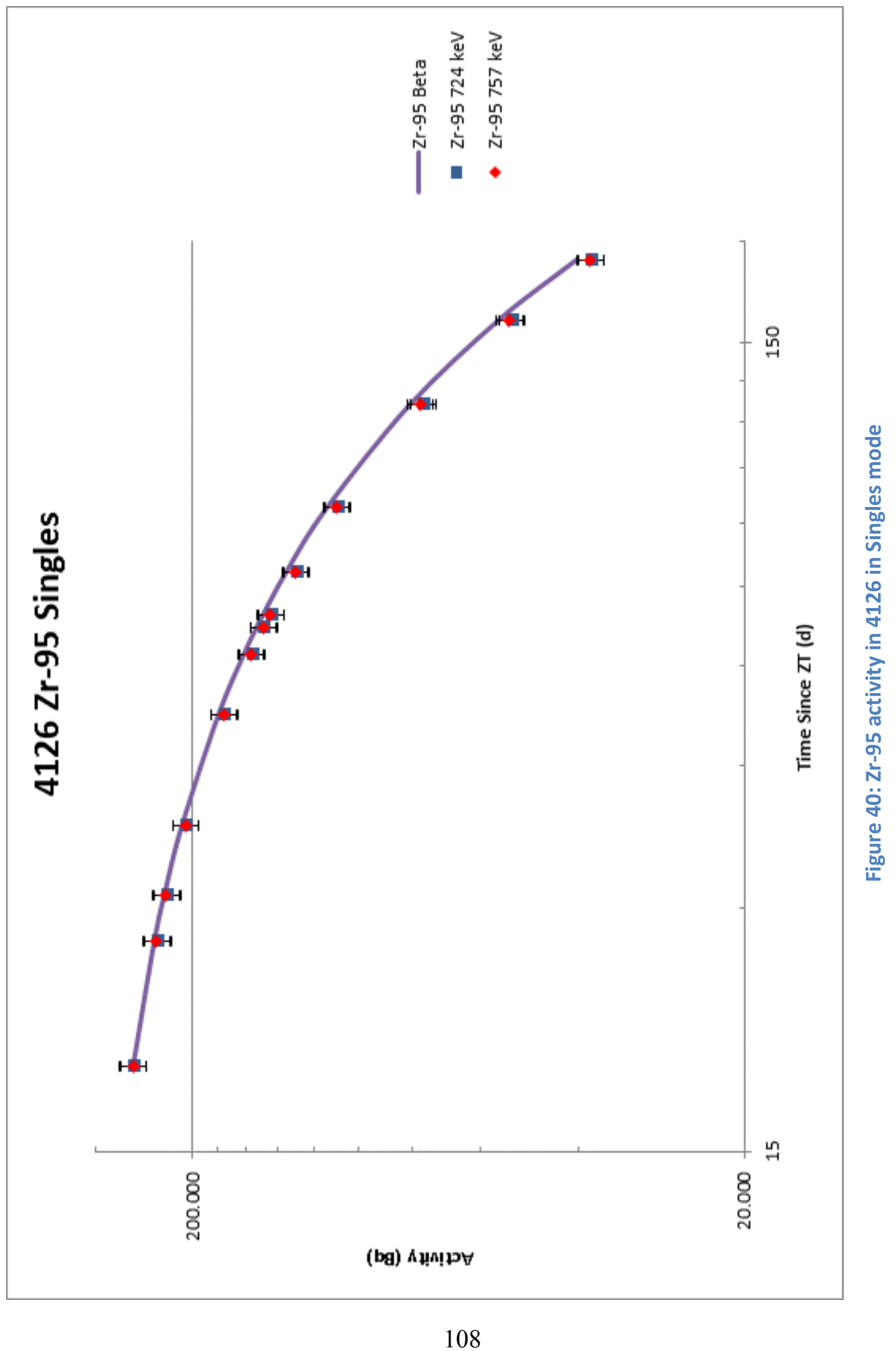




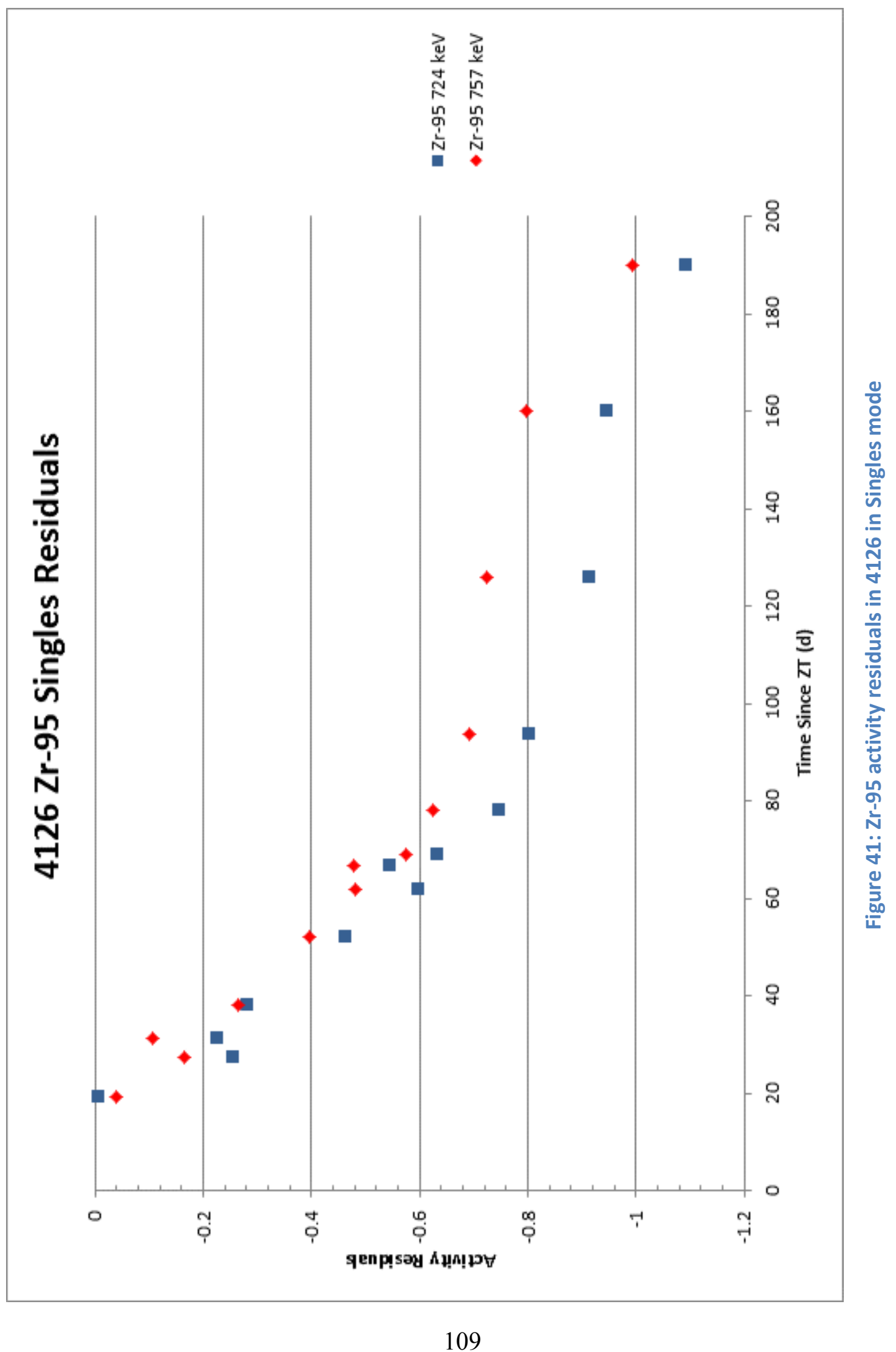




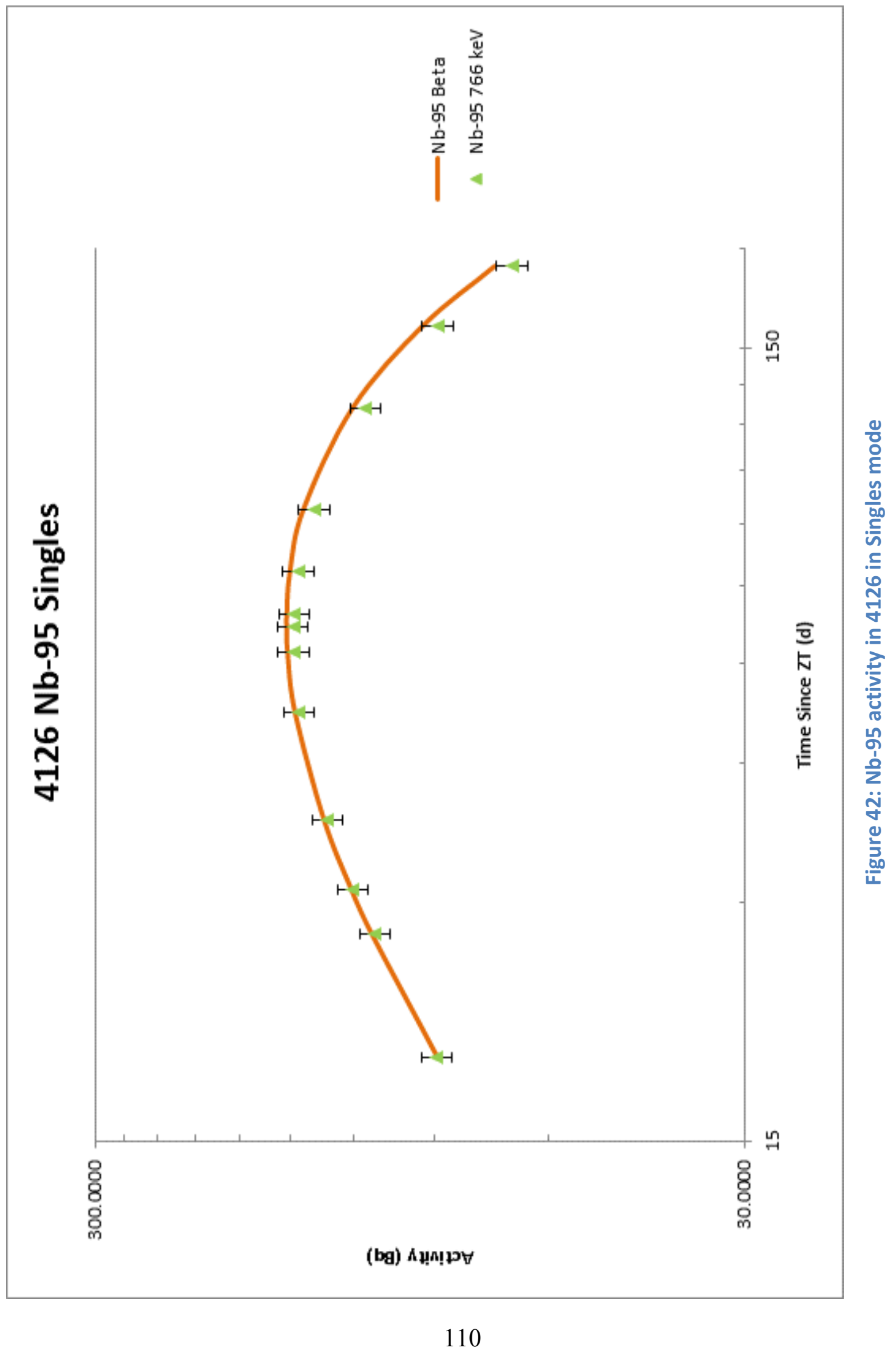




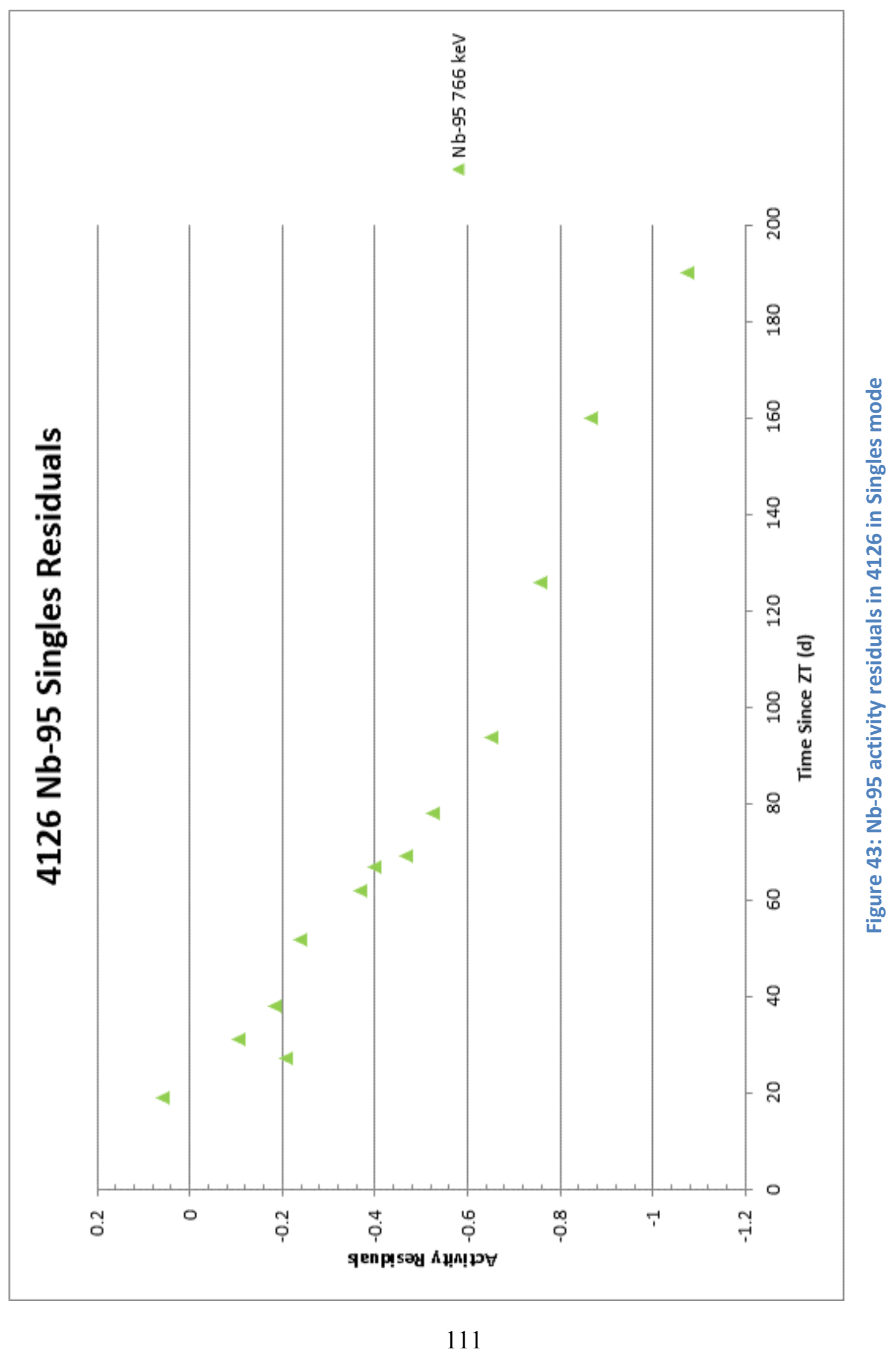


The activity estimations from ${ }^{140} \mathrm{Ba}$ and ${ }^{140}$ La give mixed results. Observing ${ }^{140} \mathrm{Ba}$, the decay scheme shows that the $163 \mathrm{keV}$ photon is heavily paired with the $305 \mathrm{keV}$ photon while the $423 \mathrm{keV}$ and $537 \mathrm{keV}$ photons are mainly singlet emissions. The $163 \mathrm{keV}$ photon also has a strong interference with the $163 \mathrm{keV}$ emission from ${ }^{235} \mathrm{U}$, though this does not significantly impact the analysis until the ${ }^{140} \mathrm{Ba}$ concentration has reached a low level. The half-life of ${ }^{235} \mathrm{U}$ is 704 million years, so the gamma-ray emission rate of the $163 \mathrm{keV}$ photon from ${ }^{235} \mathrm{U}$ will be essentially constant across all the spectra. The final spectrum of the 4126 sample taken showed a count rate of $0.0598 \pm 0.0040 \mathrm{cps}$ in Add Back mode and $0.0499 \pm 0.0050 \mathrm{cps}$ in Singles mode. These count rates were assumed to be the constant count rate due to the $163 \mathrm{keV}$ emission of ${ }^{235} \mathrm{U}$ because this was 189 days (about 15 half-lives) after the zero time of the sample, and so the ${ }^{140} \mathrm{Ba}$ contribution to this peak is assumed to be negligible. The calculated contribution from ${ }^{235} \mathrm{U}$ to the $163 \mathrm{keV}$ peak area was then subtracted from the peak area observed in each spectrum. As seen in Figure 44, the brown squares representing the activity calculations for the $163 \mathrm{keV}$ photon deviate from the expected line. This shows that some of the interference from ${ }^{235} \mathrm{U}$ is still contributing to this peak even after the correction.

As seen in Figure 45, in Add Back mode the $163 \mathrm{keV}$ activity residuals do fall within $-1 \sigma$ to $1 \sigma$ for almost all the data points, but activity calculations using the $305 \mathrm{keV}$ photon peak are consistently above $1 \sigma$. The singlet emissions of $423 \mathrm{keV}$ and $537 \mathrm{keV}$ show good agreement with the estimations from beta counting except possibly in the last spectrum where the peak areas were small. As seen in Figure 49 , in Singles mode all the ${ }^{140}$ Ba peaks give accurate estimates of the activity.

Observing ${ }^{140} \mathrm{La}$, the decay scheme shows that almost all emissions are part of cascading or coincident transitions. As shown in Figure 47, the activity calculations for Add Back mode using the $329 \mathrm{keV}$, the $487 \mathrm{keV}$, and the $1596 \mathrm{keV}$ residuals are consistently above $1 \sigma$. The 816 $\mathrm{keV}$ residuals are above the beta estimate, but are within $1 \sigma$. As shown in Figure 51 , all peaks are consistent with the beta counting activity estimates for Singles mode. 


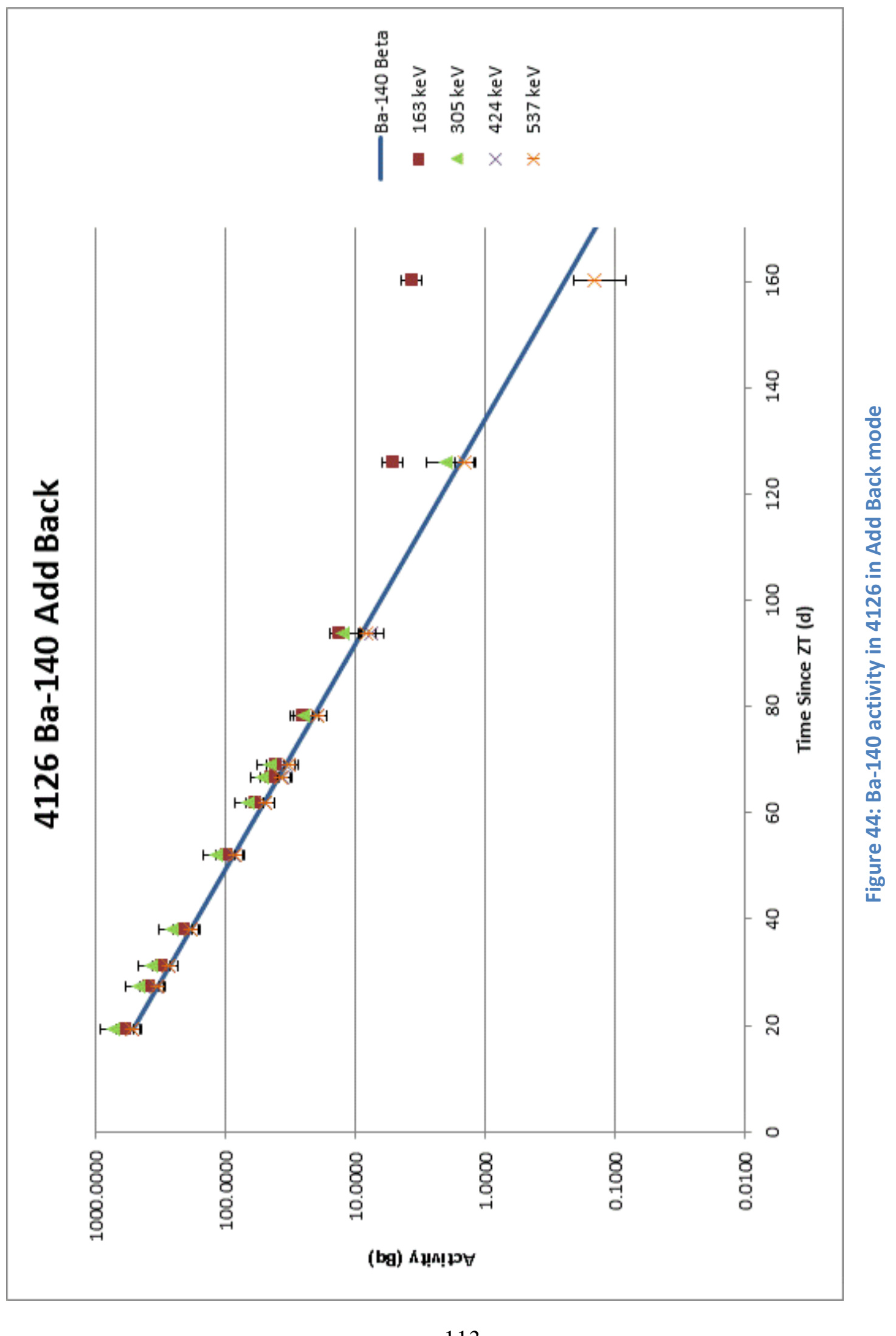




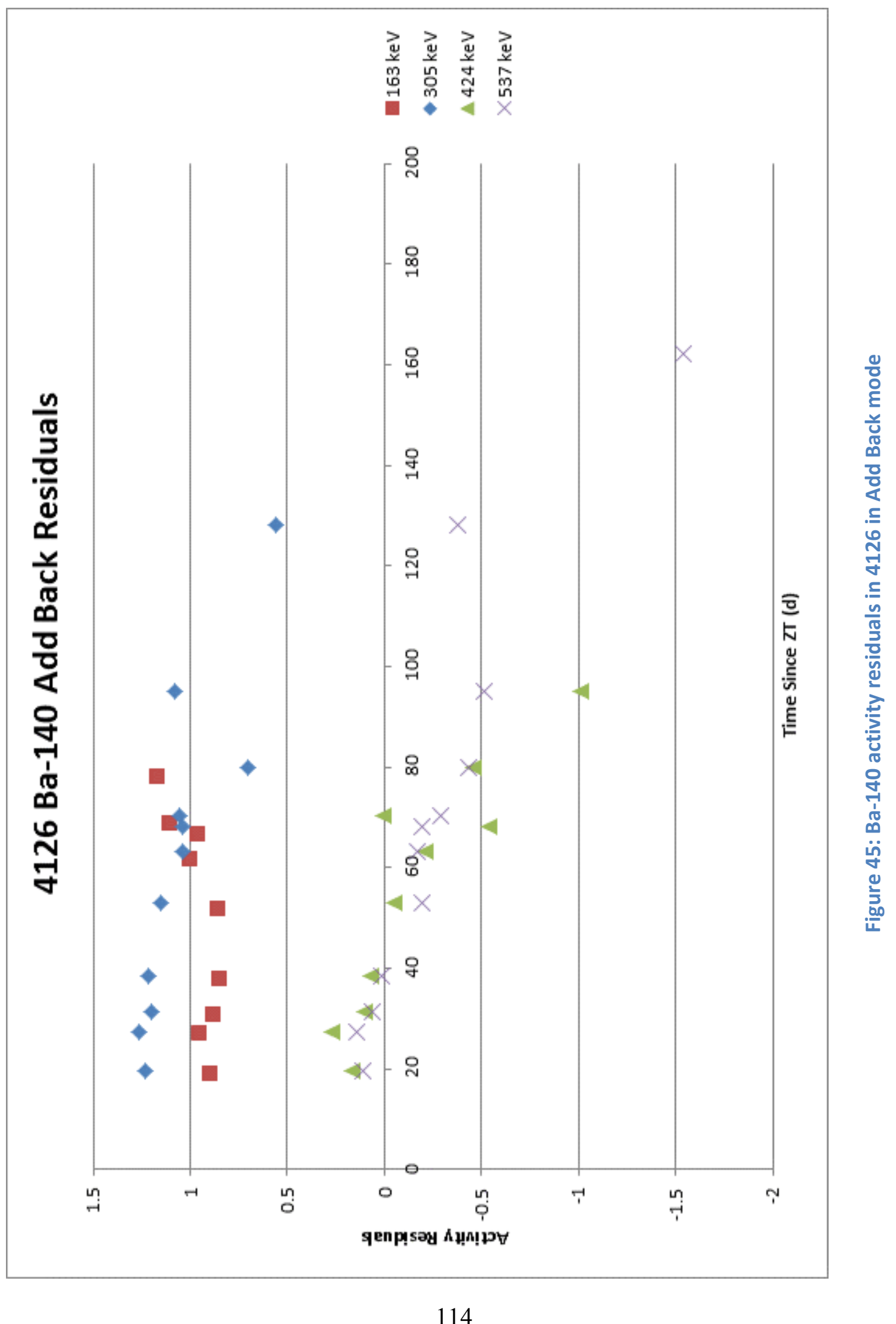




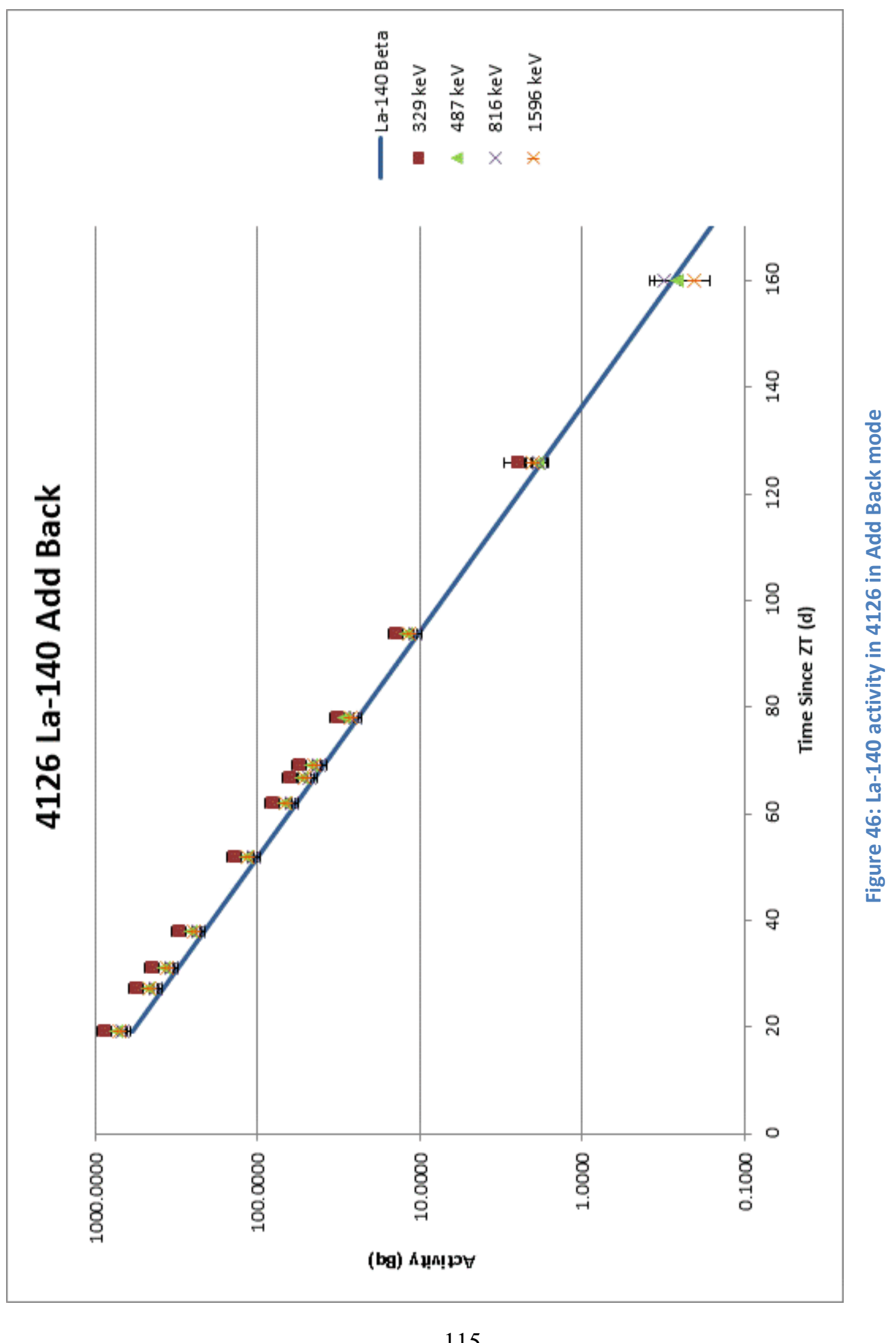




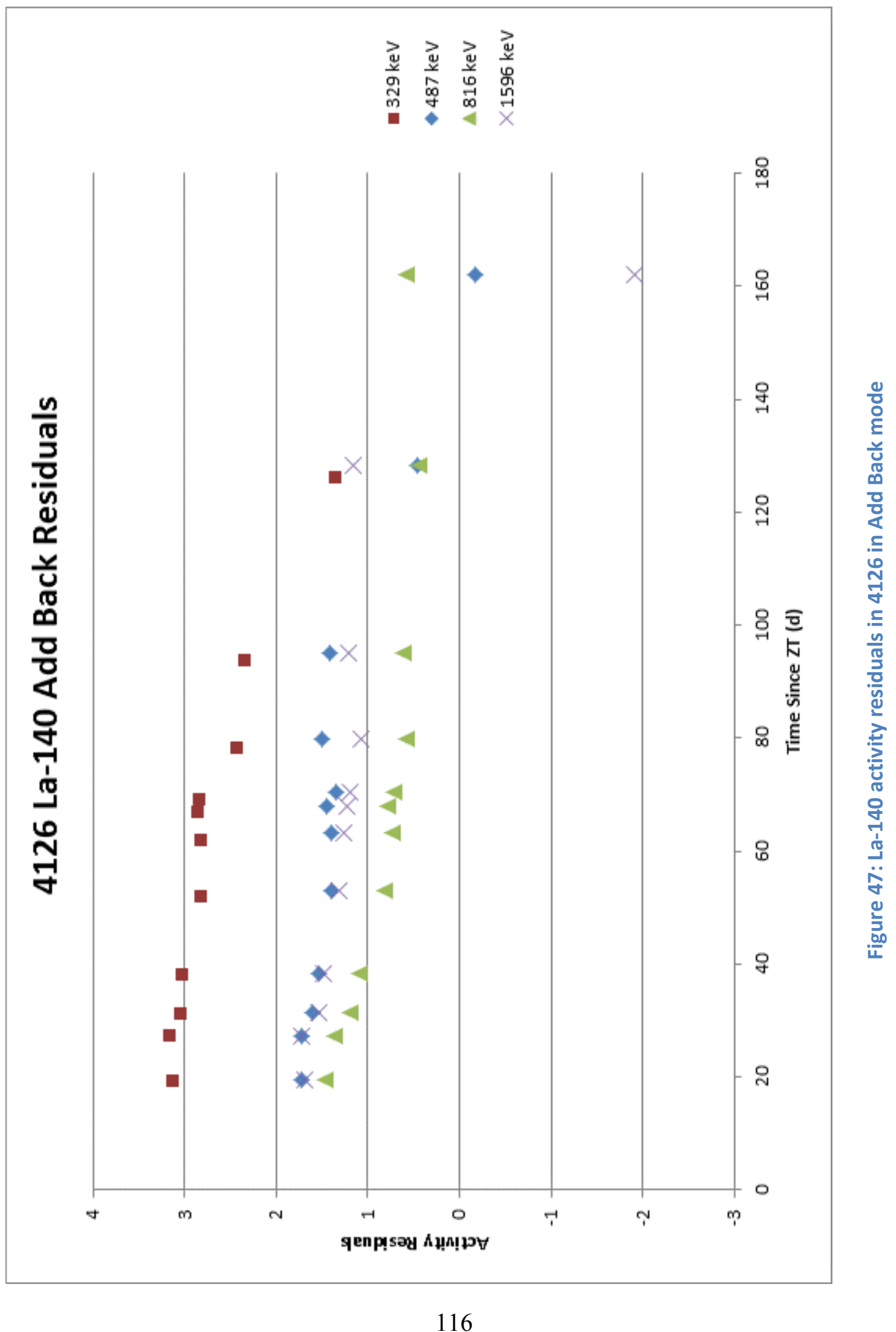




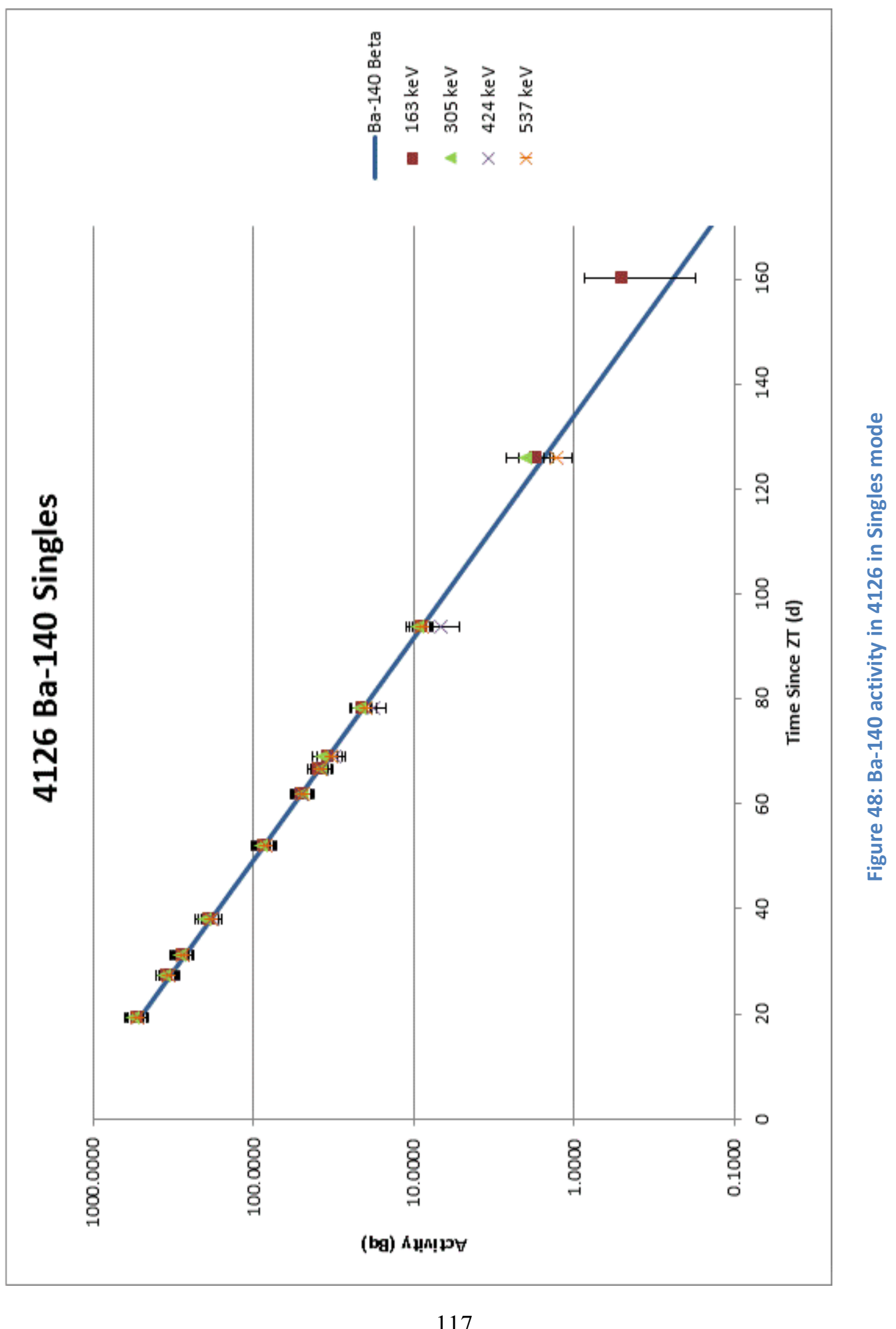




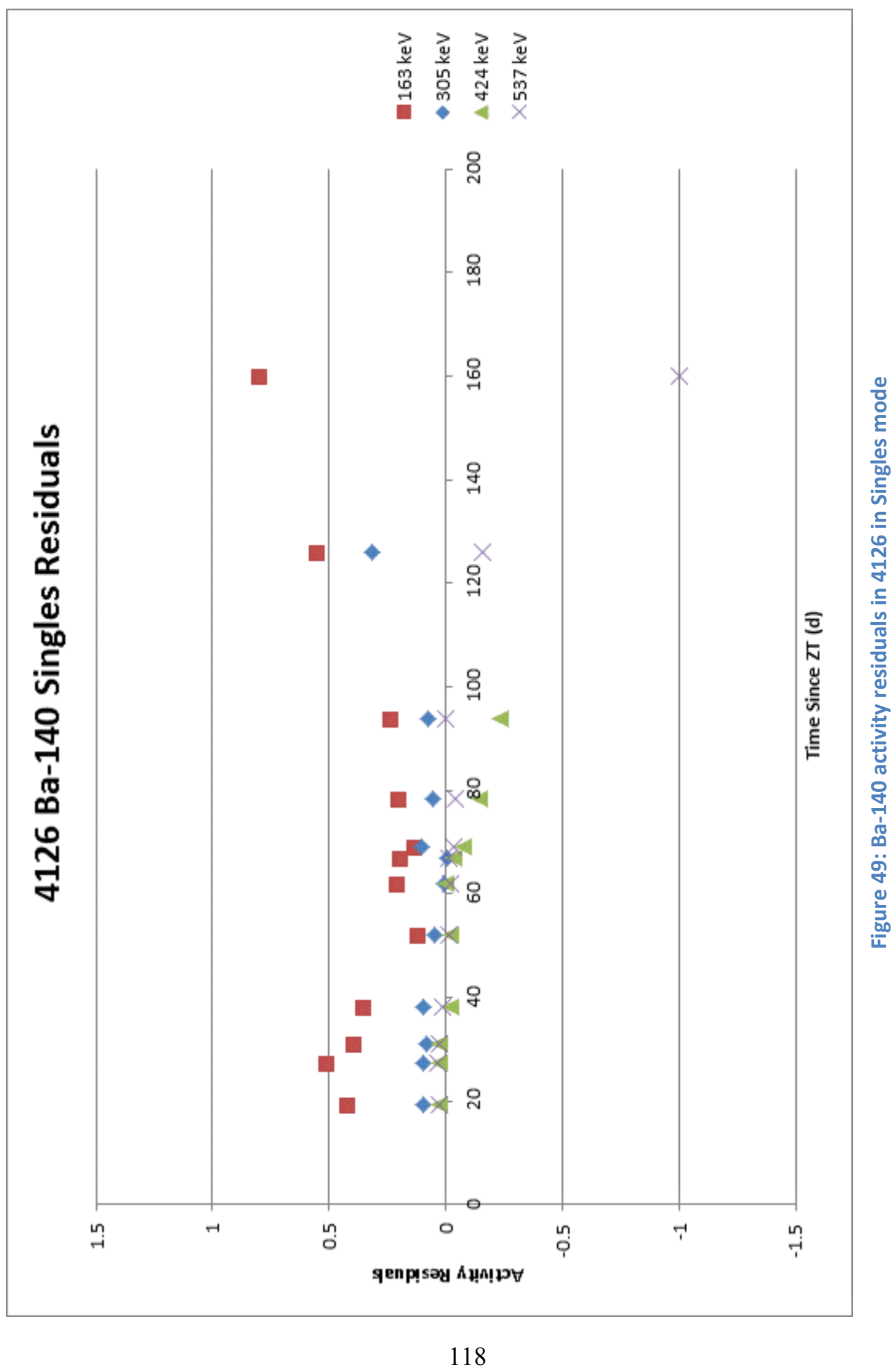




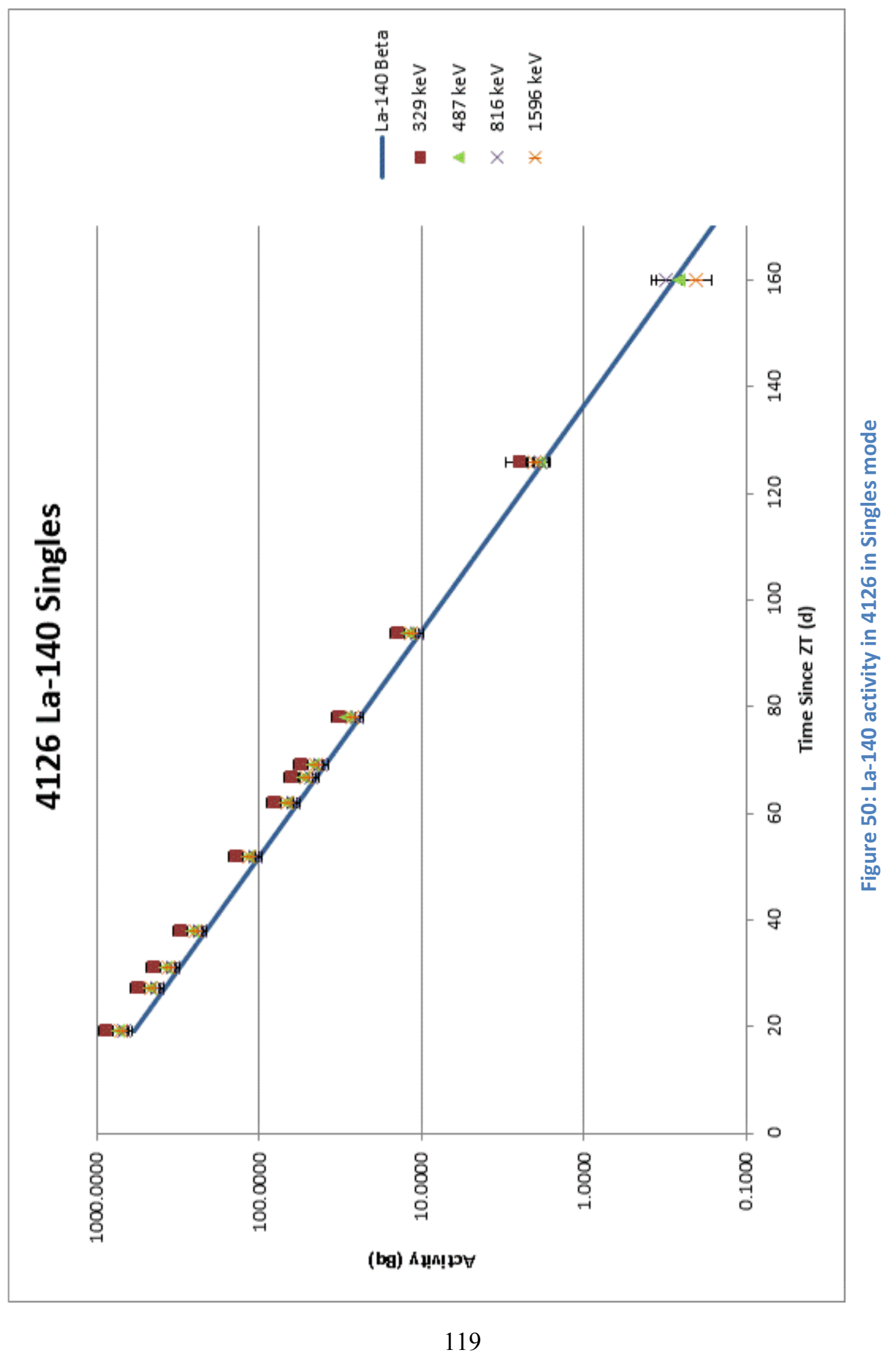




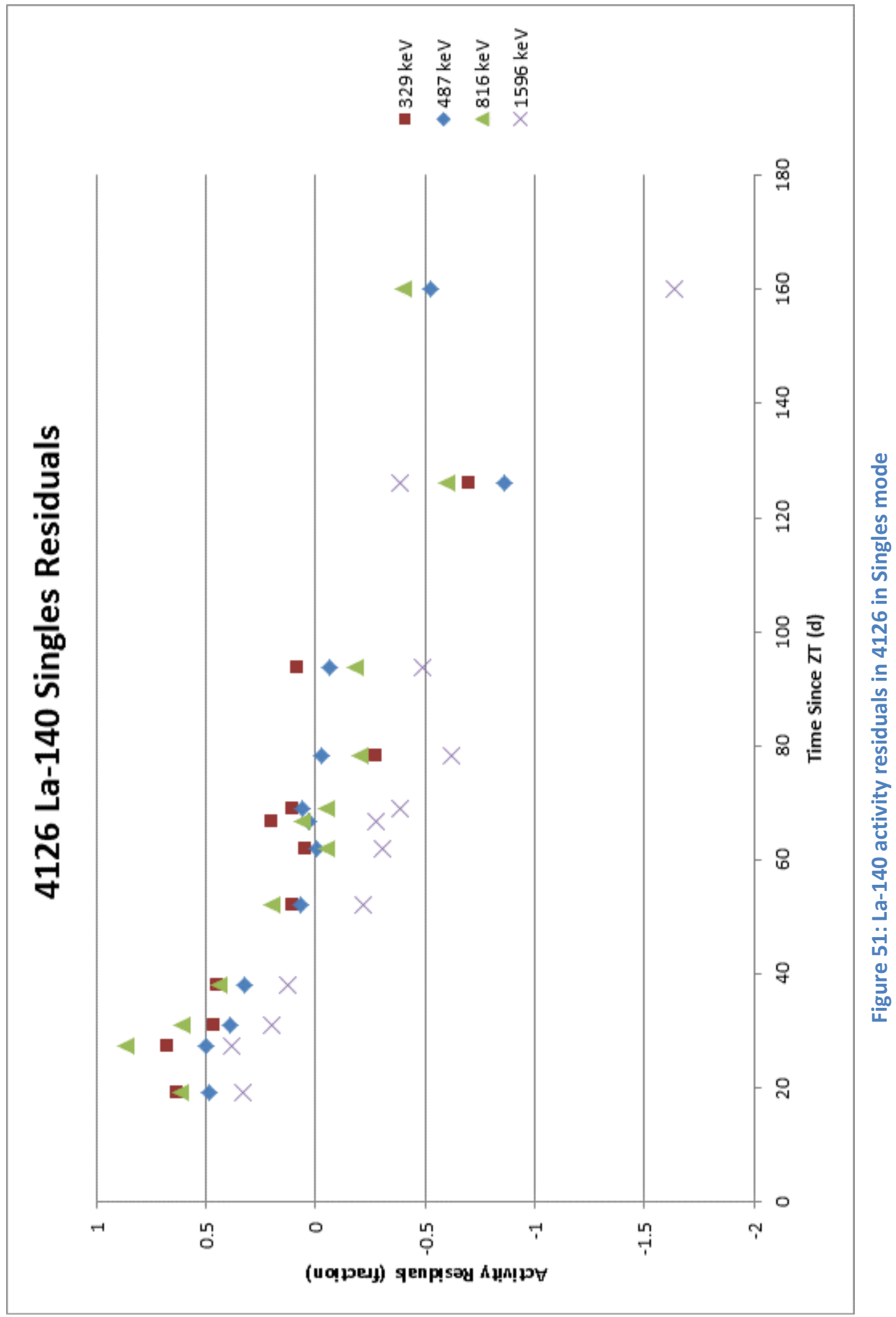


Because the first count on the 4126 sample did not occur until 19 days after the sample was removed from the reactor, most of the ${ }^{99} \mathrm{Mo} /{ }^{99 \mathrm{~m}} \mathrm{Tc}$ pair was expected to have decayed away. The first few spectra did show some activity from these nuclides. As shown in Figure 55, all the peaks in Add Back mode seemed to overestimate the activity when compared to beta counting, although the $778 \mathrm{keV}$ peak did agree with the beta counting estimates to within the error bars. The $141 \mathrm{keV}$ peak seemed to give mixed results for the ${ }^{99 \mathrm{~m}} \mathrm{Tc}$ activity estimates. Many of these peaks have low counts, and thus the counting statistics will give large uncertainties. As shown in Figure 59, it appears that the $740 \mathrm{keV}$ and $778 \mathrm{keV}$ peaks gave accurate results in Singles mode while the $181 \mathrm{keV}$ and $141 \mathrm{keV}$ peaks gave mixed results. 


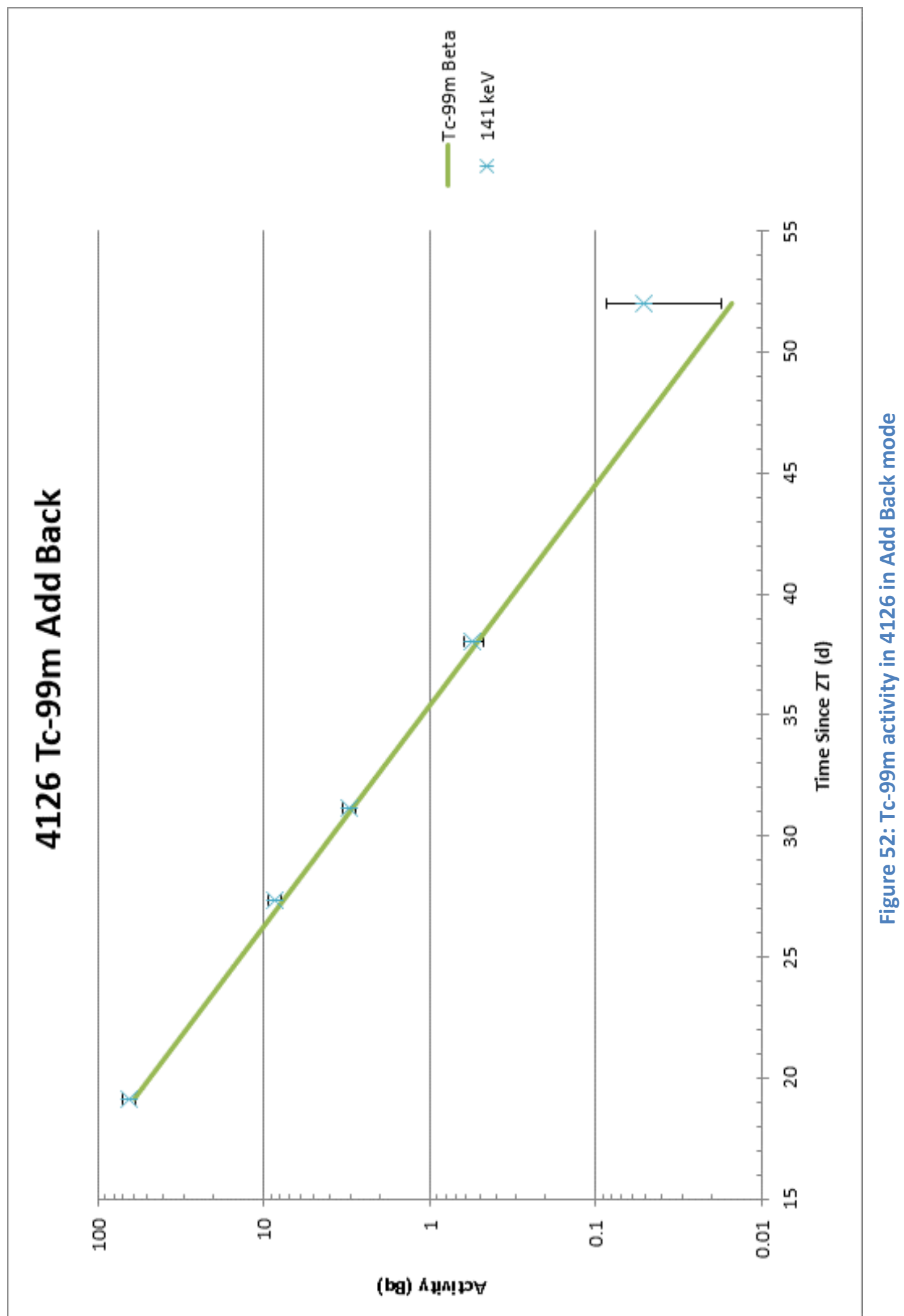




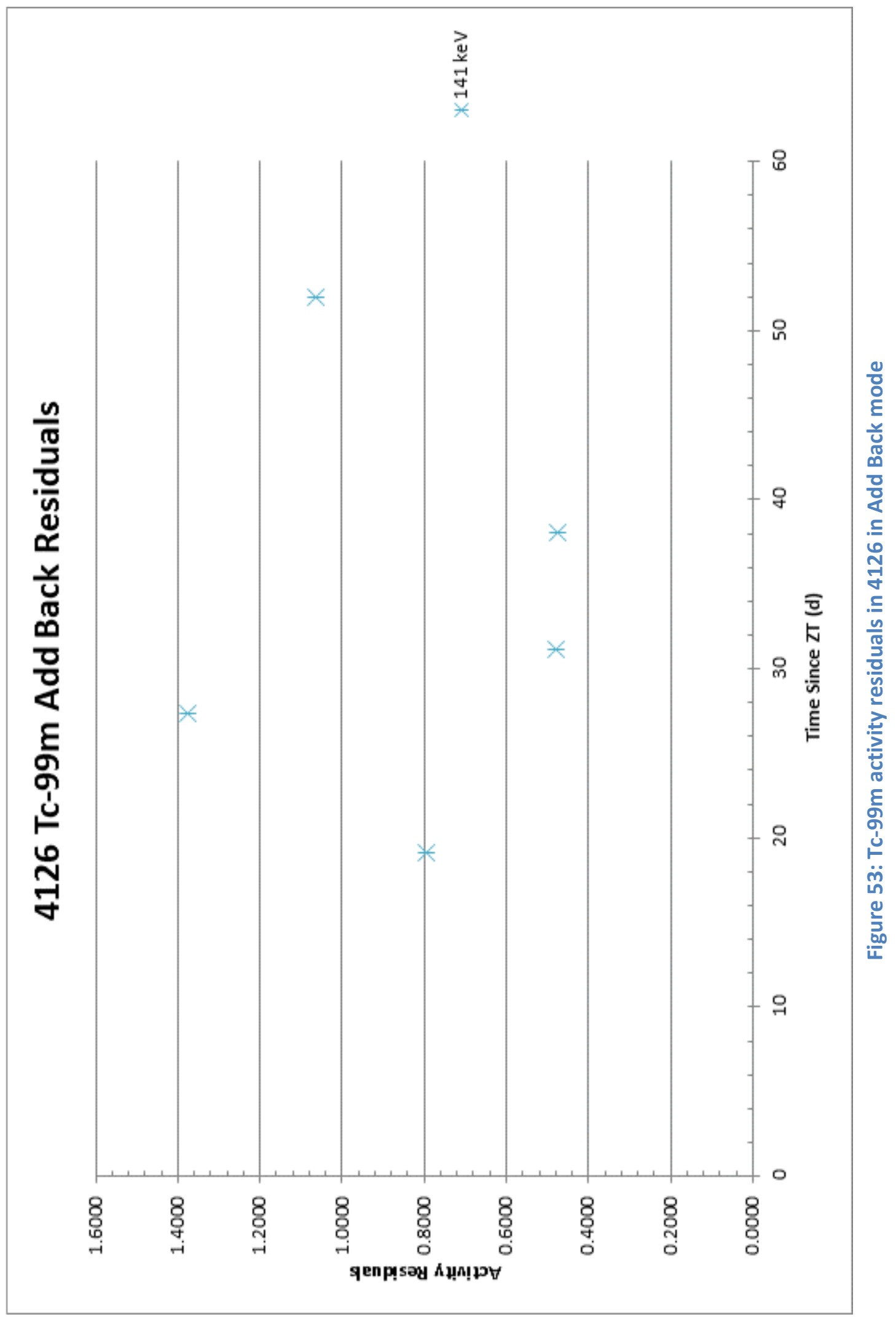




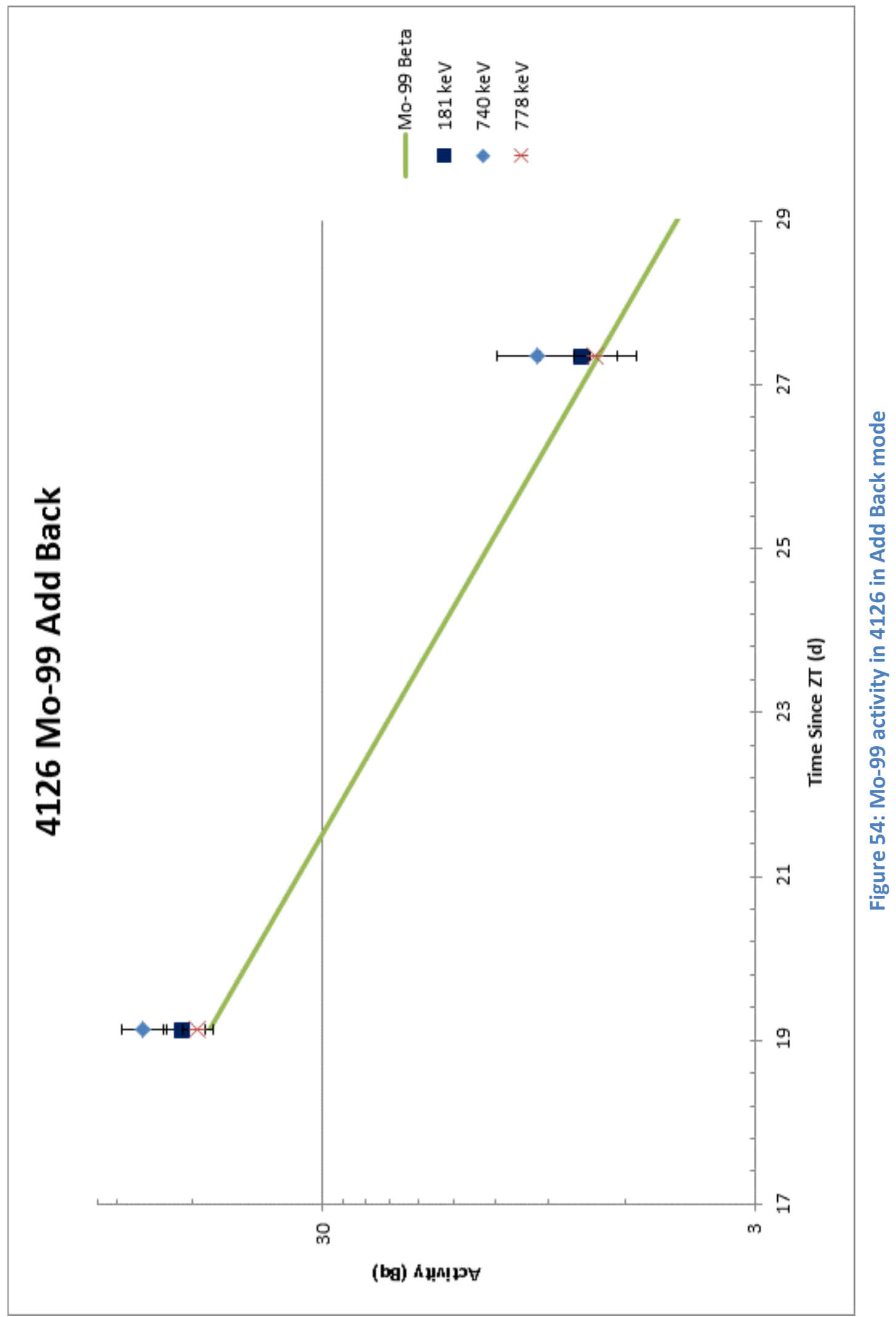




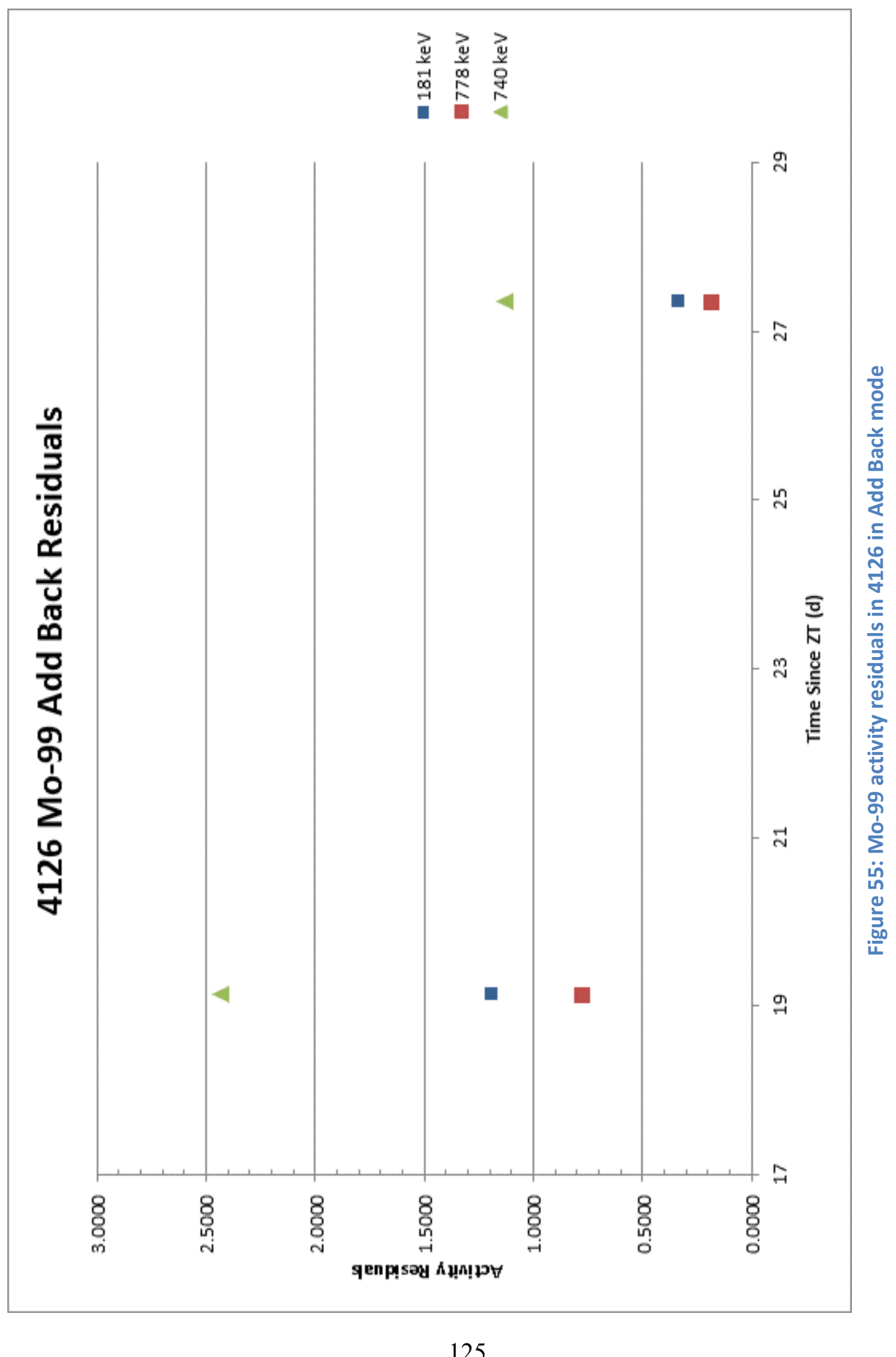




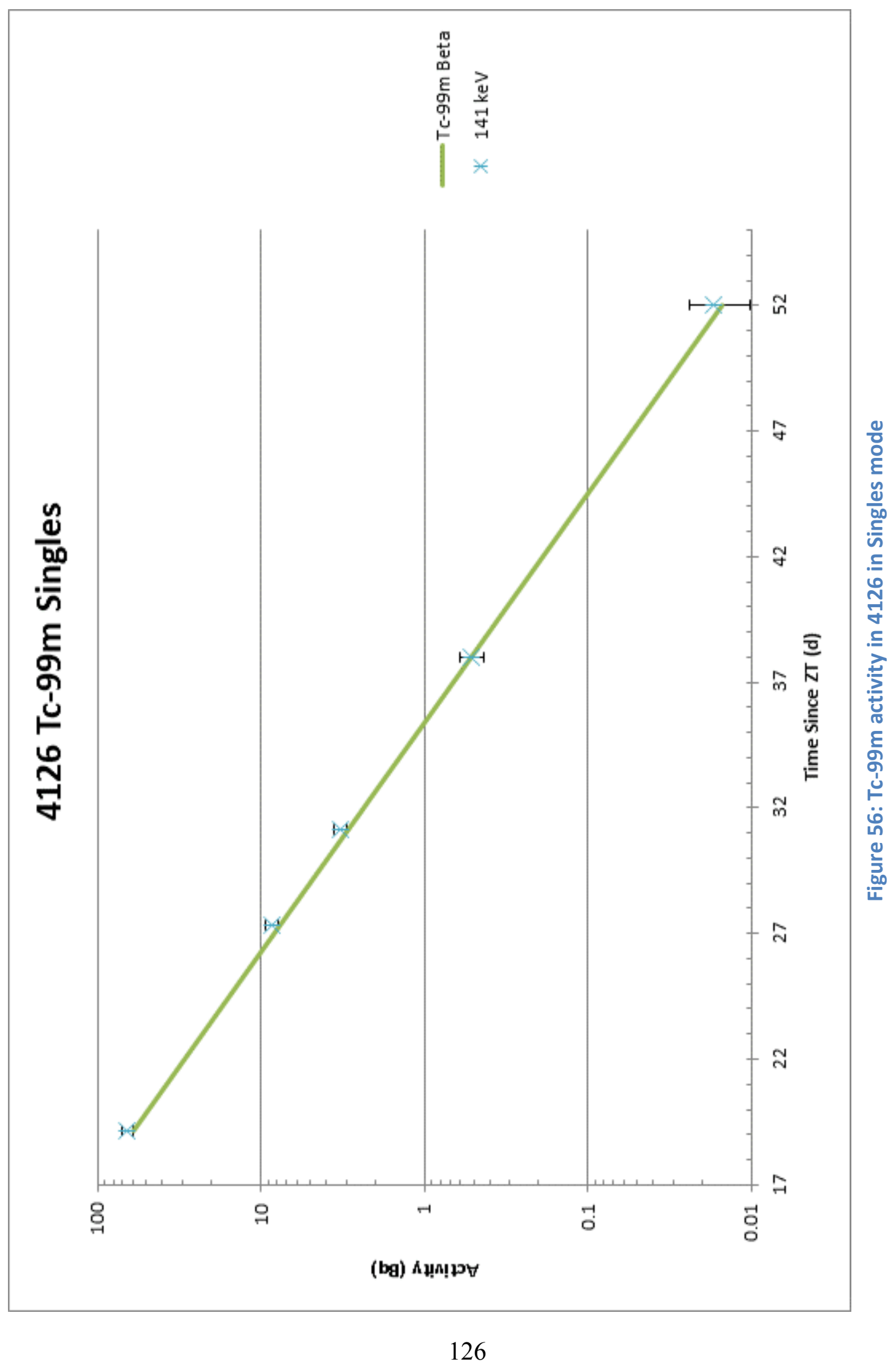




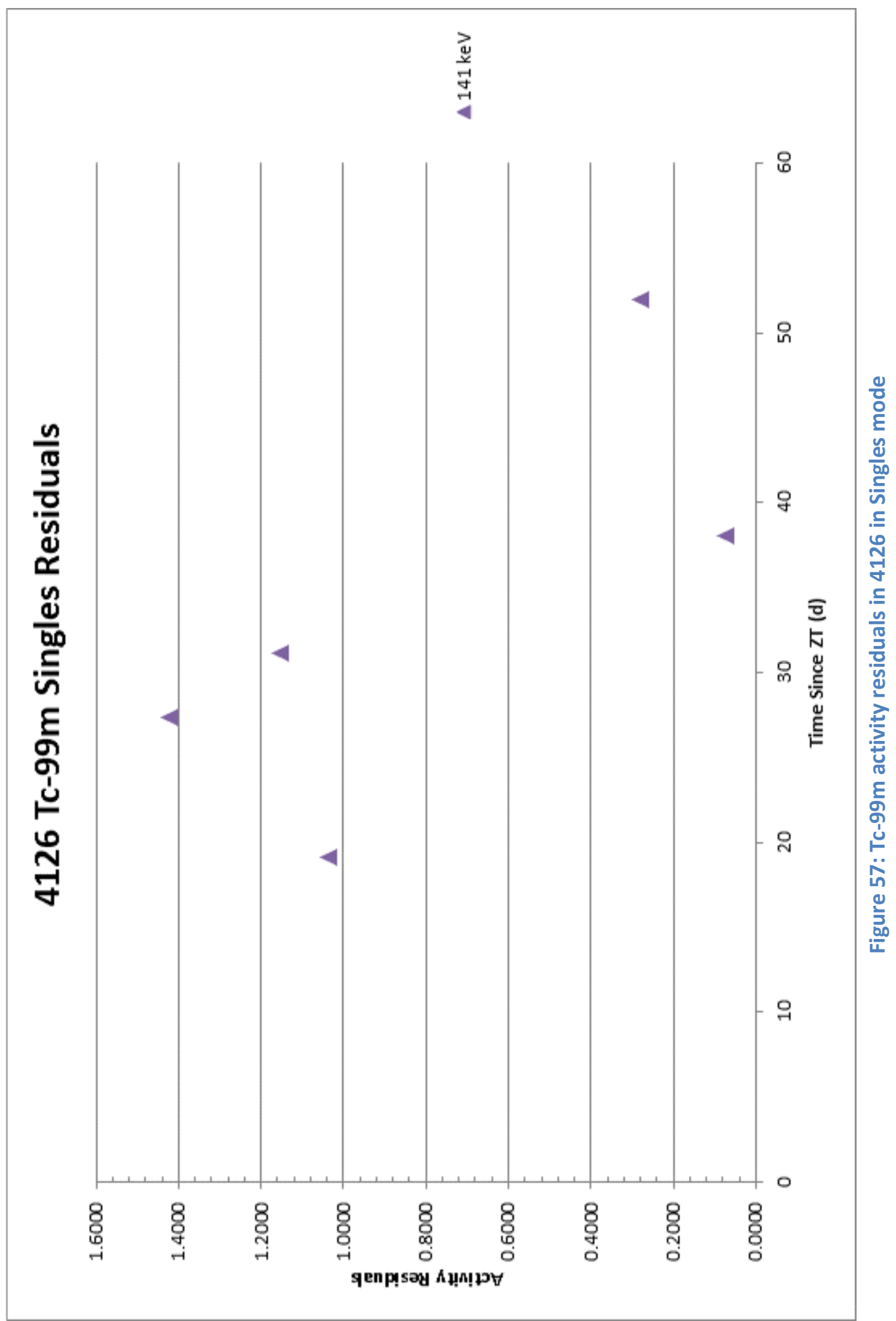




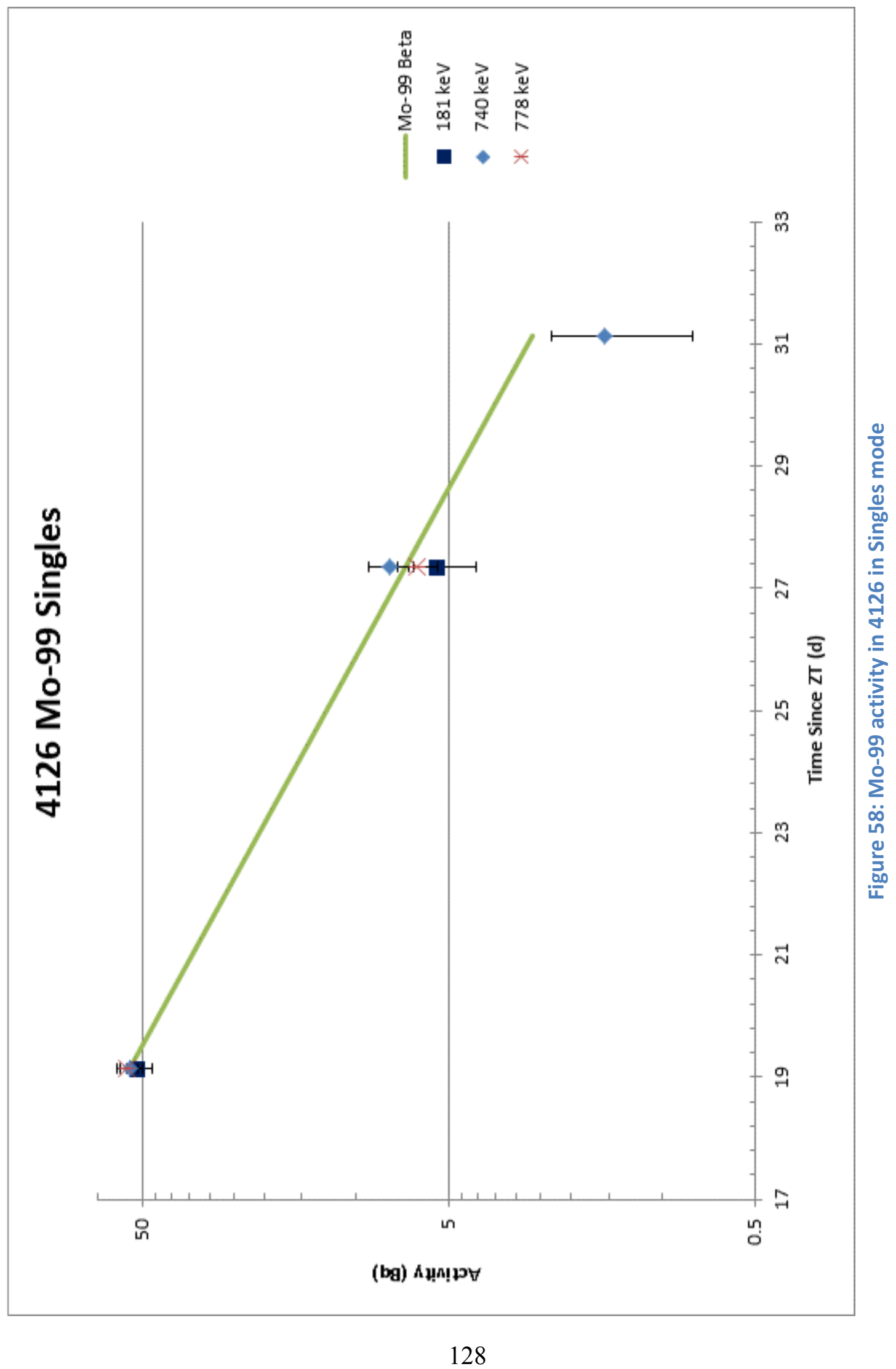




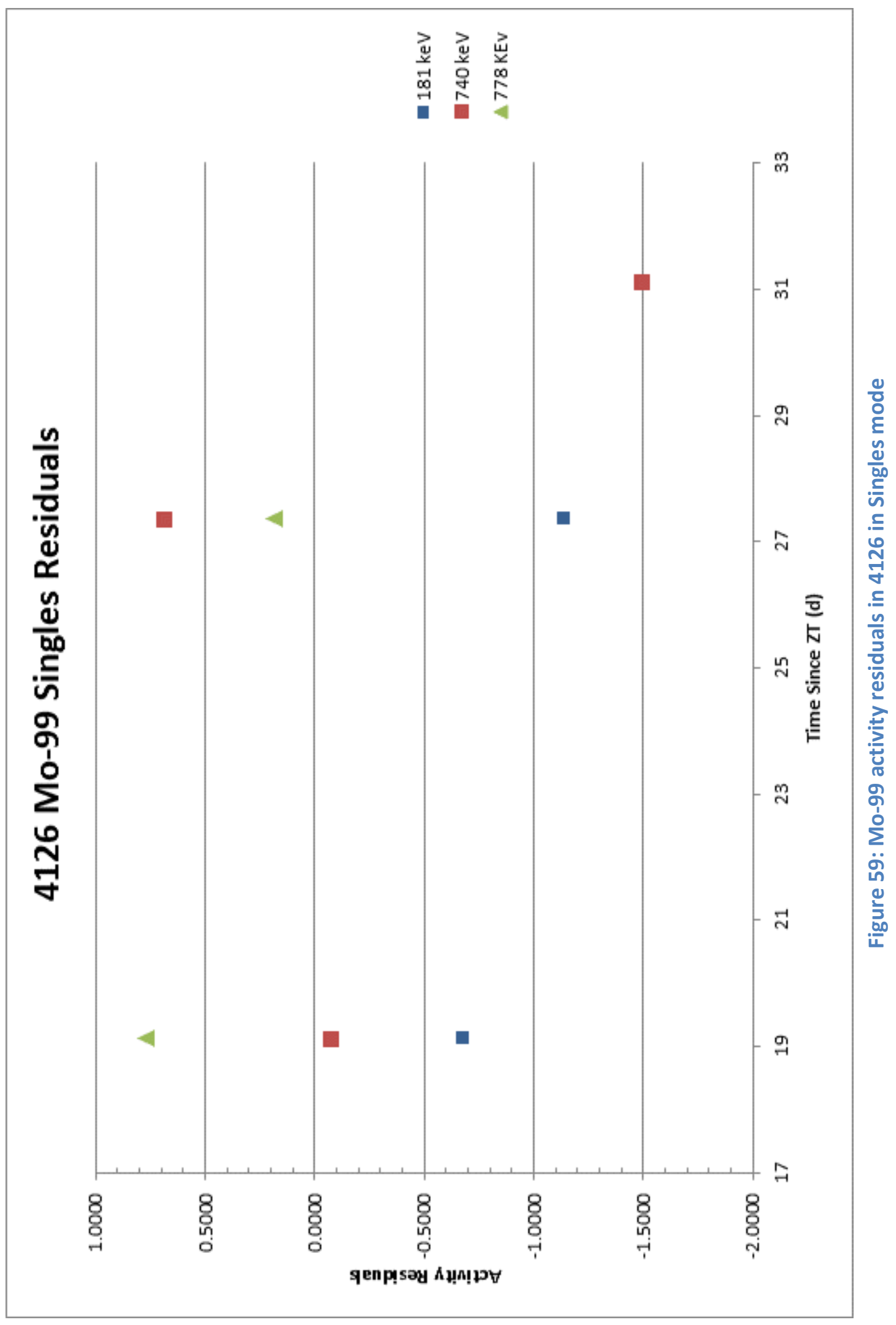


The gamma-gamma coincidence plots are arranged such that the first photon is gated on the detector displayed in the graph's title. As an example, looking at Figure 61, the red square is taken by gating Kerri on the $1596 \mathrm{keV}$ photon and observing the $329 \mathrm{keV}$ peak in Misty. Using this mode of counting, it is apparent that none of the coincidences give consistently accurate results for determining the activity of ${ }^{140}$ La. As shown in Figure 61 and Figure 63, all of the observed coincidences produced results that were too high when compared with beta counting except for the coincidence between the $1596 \mathrm{keV}$ photon and the $816 \mathrm{keV}$ photon, which produced results that were low when compared with beta counting. 


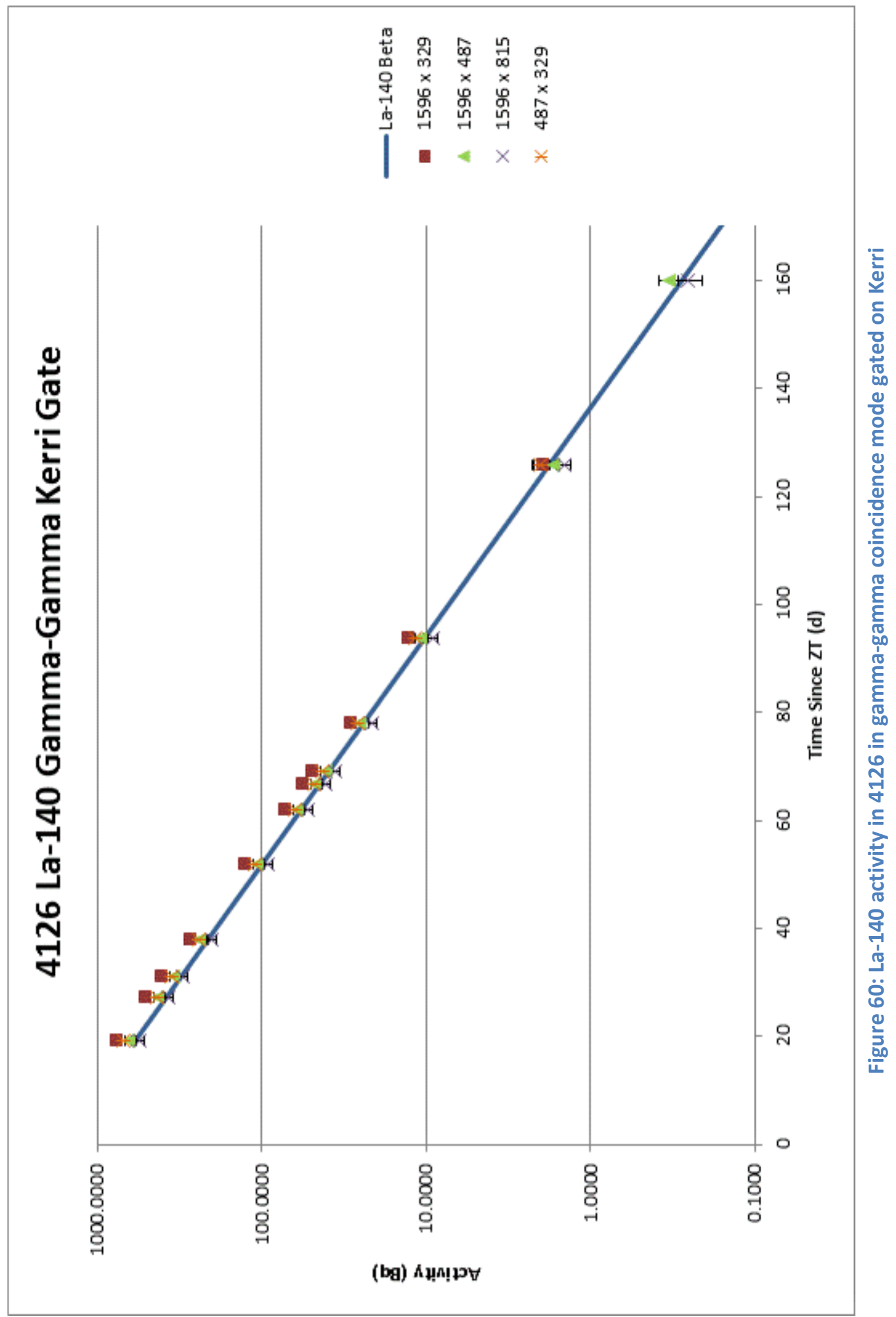




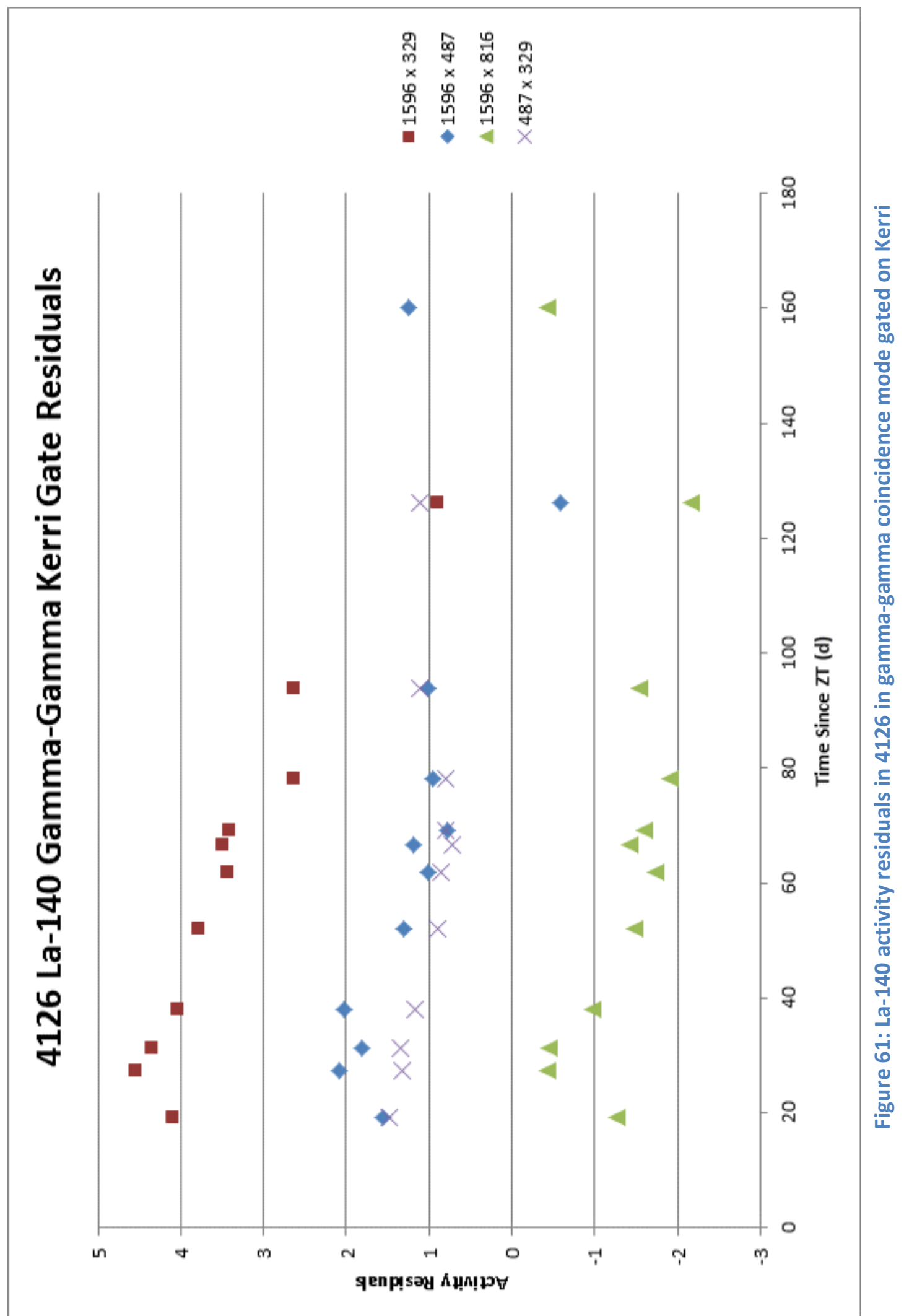




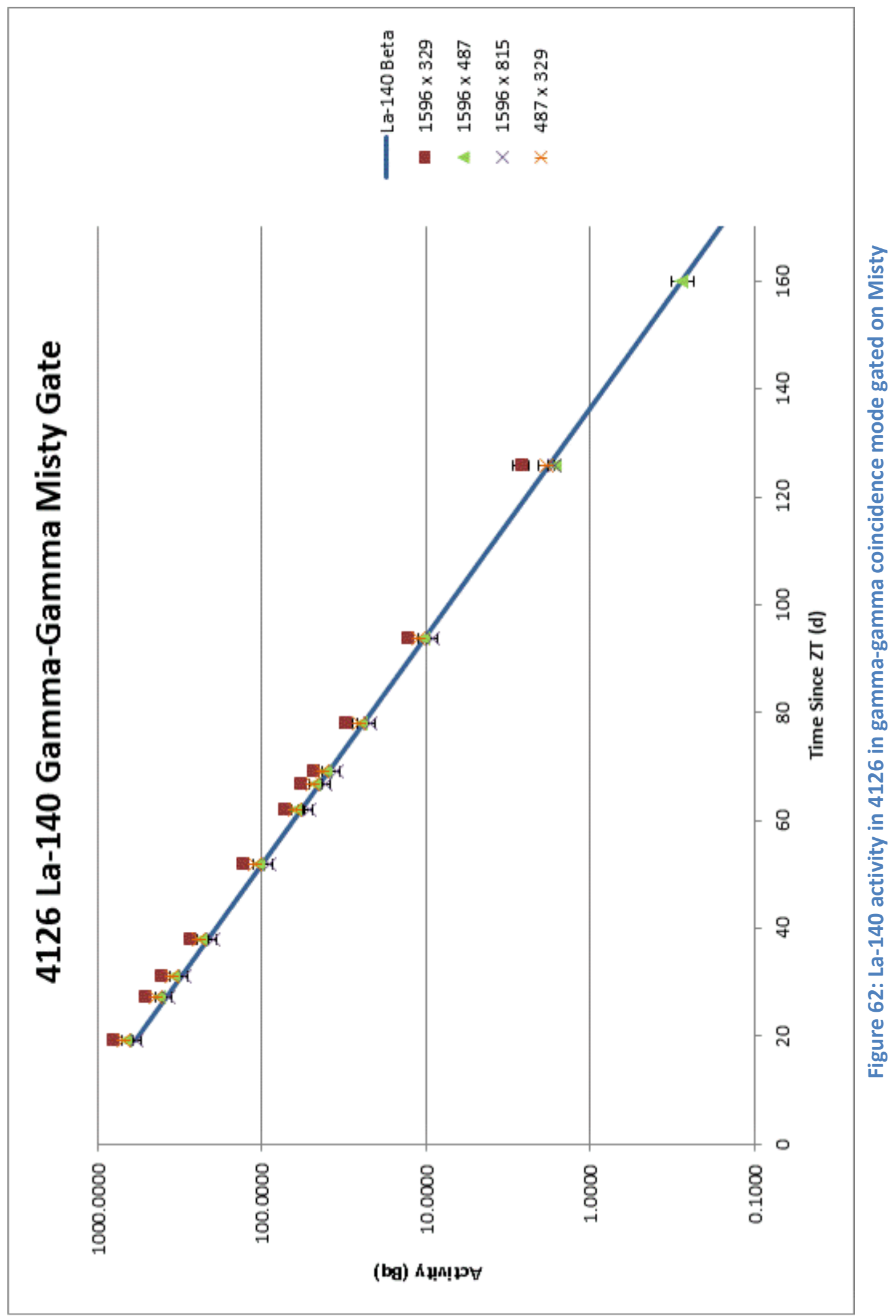




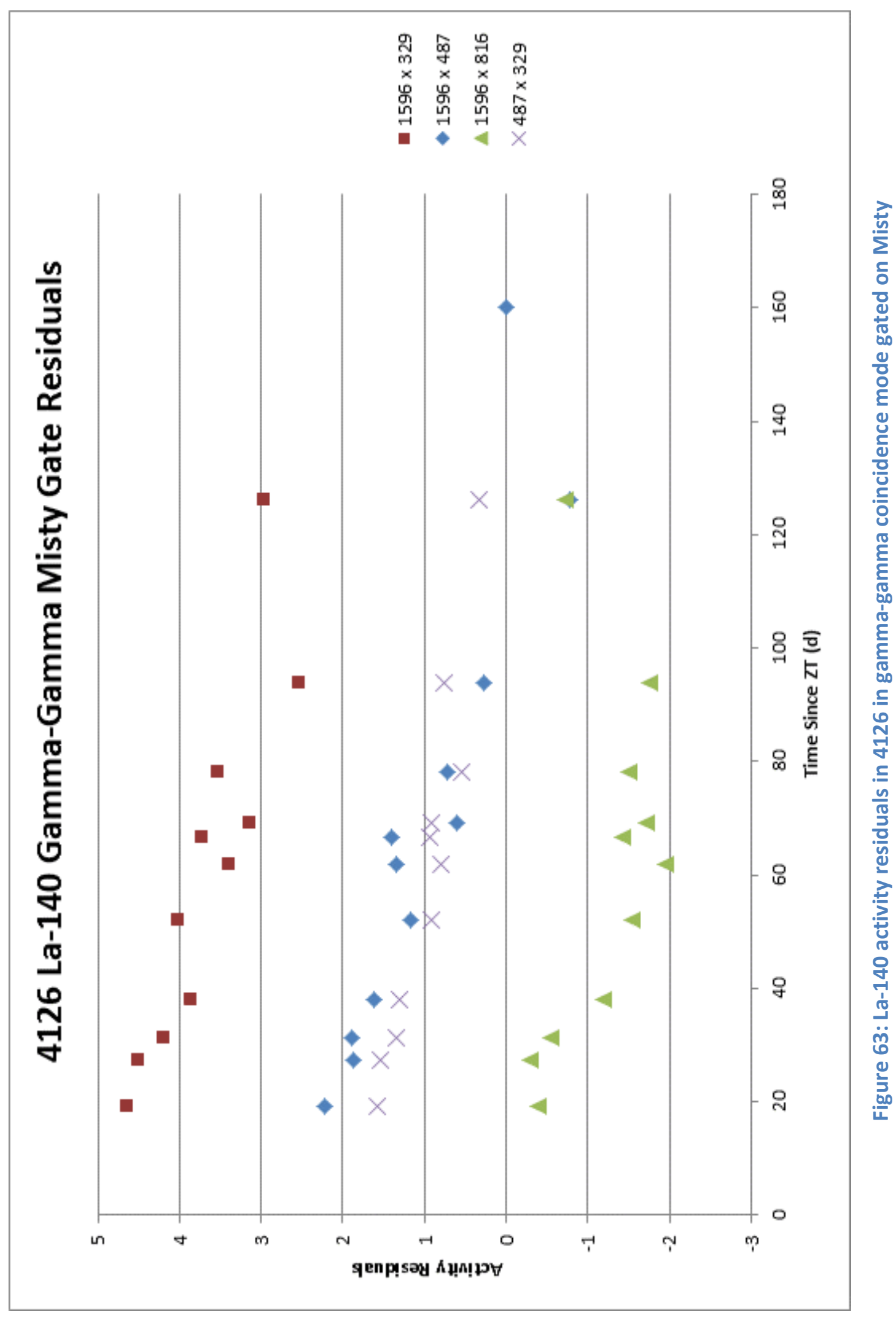


As shown in Figure 65 and Figure 67, the Gamma-Gamma coincidence data for ${ }^{140}$ Ba give accurate results for both gating schemes. All data points in the residuals plots fall within $-1 \sigma$ to $1 \sigma$. There may be a slight downward trend in the residuals plots. 


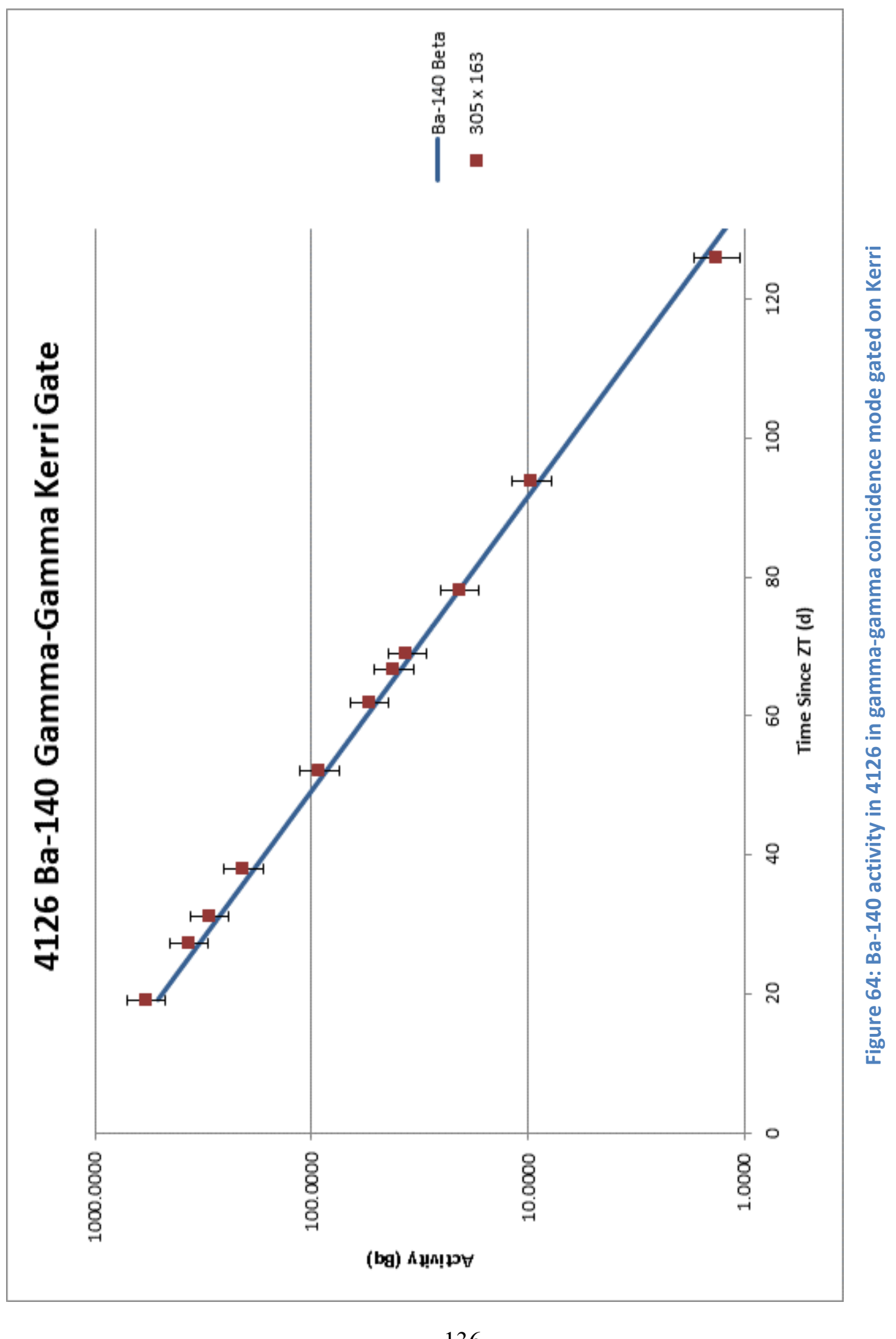




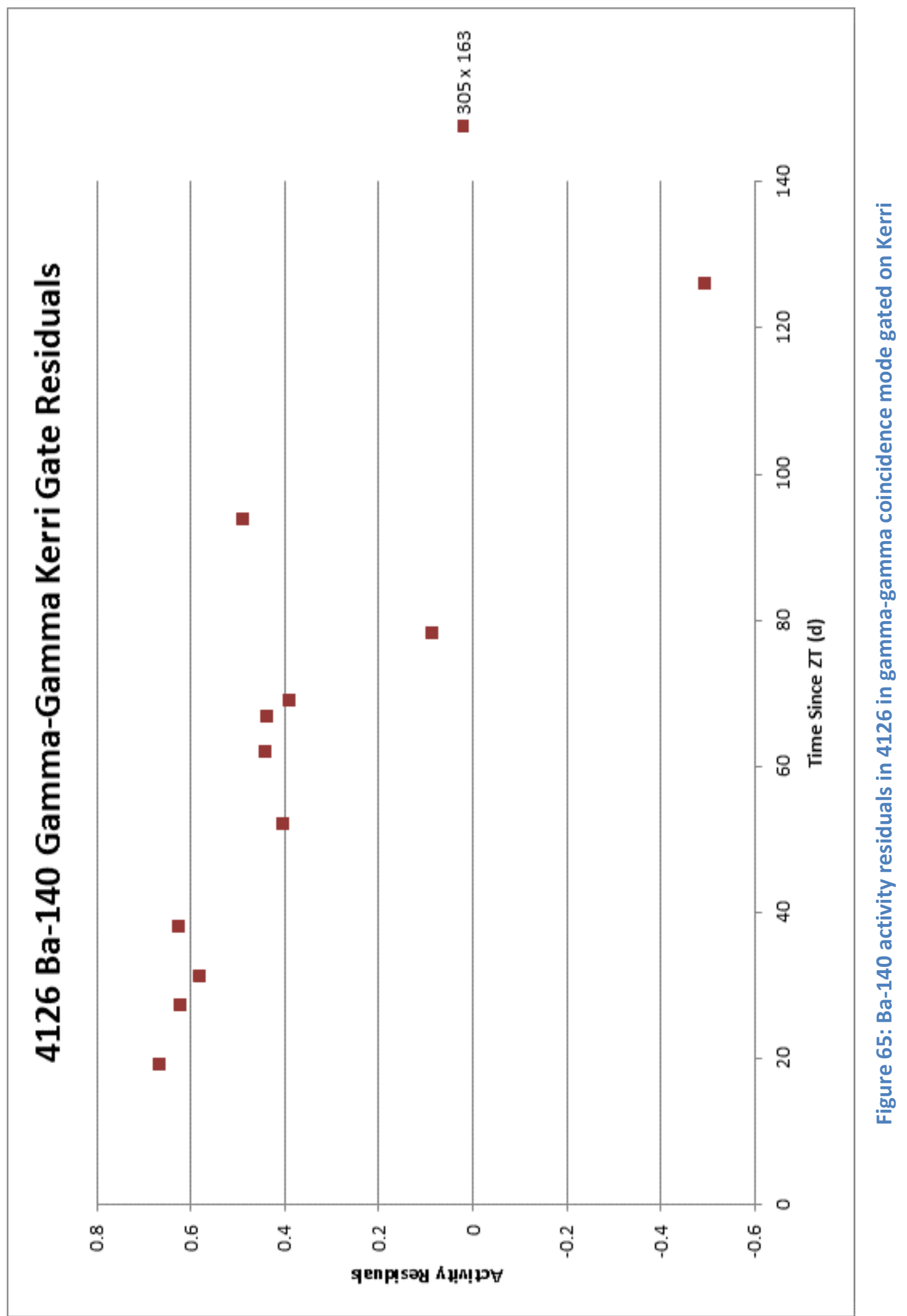




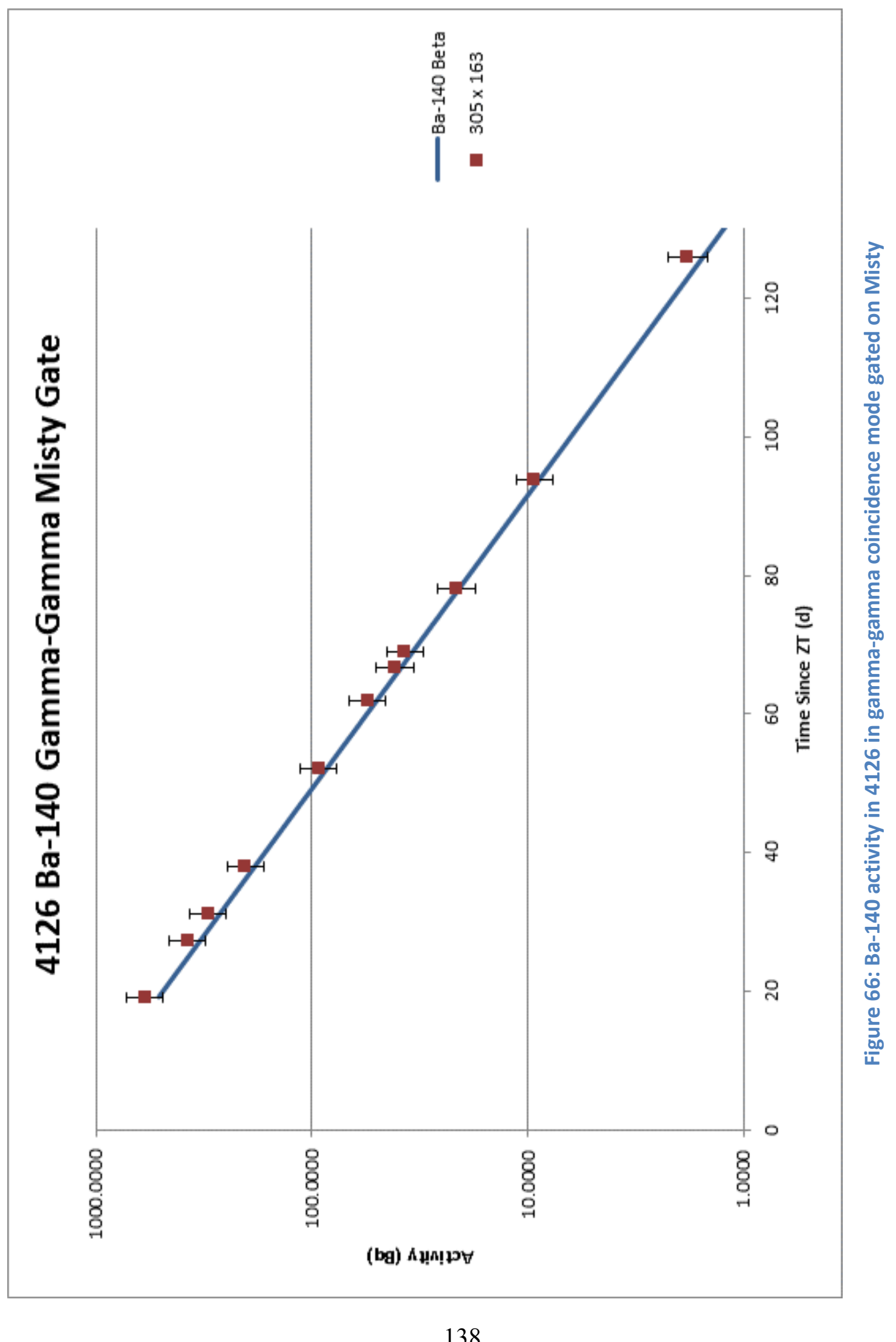




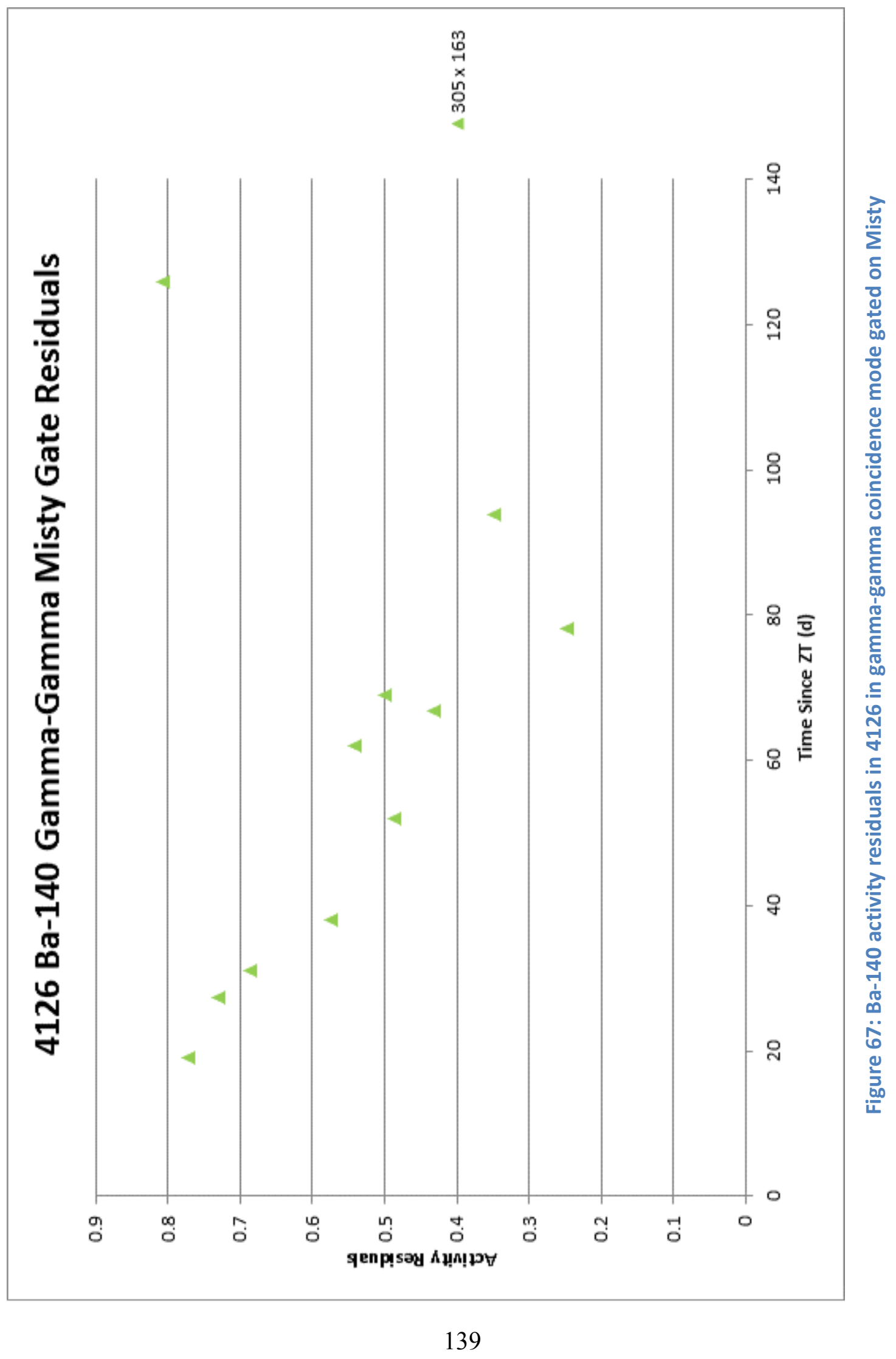


The gamma-gamma coincidence data for ${ }^{99}$ Mo show mixed results, but have a very limited number of data points. As seen in Figure 69 and Figure 71, the coincidence between the $740 \mathrm{keV}$ photon and the $141 \mathrm{keV}$ photon may give accurate results in both gating schemes. The coincidence between the $740 \mathrm{keV}$ photon and the $181 \mathrm{keV}$ photon show mixed results, and may not be accurate due to low peak areas in the fitting routines. 


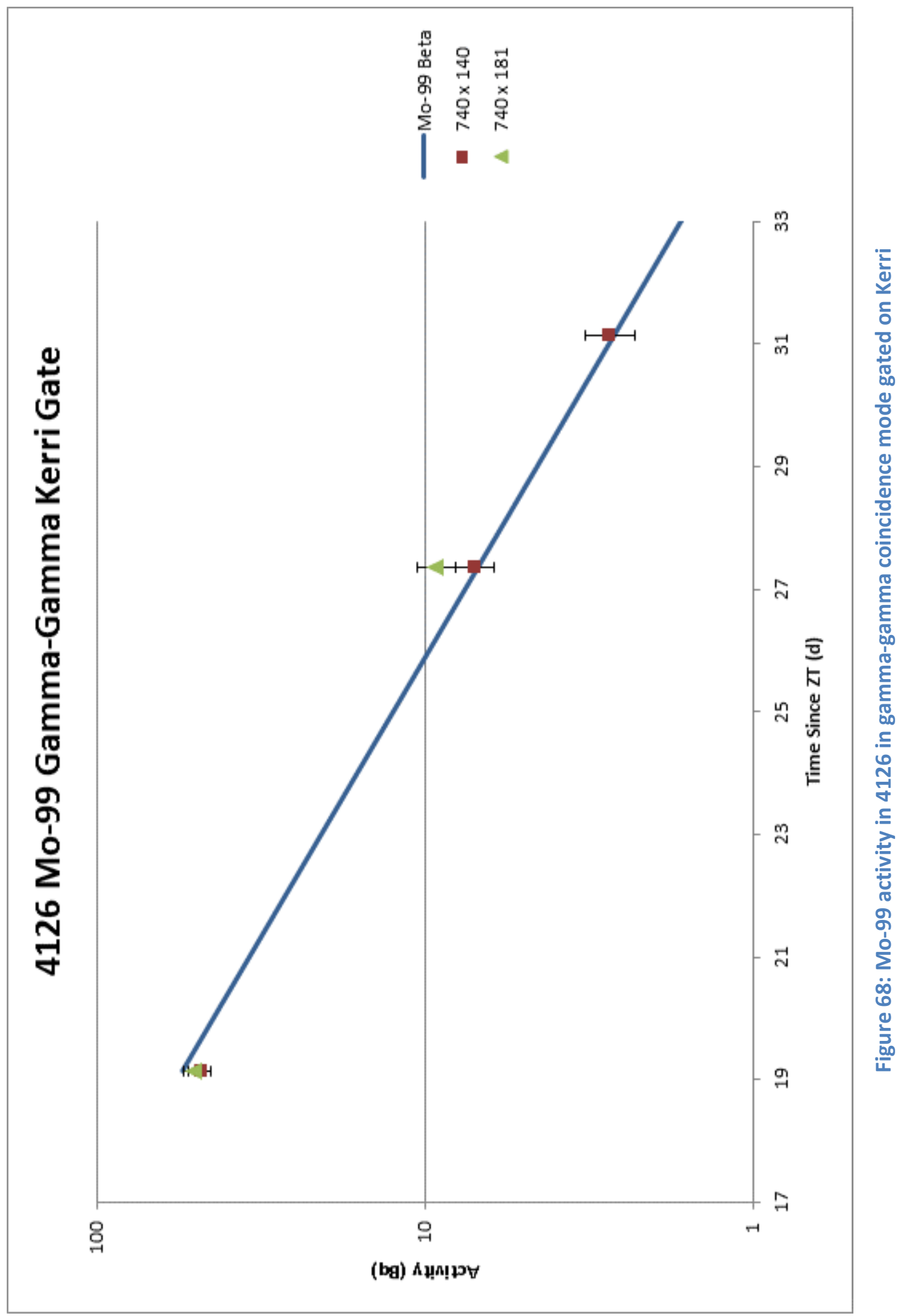




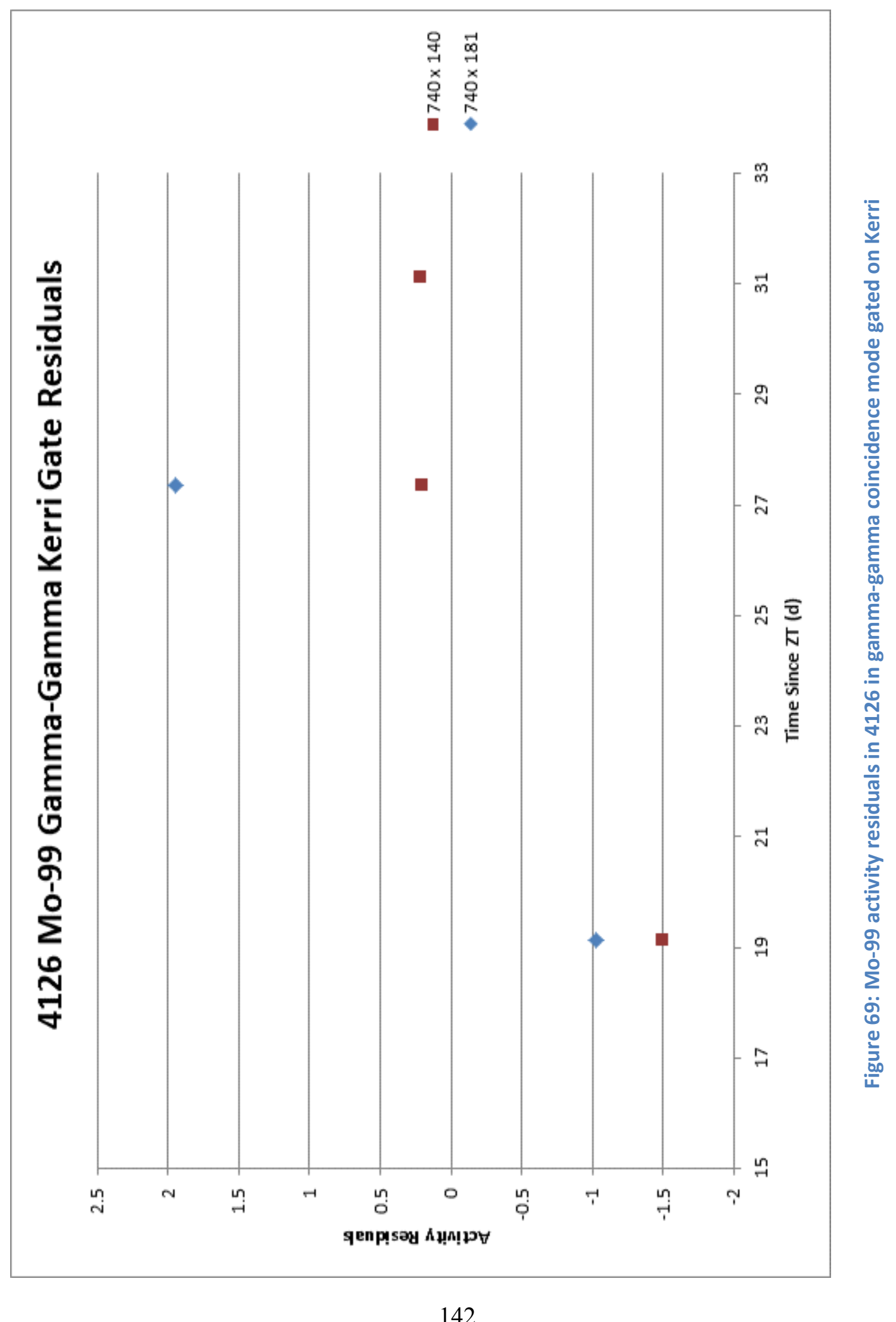




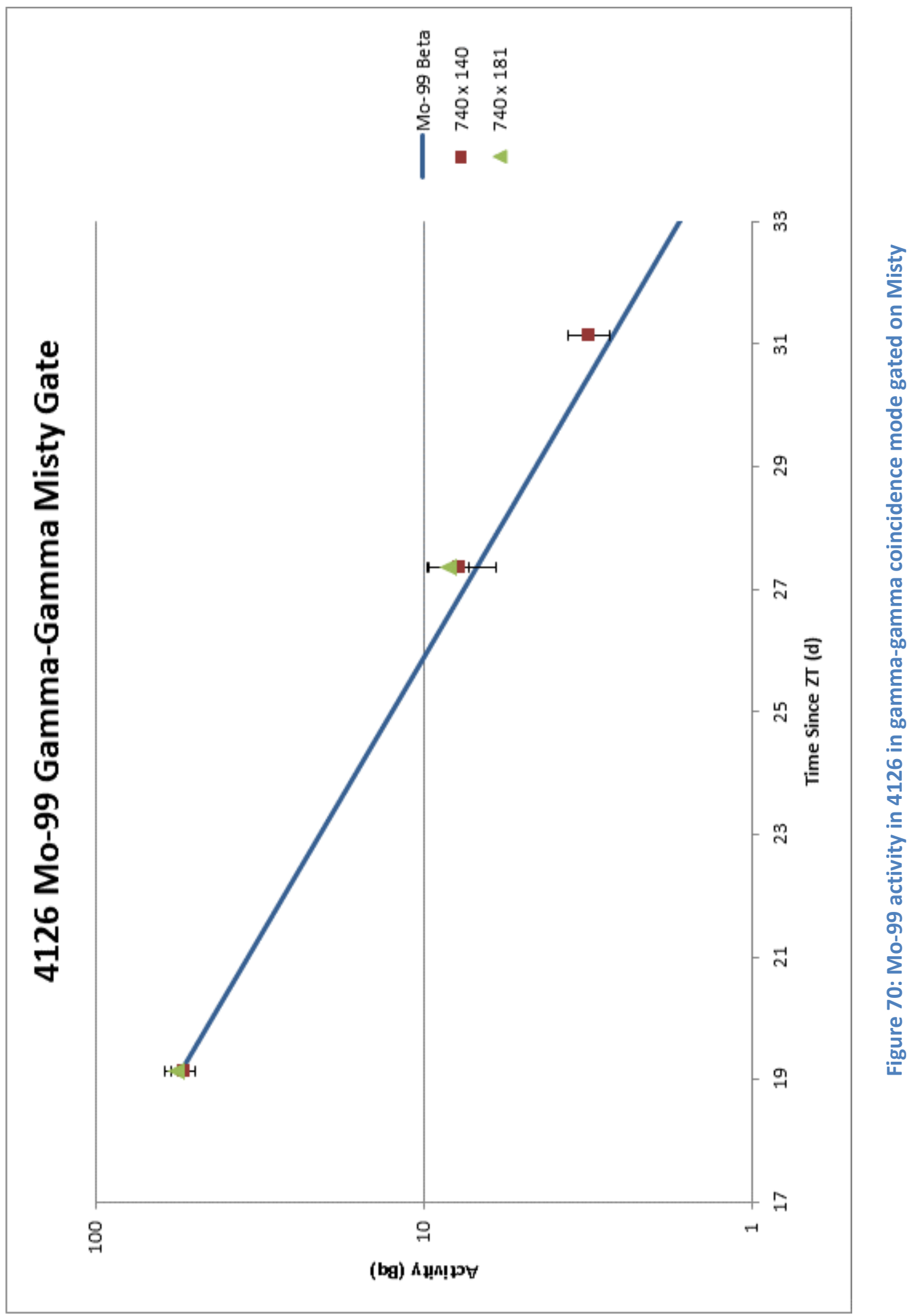




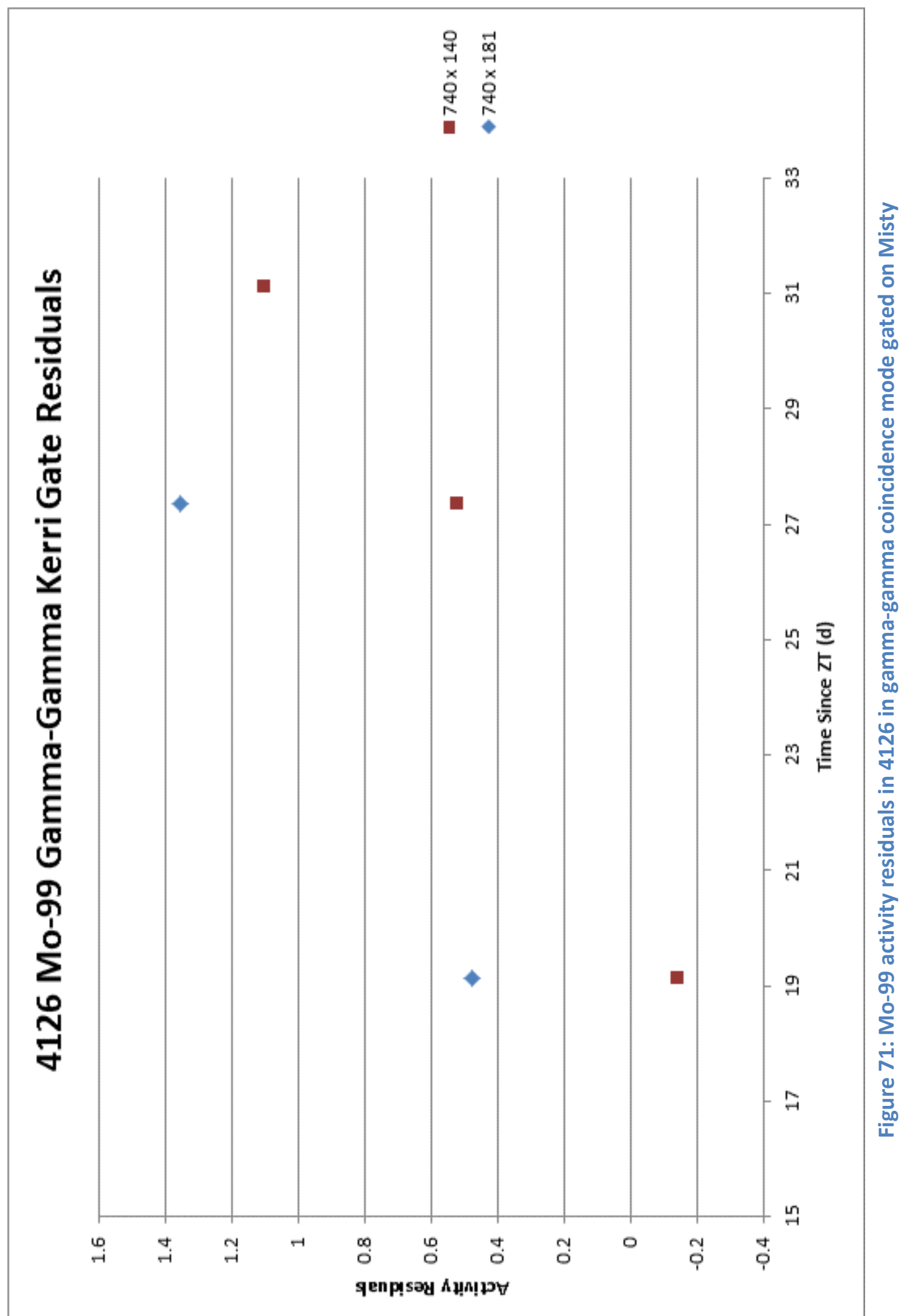




\subsection{Activity Calculation Results from Sample 4130}

As seen in Figure 72 through Figure 79, both Add Back and Singles modes show good

agreement with the ${ }^{95} \mathrm{Zr}$ and ${ }^{95} \mathrm{Nb}$ activity estimations from beta counting. All the activity residuals fall within $-1 \sigma$ to $1 \sigma$. 


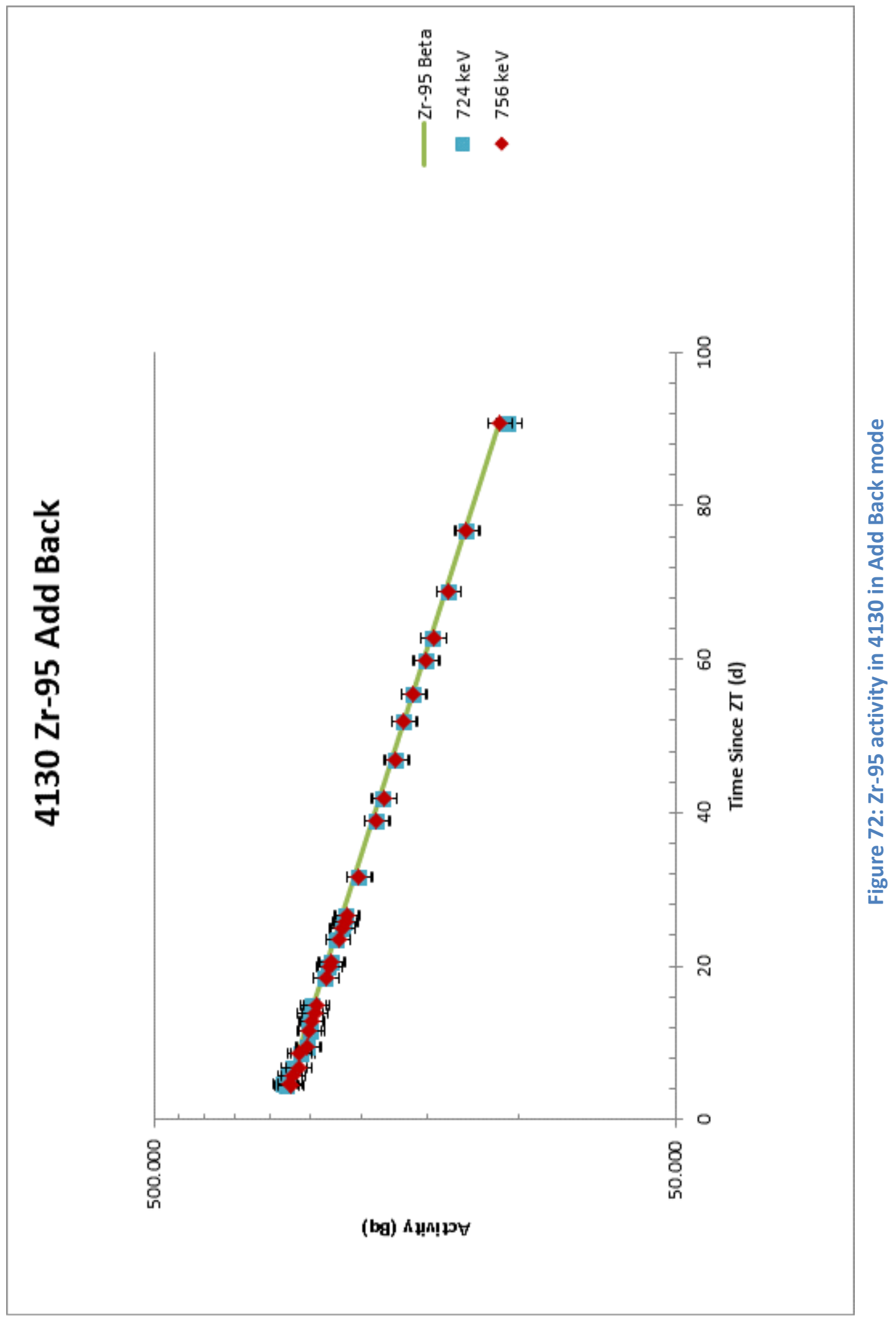




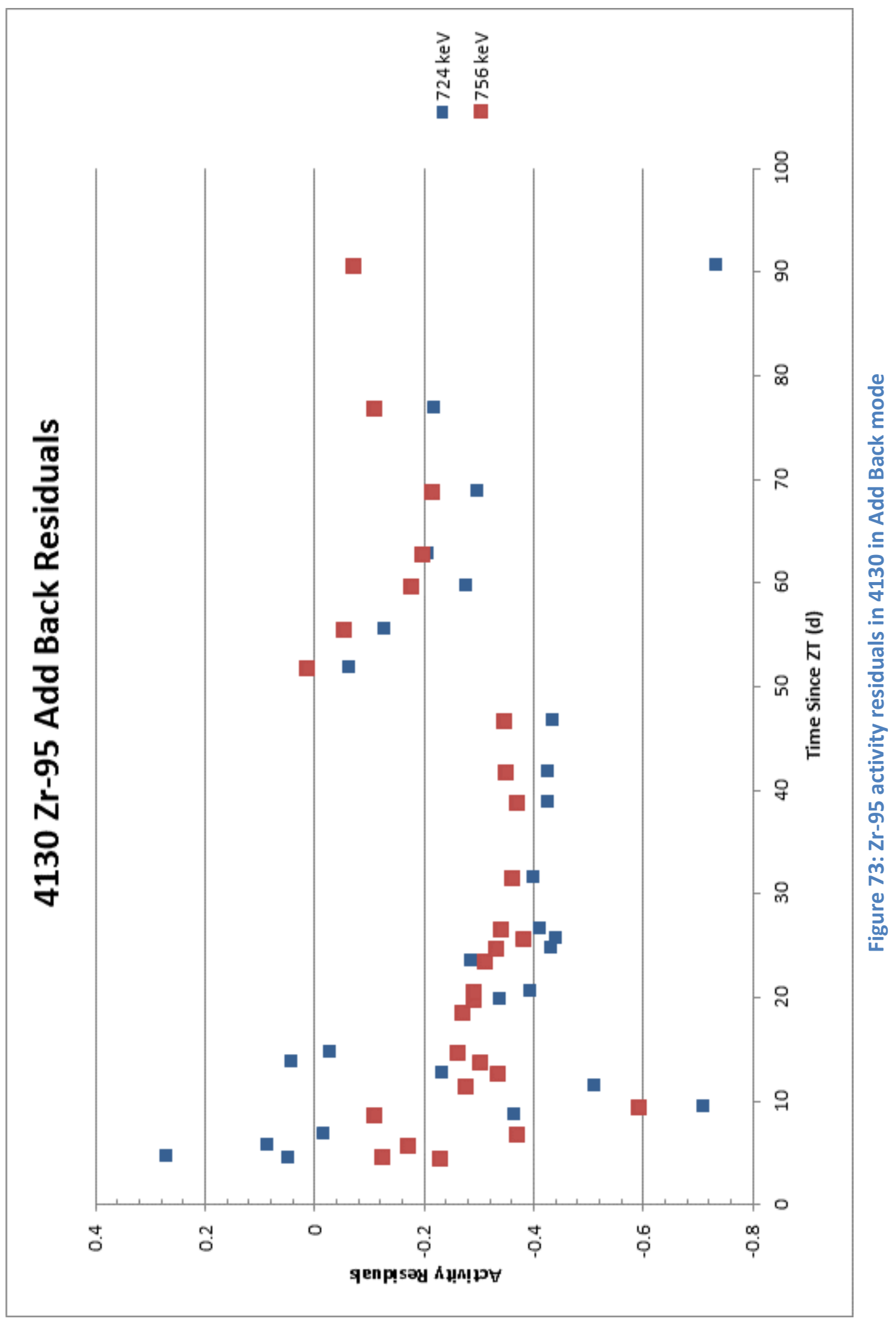




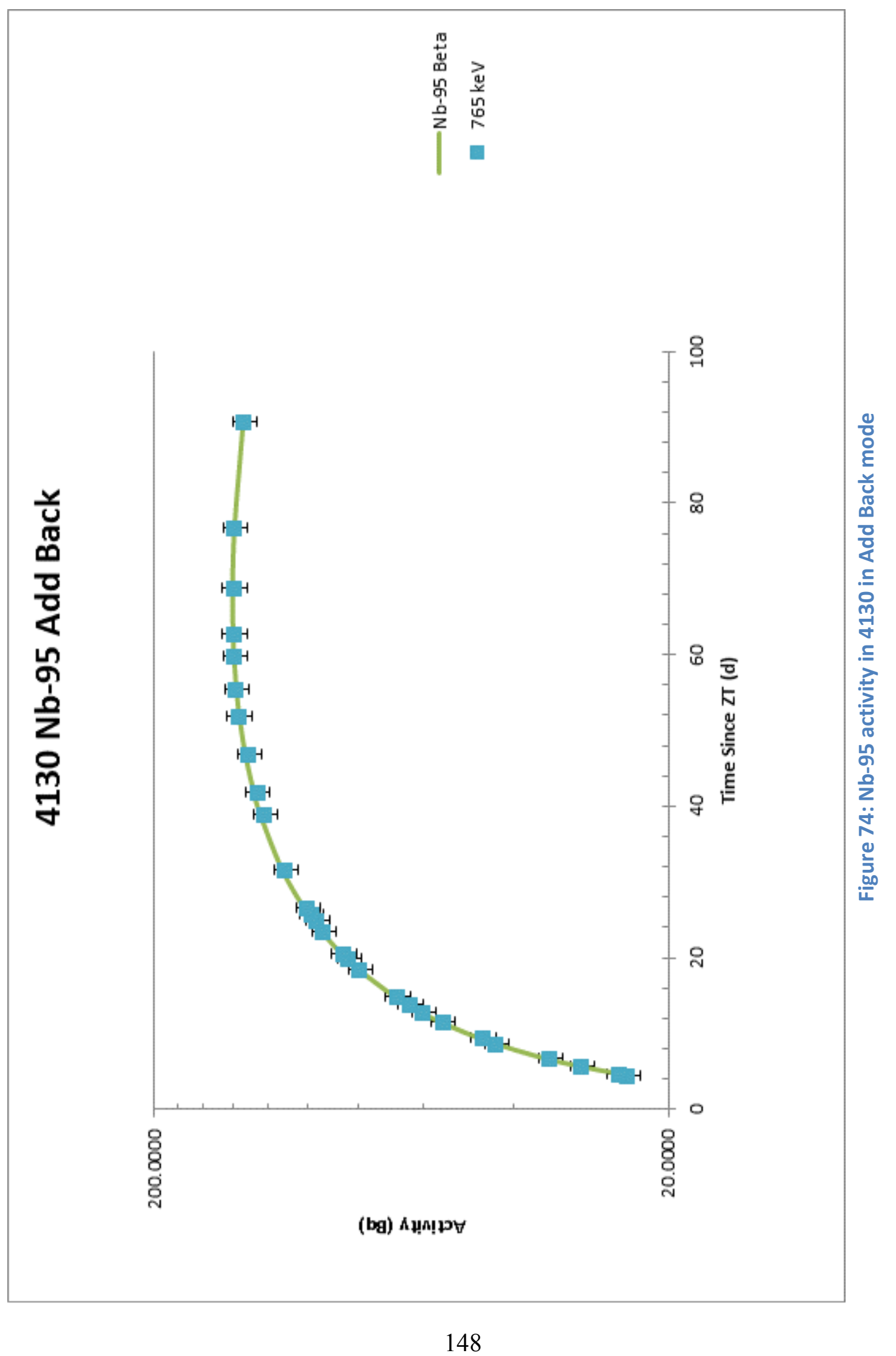




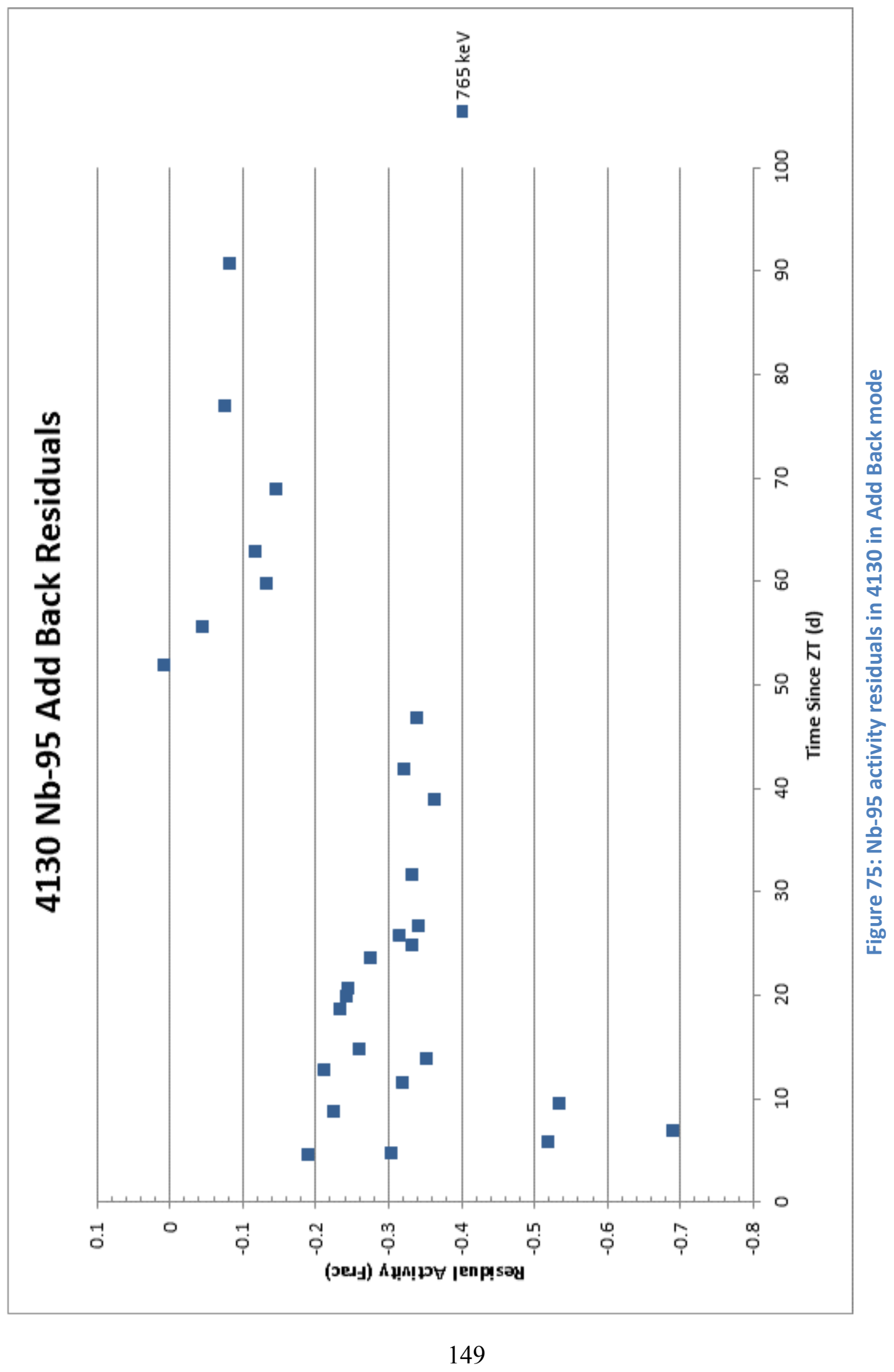




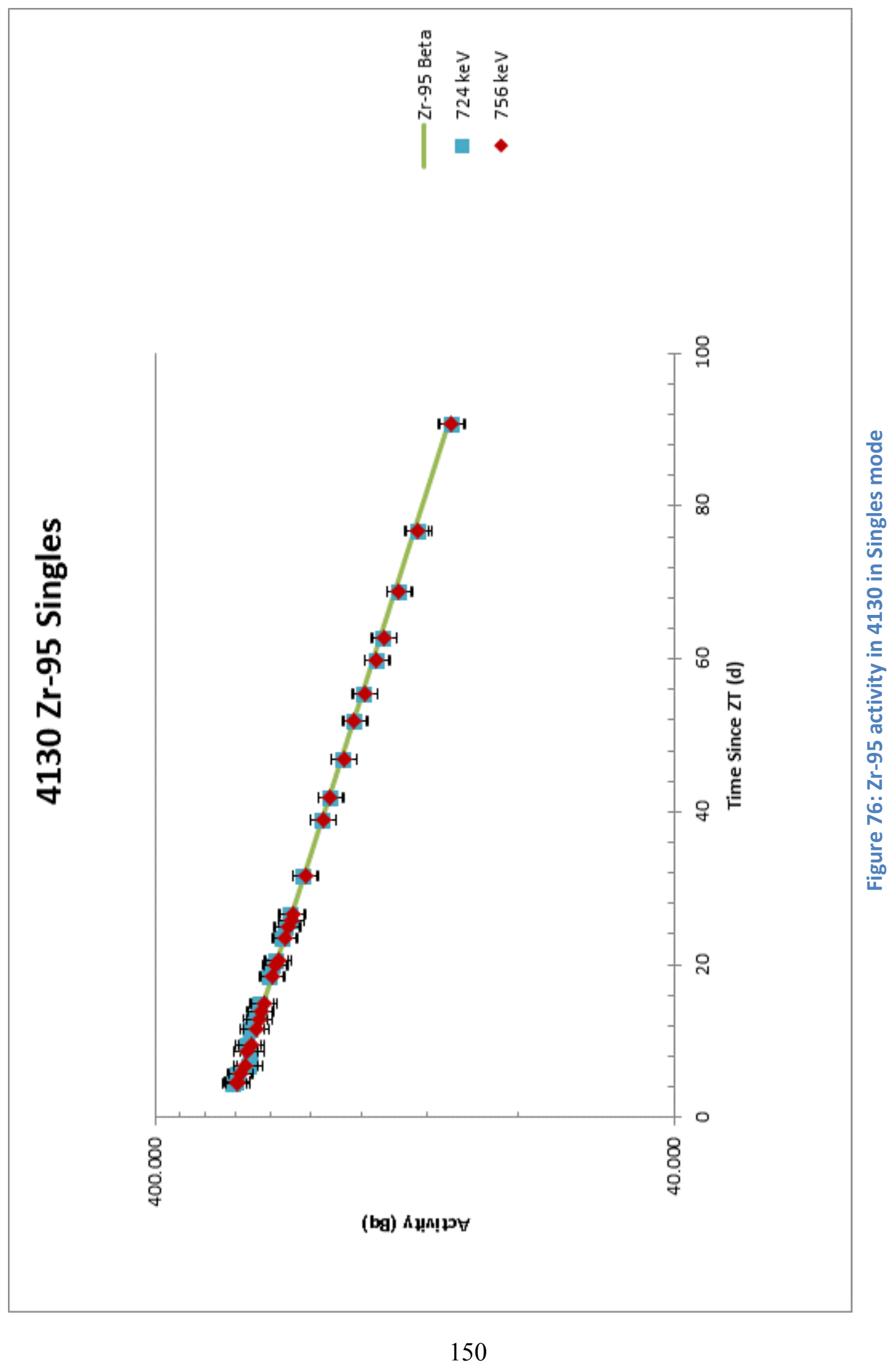




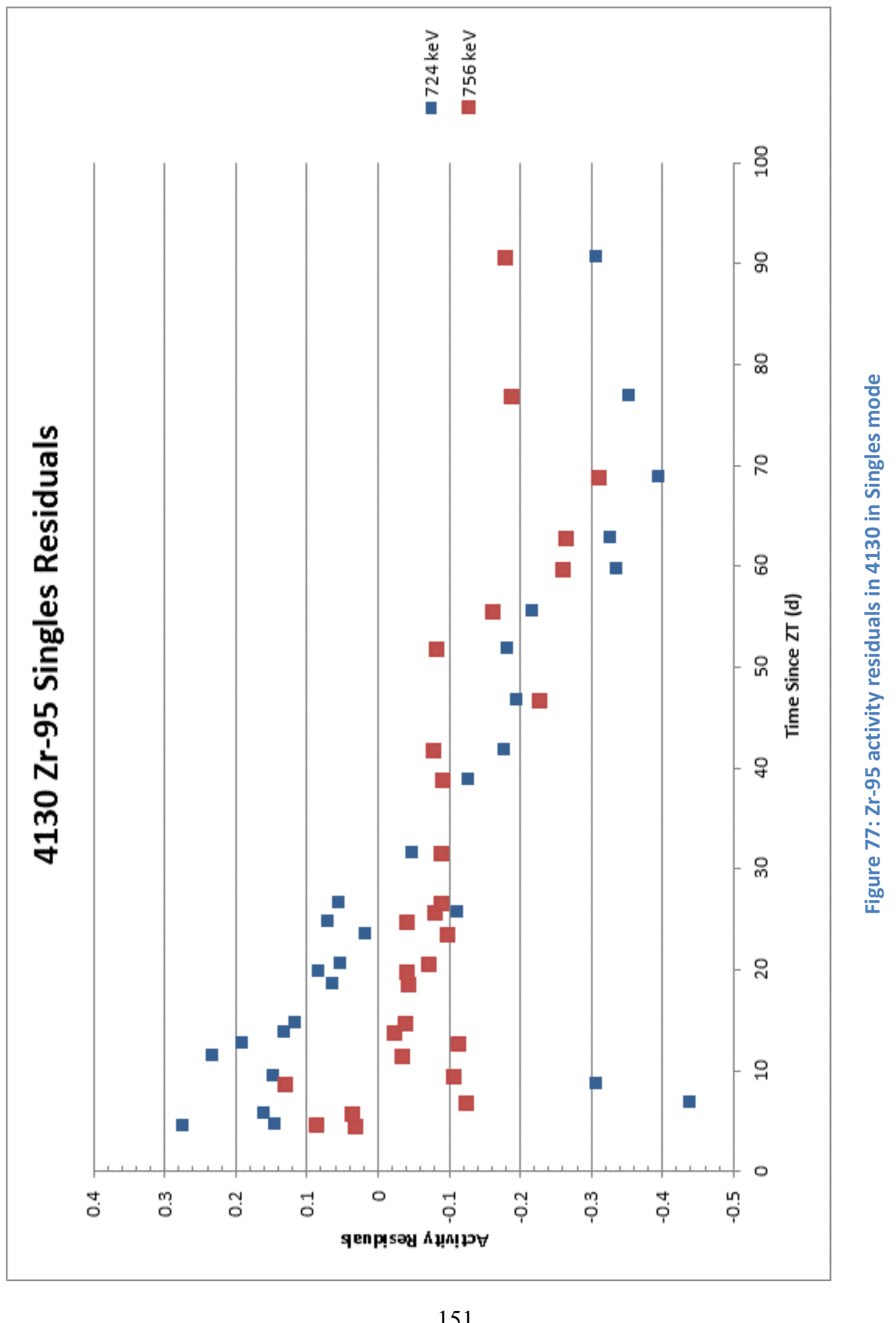




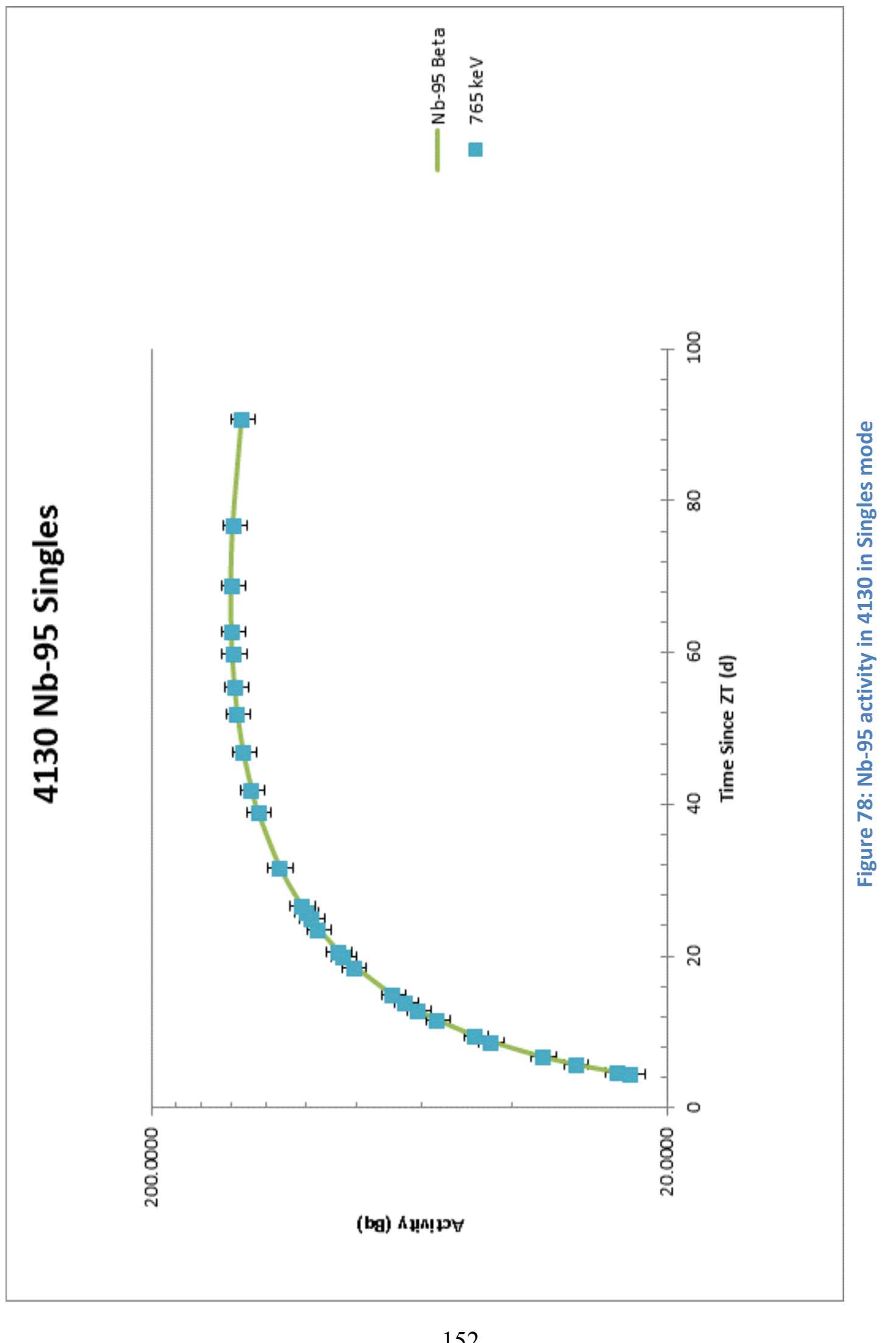




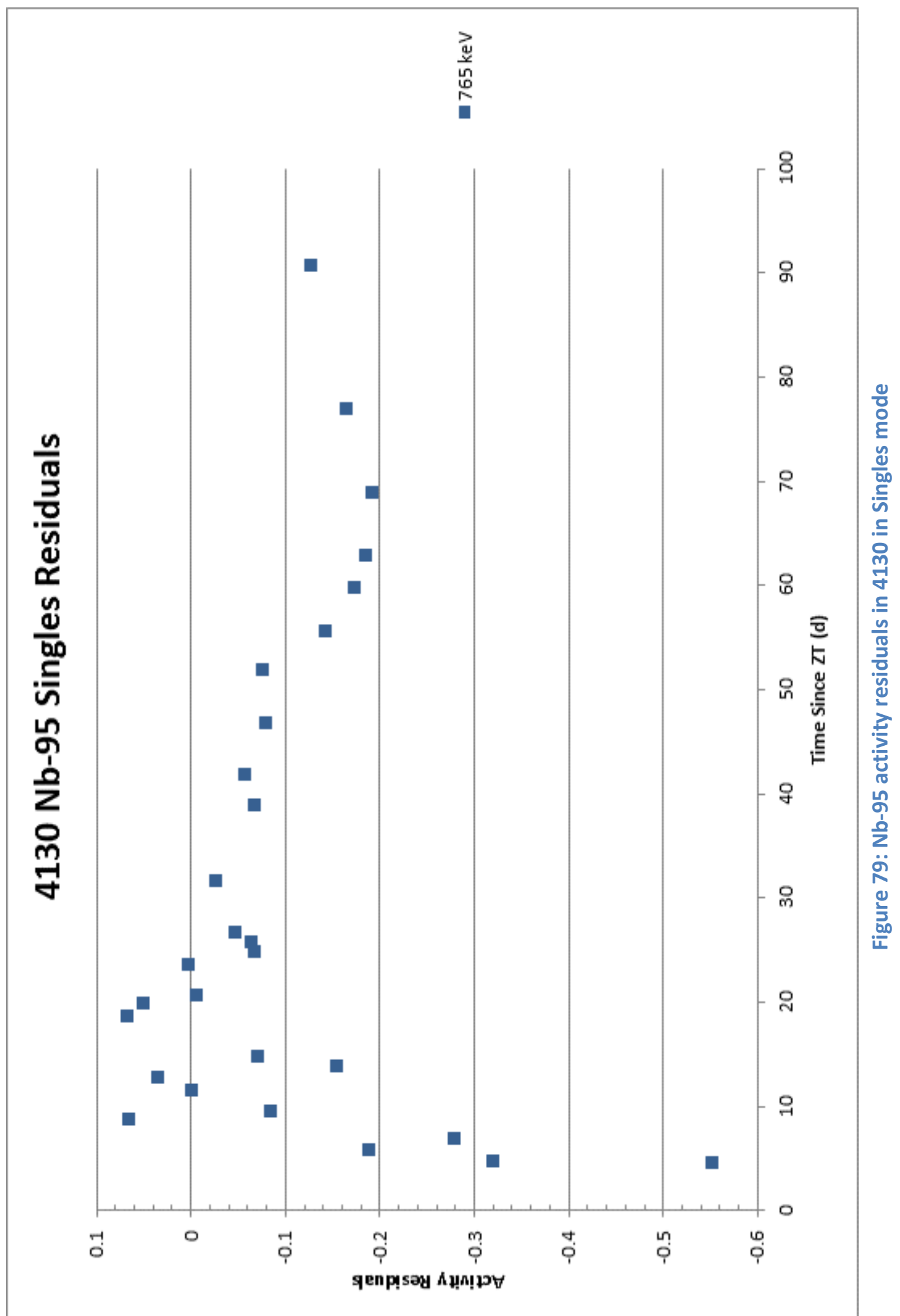


As shown in Figure 81, most of the ${ }^{140} \mathrm{Ba}$ Add Back data points agree with the beta counting activity estimations. The exception was the $305 \mathrm{keV}$ photon, which produced activities that were high when compared with the beta counting estimate. As with the 4126 sample, the $163 \mathrm{keV}$ photon has been corrected for the ${ }^{235} \mathrm{U}$ emission. This was done by looking at the last spectrum in the 4126 sample and observing the peak ratio between the $186 \mathrm{keV}$ peak and the $163 \mathrm{keV}$ peak. This spectrum was chosen because it was the oldest spectrum and it was assumed that the observable portion of the $163 \mathrm{keV}$ emission from ${ }^{140} \mathrm{Ba}$ was absent. The 186 $\mathrm{keV}$ peak area was then observed in the last spectrum of the 4130 sample and the estimated count rate of the $163 \mathrm{keV}$ peak that originated from ${ }^{235} \mathrm{U}$ was calculated. This estimated count rate was then subtracted from all the 4130 spectra because the ${ }^{235} \mathrm{U}$ activity can be assumed to be constant for these counts. The Singles mode data for ${ }^{140} \mathrm{Ba}$ show consistent results with the estimations from beta counting from all photons, as demonstrated in Figure 85.

The ${ }^{140}$ La Add Back spectra are not consistent when compared with beta counting. As shown in Figure 83, all activity calculations are consistently above the estimated activity from beta counting. The $487 \mathrm{keV}, 816 \mathrm{keV}$, and $1596 \mathrm{keV}$ photon residuals spectra show a downward swing near the beginning of the counts and then level off. This is probably due to errors in the dead-time calculations. The dead-time for these photons was observed to be around $15 \%$ in the earliest spectrum in Add Back mode, which can produce skewed results given the approximations that were used for the dead-time calculations from Chapter 8. In Add Back mode, the original activity calculations from the $329 \mathrm{keV}$ photon showed results that were much higher than calculations from the other peaks and the calculations from the $329 \mathrm{keV}$ peak in Singles mode. Upon further investigation, it was shown that the $329 \mathrm{keV}$ photon was being interfered with by ${ }^{143} \mathrm{Ce}$, which has cascading emissions from a $293 \mathrm{keV}$ gamma-ray and a $36 \mathrm{keV}$ X-ray. In Add Back mode, these cascading gamma-rays were summing in to the $329 \mathrm{keV}$ peak, whereas in Singles mode, the summing in was negligible. The correction factor was obtained by using the decay scheme, the efficiency curves, and the $722 \mathrm{keV}$ peak area in each spectrum to determine the amount of interference that contributed to the $329 \mathrm{keV}$ peak. As shown in Figure 87 , all ${ }^{140}$ La activity calculations from Singles mode give very consistent results with the beta counting activity estimation. The downward swing is also observable in the Singles residuals plots for the higher energy photons. 


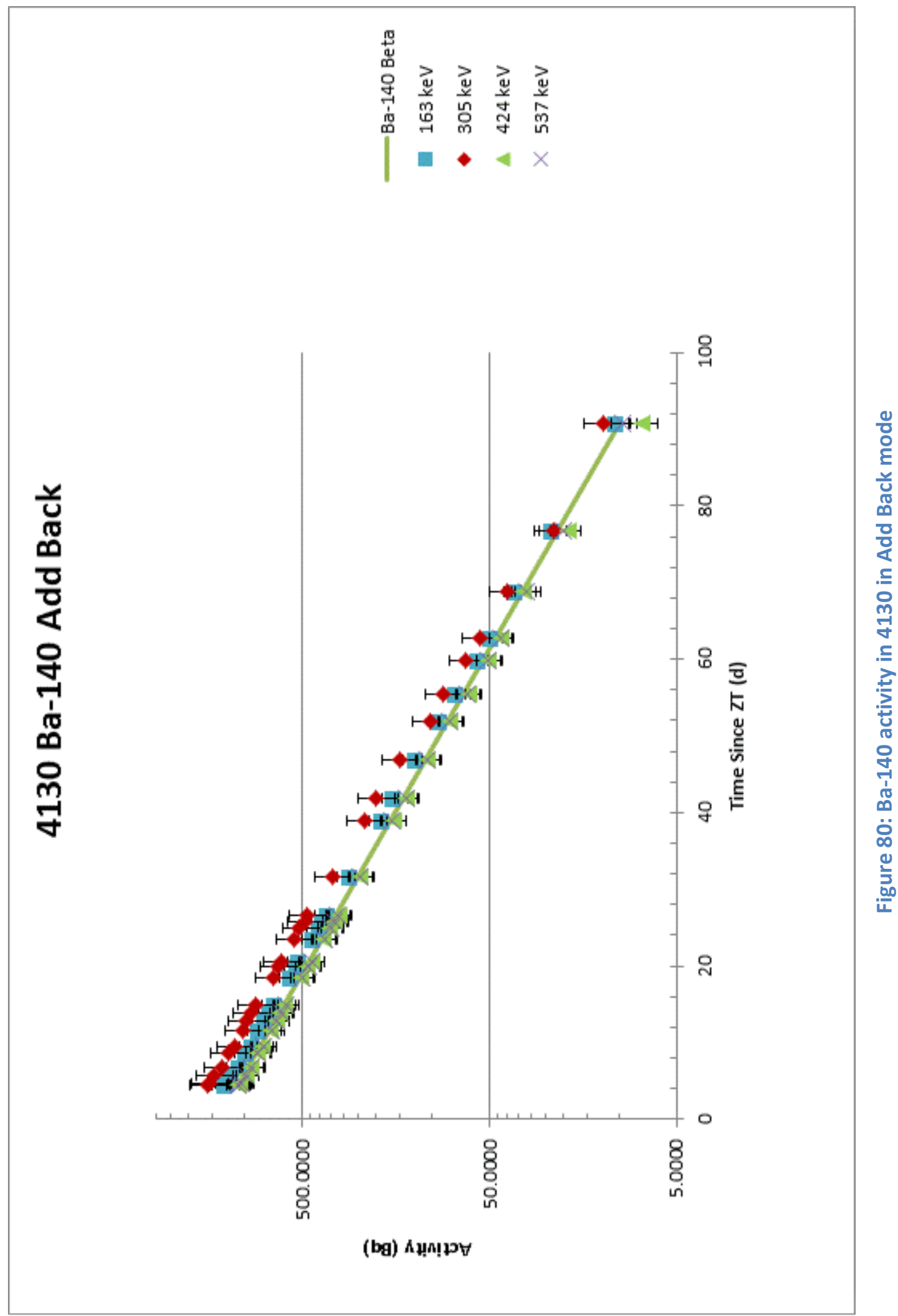




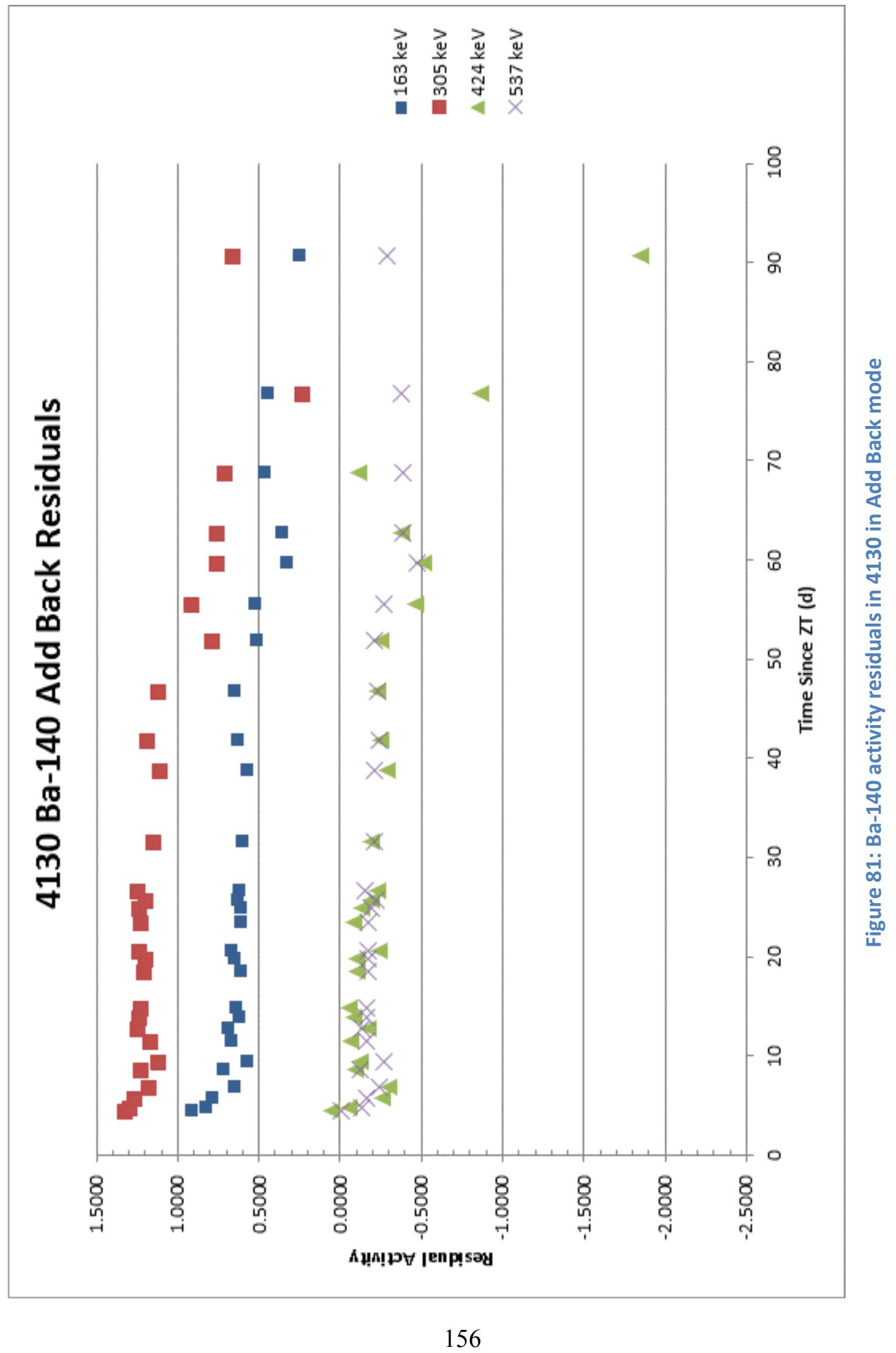




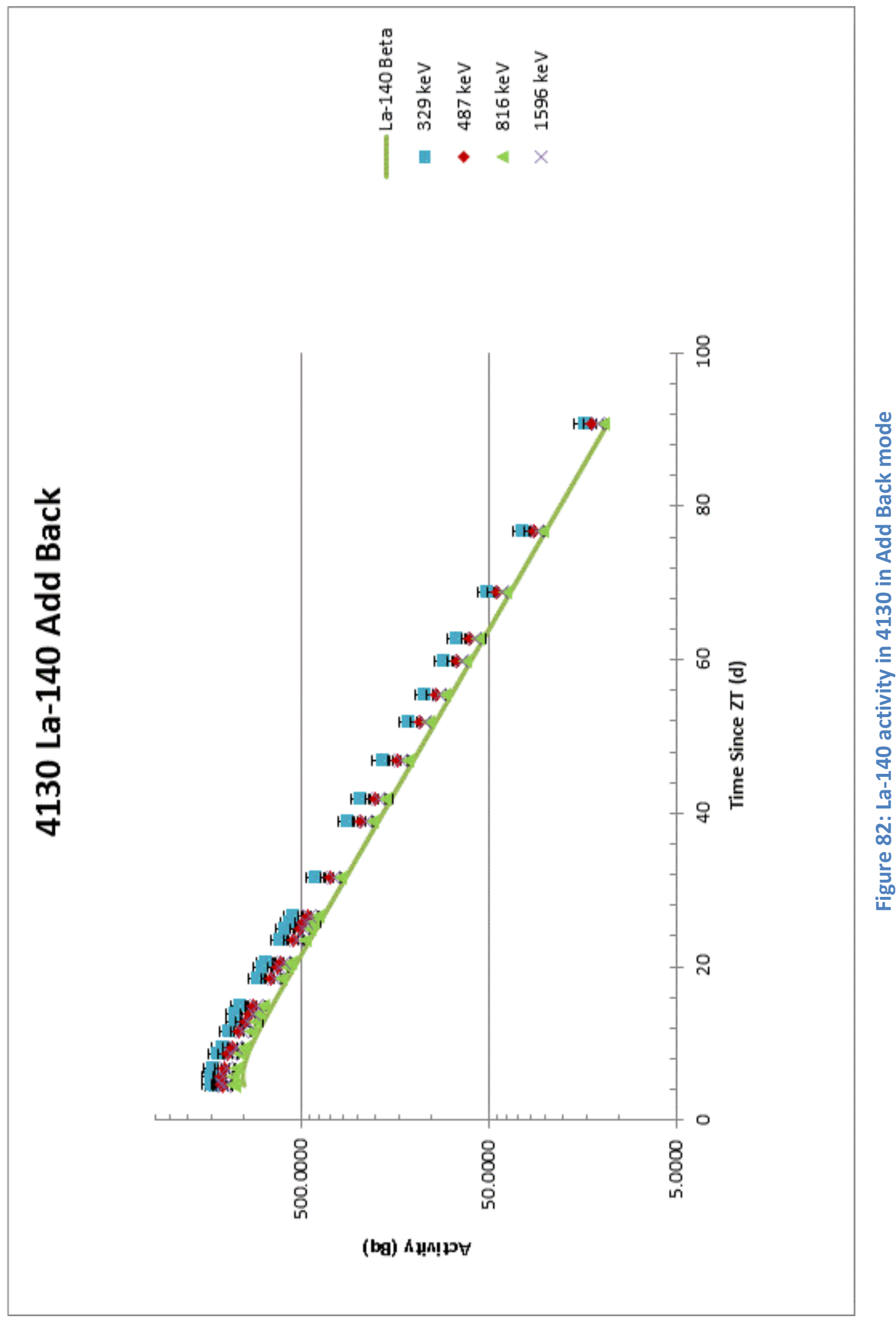




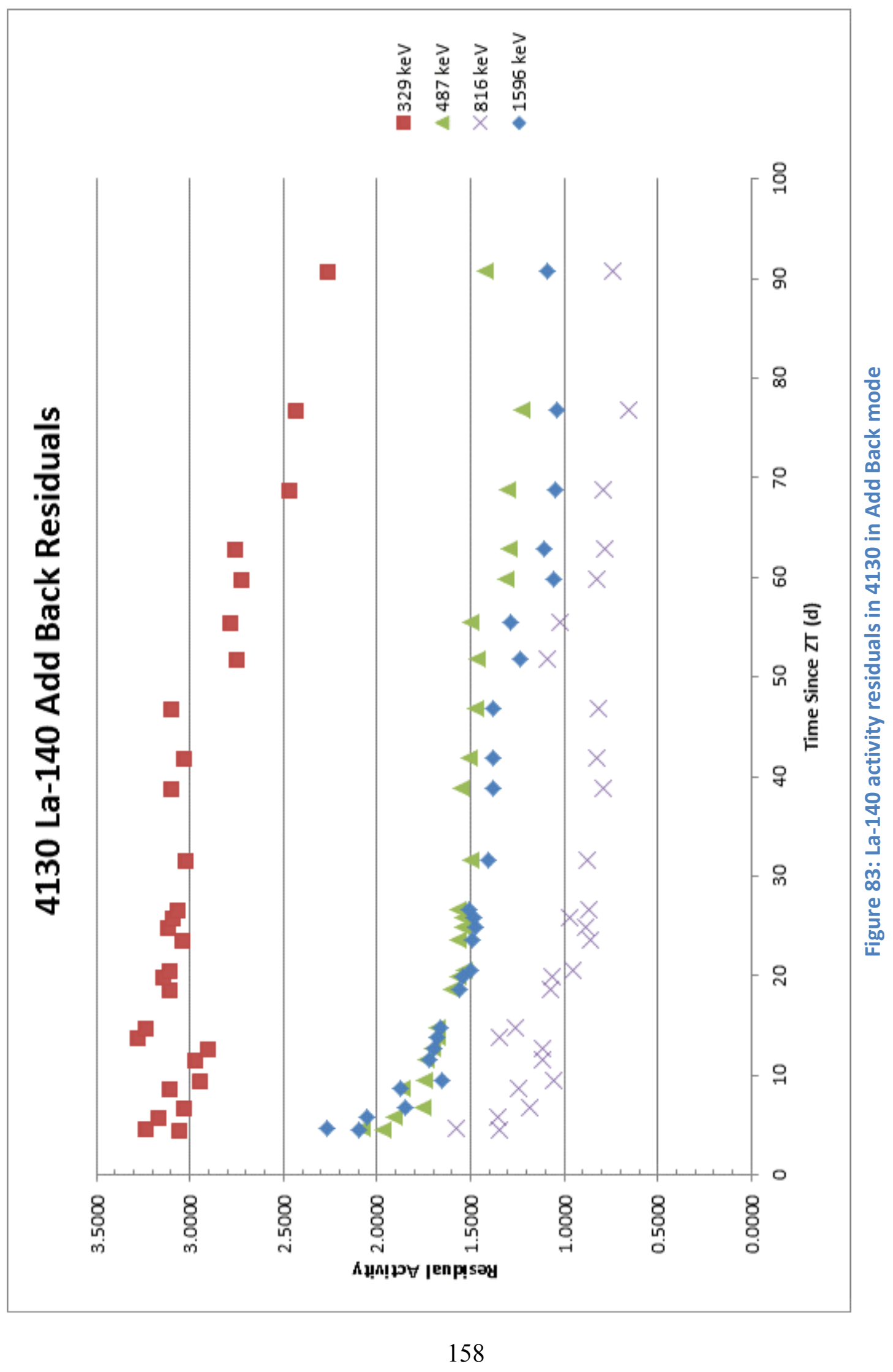




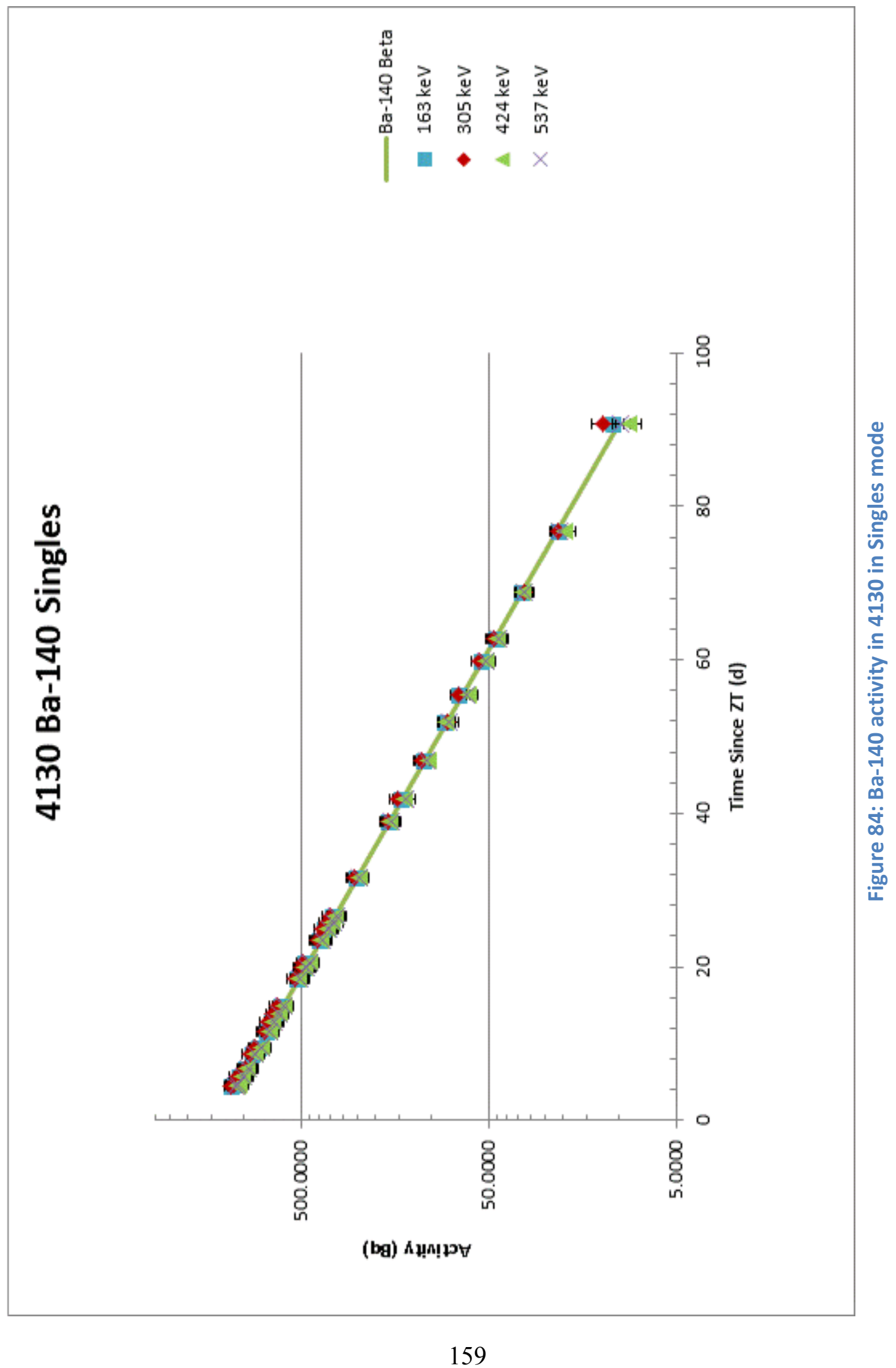




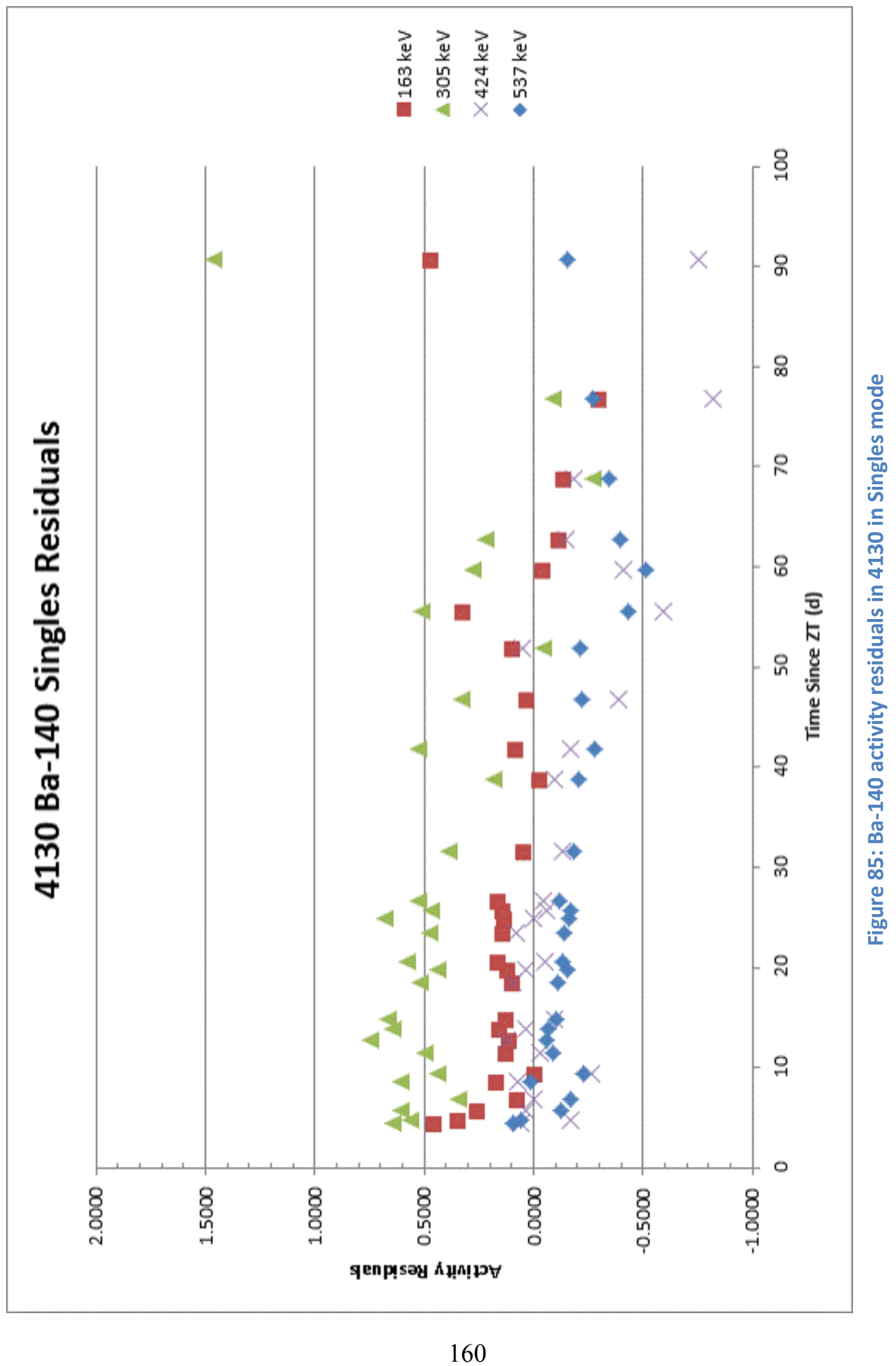




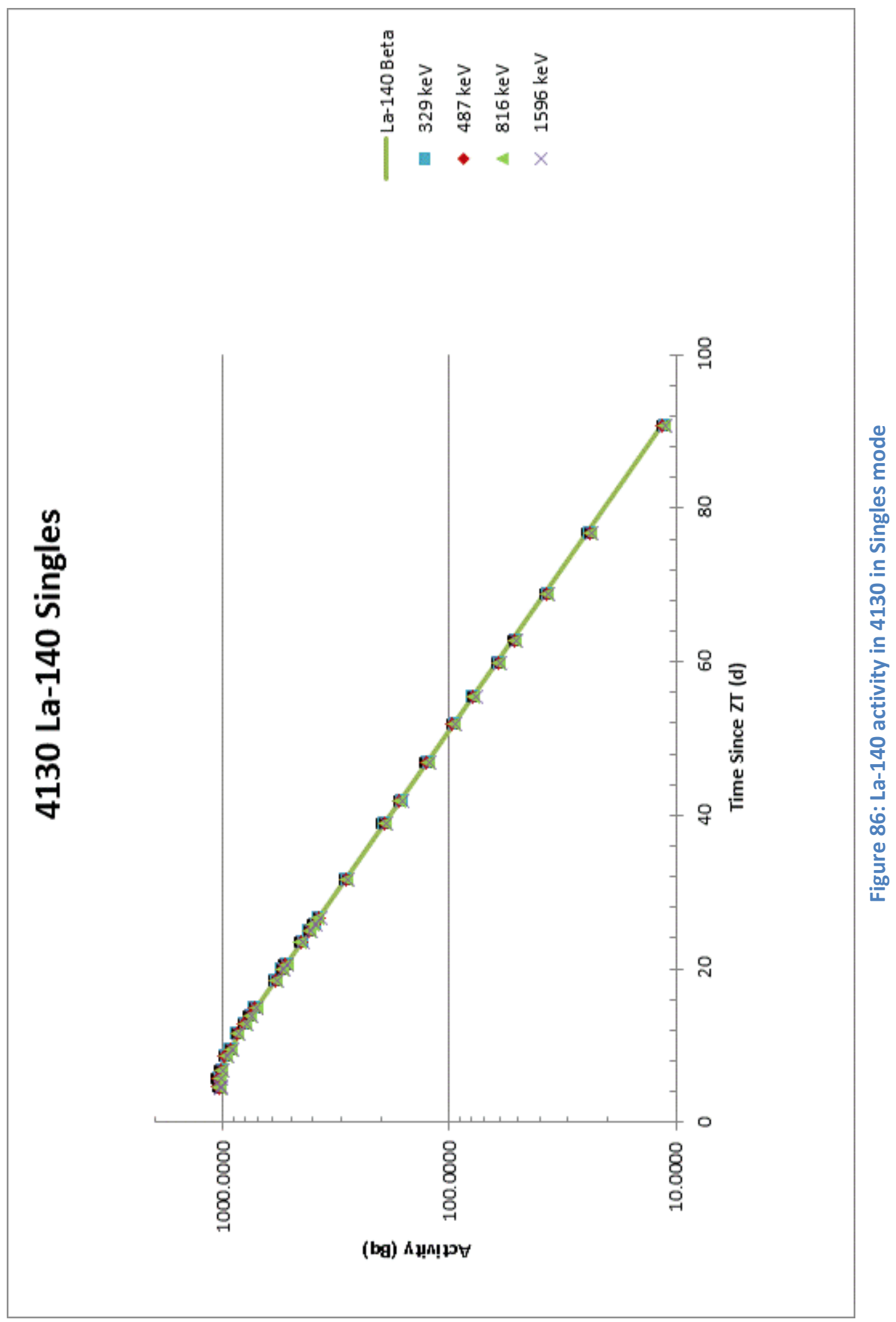




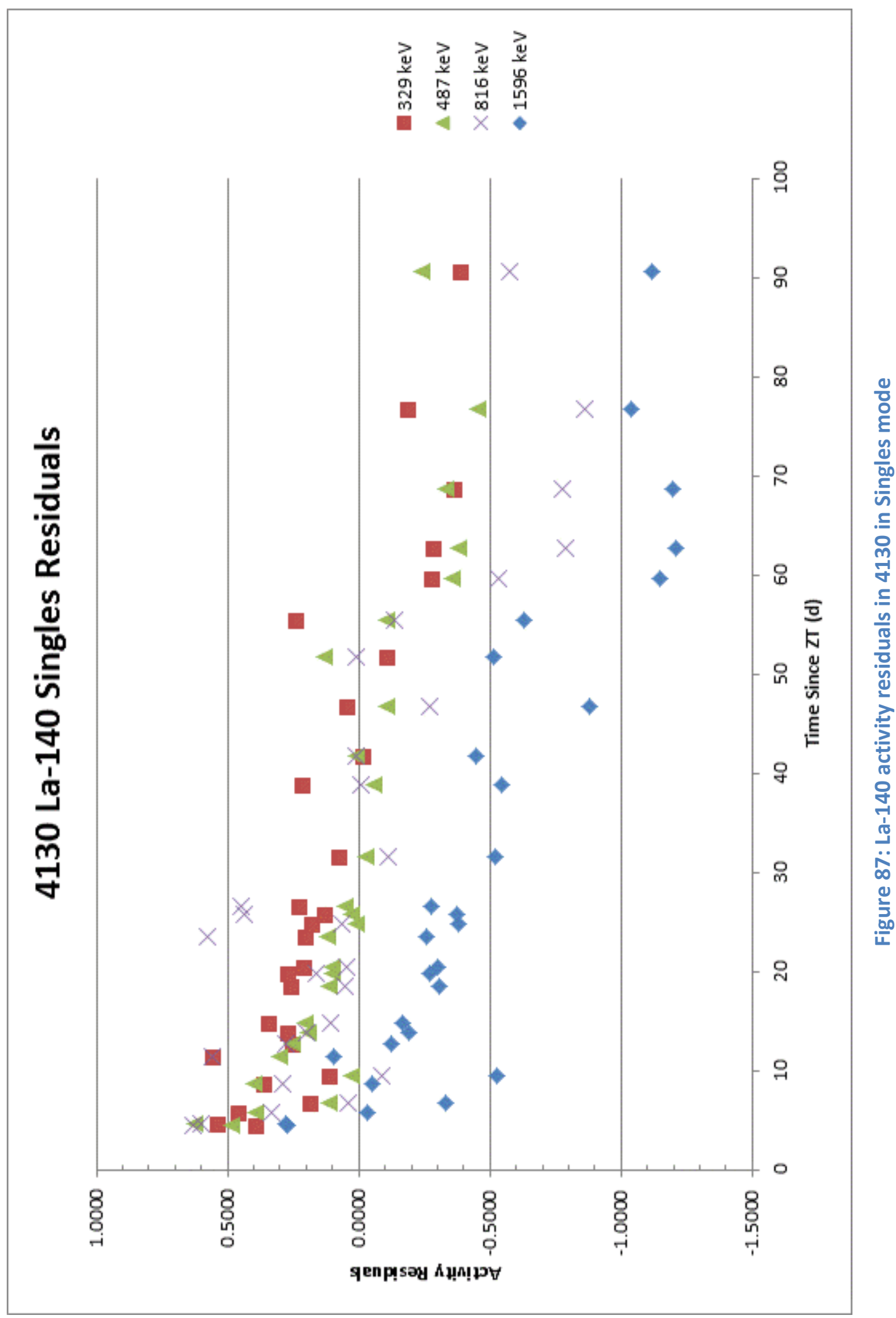


As shown in Figure 89 and Figure 91 , the activity calculations for the ${ }^{99} \mathrm{Mo} /{ }^{99 \mathrm{~m}} \mathrm{Tc}$ fission product pair in Add Back mode were consistent with beta counting estimations when using the $778 \mathrm{keV}$ and $141 \mathrm{keV}$ photons. The $181 \mathrm{keV}$ and $740 \mathrm{keV}$ photons produced results that were high when compared with the beta counting estimations. As shown in Figure 93 and Figure 95, all of the Singles mode activity calculations were comparable with the beta counting estimations. The $778 \mathrm{keV}$ photon shows a downward trend near the end of the counts. 


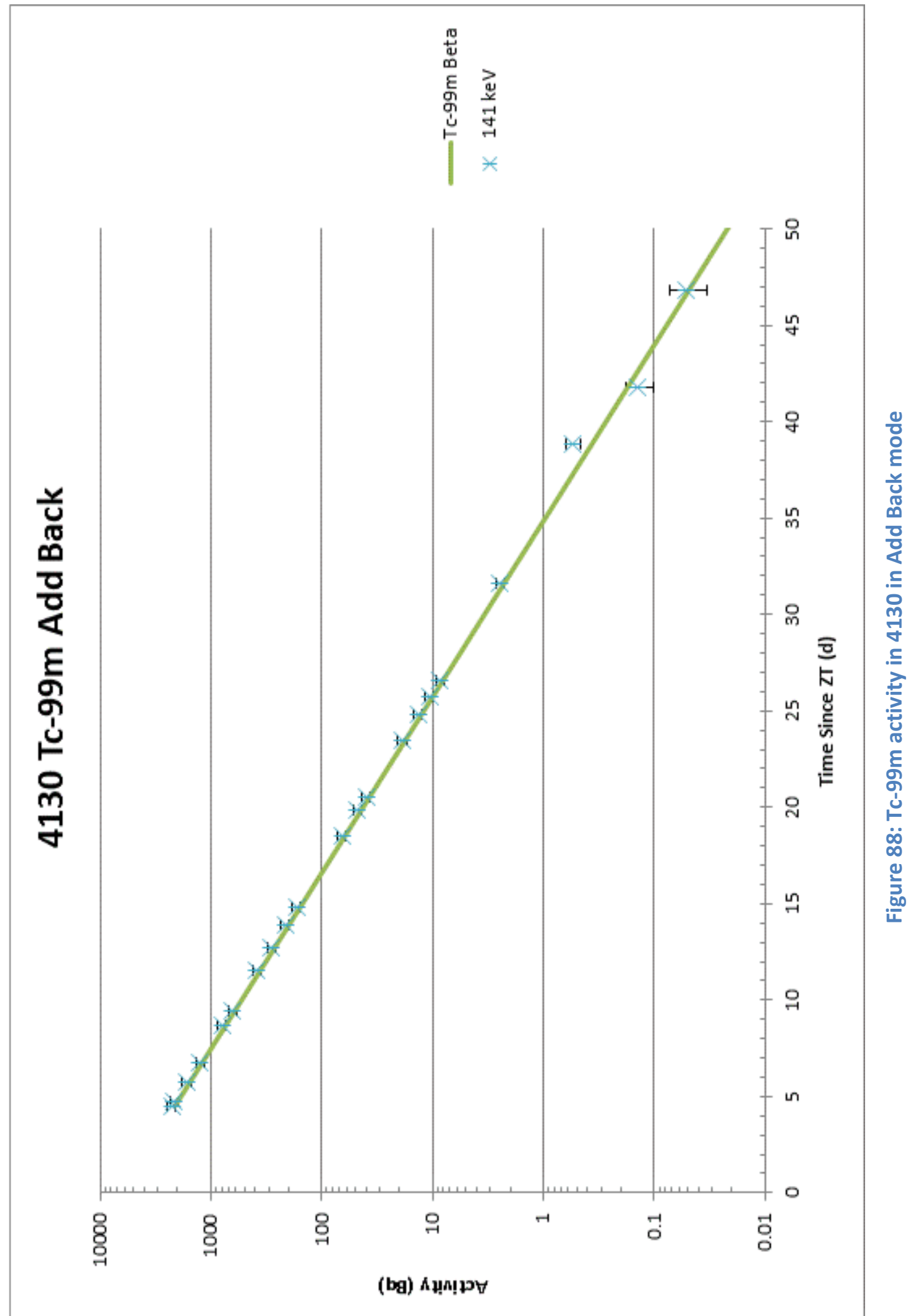




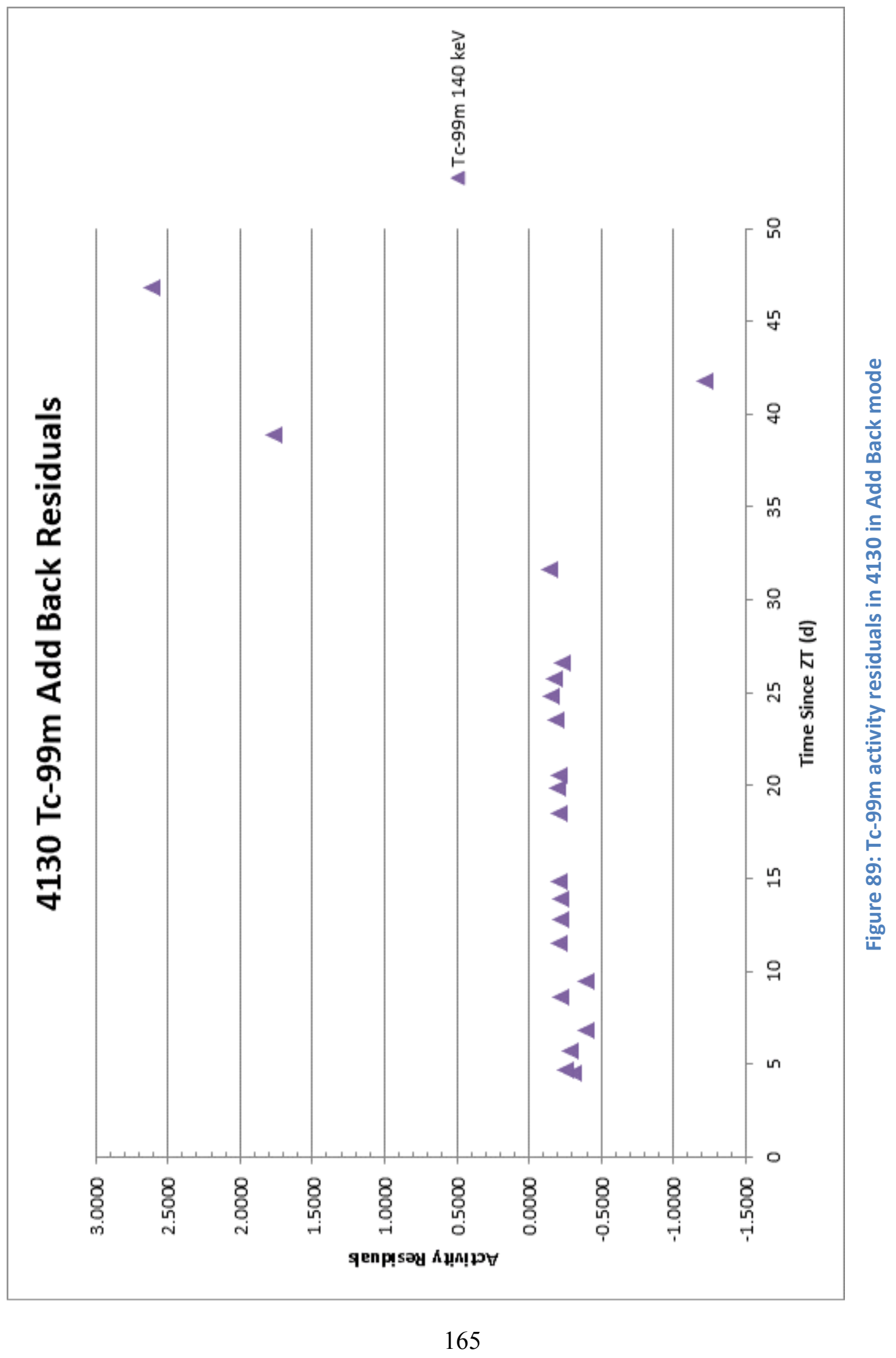




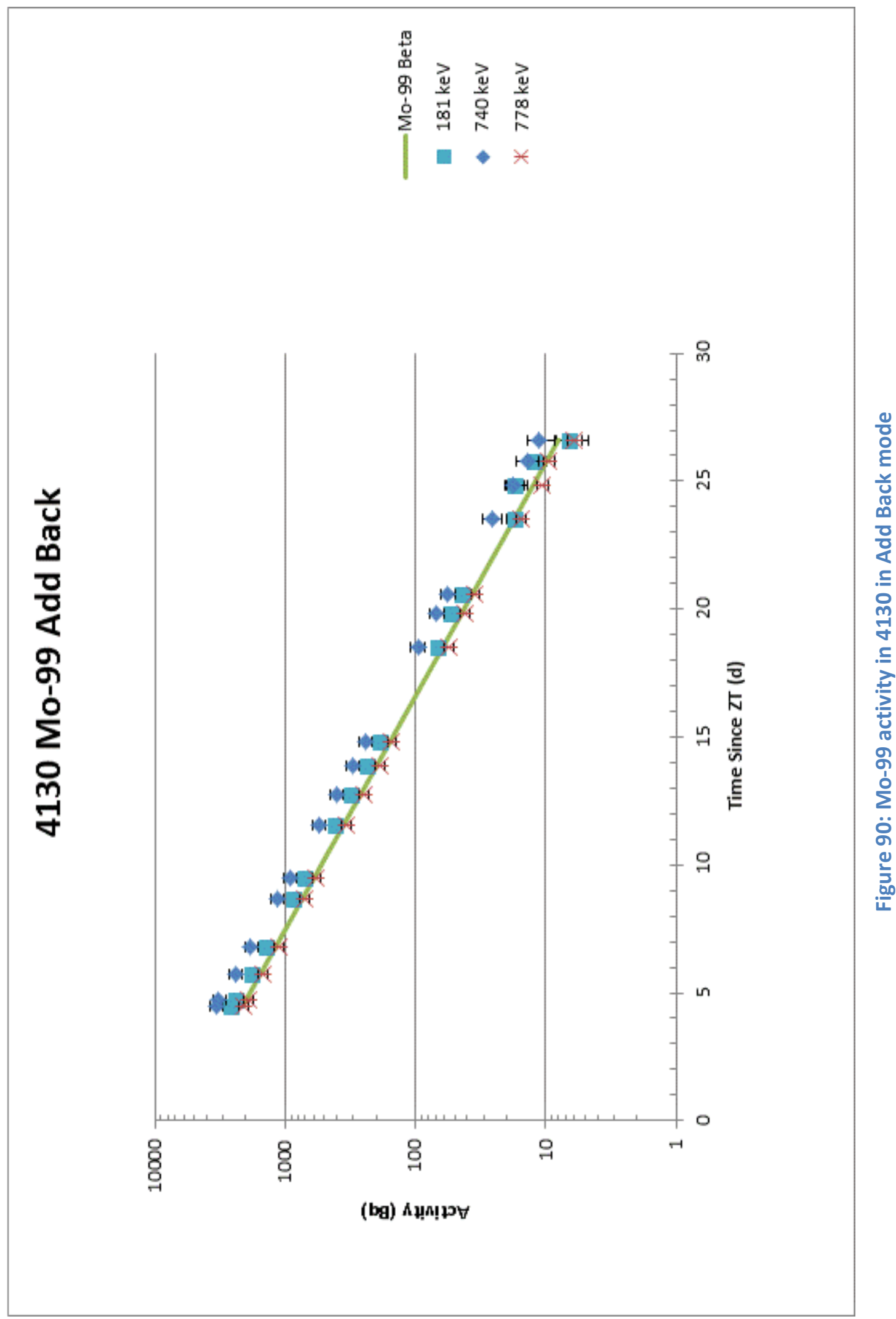




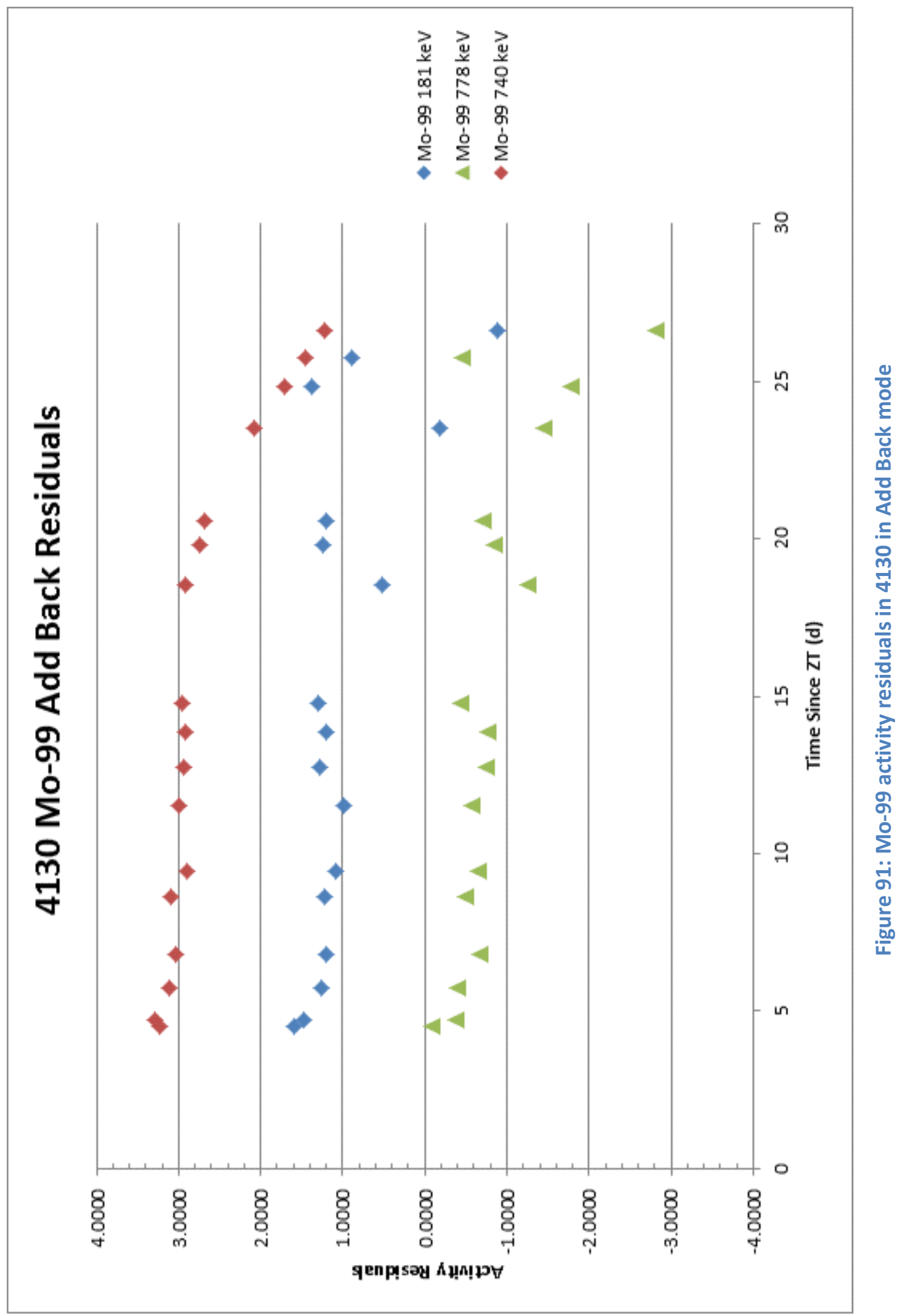




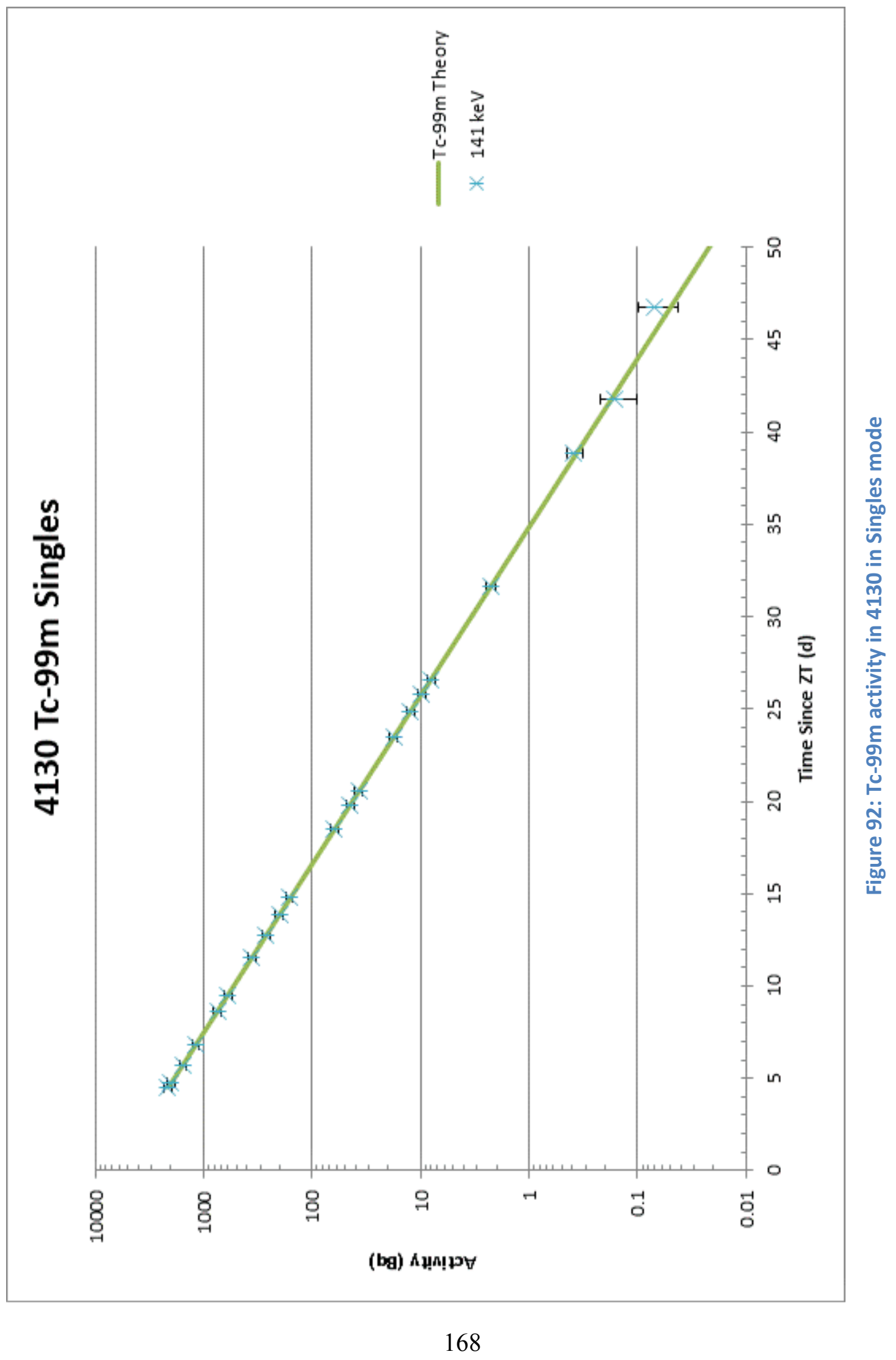




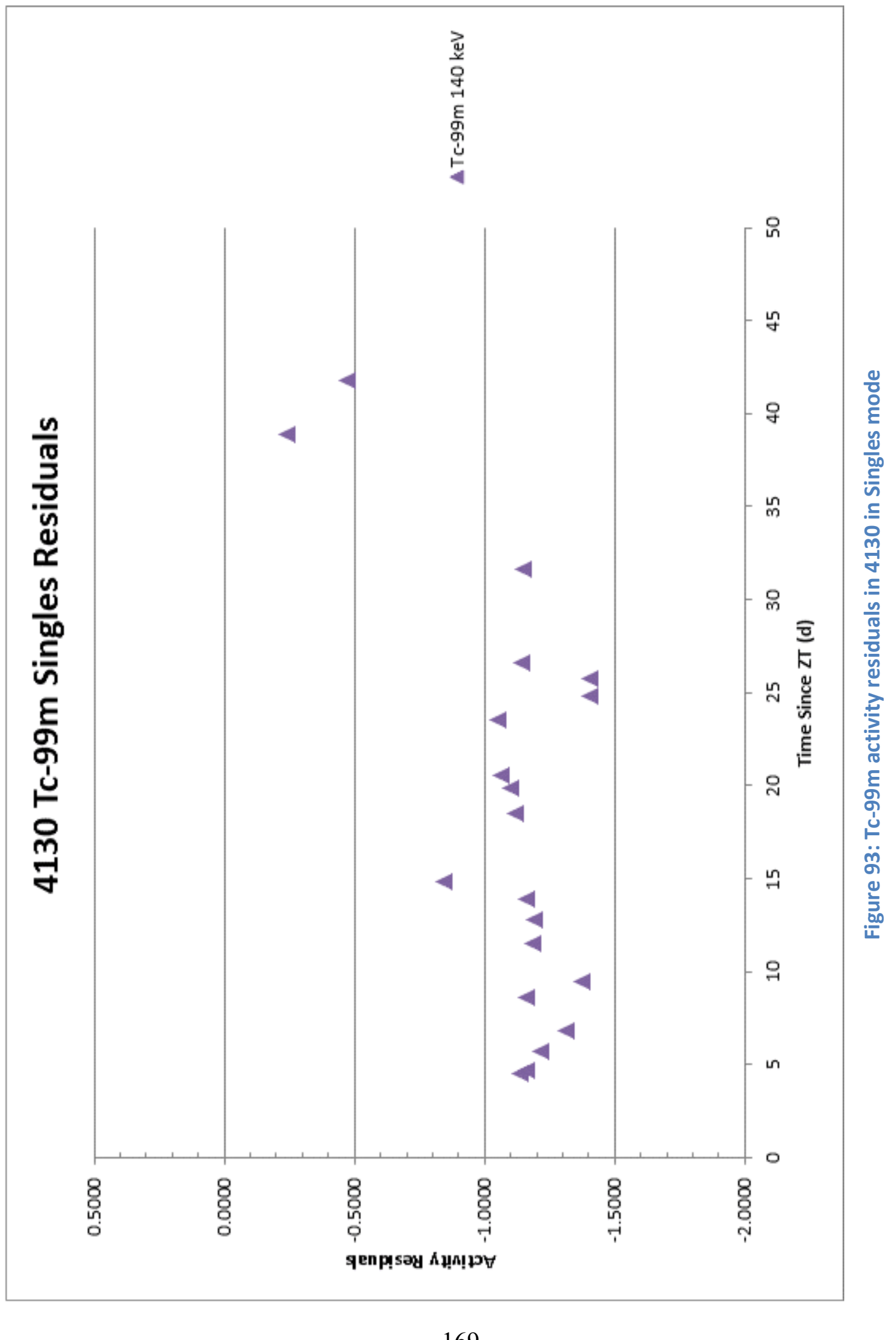




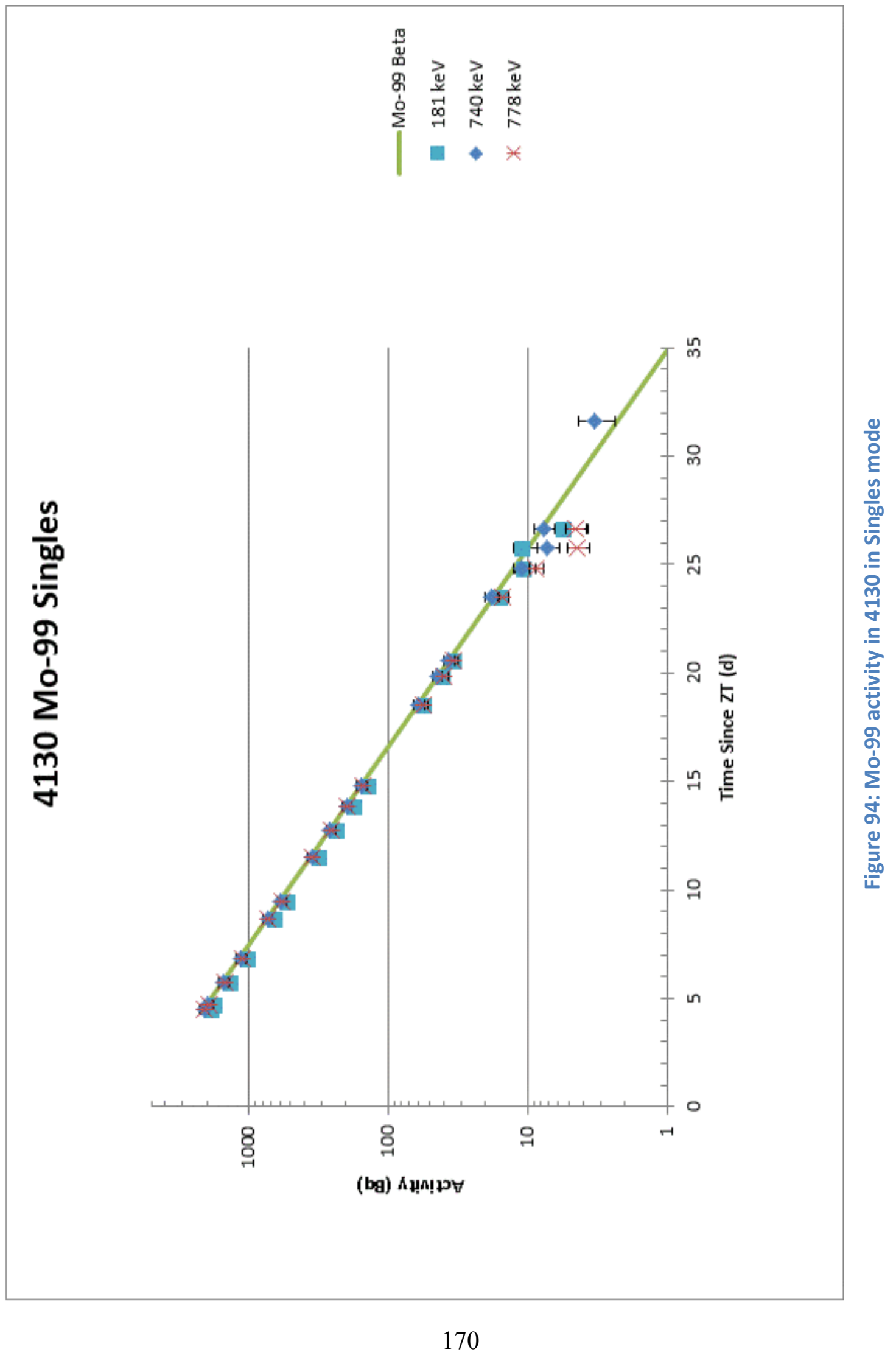




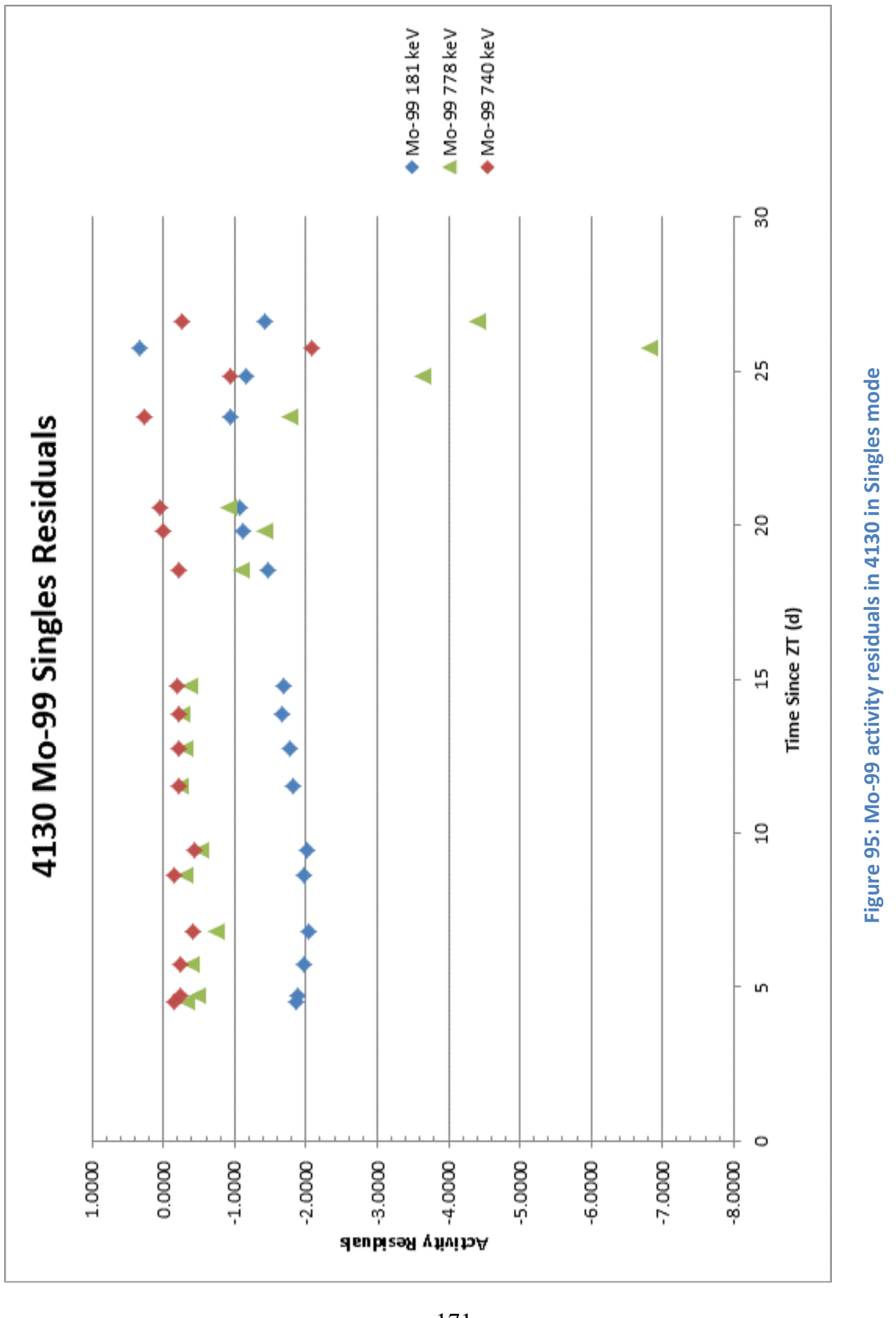


The gamma-gamma coincidence plots for ${ }^{140}$ La seen in Figure 97 and Figure 99 show mixed results. The activity calculations from the coincidence between the $1596 \mathrm{keV}$ photon and the $329 \mathrm{keV}$ photon were high for both gating schemes. The activity calculations for the coincidence between the $1596 \mathrm{keV}$ photon and the $487 \mathrm{keV}$ photon were high for the Kerri gating scheme, but seemed to be accurate for the Misty gating scheme. The activity calculations for the coincidence between the $1596 \mathrm{keV}$ photon and the $816 \mathrm{keV}$ photon were low for the both gating schemes. The activity calculations for the coincidence between the $487 \mathrm{keV}$ photon and the $329 \mathrm{keV}$ photon appeared to be accurate for both gating schemes. Most of the residuals plots showed a downward trend near the beginning of the counts that leveled off very quickly. This is probably due to dead-time inaccuracies due to linear approximations as mentioned above. 


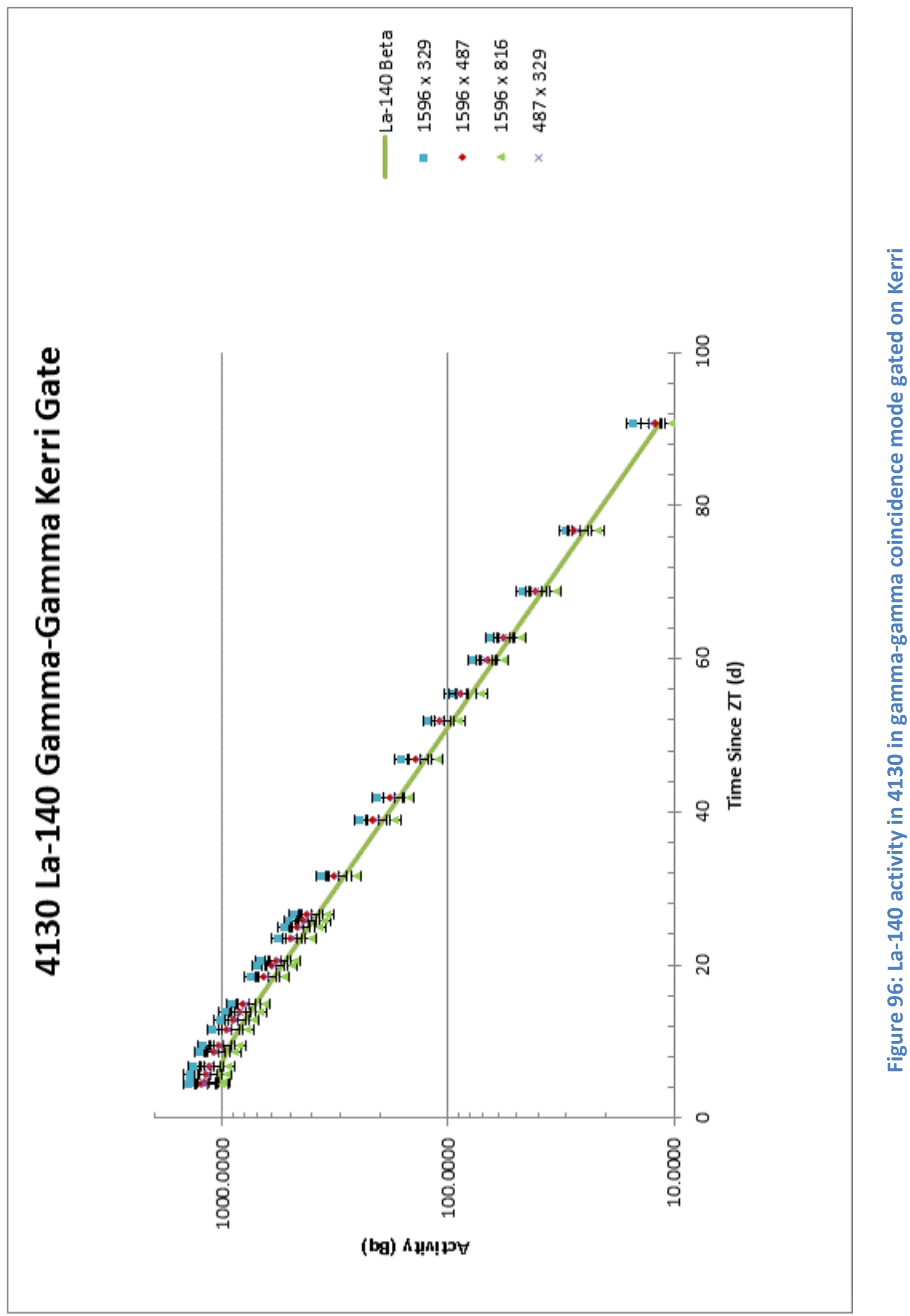




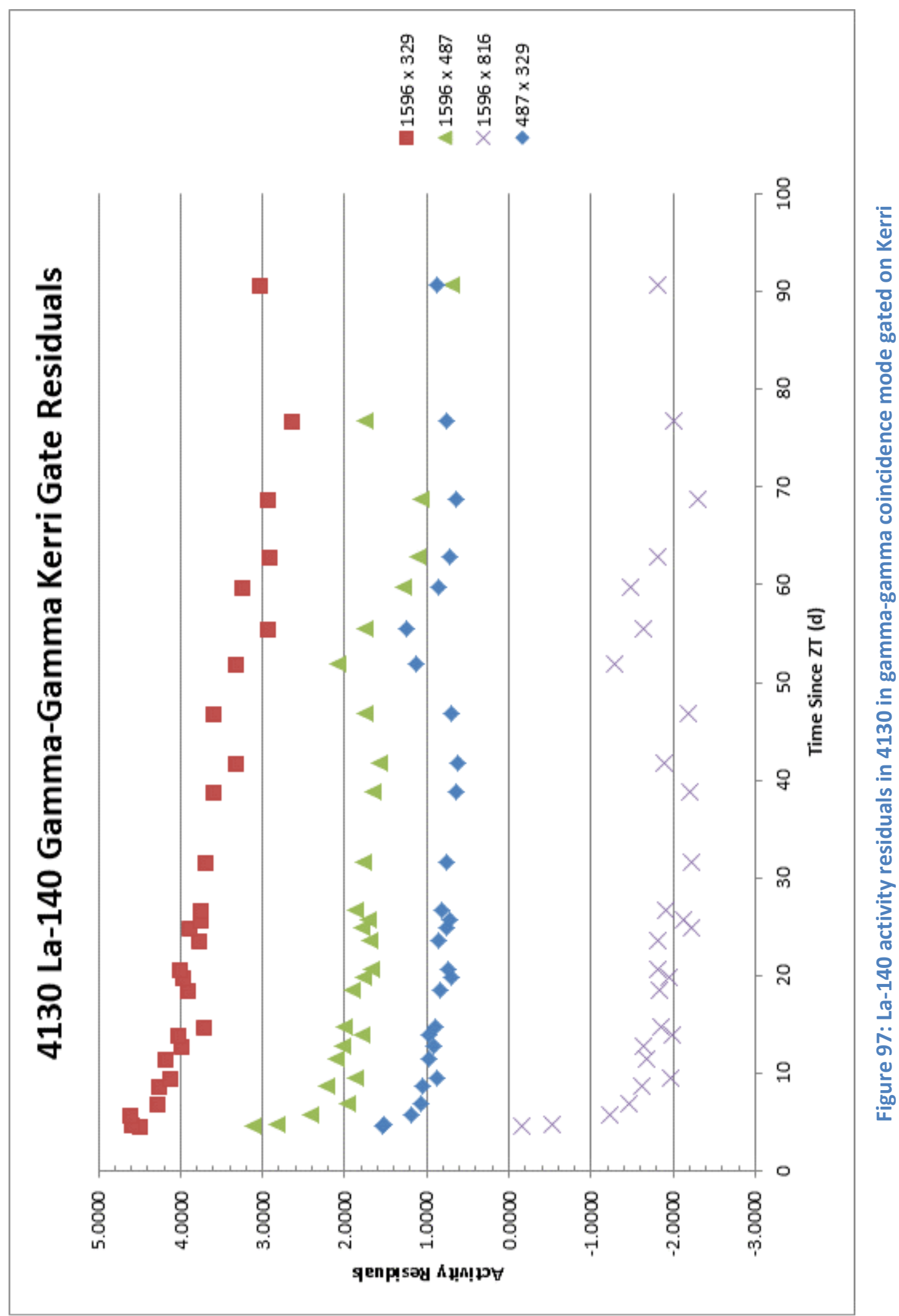




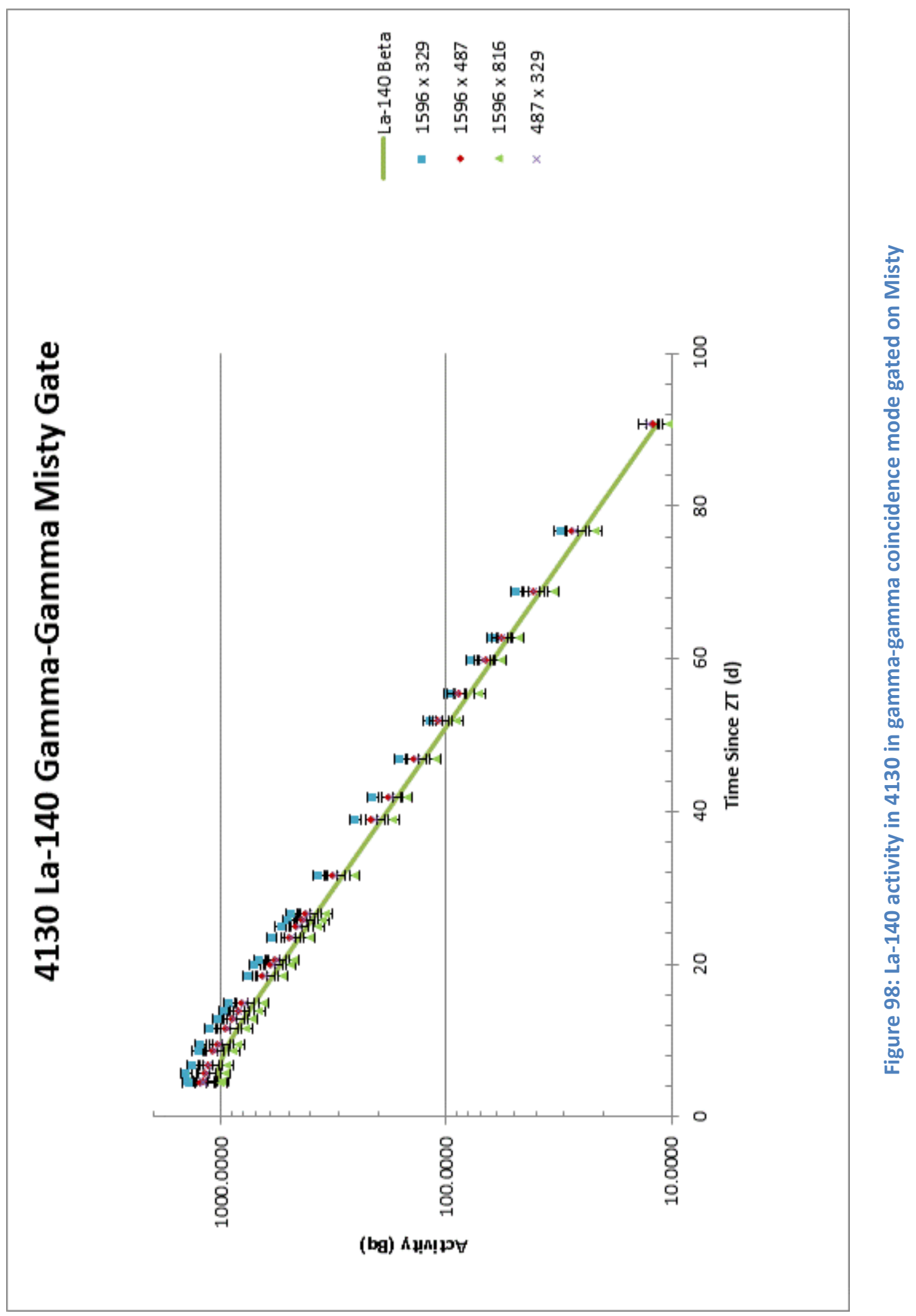




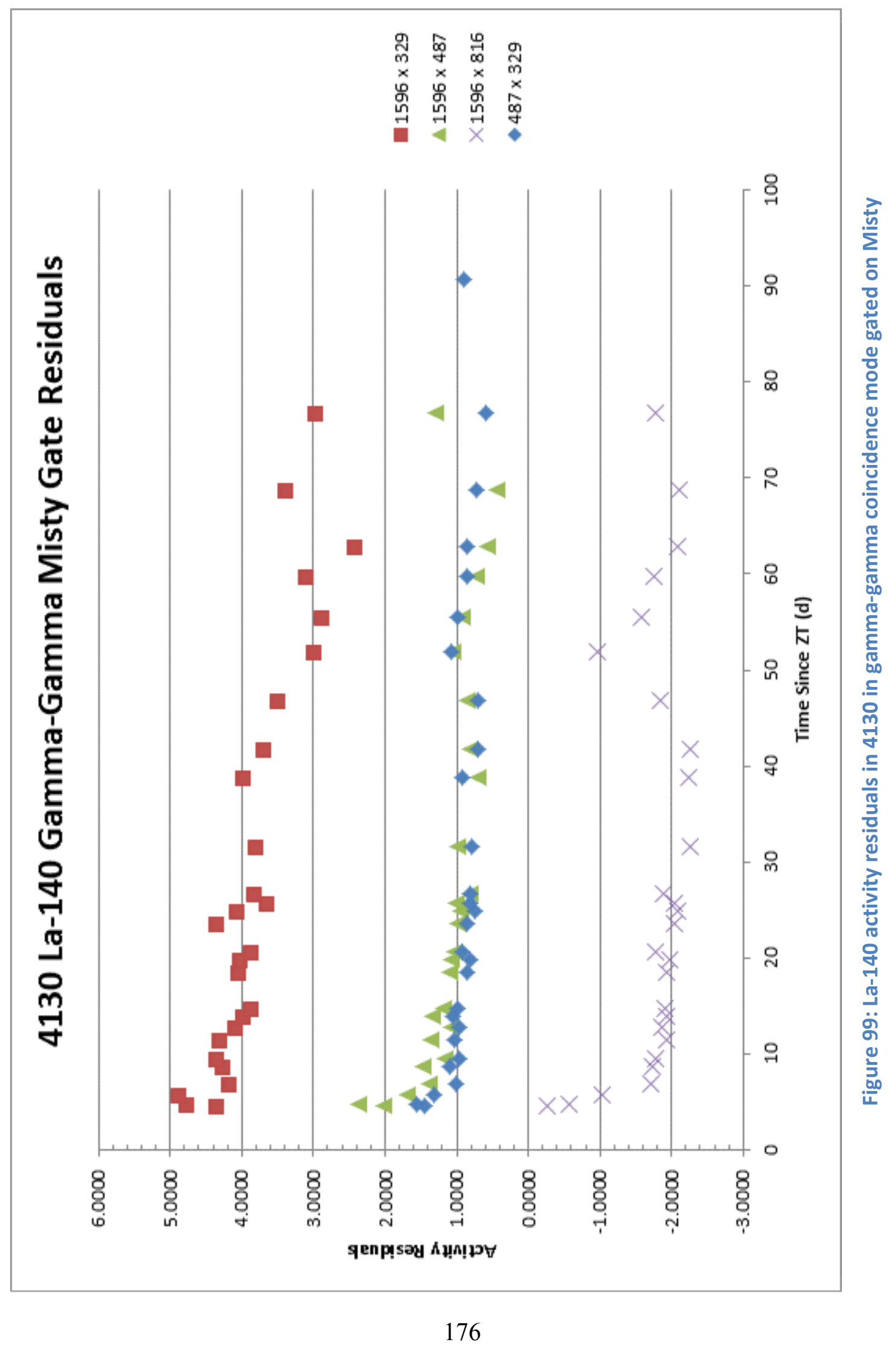


As shown in Figure 101 and Figure 103, the activity calculations for gamma-gamma coincidence mode analyzing ${ }^{140} \mathrm{Ba}$ showed consistent results with the activity estimations from beta counting. These residuals plots show a downward trend near the beginning of the counts that levels off quickly. 


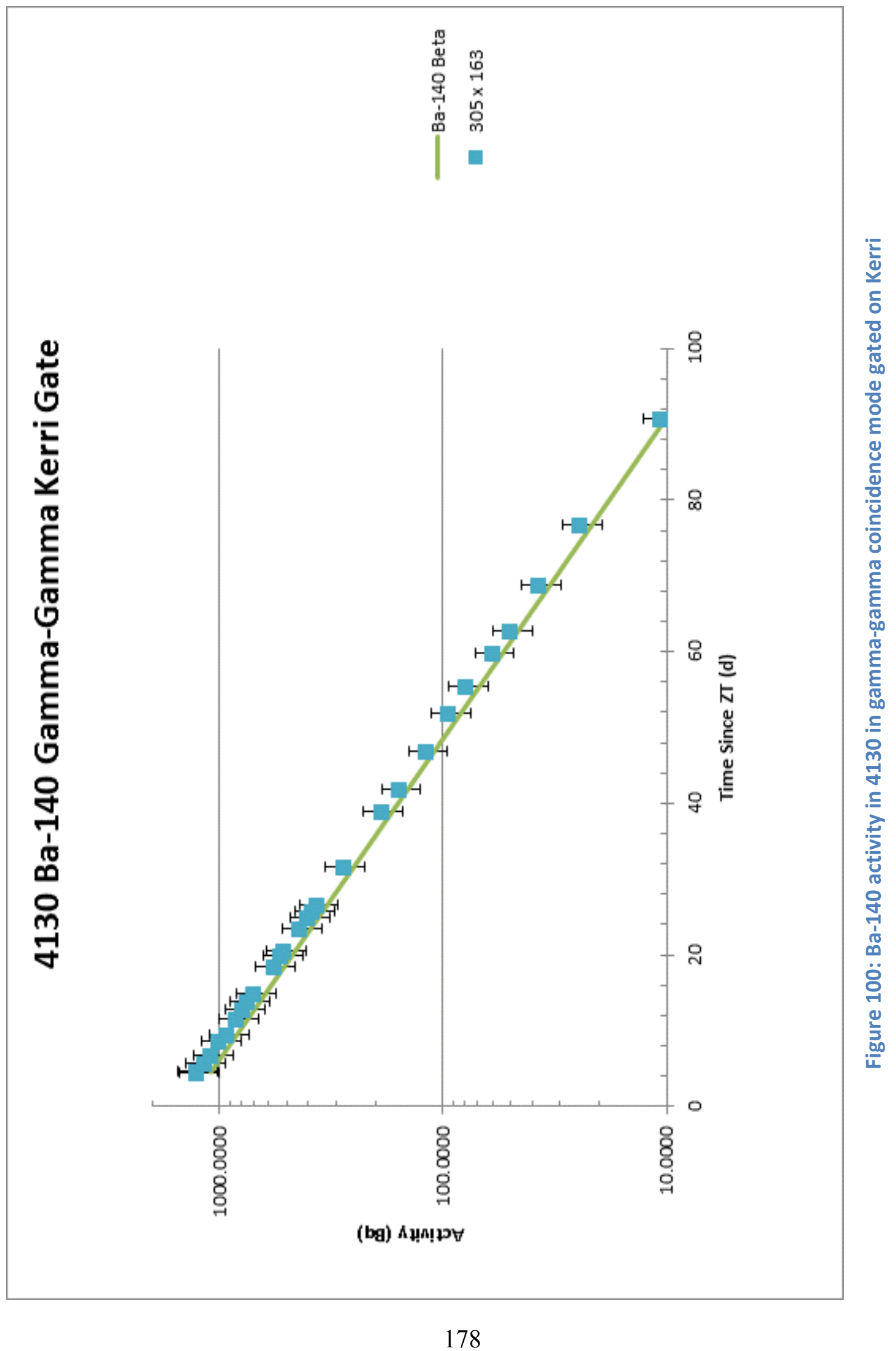




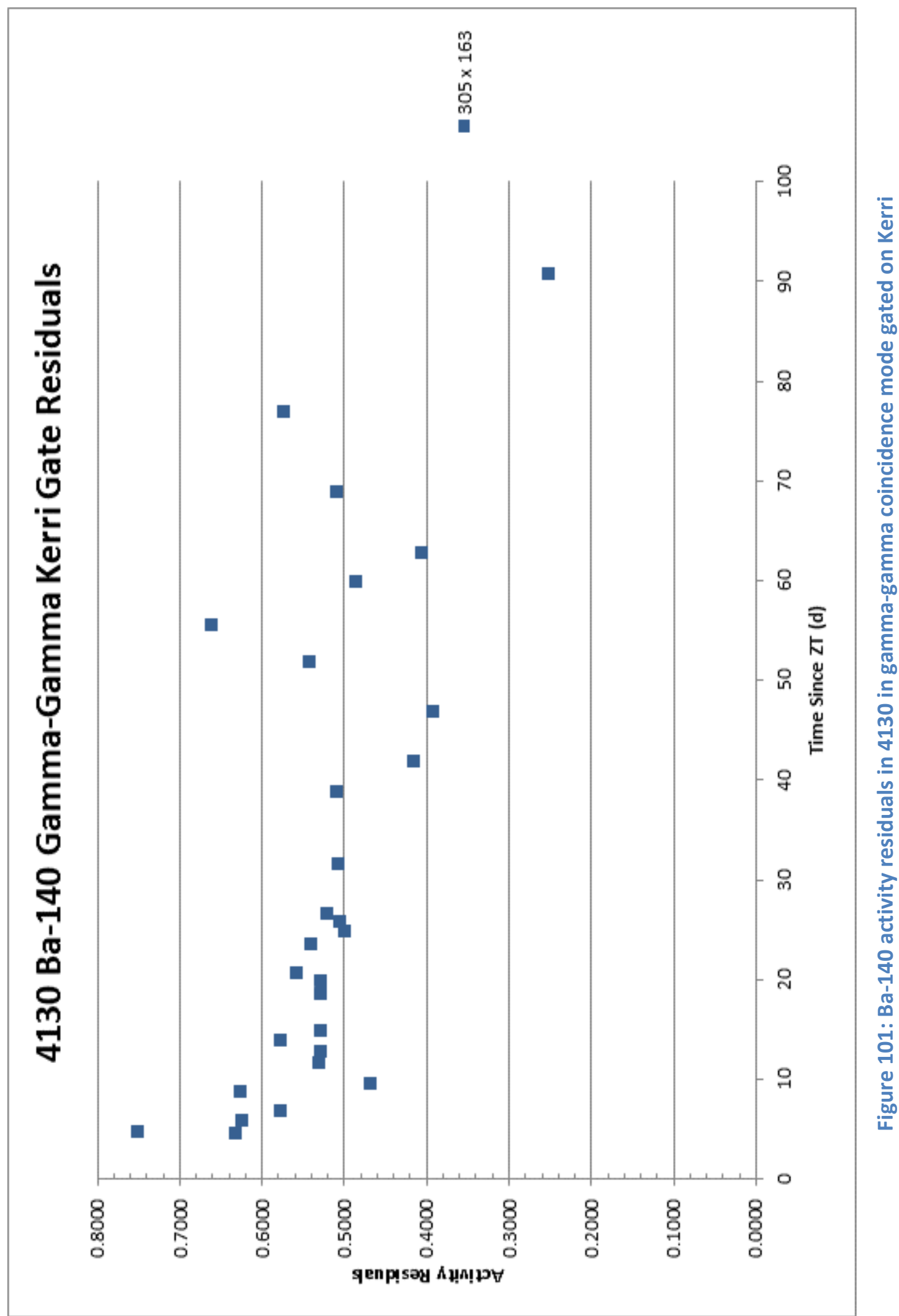




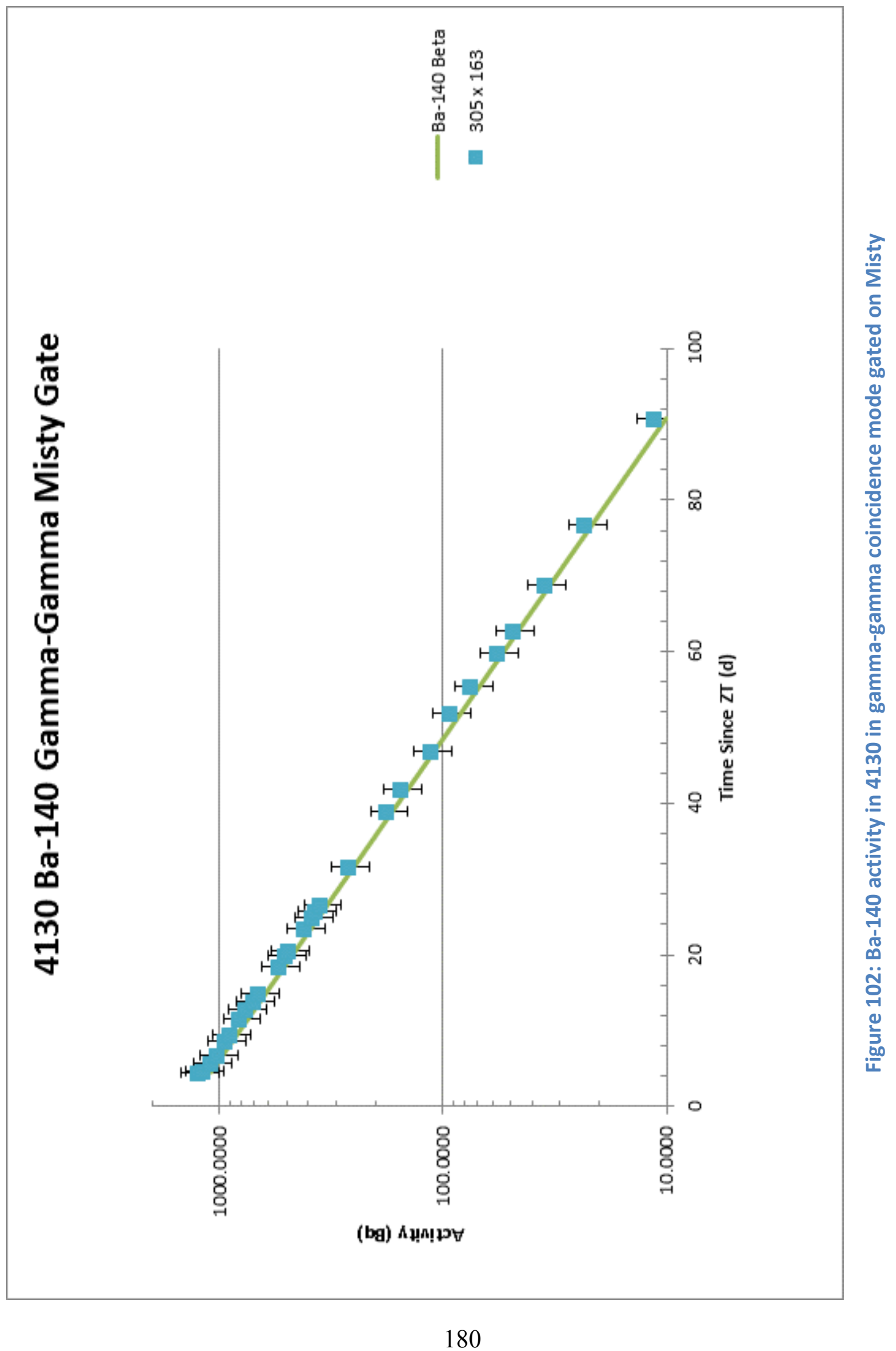




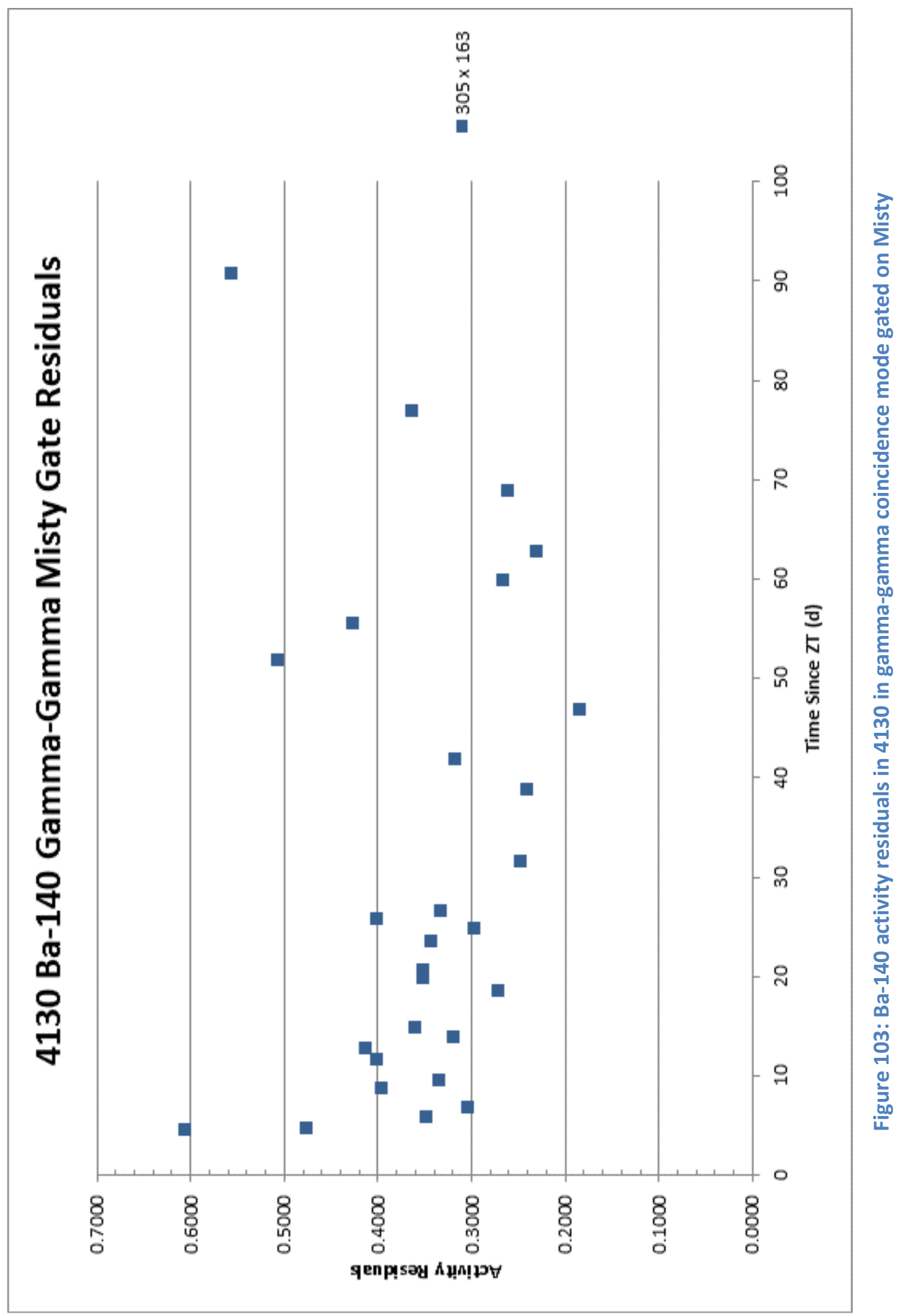


As shown in Figure 105 and Figure 107, the gamma-gamma coincidence activity calculations for ${ }^{99} \mathrm{Mo}$ appear to be consistent with the beta counting estimations. For most of the activity calculations, the residuals fall within $-1 \sigma$ to $1 \sigma$. 


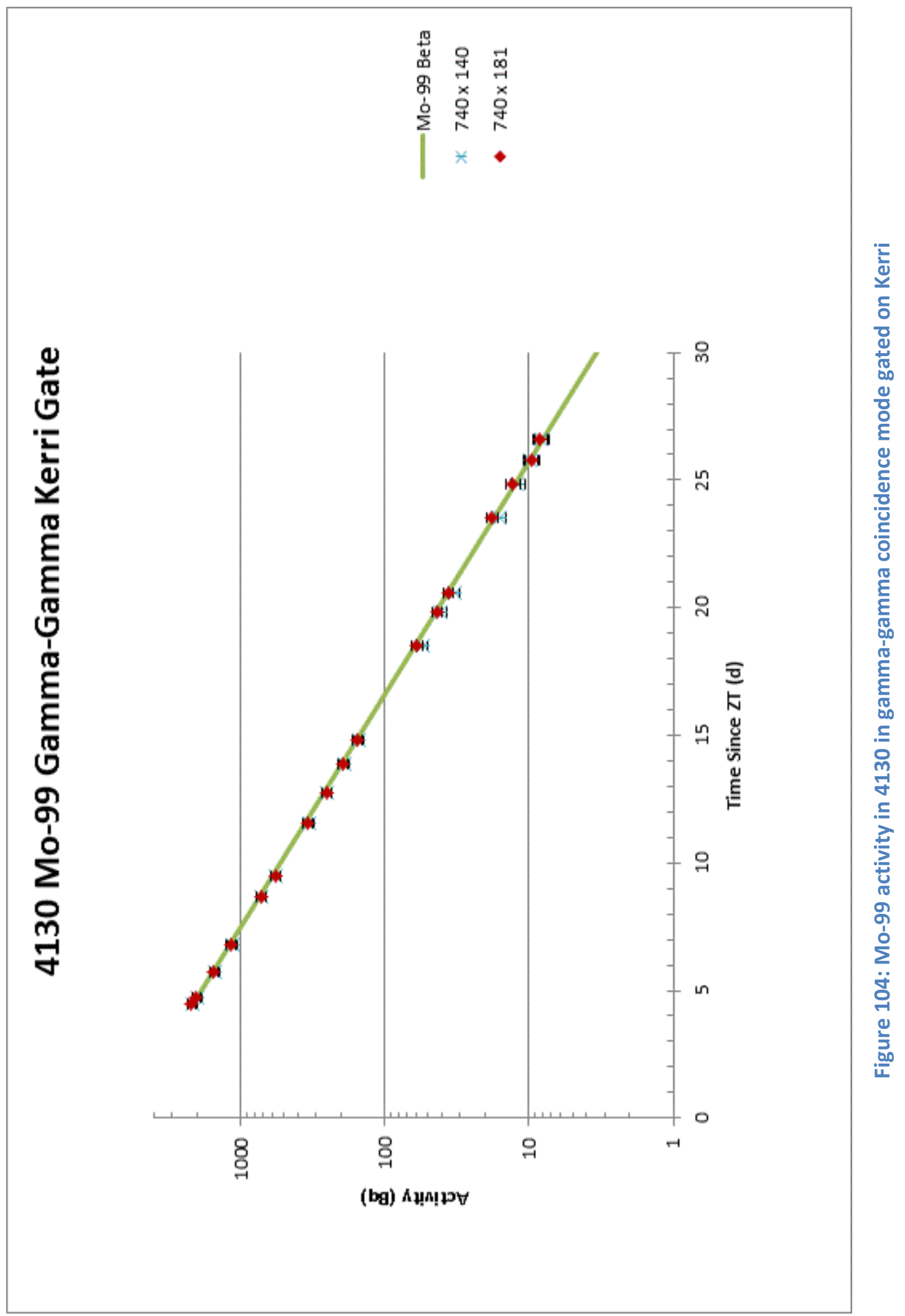




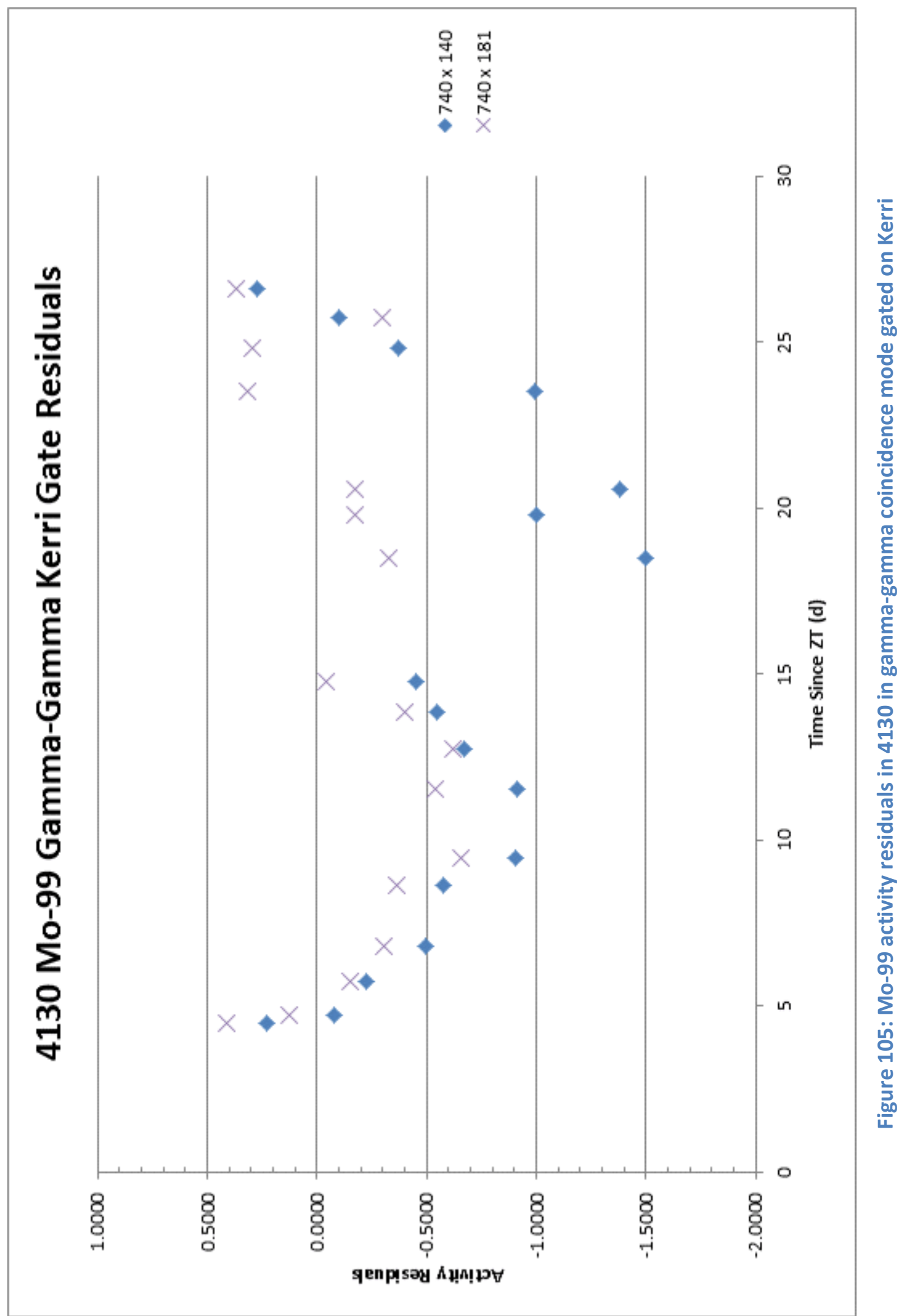




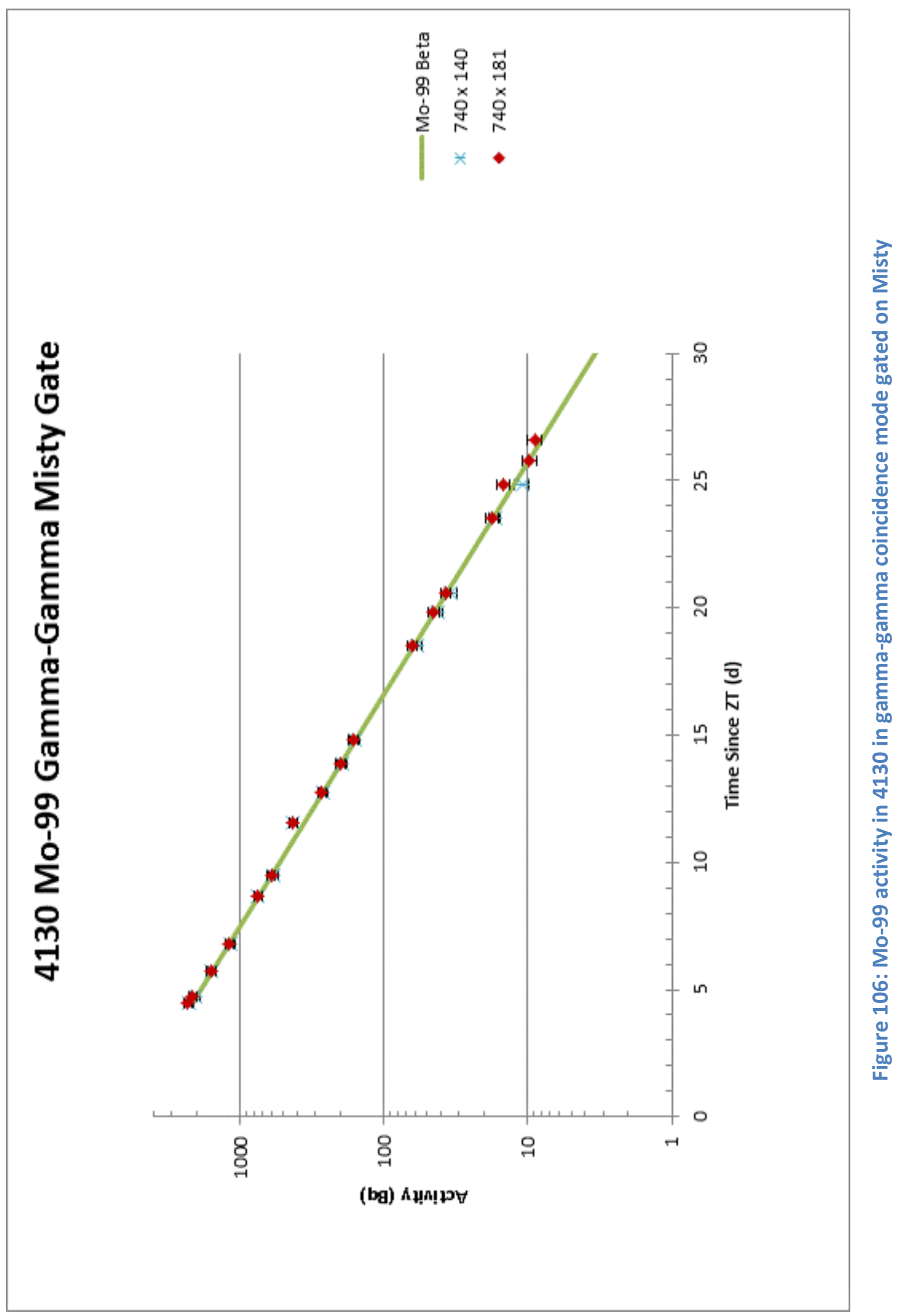




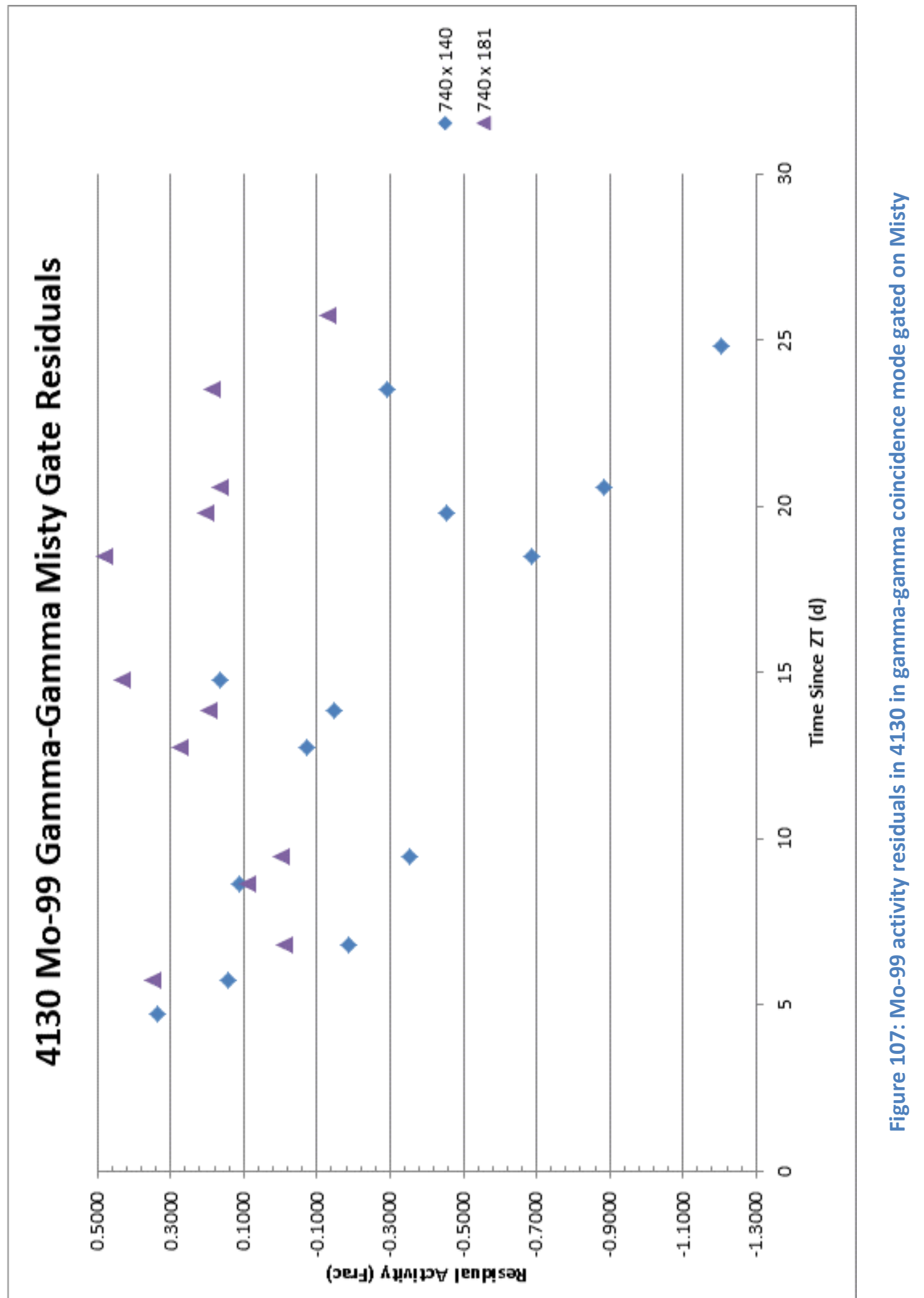




\subsection{Activity Analysis Discussion}

\subsection{1 ${ }^{95} \mathrm{Zr}$ and ${ }^{95} \mathrm{Nb}$}

The ${ }^{95} \mathrm{Zr} /{ }^{95} \mathrm{Nb}$ fission product pair has the longest lifetime out of all the fission product pairs being analyzed in this work. Even at the end of the counts, this parent-daughter system was not observed to go into equilibrium. ${ }^{95} \mathrm{Zr}$, the parent, has a half-life of 64.02 days and ${ }^{95} \mathrm{Nb}$, the daughter, has a half-life of 34.975 days. In observing the ${ }^{95} \mathrm{Zr} /{ }^{95} \mathrm{Nb}$ data in the 4126 and 4130 samples, it appears that the clover system is well-equipped to analyze samples that contain long-lived fission products. In the 4126 sample in Add Back mode, there is a downward trend in the residuals plot, and the points eventually go below $-1 \sigma$. This might be due to inaccurate calculations for the dead-time. The 4126 sample is slightly off-center, which means some crystals will have more of a solid angle than other crystals. The dead-time calculation assumes all crystals have the same solid angle. Dead-time calculations for high-energy photons in Add Back mode are expected to be especially sensitive to sample placement within the system. In the beta counting activity calculations, the activity is found at the time of beta counting and back-calculated to the zero time (end-of-bombardment time and corrected for decay during bombardment).

All the gamma-ray emissions for the ${ }^{95} \mathrm{Zr} /{ }^{95} \mathrm{Nb}$ fission product pair being observed are in the 700-800 keV range. They are also all singlet emissions, so there is no need to correct for TCS. Because of this, it is expected that Add Back mode will have higher peak areas, which may lead to higher precision in the results. Because this fission product pair is being analyzed in a sample with other radionuclides, it may not be the case that Add Back mode will always provide more precise results. This is apparent when analyzing the $724 \mathrm{keV}$ emission from ${ }^{95} \mathrm{Zr}$. ${ }^{143} \mathrm{Ce}$ can interfere with this peak with cascading emissions that sum to $722 \mathrm{keV}(664+58 \mathrm{keV})$ as well as a singlet emission of $722 \mathrm{keV}$. Early in the counts, this peak heavily interfered with the $724 \mathrm{keV}$ peak produced by ${ }^{95} \mathrm{Zr}$, which caused errors in the peak fitting techniques. The Add Back and Singles spectra in the $724 \mathrm{keV}$ area are shown in Figure 108 and Figure 109. As can be seen, the Add Back spectrum has a slight hump near the low end and an obvious peak near the high end. The slight hump was being included in the $724 \mathrm{keV}$ peak fit, giving an inflated area. The Singles mode spectrum also has a low energy hump, but it appears to be more pronounced. The fitting 
software did not have issues in the Singles spectra. To correct for this, an interactive peak fitting was used to distinguish the $722 \mathrm{keV}$ peak from the $724 \mathrm{keV}$ peak.

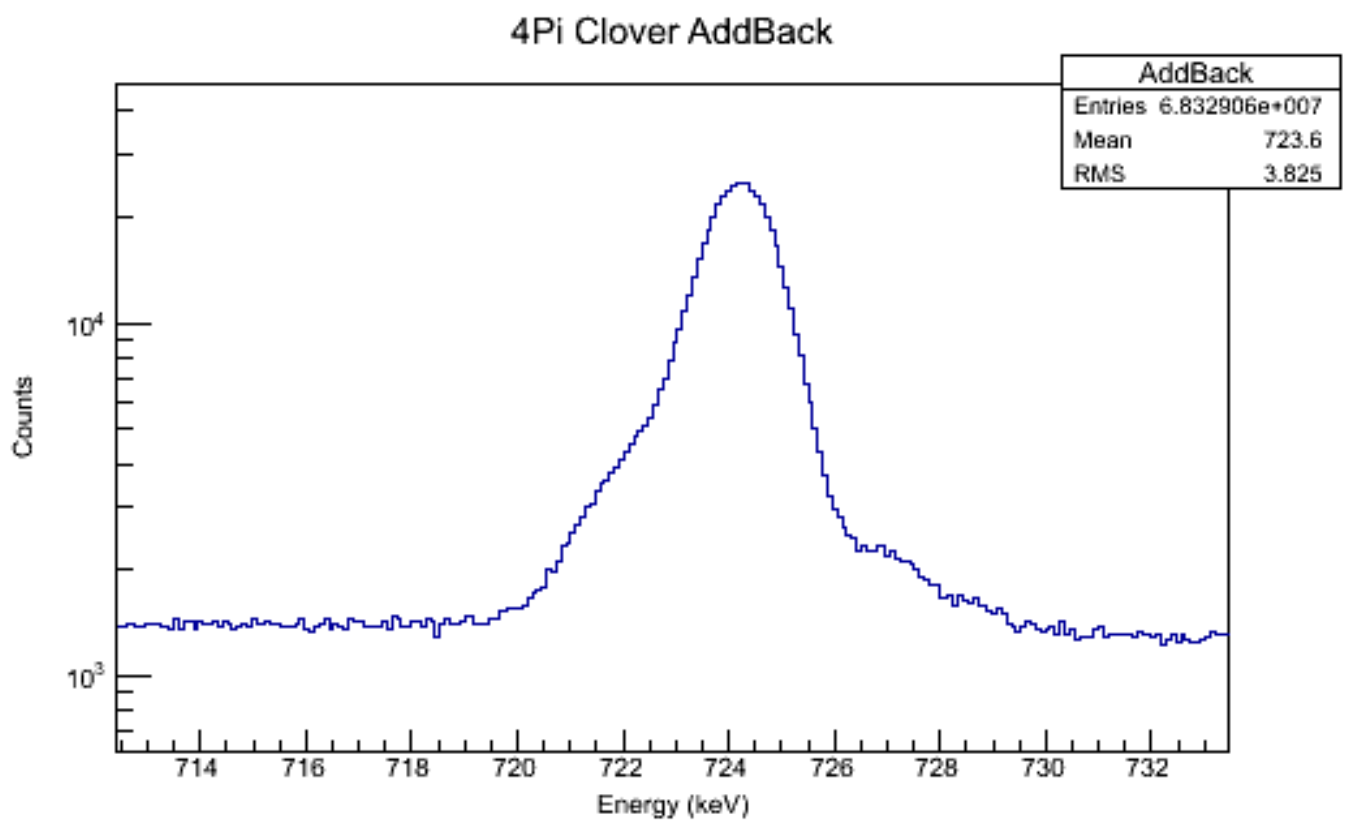

Figure 108: 4130 Add Back spectrum 4.5 days after zero time.

The $724 \mathrm{keV}$ peak is interfered with by at least two other peaks.

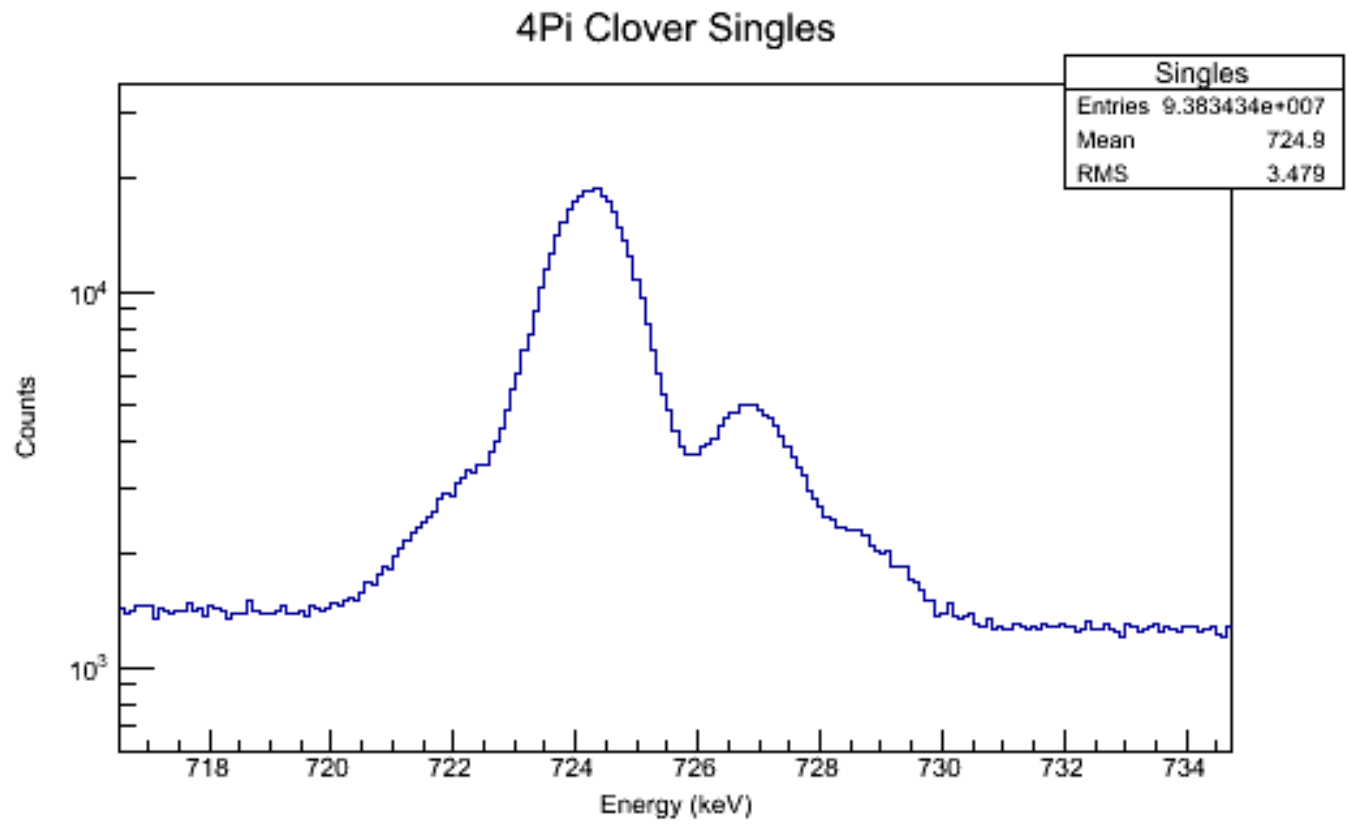

Figure 109: 4130 Singles spectrum 4.5 days after zero time.

The peaks interfering with the $\mathbf{7 2 4}$ keV peak are more discernible in Singles mode. 
Because of interferences and the magnitude of the underlying continuum, the best counting mode to use for a specific nuclide may not be obvious. Despite this, without really understanding all of the interferences involved, the expected number of counts in the peak per decay of the nuclide can indicate the best counting mode to use. The expected number of counts in each peak per decay of the nuclide is found from the $\mathbf{S}$ matrix, obtained by the procedure outlined in Chapter 9. Table 17 shows the difference between the expected counts per decay of each gamma-ray emission in Add Back and Singles modes.

Table 17: Comparison of Add Back and Singles modes in detecting Zr-95/Nb-95

\begin{tabular}{|c|c|c|}
\hline Energy in keV (Nuclide) & $\begin{array}{c}\text { Add Back (Counts in Peak per } \\
\text { Decay) }\end{array}$ & $\begin{array}{c}\text { Singles (Counts in Peak per } \\
\text { Decay) }\end{array}$ \\
\hline $724\left({ }^{95} \mathrm{Zr}\right)$ & $0.0845 \pm 0.0016(2 \%)$ & $0.0555 \pm 0.0011(2 \%)$ \\
\hline $756\left({ }^{95} \mathrm{Zr}\right)$ & $0.1009 \pm 0.0019(2 \%)$ & $0.0657 \pm 0.0013(2 \%)$ \\
\hline $765\left({ }^{95} \mathrm{Nb}\right)$ & $0.1839 \pm 0.0034(2 \%)$ & $0.1195 \pm 0.0023(2 \%)$ \\
\hline
\end{tabular}

The differences between Add Back mode and Singles mode in Table 17 are significant and should be taken into consideration. However, the usefulness of either of these modes should be carefully examined for each sample type being analyzed as other nuclides may interfere in different magnitudes.

\subsection{2 ${ }^{140} \mathrm{Ba}$ and ${ }^{140} \mathrm{La}$}

The ${ }^{140} \mathrm{Ba} /{ }^{140} \mathrm{La}$ fission product pair contains the "medium-lived" nuclides being analyzed in this work. The half-life of ${ }^{140} \mathrm{Ba}$ is 12.752 days and the half-life of ${ }^{140} \mathrm{La}$ is 1.6781 days, so the system will reach equilibrium about 11 days after irradiation. The analysis on ${ }^{140} \mathrm{Ba}$ gives insight into how the system responds to both singlet emissions and cascading emissions. The $163 \mathrm{keV}$ and $305 \mathrm{keV}$ photons are heavily paired together in the decay of ${ }^{140} \mathrm{Ba}$, whereas the $423 \mathrm{keV}$ and $537 \mathrm{keV}$ photons are mainly singlet emissions in the decay of ${ }^{140} \mathrm{Ba}$. In observing the spectra, the $423 \mathrm{keV}$ and $537 \mathrm{keV}$ peaks look to be free from interferences in both Add Back and Singles mode. The $163 \mathrm{keV}$ photon appears to be free from interference, but it has been observed that the $163 \mathrm{keV}$ emission from ${ }^{235} \mathrm{U}$ has an effect on the activity analysis of this peak, especially later in the sample's life. The $305 \mathrm{keV}$ photon is interfered with by ${ }^{105} \mathrm{Rh}$ at $306 \mathrm{keV}$, which has a 35.4 hour half-life. This interference appears in both the Singles and Add Back spectra. 
Looking at the singlet emissions of ${ }^{140} \mathrm{Ba}$, both the Add Back and Singles modes show good agreement with the beta counting estimations of the activity. In a few of the residual plots, it is shown that near the end of the counting times, the data points are not in agreement with the beta counting activity estimations. This may be because the peak to background ratio was low and the peak fitting results may not have been accurate. This would result in the activity calculation given by this peak also not being accurate.

Looking at the cascading emissions of ${ }^{140} \mathrm{Ba}$, both the Add Back and Singles modes still show decent agreement with the beta counting estimations of the activity except for the 305 keV peak in Add Back mode. By observing the activity residuals of each counting mode, it appears that the precision of Singles mode is significantly better than the precision shown in Add Back mode. This is because the expected amount of TCS is much less in Singles mode than in Add Back mode. TCS correction factors carry a great amount of uncertainty because they depend on several factors. They depend on the total efficiency of coinciding gamma-rays and Xrays, some of which fall outside the energy range for which the system was calibrated. They also depend on the decay schemes of the nuclides. If the decay scheme is large, then the number of possible coincident events can be quite large as well. Each transition, gamma-ray emission, and internal conversion coefficient has some associated uncertainty with it. Because the TCS factor includes so many factors, the associated uncertainty can be quite large. There may also be reason to believe that the total efficiency calculated from the peak-to-total ratios may be inaccurate due to high dead-time during some of the counts in the analysis. Because of these things, if there is a significant amount of cascading emissions associated with a photon being analyzed, Singles mode may be a better counting mode to use. Table 18 shows the expected peak counts per decay of ${ }^{140} \mathrm{Ba}$ in both Add Back and Singles modes. As expected, Singles mode will show more peak counts per decay for the cascading emissions of $163 \mathrm{keV}$ and $305 \mathrm{keV}$ while Add Back mode will show more peak counts per decay for the singlet emissions of $423 \mathrm{keV}$ and $537 \mathrm{keV}$. 
Table 18: Comparison of Add Back and Singles modes in detecting Ba-140

\begin{tabular}{|c|c|c|}
\hline Energy in keV (Nuclide) & $\begin{array}{c}\text { Add Back (Counts in Peak per } \\
\text { Decay) }\end{array}$ & $\begin{array}{c}\text { Singles (Counts in Peak per } \\
\text { Decay) }\end{array}$ \\
\hline $163\left({ }^{140} \mathrm{Ba}\right)$ & $0.0183 \pm 0.0023(13 \%)$ & $0.0254 \pm 0.0028(11 \%)$ \\
\hline $305\left({ }^{140} \mathrm{Ba}\right)$ & $0.00511 \pm 0.00104(20 \%)$ & $0.00989 \pm 0.00097(10 \%)$ \\
\hline $423\left({ }^{140} \mathrm{Ba}\right)$ & $0.00836 \pm 0.00088(11 \%)$ & $0.00616 \pm 0.00061(10 \%)$ \\
\hline $537\left({ }^{140} \mathrm{Ba}\right)$ & $0.0562 \pm 0.0066(12 \%)$ & $0.0393 \pm 0.0044(11 \%)$ \\
\hline
\end{tabular}

In Table 18, the percent error is shown in parentheses after the uncertainty. For the singlet emissions, the percent uncertainty is about the same. For the $305 \mathrm{keV}$ and $163 \mathrm{keV}$ gamma-rays however, the uncertainty is significantly different between the two modes. This is because of the amount of summing-out that occurs in each mode. The amount of summing-out can be calculated using:

$$
F_{S O, i j}=\frac{S_{i j}}{\varepsilon_{i j} \Gamma_{i j}}
$$

where:

$F_{S O, i j}$ is the fraction of summing-out that occurs for the transition between energy level $i$ and energy level $j$

$S_{i j}$ is the $i, j$ element of the $\mathbf{S}$ matrix in Equation (9.15)

$\varepsilon_{i j}$ is the full-energy peak efficiency of the transition between energy level $i$ and energy level $j$

$\Gamma_{i j}$ is the gamma abundance of the transition from energy level $i$ to energy level $j$ in gamma-rays per decay

Equation (10.12) was determined by taking the expected counts per decay in the presence of $\operatorname{TCS}\left(S_{i j}\right)$ and dividing by the expected counts per decay in the absence of TCS $\left(\varepsilon_{i j} \Gamma_{i j}\right)$. Using Equation (10.12), the summing out factor for each ${ }^{140}$ Ba gamma-ray emission in each counting mode is shown in Table 19. The fractions given in this Table represent the fraction of full-energy depositions that appear in the full-energy peak for that counting mode. The remaining fraction is summed-out of the full-energy peak into the sum peak or the continuum above the fullenergy peak. 
Table 19: TCS Factors for ${ }^{140} \mathrm{Ba}$ cascading gamma-rays

\begin{tabular}{|c|c|c|}
\hline Energy (keV) & $\begin{array}{c}\text { Add Back (Fraction of photons } \\
\text { unaffected by summing) }\end{array}$ & $\begin{array}{c}\text { Singles (Fraction of photons } \\
\text { unaffected by summing) }\end{array}$ \\
\hline 163 & 0.63 & 0.95 \\
\hline 305 & 0.36 & 0.87 \\
\hline
\end{tabular}

A major factor in the uncertainty of the $305 \mathrm{keV}$ activity calculation in Add Back mode is the uncertainty in the internal conversion coefficient of the $163 \mathrm{keV}$ photon. The main reason the $305 \mathrm{keV}$ photon retains so few of its full-energy depositions in Add Back mode is because it is heavily paired with the $163 \mathrm{keV}$ photon. In Singles mode, the activity calculations are not as influenced by the $163 \mathrm{keV}$ photon, so the uncertainty is less. In almost all the activity residuals plots from the 4130 sample, there appears to be a sharp downward trend, and then a leveling off shortly afterward. This is most likely due to the approximation of linear effects from the dead-time. In Chapter 8, it was stated that the fraction of events due to dead-time can be approximated linearly when the overall dead-time is low when compared with the live time. At the beginning of these counts, the dead-time was around $15 \%$, which can cause the approximation to break down. The dead-time effects can be drastically reduced by ensuring that wave forms are not recorded during counting. Had the waveform collection mode been turned off, the dead-time would have only been around $3 \%$ which would have caused no problems with the approximation.

The ${ }^{140}$ La gamma-rays are all part of cascading emissions, which is expected to heavily affect the Add Back spectrum. The $329 \mathrm{keV}$ and $1596 \mathrm{keV}$ peaks appear to be free from spectral interferences, and the $487 \mathrm{keV}$ and the $816 \mathrm{keV}$ peaks each have nearby peaks that slightly overlap. In observing the Singles activity residuals, it appears that all of the activity calculations based on each of the individual gamma-ray peaks are consistent with the beta emission activity estimations. The exception is the $1596 \mathrm{keV}$ photon in the 4130 sample near the end of the counts. This may be due to dead-time inaccuracies. When observing the Add Back activity residuals, all of the results are well above the activity estimations from beta counting. This is most likely due to the large amount of TCS that occurs with each of the emissions from ${ }^{140} \mathrm{La}$. The errors are also possibly due to inaccuracies in the peak-to-total ratios. Many of the samples taken to obtain the peak-to-total ratio produced very high dead-times (up to 60\%) which can 
severely influence the performance of the system. The peak-to-total ratios should be looked at again in order to produce better results for TCS corrections.

In almost all scenarios, analysis of samples for ${ }^{140}$ La should be performed using Singles mode due to the high amount of cascading emissions that occur in this nuclide. Table 20 shows the expected counts per decay from each of the main gamma-ray emissions in both Add Back and Singles modes.

Table 20: Comparison of Add Back and Singles modes in detecting La-140

\begin{tabular}{|c|c|c|}
\hline Energy in keV (Nuclide) & $\begin{array}{c}\text { Add Back (Counts in Peak per } \\
\text { Decay) }\end{array}$ & $\begin{array}{c}\text { Singles (Counts in Peak per } \\
\text { Decay) }\end{array}$ \\
\hline $329\left({ }^{140} \mathrm{La}\right)$ & $0.0118 \pm 0.0008(7 \%)$ & $0.0428 \pm 0.0025(6 \%)$ \\
\hline $487\left({ }^{140} \mathrm{La}\right)$ & $0.0363 \pm 0.0030(8 \%)$ & $0.0699 \pm 0.0036(5 \%)$ \\
\hline $816\left(^{140} \mathrm{La}\right)$ & $0.0290 \pm 0.0013(4 \%)$ & $0.0256 \pm 0.0008(3 \%)$ \\
\hline $1596\left({ }^{140} \mathrm{La}\right)$ & $0.0391 \pm 0.0026(7 \%)$ & $0.0564 \pm 0.0021(4 \%)$ \\
\hline
\end{tabular}

Of interest in Table 20 is the fact that all peaks are more prevalent in Singles mode except for the $816 \mathrm{keV}$ peak. Even though the $816 \mathrm{keV}$ photon is heavily paired with the $1596 \mathrm{keV}$ photon, the $329 \mathrm{keV}$ gamma-ray and the $487 \mathrm{keV}$ gamma-ray often sum-in to the $816 \mathrm{keV}$ peak, elevating the $816 \mathrm{keV}$ peak area. It should also be noted that the precision is better in Singles mode for all peaks of interest. Table 21 compares the effects of TCS for ${ }^{140}$ La in both counting modes.

Table 21: Effects of true coincidence summing in La-140 gamma-ray emissions

\begin{tabular}{|c|c|c|}
\hline Energy in keV (Nuclide) & $\begin{array}{c}\text { Add Back (Fraction of photons } \\
\text { unaffected by summing) }\end{array}$ & $\begin{array}{c}\text { Singles (Fraction of photons } \\
\text { unaffected by summing) }\end{array}$ \\
\hline $329\left({ }^{140} \mathrm{La}\right)$ & 0.18 & 0.83 \\
\hline $487\left({ }^{140} \mathrm{La}\right)$ & 0.32 & 0.87 \\
\hline $816\left({ }^{140} \mathrm{La}\right)$ & $0.50(0.36$ sum in $)$ & $0.92(0.04$ sum in) \\
\hline $1596\left({ }^{140} \mathrm{La}\right)$ & 0.36 & 0.89 \\
\hline
\end{tabular}

As evident by Table 20 and Table 21, ${ }^{140}$ La should be analyzed with Singles mode instead of Add Back mode almost without exception. The accuracy and the precision of Singles mode in analyzing ${ }^{140}$ La are significantly better than Add Back mode.

Gamma-gamma coincidence mode can be used when heavy interferences are observed around a peak of interest. If the peak of interest has a significant coinciding gamma-ray emission, then by gating on one of the two photons' energies, a spectrum free from 
interferences may be possible to attain. Figure 110 and Figure 111 show how an interference with the $305 \mathrm{keV}{ }^{140} \mathrm{Ba}$ gamma-ray emission can be eliminated by gating on the coinciding 163 keV gamma-ray emission from ${ }^{140} \mathrm{Ba}$.

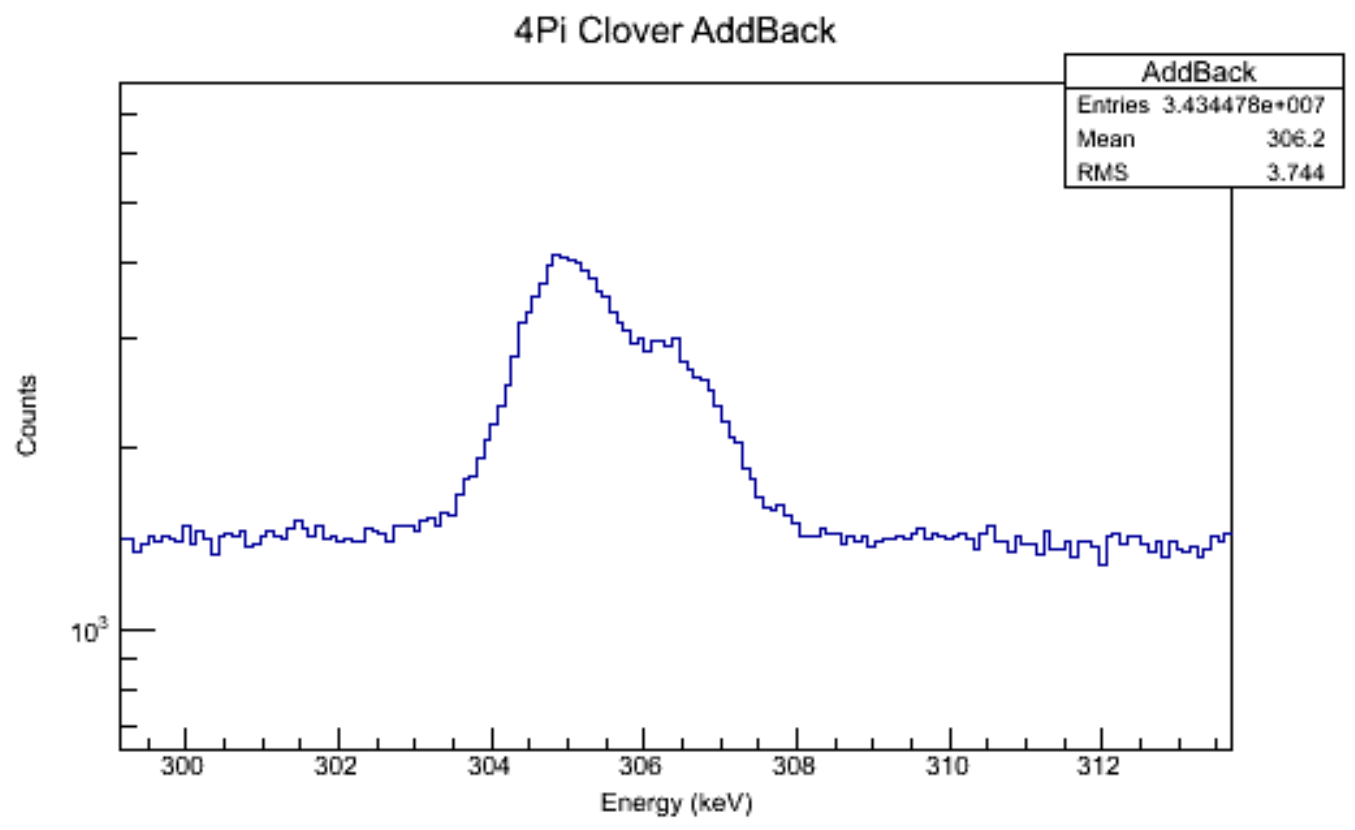

Figure 110: The $305 \mathrm{keV}$ peak is affected by an interfering peak around $306 \mathrm{keV}$

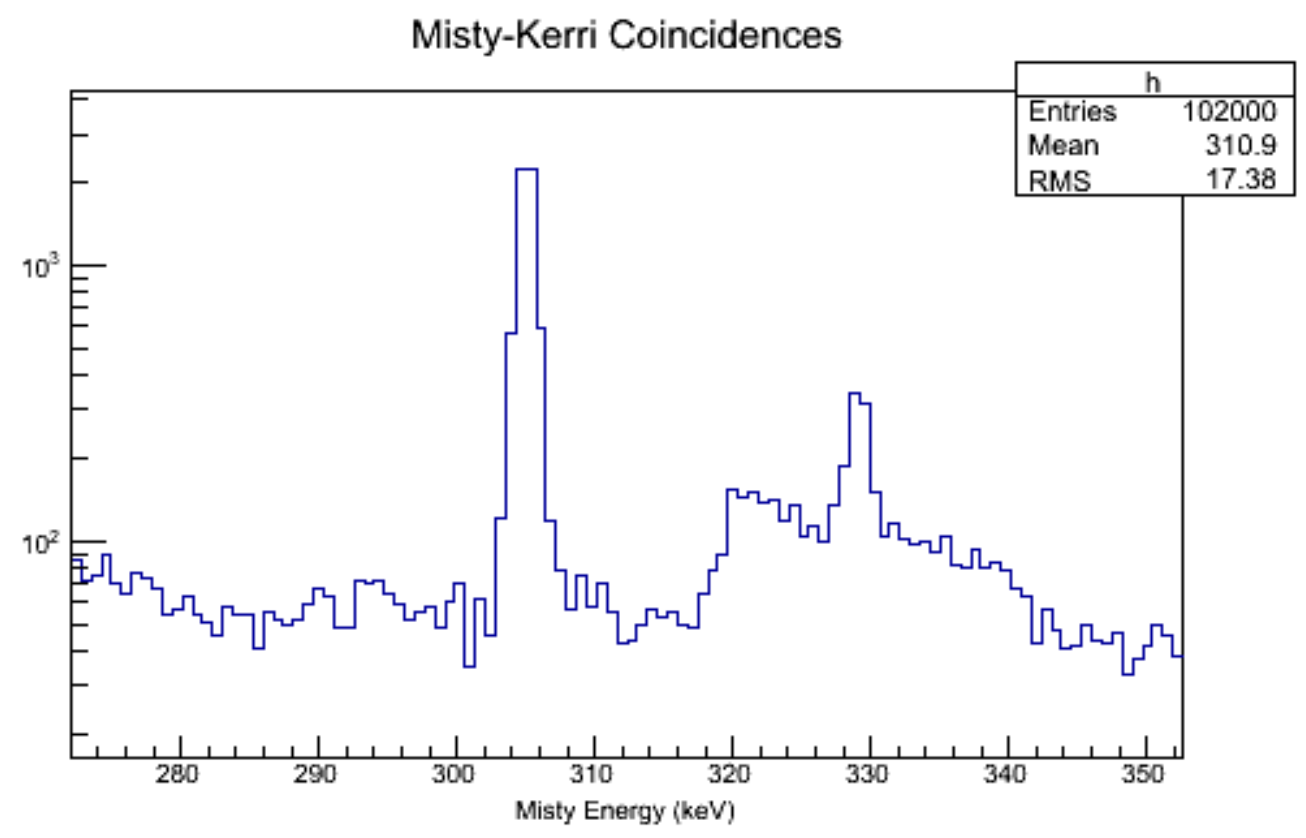

Figure 111: Interference from $306 \mathrm{keV}$ peak removed when gating on $163 \mathrm{keV}$ 
This can be especially useful when the interference is very close in energy to the peak of interest, such as that of the $163 \mathrm{keV}$ emissions from ${ }^{140} \mathrm{Ba}$ and ${ }^{235} \mathrm{U}$. If a plot is generated by gating on $305 \mathrm{keV}$, the $163 \mathrm{keV}$ peak will be free from contributions from ${ }^{235} \mathrm{U}$. Another advantage to using gamma-gamma coincidence mode is in the case of a decay scheme containing cascading gammas consisting of only two photons, such as ${ }^{140} \mathrm{Ba}$. In this case, there are no TCS effects to worry about, making the analysis much simpler.

Observing the ${ }^{140} \mathrm{Ba}$ gamma-gamma coincidence mode activity residuals for both samples, it appears that the data are consistent with the estimations given by beta counting. Once again, the residuals appear to have a downward slope before keeping a level value. The possible reasons for this have already been discussed above. The coincidences detected per decay of ${ }^{140} \mathrm{Ba}$ come out to be $0.00104 \pm 0.00017$ (17\%) when gating Kerri on $305 \mathrm{keV}$ and to $0.00102 \pm 0.00016(16 \%)$ when gating Misty on $305 \mathrm{keV}$. These numbers are vastly lower than for Add Back or Singles mode because the efficiency of the system when taking data this way is so much less. The high uncertainty in the internal conversion coefficient for the $163 \mathrm{keV}$ emission is a large factor in the lack of precision for this method.

The ${ }^{140}$ La gamma-gamma coincidence activity residuals show that the activity calculations for the coincidence between the $1596 \mathrm{keV}$ photon and the $329 \mathrm{keV}$ photon are above the estimated values obtained from beta counting. This is probably due to inaccurate peak-to-total ratios as described earlier. The activity calculations using the coincidence between the $1596 \mathrm{keV}$ photon and the $487 \mathrm{keV}$ photon from ${ }^{140} \mathrm{La}$ are accurate for some of the gating schemes but inaccurate for others. The activity residuals show the same pattern of a downward trend near the beginning of the counts and leveling off. In the gamma-gamma coincidence plots, it appears that the downward trend lasts longer than in the single photon plots. This is most likely due to the fact that the dead-time for the system is twice as high in gamma-gamma coincidence mode because the system is attempting to detect two photons. The system incurs approximately $25 \%$ dead-time in gamma-gamma coincidence mode during the first count, which is well about the $10 \%$ limit for the approximation on the dead-time corrections. Had the wave form recording been turned off, the dead-time would have been around 5\% for the earliest count. The activity calculations based on the coincidence between the $1596 \mathrm{keV}$ photon and the $816 \mathrm{keV}$ photon are lower than predicted for all gating schemes. The activity calculations based 
on the coincidence between the $487 \mathrm{keV}$ photon and the $329 \mathrm{keV}$ photon give mixed results in the 4126 sample, but are consistent with beta counting in the 4130 sample. Table 22 shows the expected coincidence counts per decay of ${ }^{140} \mathrm{La}$.

Table 22: Coincidences detected per decay of La-140

\begin{tabular}{|c|c|c|}
\hline $\begin{array}{c}\text { Energy of Coincident Photons } \\
\text { in keV (Nuclide) }\end{array}$ & $\begin{array}{c}\text { Kerri Gate (Coincidences } \\
\left.\text { Detected per Decay } \times 10^{-3}\right)\end{array}$ & $\begin{array}{c}\text { Misty Gate (Coincidences } \\
\left.\text { Detected per Decay } \times 10^{-3}\right)\end{array}$ \\
\hline $1596,329\left({ }^{140} \mathrm{La}\right)$ & $0.538 \pm 0.009$ & $0.550 \pm 0.010$ \\
\hline $1596,487\left({ }^{140} \mathrm{La}\right)$ & $1.63 \pm 0.03$ & $1.67 \pm 0.03$ \\
\hline $1596,816\left({ }^{140} \mathrm{La}\right)$ & $1.12 \pm 0.02$ & $1.12 \pm 0.02$ \\
\hline $487,329\left({ }^{140} \mathrm{La}\right)$ & $1.68 \pm 0.11$ & $1.67 \pm 0.11$ \\
\hline
\end{tabular}

\subsection{3 ${ }^{99}$ Mo and ${ }^{99 m} \mathrm{Tc}$}

The final fission product pair being analyzed in this work is ${ }^{99} \mathrm{Mo} /{ }^{99 \mathrm{~m}} \mathrm{Tc} .{ }^{99} \mathrm{Mo}$ has a halflife of 2.7476 days and ${ }^{99 \mathrm{~m}} \mathrm{Tc}$ has a half-life of 6.01 hours. These are the shortest lived nuclides being analyzed in this work. The emissions being analyzed for this fission product pair include both singlet and cascading gamma-rays. Something to note in this analysis is that the only analyzable gamma-ray from ${ }^{99 \mathrm{~m}} \mathrm{Tc}$ is the $141 \mathrm{keV}$ photon. This is also emitted by ${ }^{99}$ Mo when decaying directly to the ground state of ${ }^{99} \mathrm{Tc}$, so the $141 \mathrm{keV}$ peak observed in the spectrum will be populated by decays from both ${ }^{99} \mathrm{Mo}$ and ${ }^{99 \mathrm{~m}} \mathrm{Tc}$. By the time the 4126 sample started being counted (about 18 days after zero time), most of the original ${ }^{99}$ Mo content and the subsequent ${ }^{99 \mathrm{~m}} \mathrm{Tc}$ content had already decayed away. A few of the early spectra show visible peaks that can be attributed to ${ }^{99} \mathrm{Mo}$ and ${ }^{99 \mathrm{~m}} \mathrm{Tc}$, but the peaks are quite small. In the 4126 sample, the activity calculations based on the $778 \mathrm{keV}$ peak and most of the points in the $141 \mathrm{keV}$ peak are consistent with the beta counting estimations in both Add Back and Singles mode. Activity calculations from the $181 \mathrm{keV}$ peak give mixed results in both Add Back and Singles modes. Activity calculations using the $740 \mathrm{keV}$ peak appear to be high in Add Back mode and accurate in Singles mode when compared with the activity estimation from beta counting. Some of the data points near the end of the counts have large uncertainty bars. This is because the peaks being analyzed are very small and the counting statistics are poor. Looking at the 4130 sample, it appears as though the $778 \mathrm{keV}$ and $141 \mathrm{keV}$ activity calculations are in line with the beta counting estimations in both Add Back and Singles modes. The activity calculations from the $740 \mathrm{keV}$ peak are high in Add Back mode but appear to be accurate in Singles mode. The 181 keV photon activity calculations also give high results in Add Back mode and are mostly accurate 
in Singles mode; however in the early counts, the residuals lie just below the $-1 \sigma$ cutoff which may be due to inaccurate TCS factors.

In order to accurately predict the activity for ${ }^{99 \mathrm{~m}} \mathrm{Tc}$, the activity calculations using the $141 \mathrm{keV}$ emission need to be modified. The decay scheme used for the determination of the activity for ${ }^{99} \mathrm{Mo}$ assumed that ${ }^{99 \mathrm{~m}} \mathrm{Tc}$ is in transient equilibrium with ${ }^{99} \mathrm{Mo}$. For the purposes of this project, this is a good assumption because the first count did not occur until 110 hours after the end of bombardment. ${ }^{99 \mathrm{~m}} \mathrm{Tc}$ has a 6.0 hour half-life, and will reach equilibrium well before the first count starts. This happens approximately after seven daughter half-lives have passed, which would be around 42 hours after irradiation.

The change in daughter concentration can be obtained from the Bateman equations [54]:

$$
\frac{d N_{D}}{d t}=q \lambda_{P} N_{P}-\lambda_{D} N_{D}
$$

where:

$N_{D}$ is the daughter concentration

$N_{P}$ is the parent concentration

$q$ is the fraction of decays of the parent that go to the daughter

$\lambda_{P}$ is the decay constant of the parent

$\lambda_{D}$ is the decay constant of the daughter

It should be noted that for most parent-daughter pairs, $q$ is equal to 1.0 because the parent always decays to the daughter. However, for the ${ }^{99} \mathrm{Mo} /{ }^{99 \mathrm{~m}} \mathrm{Tc}$ pair, ${ }^{99} \mathrm{Mo}$ will only transition (directly or indirectly) to the metastable state of ${ }^{99} \mathrm{Tc}(87.6 \pm 1.9) \%$ of the time. Therefore, the first term in Equation (10.13) is multiplied by $q$. The activity of the daughter at time $t$ becomes:

$$
A_{D}(t)=\frac{q \lambda_{D} A_{P}(t)}{\lambda_{P}-\lambda_{D}}\left(e^{t\left(\lambda_{P}-\lambda_{D}\right)}-1\right)+A_{D 0} e^{-\lambda_{D} t}
$$

where:

$A_{D}(t)$ is the activity of the daughter at time $t$

$A_{P}(t)$ is the activity of the parent at time $t$

$A_{D O}$ is the activity of the daughter at $t=0$ 
Dividing both sides of Equation (10.14) by $A_{P}(t)$ :

$$
\begin{aligned}
& \frac{A_{D}(t)}{A_{P}(t)}=\frac{q \lambda_{D}}{\lambda_{P}-\lambda_{D}}\left(e^{t\left(\lambda_{P}-\lambda_{D}\right)}-1\right)+\frac{A_{D 0}}{A_{P}(t)} e^{-\lambda_{D} t} \\
& \frac{A_{D}(t)}{A_{P}(t)}=\frac{q \lambda_{D}}{\lambda_{P}-\lambda_{D}}\left(e^{t\left(\lambda_{P}-\lambda_{D}\right)}-1\right)+\frac{A_{D 0}}{A_{P 0}} e^{t\left(\lambda_{P}-\lambda_{D}\right)}
\end{aligned}
$$

where $A_{P O}$ is the activity of the parent at time $t=0$. Equilibrium will occur between the parent and the daughter if $\lambda_{D}$ is greater than $\lambda_{P}$. The ratio of parent activity to daughter activity in equilibrium is described by Equation (10.16) when $t \rightarrow \infty$ :

$$
\begin{aligned}
& \lim _{t \rightarrow \infty}\left(\frac{A_{D}(t)}{A_{P}(t)}\right)=\frac{-q \lambda_{D}}{\lambda_{P}-\lambda_{D}} \\
& \lim _{t \rightarrow \infty}\left(\frac{A_{D}(t)}{A_{P}(t)}\right)=\frac{q}{1-\frac{\lambda_{P}}{\lambda_{D}}}
\end{aligned}
$$

Plugging in the proper values, when the pair reaches equilibrium, the activity of ${ }^{99 \mathrm{~m}} \mathrm{Tc}$ will be 0.964 times the activity of ${ }^{99} \mathrm{Mo}$. Because the earliest count in either sample did not occur until 5 days after irradiation, the activity of ${ }^{99} \mathrm{Mo}$ was calculated assuming ${ }^{99 \mathrm{~m}} \mathrm{Tc}$ is in equilibrium with ${ }^{99} \mathrm{Mo}$ and using the $141 \mathrm{keV}$ photon; this activity was then multiplied by 0.964 to calculate the activity of ${ }^{99 \mathrm{~m}} \mathrm{Tc}$.

As with the other nuclides, the singlet emissions are better detected by Add Back mode and the cascading emissions are better detected by Singles mode. Table 23 shows the comparison for the analyzed photons between the two counting modes. As seen in the Table, the $141 \mathrm{keV}, 181 \mathrm{keV}$, and $740 \mathrm{keV}$ photons are expected to be better detected by Singles mode while the $778 \mathrm{keV}$ photon is expected to be better detected by Add Back mode. This is because the $778 \mathrm{keV}$ photon is a singlet emission while the other three photons have significant coincident emissions associated with them. 
Table 23: Comparison of Add Back and Singles modes in detecting Mo-99/Tc-99m

\begin{tabular}{|c|c|c|}
\hline Energy in keV (Nuclide) & $\begin{array}{c}\text { Add Back (Counts in Peak per } \\
\text { Decay) }\end{array}$ & $\begin{array}{c}\text { Singles (Counts in Peak per } \\
\text { Decay) }\end{array}$ \\
\hline $140\left({ }^{99 \mathrm{~m}} \mathrm{Tc} /{ }^{99} \mathrm{Mo}\right)$ & $0.396^{2} \pm 0.021(5 \%)$ & $0.412^{1} \pm 0.018(4 \%)$ \\
\hline $181\left({ }^{99} \mathrm{Mo}\right)$ & $0.0116 \pm 0.0008(7 \%)$ & $0.0244 \pm 0.0011(5 \%)$ \\
\hline $740\left({ }^{99} \mathrm{Mo}\right)$ & $0.00692 \pm 0.00056(8 \%)$ & $0.0134 \pm 0.0006(4 \%)$ \\
\hline $778\left({ }^{99} \mathrm{Mo}\right)$ & $0.00782 \pm 0.00036(5 \%)$ & $0.00506 \pm 0.00020(4 \%)$ \\
\hline
\end{tabular}

The fraction of full-energy depositions that are unaffected by summing out for each counting mode are presented in Table 24 . The $181 \mathrm{keV}$ and $740 \mathrm{keV}$ photons experience significant summing out effects in Add Back mode while all of the photons experience relatively low summing out effects in Singles mode.

Table 24: Effects of true coincidence summing in Mo-99/Tc-99m gamma-ray emissions

\begin{tabular}{|c|c|c|}
\hline Energy in keV (Nuclide) & $\begin{array}{c}\text { Add Back (Fraction of photons } \\
\text { unaffected by summing) }\end{array}$ & $\begin{array}{c}\text { Singles (Fraction of photons } \\
\text { unaffected by summing) }\end{array}$ \\
\hline $140\left({ }^{99 \mathrm{~m}} \mathrm{Tc} /{ }^{99} \mathrm{Mo}\right)$ & $0.96^{3}$ & $0.99^{2}$ \\
\hline $181\left({ }^{99} \mathrm{Mo}\right)$ & 0.41 & 0.91 \\
\hline $740\left({ }^{99} \mathrm{Mo}\right)$ & 0.30 & 0.90 \\
\hline
\end{tabular}

Observing the gamma-gamma coincidence mode plots, it appears that most of the data points show good agreement between the calculated activities and the beta counting activity estimations. There are a few random points that are off of the estimated values, but for the most part, they look good. These plots are also affected by high dead-times, so turning off the waveform capture is also expected to help these plots in terms of accuracy. Table 25 shows a comparison between the gating schemes for analyzing ${ }^{99} \mathrm{Mo}$. These coincidences are generally not affected by TCS.

Table 25: Coincidences detected per decay of Mo-99

\begin{tabular}{|c|c|c|}
\hline $\begin{array}{c}\text { Energy of Coincident Photons } \\
\text { in keV (Nuclide) }\end{array}$ & $\begin{array}{c}\text { Kerri Gate (Coincidences } \\
\left.\text { Detected per Decay } \times 10^{-3}\right)\end{array}$ & $\begin{array}{c}\text { Misty Gate (Coincidences } \\
\left.\text { Detected per Decay } \times 10^{-3}\right)\end{array}$ \\
\hline $740,140\left({ }^{99} \mathrm{Mo}\right)$ & $0.890 \pm 0.034$ & $0.870 \pm 0.034$ \\
\hline $740,181\left({ }^{99} \mathrm{Mo}\right)$ & $1.02 \pm 0.04$ & $0.988 \pm 0.038$ \\
\hline
\end{tabular}

\footnotetext{
${ }^{2}$ This is given in photons detected per decay of ${ }^{99} \mathrm{Mo}$ with ${ }^{99 \mathrm{~m}} \mathrm{Tc}$ in equilibrium

${ }^{3}$ This assumes ${ }^{99 \mathrm{~m}} \mathrm{Tc}$ is in transient equilibrium with ${ }^{99} \mathrm{Mo}$
} 


\subsubsection{Summary}

Throughout each of the parent-daughter pairs, there has been a trend that the photons that are emitted with significant coincidences are more accurate and precise in Singles mode than in Add Back mode. In fact, Add Back mode generally gives results that are not consistent with the activity estimations from beta counting, while the calculations from Singles mode are generally consistent for all photons. Add Back mode does give accurate results for singlet emissions. As mentioned previously, this implies that the calculation of the TCS factors may be inaccurate. A likely explanation is the high dead-times associated with many of the peak-tototal ratio counts, which can cause inaccuracies in the total efficiency calculated for each coincident photon. In all of the cases that Add Back mode produces inaccurate results, Singles mode produces better results and produces more full-energy detections per decay than Add Back mode. Even if Add Back mode gave accurate results, users would most likely want to use Singles mode for these photons because the peak areas will be higher and the detection limits will probably be lower. 


\section{Chapter 11: Age Dating Analysis}

One important property of nuclear forensics analysis is the "age" of the sample, which is interpreted as the time since neutron bombardment (detonation or irradiation in a reactor), the time since separation (reprocessing), or the time since enrichment [16]. For these samples, the proportion of the concentrations of the parents and daughters can be used to calculate the time since bombardment, assuming the daughter concentration at time $t=0$ is negligible [57]. Once the parents and daughters have reached equilibrium, the parent-daughter activity ratio is no longer usable for age dating. This is one of the main reasons researchers look at a variety of fission products to determine properties of the sample. Longer lived fission products can give age information over a longer period of time, but short lived fission products may give more precise age estimations as long as equilibrium has not been reached. To calculate the activity of the parent and daughter at each time step, the best known counting method for each photon will be used (based on the expected number of counts per decay). The proportion of the activities will then be calculated and the age of the sample can be estimated. Rearranging the Bateman equations [54], the equation that determines the age of the sample can be obtained from:

$$
\frac{N_{D}}{N_{P}}=\frac{\lambda_{P}}{\lambda_{P}-\lambda_{D}}\left(e^{t\left(\lambda_{P}-\lambda_{D}\right)}-1\right)
$$

which can be rearranged to give:

$$
t=\frac{\ln \left(\frac{\lambda_{P}-\lambda_{D}}{\lambda_{P}} \frac{N_{D}}{N_{P}}+1\right)}{\lambda_{P}-\lambda_{D}}
$$

where:

$N_{D} / N_{P}$ is the ratio of the number of daughter atoms in the sample to the number of parent atoms in the sample at time $t$

$\lambda_{P}$ is the decay constant of the parent nuclide

$\lambda_{D}$ is the decay constant of the daughter nuclide

$t$ is the age of the sample 
This assumes that there was no appreciable amount of the daughter nuclide in the sample at $t=0$. There may be appreciable daughter activity at time $t=0$ either from fission chain yield or parent decay during irradiation. If this is the case, Equation (11.1) should be rewritten as:

$$
\frac{N_{D}}{N_{P}}=\frac{\lambda_{P}}{\lambda_{P}-\lambda_{D}}\left(e^{t\left(\lambda_{P}-\lambda_{D}\right)}-1\right)+\frac{N_{D 0}}{N_{P 0}} e^{t\left(\lambda_{P}-\lambda_{D}\right)}
$$

where:

$N_{D O}$ is the initial number of daughter atoms

$N_{P O}$ is the initial number of parent atoms

This is rearranged so that Equation (11.2) becomes:

$$
t=\frac{\ln \left[\left(\frac{N_{D}}{N_{P}}+\frac{\lambda_{P}}{\lambda_{P}-\lambda_{D}}\right)\left(\frac{N_{D 0}}{N_{P 0}}+\frac{\lambda_{P}}{\lambda_{P}-\lambda_{D}}\right)^{-1}\right]}{\lambda_{P}-\lambda_{D}}
$$

The ratio of initial daughter atoms to initial parent atoms for Equation (11.3) and (11.4) can be estimated by fission chain yields and parent decay during irradiation. If these cannot be estimated well, then this method of age-dating becomes much less accurate.

Equilibrium will be reached in each parent-daughter pair at some time after the zero time as long as the half-life of the daughter is shorter than the half-life of the parent. The point at which equilibrium is observed depends on the amount of activity present from the parentdaughter pair, as well as the sensitivity of the detector system. Assuming the daughter nuclide concentration starts at 0.0 at zero time, the ratio of the concentrations of the daughter and the parent as a function of time is given by the equation above. If the half-life of the parent is longer than the half-life of the daughter, the exponent in Equation (11.1) is negative. If the daughter has a half-life longer than the parent, then no equilibrium will ever be reached. Assuming the parent half-life is longer than the daughter, as $t \rightarrow \infty$, the ratio in Equation (11.1) will be reduced to:

$$
\lim _{t \rightarrow \infty}\left(\frac{N_{D}}{N_{P}}\right)=\frac{\lambda_{P}}{\lambda_{D}-\lambda_{P}}
$$

The uncertainty in the peak areas of the parent and daughter [4] can be rearranged to give the uncertainty in the daughter-parent atom ratio: 


$$
\sigma_{R}=R \sqrt{\left(\frac{\sigma_{D}}{N_{D}}\right)^{2}+\left(\frac{\sigma_{P}}{N_{P}}\right)^{2}}
$$

where:

$R$ is the ratio of daughter atoms to parent atoms in the sample

$\sigma_{D}$ is the uncertainty in the number of daughter atoms

$\sigma_{P}$ is the uncertainty in the number of parent atoms

$N_{D}$ is the number of the daughter atoms in the sample

$N_{P}$ is the number of parent atoms in the sample

If the uncertainty in the daughter-to-parent ratio extends to the ratio expected at equilibrium, then the age dating results are no longer reliable. Once the equilibrium ratio has been reached, the age information using that parent-daughter pair has been lost.

\section{1 ${ }^{95} \mathrm{Zr}$ and ${ }^{95} \mathrm{Nb}$}

Both of these nuclides only produce singlet gamma-ray emissions and thus Add Back mode will be used to predict their activities. According to Equation (11.5), transient equilibrium occurs when the daughter-parent atom number ratio is 1.204. As an example of the age dating calculation, in the count taken on day 139 for the 4130 sample the following activities were calculated: the $724 \mathrm{keV}$ photon gave the ${ }^{95} \mathrm{Zr}$ activity to be $230 \pm 4 \mathrm{~Bq}$; the $756 \mathrm{keV}$ photon gave the ${ }^{95} \mathrm{Zr}$ activity to be $231 \pm 4 \mathrm{~Bq}$; and the $765 \mathrm{keV}$ photon gave the ${ }^{95} \mathrm{Nb}$ activity to be $83 \pm 2 \mathrm{~Bq}$. Averaging the ${ }^{95} \mathrm{Zr}$ activities gives an estimate of $231 \pm 3 \mathrm{~Bq}$ which corresponds to $(1.84 \pm 0.02) \mathrm{x}$ $10^{9}$ atoms. Because there is only one gamma-ray emission for ${ }^{95} \mathrm{Nb}$, the estimate of the activity is $83 \pm 2 \mathrm{~Bq}$ which corresponds to $(0.362 \pm 0.009) \times 10^{9}$ atoms. The proportion of the daughter to the parent concentration is $(0.362 \pm 0.009) \times 10^{9} /(1.84 \pm 0.02) \times 10^{9}=0.197 \pm 0.005$. Using Equation (11.2), it is shown that the age of the 4130 sample using the activities from the count taken on day 139 is estimated to be $19.9 \pm 0.5$ days old. In reality, the count was started exactly 19.824 days after zero time. The oldest count taken was from the 4126 sample. It was started 190 days after zero time. The daughter-parent ratio is still only $0.982 \pm 0.023$ which is still below the 1.204 limit. Observing Figure 38, the rollover from the daughter has occurred before this point, but equilibrium has not been reached in the pair until the slopes of the parent and 
daughter decay curves are equal. Throughout the counts, there is a consistent uncertainty of about $2.32 \%$ in the ratio, so it is predicted that when the ratio becomes $1.177 \pm 2.3 \%$, the age dating will be deemed inaccurate. This ratio is expected to occur around 421 days after zero time although the expected uncertainty at this time is 111 days. In most systems, equilibrium will be observed after six to seven daughter half-lives. This would correspond to between 210 and 245 days. However, because this system has a high sensitivity and low background contribution, equilibrium is not expected to be observed until about 12 daughter half-lives after irradiation. Figure 112 and Figure 113 show the estimates of the zero time of the sample at each count for the 4126 and 4130 sample using the ${ }^{95} \mathrm{Zr} /{ }^{95} \mathrm{Nb}$ fission product pair.

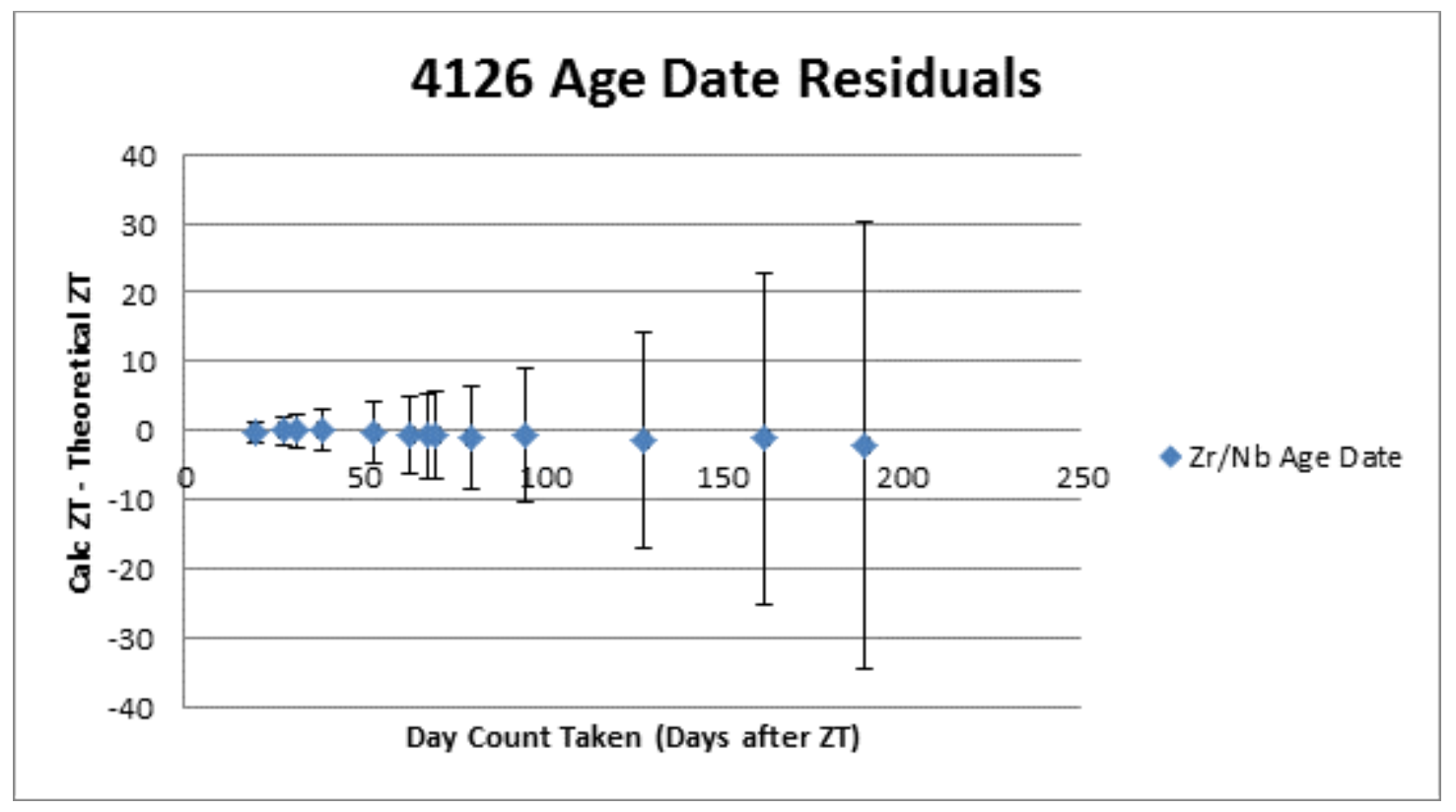

Figure 112: Using Zr-95 and Nb-95 to age date the 4126 sample 


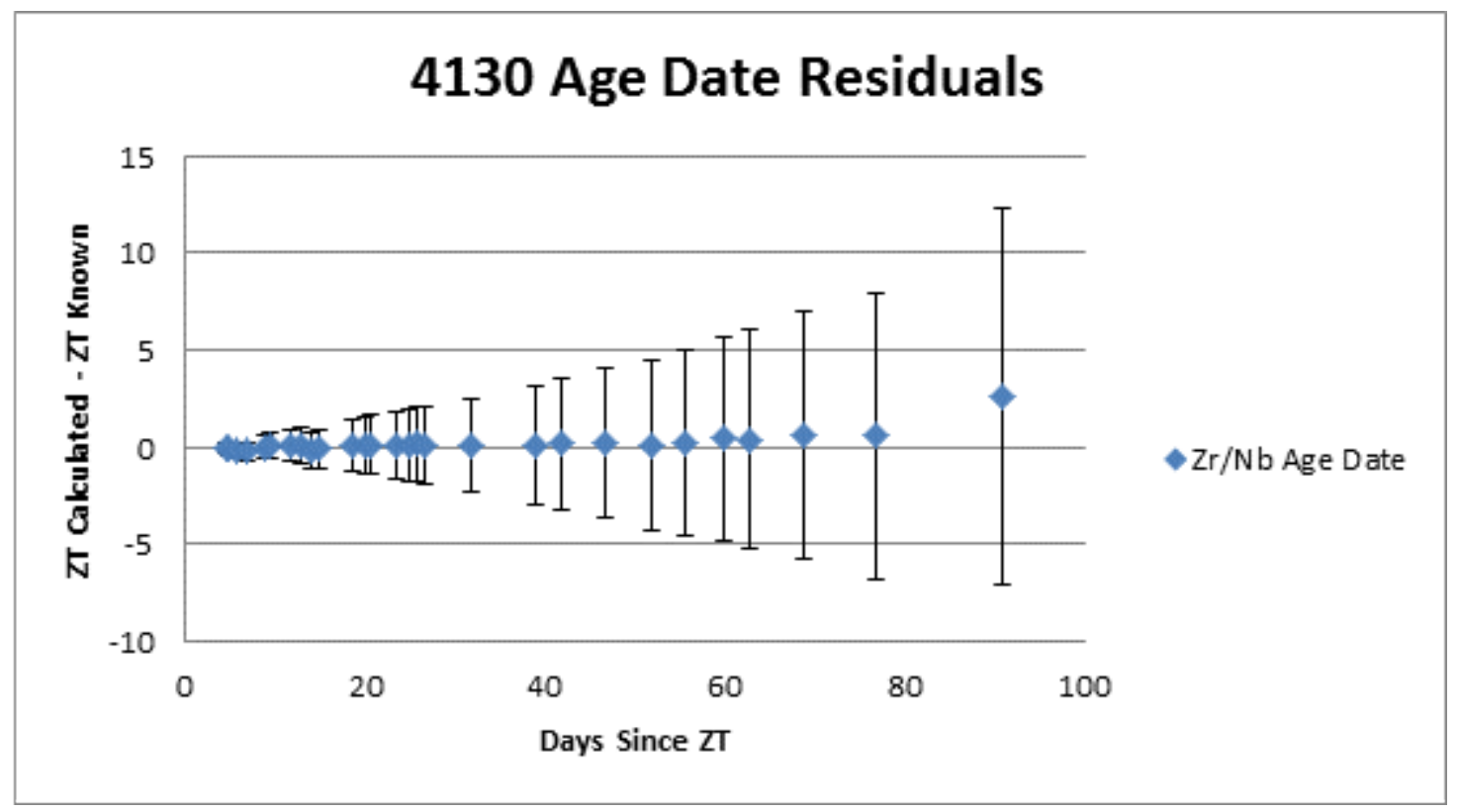

Figure 113: Using Zr-95 and Nb-95 to age date the 4130 sample

\section{$11.2{ }^{140} \mathrm{Ba}$ and ${ }^{140} \mathrm{La}$}

The main gamma-rays being observed from ${ }^{140} \mathrm{Ba}$ involve two singlet emissions and two cascading emissions. All of the observed gamma-rays from ${ }^{140} \mathrm{La}$ are cascading emissions. For the singlet emissions, Add Back mode will be used to estimate the activity, and for the cascading emissions, Singles mode will be used to estimate the activity. For this parent-daughter pair, the daughter-parent atom ratio will be 0.1515 at equilibrium. The first count from the 4126 sample, which was taken about 19.5 days after zero time, gave a daughter-parent atom ratio of $0.150 \pm$ 0.011. Because the uncertainty crosses the ratio at equilibrium, it is expected that the results will not be usable. The first count from the 4130 sample, which was started about 4.5 days after zero time, gave a daughter-parent ratio of $0.120 \pm 0.007$. It estimated the sample to be $4.38 \pm$ 0.63 days old. The final count that gave usable results occurred when the sample was 6.79 days old. It estimated the sample to be $7.1 \pm 1.9$ days old. The uncertainty in the daughter-parent ratio is consistently at around $5.9 \%$. From this, it is expected that the ${ }^{140} \mathrm{Ba} /{ }^{140} \mathrm{La}$ fission product pair can be used for age dating until the sample is 8.04 days old with an expected uncertainty of 2.8 days. Figure 114 shows the estimates of the zero time of the sample at each count for the 4130 sample using the ${ }^{140} \mathrm{Ba} /{ }^{140} \mathrm{La}$ fission product pair. 


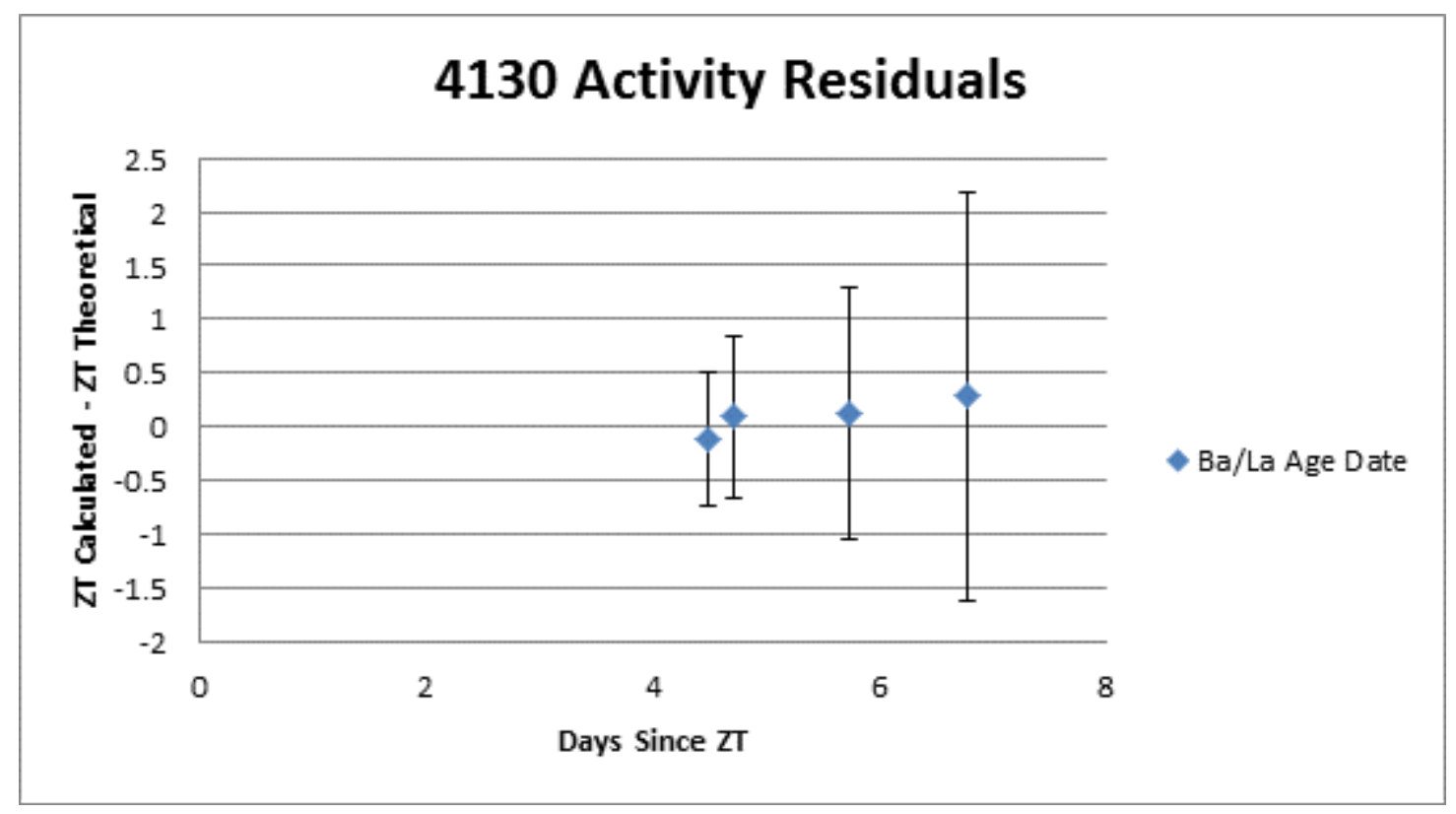

Figure 114: Using Ba-140 and La-140 to age date the 4130 sample

\section{3 ${ }^{99} \mathrm{Mo}$ and ${ }^{99 \mathrm{~m}} \mathrm{Tc}$}

Age dating using the ${ }^{99} \mathrm{Mo} /{ }^{99 \mathrm{~m}} \mathrm{Tc}$ pair is difficult because the $141 \mathrm{keV}$ photon is emitted both after transitioning to the metastable state of ${ }^{99} \mathrm{Tc}$ and without transitioning to the metastable state of ${ }^{99} \mathrm{Tc}$. From Section 10.3.3, it was shown that the activity of ${ }^{99 \mathrm{~m}} \mathrm{Tc}$ is $96.4 \%$ of the activity of ${ }^{99} \mathrm{Mo}$ in equilibrium. The decay scheme for ${ }^{99 \mathrm{~m}} \mathrm{Tc}$ found in Appendix 4 shows that the $141 \mathrm{keV}$ gamma-ray is emitted in $88.5 \%$ of all decays of ${ }^{99 \mathrm{~m}} \mathrm{Tc}$. The decay scheme for ${ }^{99} \mathrm{Mo}$ in Appendix 4 shows that the $141 \mathrm{keV}$ gamma-ray is emitted in $89.6 \%$ of all decays of ${ }^{99} \mathrm{Mo}$. This decay scheme assumes that ${ }^{99 \mathrm{~m}} \mathrm{Tc}$ is in equilibrium with ${ }^{99} \mathrm{Mo}$. Therefore, the fraction of decays that the $141 \mathrm{keV}$ gamma-ray is emitted from ${ }^{99 \mathrm{~m}} \mathrm{Tc}$ is given by:

$$
F_{T c-99 m}=\frac{\frac{A_{T c-99 m}}{A_{M o-99}} * I_{T c-99 m}(141 \mathrm{keV})}{I_{M o-99}(141 \mathrm{keV})}
$$

where:

$F_{T c-99 m}$ is the fraction of $141 \mathrm{keV}$ photons that are emitted after ${ }^{99}$ Mo transitions to the metastable state of ${ }^{99} \mathrm{Tc}$

$A_{T c-99 m} / A_{\text {Mo-9g }}$ is the activity ratio between ${ }^{99 \mathrm{~m}} \mathrm{Tc}$ and ${ }^{99} \mathrm{Mo}$ at equilibrium

$I_{T c-99 m}(141 \mathrm{keV})$ is the intensity of the $141 \mathrm{keV}$ gamma-ray from ${ }^{99 \mathrm{~m}} \mathrm{Tc}$

$I_{\text {Mo-99 }}(141 \mathrm{keV})$ is the intensity of the $141 \mathrm{keV}$ gamma-ray from ${ }^{99} \mathrm{Mo}$ 
Equation (11.7) predicts that $95.2 \%$ of the time the $141 \mathrm{keV}$ photon will originate from the metastable level of ${ }^{99} \mathrm{Tc}$. At creation, the $141 \mathrm{keV}$ photon will originate from the metastable level of ${ }^{99} \mathrm{Tc} 0 \%$ of the time. Before equilibrium is reached, this parent-daughter pair can be used for age dating; however, the TCS factors would have to be known to calculate the contribution of the $141 \mathrm{keV}$ photons that do not originate from ${ }^{99 \mathrm{~m}} \mathrm{Tc}$. As it stands, the TCS factors have only been calculated for ${ }^{99} \mathrm{Mo}$ when ${ }^{99 \mathrm{~m}} \mathrm{Tc}$ is in equilibrium. With the half-life of ${ }^{99 \mathrm{~m}} \mathrm{Tc}$ being so short, equilibrium is expected to be observed within 42-60 hours, and age dating of samples at this level will probably not be necessary. If required though, it may be possible to calculate the age if the TCS factors could be computed for the $141 \mathrm{keV}$ photon at various points before transient equilibrium occurs. Buildup of ${ }^{99 \mathrm{~m}} \mathrm{Tc}$ during irradiation may also add a significant amount of uncertainty to this whole process. 


\section{Chapter 12: Detection Limits}

When analyzing spectra, the presence of background and interfering peaks can have profound effects on the analysis and determination of significant nuclides. Many times, the activity of a nuclide is so low that the peaks it produces cannot be distinguished from the background spectrum or nearby peaks from interfering radionuclides. The detection limit determines the lowest expected activity level that a system can observe and indicate that a signal is present within a given risk tolerance [58].

\subsection{Theory}

There are three levels of detection that can be distinguished [58]. The first is the Critical Level $\left(L_{C}\right)$ which is the net signal level above which a signal can be termed to be detected. The Critical Level is used when looking at a spectrum to determine if a peak-like anomaly in the spectrum should be considered an actual peak or just a statistical fluctuation of the background. The Detection Limit $\left(L_{D}\right)$ is the signal level above which we can expect to detect a signal, and is determined a priori. If a nuclide in a sample has an activity above the Detection Limit, then a given spectrum containing the nuclide has a significant probability of producing a peak above the Critical Limit, and thus will be considered an actual peak as opposed to a statistical fluctuation of the background. The Quantitative Limit $\left(L_{Q}\right)$ is the level at which the measurement is precise enough to quantitatively determine the level of the signal and is generally well-above the Detection Limit. This information and the following derivation are taken from Currie's classic publication [58].

There are two types of error when determining signals. A Type I error occurs when a signal is said to be present when in fact no signal is there - a false positive. A Type II error occurs when a signal is said to be absent, when in fact it is there - a false negative. The Critical Level is the signal that must be present in a spectrum in order to have a significant probability of avoiding a Type I error. It is given by the following equation: 


$$
L_{C}=k_{\alpha} \sigma_{0}
$$

where:

$k_{\alpha}$ is a coefficient corresponding to the level of significance required by the observer in order to avoid a Type I error

$\sigma_{0}$ is the standard deviation of the signal given by a blank standard (i.e. a measure of the statistical fluctuation of the background)

The term "significance" is a statistical term that can mean different things depending on the level of reliability that the researcher wishes to produce. In many statistical evaluations, a significant result is achieved when a researcher can be at least $95 \%$ confident that the result is not due to statistical fluctuations [59].

The Detection Limit is a signal that is calculated from the Critical Level. It corresponds to the level of signal that must be present, such that a signal below the Critical Level will result in a Type II error. It is given by:

$$
L_{D}=L_{C}+k_{\beta} \sigma_{D}
$$

where:

$k_{B}$ is a coefficient corresponding to the level of significance required by the observer to avoid a Type II error $\sigma_{D}$ is the standard deviation of the signal if the signal were equal to the detection limit

The Quantitative Limit is the level of signal that must be present in order to put a quantitative value to the signal and is not related to the Critical Level or the Detection Limit, but is rather related to the sensitivity of the system being used to analyze the samples.

Detection limits can be determined for various nuclides from background or "blank" spectra, assuming there are no interferences for the peak in the spectrum being analyzed. However, this is often not the case with forensics samples, especially those containing fission products. The Compton continuum from high energy photons will contribute to the baseline for lower-energy peaks, and thus detection limits will be higher than they would be if the baseline at that location were lower. Because of these factors, detection limits can only be specified for samples that are similar in nature to the sample being analyzed for detection limits. For 
example, one cannot obtain a detection limit of a nuclide in a blank sample and declare it to be the detection limit for the same nuclide in a spent fuel sample. Therefore, detection limits will be determined for the irradiated uranium samples at various ages, as well as for a blank sample. Detection limits also depend on the count time, and should be taken into account when analyzing low-level samples [58].

When analyzing various peaks of the same nuclide for detection limits, the energy of each of the peaks and the gamma-ray abundance of the analyzed peak must be taken into consideration. The number of counts necessary to be distinguished from the background at a certain location on the spectrum will be dependent on the continuum and interferences present. Because the efficiency of the system is energy-dependent and the gamma abundances for each nuclide can vary, the detection limit in terms of activity for a specific nuclide may be different for the various photons emitted. The net signal can be determined by the following equation [58]:

$$
S=G-B-I
$$

where:

$S$ is the net signal

$\mathrm{G}$ is the gross signal in the spectrum

$B$ is the signal from the "blank" spectrum

$\mathrm{I}$ is the signal from any interferences in the system

The variance of the net signal will be:

$$
\sigma_{S}^{2}=\sigma_{G}^{2}+\sigma_{B}^{2}+\sigma_{I}^{2}
$$

where:

$\sigma_{\mathrm{S}}{ }^{2}$ is the variance of the net signal

$\sigma_{G}{ }^{2}$ is the variance of the gross signal

$\sigma_{B}{ }^{2}$ is the variance of the background signal

$\sigma_{1}^{2}$ is the variance of the interfering signal 
Assuming the signal is Poisson distributed, the mean of the gross counts becomes [4]:

$$
\begin{gathered}
\mu_{G}=\sigma_{G}^{2} \\
\sigma_{S}^{2}=\mu_{G}+\sigma_{B}^{2}+\sigma_{I}^{2}
\end{gathered}
$$

where $\mu_{G}$ is the true area of the gross signal. The gross signal is the sum of the desired signal, background signal, and interference signal, therefore Equation (12.6) becomes:

$$
\sigma_{S}^{2}=\mu_{S}+\mu_{B}+\mu_{I}+\sigma_{B}^{2}+\sigma_{I}^{2}
$$

where:

$\mu_{s}$ represents the true mean value of the signal from the sample

$\mu_{B}$ represents the true mean value of the signal from the background

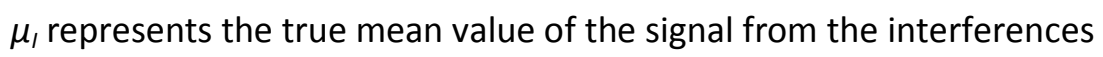

The true values of these signals are unknown, but the measured values are assumed to be a good indication of the true mean values [4]. Equation (12.7) then becomes:

$$
\sigma_{S}^{2}=S+B+I+\sigma_{B}^{2}+\sigma_{I}^{2}
$$

When the true net signal is $0\left(\mu_{s}=0\right)$, then the variance of a signal form a blank standard becomes:

$$
\sigma_{0}^{2}=\mu_{B}+\mu_{I}+\sigma_{B}^{2}+\sigma_{I}^{2}
$$

When the true net signal is equal to the detection limit $L_{D}$, the variance of the signal becomes:

$$
\sigma_{D}^{2}=L_{D}+\mu_{B}+\mu_{I}+\sigma_{B}^{2}+\sigma_{I}^{2}
$$

Substituting Equation (12.9) into Equation (12.1), the equation for the Critical Level becomes:

$$
L_{C}=k_{\alpha} \sqrt{\mu_{B}+\mu_{I}+\sigma_{B}^{2}+\sigma_{I}^{2}}
$$

Substituting Equation (12.10) into Equation (12.2), the equation for the Detection Limit becomes:

$$
L_{D}=L_{C}+k_{\beta} \sqrt{L_{D}+\mu_{B}+\mu_{I}+\sigma_{B}^{2}+\sigma_{I}^{2}}
$$

In Equation (12.12), $\mathrm{L}_{\mathrm{D}}$ is on both sides. Assuming $k_{\alpha}=k_{B}=k$, by substituting Equation (12.11) into Equation (12.12) and performing the necessary algebra, the equation for the Detection Limit becomes:

$$
L_{D}=k^{2}+2 L_{C}
$$


The above analysis is primarily intended for use with Single Channel Analyzers (SCA's) or plain counters in which a number is produced from each measurement [59]. Gamma-ray spectroscopy utilizes Multi-Channel Analyzers and thus produces counts in each bin that corresponds to counts from certain energy ranges. The terms in the Critical Limits and Detection Limits should be further defined for use with gamma-spectroscopy.

Using the above equations in gamma-ray spectroscopy, an estimation needs to be determined for the "zero" signal which includes the continuum and any interfering peaks in the spectrum. To truly measure the "zero" signal in a particular sample, the nuclide of interest needs to be extracted from a sample, and the spectrum should be analyzed at the peak locations of the nuclide being analyzed. This is ideal but impractical. Various methods have been introduced to estimate the zero-signal for a particular peak, and the method used in this research comes from a publication by Lars-Erik De Geer [59]. This method is presented here for reference.

If the background is not well-known and no interferences are present, the equation for the Critical Limit becomes:

$$
L_{C}=k \sqrt{\mu_{B}\left(1+\frac{1}{m}\right)}
$$

where:

$k$ and $\mu_{B}$ are defined above

$m$ is the number of times the background has been measured

For most analyses, the number of counts is appreciable and the level of significance requires $k<$ 5 so that the Detection Limit can be approximated by:

$$
L_{D} \cong 2 L_{C}
$$

This is equivalent to disregarding the small increase in the variance of the true signal's gross count when the true signal goes from zero to the Detection Limit. The first problem encountered in applying the SCA principles to gamma spectroscopy is how much of the observed peak to incorporate into the Detection Limit calculations. Only taking the peak centroid will not use much of the data available from the peak, but taking a wide part of the spectrum will incorporate too much of the background in the procedure. There is some middle 
ground that will give an optimal Limit of Detection. De Geer solves this problem by assuming the SCA is gated over a certain energy range that incorporates a specified part of the peak [59]. The peak is assumed to be Gaussian, and the energy range gated for the SCA is $\pm n \sigma$ or $2 n \sigma$. The area of the peak incorporated in the SCA is then defined as:

$$
S=A_{0} \operatorname{erf}\left(\frac{n}{\sqrt{2}}\right)
$$

where:

$A_{0}$ is the area of the full Gaussian peak

$n$ is the number of standard deviations used for the energy gate for the SCA

The background under the peak is $2 n \sigma B_{E}$ where $B_{E}$ is the average number of background events per channel in the area of the spectrum being analyzed. If the variance of the gross signal is Poisson, then it is equal to $S+2 n \sigma B_{E}$. Assuming the background is well-known (i.e. $m \rightarrow \infty)$, the variance of the net signal $S$ in the midst of the background will also be $S+2 n \sigma B_{E}$ because the number of counts in a given segment of the continuum follows a Poisson distribution [59]. In order to minimize the relative uncertainty of the net signal, the quantity to minimize is:

$$
y=\frac{\sqrt{S+2 n \sigma B_{E}}}{S}
$$

where $y$ is the relative uncertainty expected in the net signal. In observing this value in signals near the detection limit, De Geer shows that the optimal width of the peak to gate on the SCA is 1.25 times the FWHM [59]. This will minimize the variance associated with the net signal in the midst of a well-known background. This width incorporates about $85.9 \%$ of the peak, so when Detection Limits are calculated using this width, the result must be divided by 0.859 to determine the true Detection Limit for the peak being analyzed. Using this method, the background signal needed for the Critical Limit equation will be defined as:

$$
\mu_{B}=1.25 B_{E} \omega_{E}
$$

where $\omega_{E}$ is defined as the FWHM expressed in channels. Equation (12.14) for gamma spectra then becomes: 


$$
L_{C}=k \sqrt{1.25 B_{E} \omega_{E}\left(1+\frac{1}{m}\right)}
$$

De Geer then argues that although $m$ is defined as the number of background measurements made in the SCA, the background in a gamma spectrum can be observed over many "SCA widths" [59]. In fact, $m$ can be considered infinite "if the[background] line just varies in a reasonably smooth way" [59]. Interfering peaks should be handled independently. Taking $m$ to be large, Equation (12.19) becomes:

$$
L_{C}=k \sqrt{1.25 B_{E} \omega_{E}}
$$

Substituting Equation (12.15) into Equation (12.20), the Detection Limit can be approximated by:

$$
L_{D} \cong 2 k \sqrt{1.25 B_{E} \omega_{E}}
$$

In these equations, several assumptions have been made [59]. First, the peaks are assumed to be Gaussian in nature. In actuality, the peaks are slightly asymmetric, but the approximation will hold for the purposes of this research. Another assumption is that the user is willing to accept risks of Type I or Type II errors at levels above 0.0005 and that backgrounds counts are reasonably high (above 20 counts in the selected region). If these assumption do not hold true, then the Gaussian distribution of the background counts (including the mean = variance relation) will not hold and thus Poisson statistics will need to be used instead. This is not the case for this research and thus will not be explored further.

It should be noted that the detection limits will be different for different count times. Let the background count rate be given by $C R_{B}$ and let the peak count rate be given by $C R_{p}$. Also let the count time be represented by $T$. Equation (12.21) then becomes:

$$
0.859 * C R_{P} * T \cong 2 k \sqrt{1.25 C R_{B} T \omega_{E}}
$$

where 0.859 was introduced on the left hand side because this is the percentage of the peak represented by the energy range $1.25 \omega_{E}$. Equation (12.22) can be rearranged to give:

$$
C R_{P} \cong \frac{2 k}{0.859} \sqrt{\frac{1.25 C R_{B} \omega_{E}}{T}}
$$

$C R_{P}$ is the peak count rate necessary to obtain $L_{D}$ counts in a time $T$ in the energy range corresponding to $1.25 \omega_{E}$. Equation (12.23) shows that this count rate is dependent on the count time $T$. 
For this research detection limits will be calculated for each peak in four different types of samples. The first will be detection limits of samples with activities near background levels. This will be the absolute detection limit which indicates the lowest possible activity detectable in any sample. The second will be the detection limits in a relatively new sample around 4 days after irradiation. The third sample type will be a sample that is one month old. The final sample type will be a sample that is five months old. The detection limits found in these samples should be comparable to the detection limits of any sample likely to be encountered with the exception of a sample less than one day after zero time as there are many more short-lived nuclides present in these samples that will be gone after four days. For each spectrum, the detection limits have been extrapolated for various live times based on the count rates observed.

\subsection{Detection Limits in Near Background Spectrum}

The background spectrum taken for this part of the research was taken for 480,000 seconds. The dead-time in this spectrum is expected to be quite low because the count rate is only 15.2 counts per second across the whole spectrum (3000 keV). This background spectrum was taken in Add Back mode. In reality, the Add Back spectrum and Singles spectrum should be very similar because many of the counts recorded are either singlets or are counts produced from pair production. The detection limits for Singles mode should be investigated further as a Singles mode spectrum was not available for this background count.

The detection limits of each nuclide based on their main photons are displayed in Table 26 for a near background sample in Add Back mode for a variety of counting times. Table 27 shows the detection limits for each peak when the sample is taken in Singles mode. The background is assumed to be the same for both Add Back and Singles mode, though the validity of this assumption should be investigated further. For each of the values, the nuclide's activity has been corrected for decay during counting and is reflective of the activity of the nuclide at the beginning of the count. If the half-life is long compared to the count time, then the activity at the end of the count will be about the same as the activity at the start of the count. If the half-life is short compared to the count time, then the activity at the end of the count will be significantly lower than the activity at the start of the count. For this reason, the activity necessary to produce $L_{D}$ counts in the energy range in a time $T$ is corrected for counting time by 
Equation (9.23). Each of the daughter nuclides are assumed to be in equilibrium with their parents for the purposes of correcting for decay during counting.

Table 26: Detection limits in Bq for a near background sample in Add Back mode.

A bolded value indicates that analyzing the specific photon in Add Back mode is estimated to provide the best counting statistics for a particular nuclide.

\begin{tabular}{|c|c|c|c|c|c|}
\hline \multirow{2}{*}{ Nuclide } & $\begin{array}{c}\text { Peak Energy } \\
(\mathrm{keV})\end{array}$ & \multicolumn{4}{|c|}{ Counting Live Time } \\
\cline { 2 - 6 } & 140 & $60 \mathrm{~s}$ & $60 \mathrm{~min}$ & $24 \mathrm{~h}$ & $7 \mathrm{~d}$ \\
\hline${ }^{99 \mathrm{~m}} \mathrm{Tc} /{ }^{99} \mathrm{Mo}$ & 0.37 & 0.032 & 0.0070 & 0.0050 \\
\hline \multirow{3}{*}{$99 \mathrm{Mo}$} & 181 & 12.59 & 1.120 & 0.2435 & 0.1717 \\
\cline { 2 - 6 } & 740 & 15.71 & 1.182 & 0.2482 & 0.1740 \\
\cline { 2 - 6 } & 778 & 13.64 & 1.011 & 0.2117 & 0.1483 \\
\hline \multirow{3}{*}{${ }^{140} \mathrm{Ba}$} & 163 & 7.92 & 0.701 & 0.1529 & 0.1078 \\
\cline { 2 - 6 } & 305 & 27.35 & 2.378 & 0.5171 & 0.3644 \\
\cline { 2 - 6 } & 423 & 14.99 & 1.230 & 0.2646 & 0.1861 \\
\hline \multirow{3}{*}{${ }^{140} \mathrm{La}$} & $\mathbf{5 3 7}$ & $\mathbf{2 . 0 7}$ & $\mathbf{0 . 1 6 2}$ & $\mathbf{0 . 0 3 4 6}$ & $\mathbf{0 . 0 2 4 3}$ \\
\cline { 2 - 6 } & 329 & 11.62 & 1.001 & 0.2172 & 0.1530 \\
\cline { 2 - 6 } & 487 & 3.41 & 0.278 & 0.0596 & 0.0419 \\
\cline { 2 - 6 } & 1596 & 3.68 & 0.271 & 0.0570 & 0.0400 \\
\hline \multirow{2}{*}{${ }^{95} \mathrm{Zr}$} & 724 & 2.30 & 0.146 & 0.0296 & 0.0206 \\
\hline${ }^{95} \mathrm{Nb}$ & $\mathbf{7 5 6}$ & $\mathbf{1 . 0 6}$ & $\mathbf{0 . 0 7 9}$ & $\mathbf{0 . 0 1 4 8}$ & 0.0070 \\
\hline & $\mathbf{7 6 5}$ & $\mathbf{0 . 5 8}$ & $\mathbf{0 . 0 4 3}$ & $\mathbf{0 . 0 0 8 1}$ & $\mathbf{0 . 0 0 5 3}$ \\
\hline
\end{tabular}


Table 27: Detection limits in Bq for a near background sample in Singles mode.

A bolded value indicates that analyzing the specific photon in Singles mode is estimated to provide the best counting statistics for a particular nuclide.

\begin{tabular}{|c|c|c|c|c|c|}
\hline \multirow{2}{*}{ Nuclide } & $\begin{array}{c}\text { Peak Energy } \\
(\mathrm{keV})\end{array}$ & \multicolumn{4}{|c|}{ Counting Live Time } \\
\cline { 2 - 6 } & $\mathbf{1 4 0}$ & $\mathbf{0 . 3 5}$ & $\mathbf{0 . 0 3 1}$ & $\mathbf{0 . 0 0 6 8}$ & $\mathbf{0 . 0 0 4 8}$ \\
\hline${ }^{99 \mathrm{~m}} \mathrm{Tc} /{ }^{99} \mathrm{Mo}$ & $\mathbf{1 8 1}$ & $\mathbf{6 . 4 2}$ & $\mathbf{0 . 5 7 1}$ & $\mathbf{0 . 1 2 4 2}$ & $\mathbf{0 . 0 8 7 5}$ \\
\hline \multirow{3}{*}{${ }^{99} \mathrm{Mo}$} & 740 & 8.09 & 0.608 & 0.1278 & 0.0896 \\
\cline { 2 - 6 } & 778 & 21.08 & 1.563 & 0.3272 & 0.2293 \\
\cline { 2 - 6 } & 163 & 5.70 & 0.505 & 0.0999 & 0.0438 \\
\hline \multirow{3}{*}{${ }^{140} \mathrm{Ba}$} & 305 & 14.13 & 1.229 & 0.2426 & 0.1063 \\
\cline { 2 - 6 } & 423 & 20.34 & 1.670 & 0.3260 & 0.1425 \\
\cline { 2 - 6 } & 537 & 2.96 & 0.232 & 0.0448 & 0.0196 \\
\hline \multirow{3}{*}{${ }^{140} \mathrm{La}$} & 329 & 3.20 & 0.276 & 0.0543 & 0.0238 \\
\cline { 2 - 6 } & 487 & 1.77 & 0.144 & 0.0281 & 0.0123 \\
\cline { 2 - 6 } & 816 & 4.16 & 0.307 & 0.0586 & 0.0255 \\
\cline { 2 - 6 } & $\mathbf{1 5 9 6}$ & $\mathbf{1 . 5 9}$ & $\mathbf{0 . 1 0 1}$ & $\mathbf{0 . 0 1 8 6}$ & $\mathbf{0 . 0 0 8 1}$ \\
\hline \multirow{2}{*}{${ }^{95} \mathrm{Zr}$} & 724 & 1.96 & 0.147 & 0.0276 & 0.0106 \\
\hline \multirow{2}{*}{${ }^{95} \mathrm{Nb}$} & 756 & 1.63 & 0.121 & 0.0227 & 0.0087 \\
\hline
\end{tabular}

\subsection{Detection Limits for Six Month Old Sample}

The oldest spectrum available is the final spectrum taken with the 4126 sample. This spectrum was taken 190.0 days after zero time. Because of the long-lived nuclides present in this sample, the detection limits are expected to be higher than that of the background sample. Table 28 and Table 29 show the detection limits for these samples in Add Back and Singles modes respectively. It is worth noting that the area around $141 \mathrm{keV}$ is populated by interfering peaks, which may originate from both ${ }^{77 \mathrm{~m}} \mathrm{Ge}$ and ${ }^{116 \mathrm{~m}} \mathrm{In}$. ${ }^{77 \mathrm{~m}} \mathrm{Ge}$ is created by the cosmic neutron flux activating the detector material via an $(n, \gamma)$ reaction. Part of the material used to bind the detector crystal to the cryostat uses indium. This indium can be activated from the cosmic neutron flux to create ${ }^{116 \mathrm{~m}} \mathrm{In}$ via an $(n, \gamma)$ reaction. The observed interference with the $140 \mathrm{keV}$ peak is lower in Add Back mode than in Singles mode because one or more of them may be subject to TCS. Therefore, the detection limit is lower for Add Back mode for ${ }^{99 \mathrm{~m}} \mathrm{Tc}$ in these samples. Also due to the high ${ }^{235} \mathrm{U}$ content in the sample, the $740 \mathrm{keV}$ peak produces the lower detection limit for ${ }^{99} \mathrm{Mo}$ in Singles mode. The $182 \mathrm{keV}$ peak from ${ }^{235} \mathrm{U}$ interferes with the 181 keV peak from ${ }^{99} \mathrm{Mo}$, which raises the detection limit for this peak. 
Table 28: Detection limits in Bq for a 6-month old sample in Add Back mode.

Bolded values indicate the photon and counting mode that provide the lowest detection limits for the specific nuclide.

\begin{tabular}{|c|c|c|c|c|c|}
\hline \multirow{2}{*}{ Nuclide } & \multirow{2}{*}{$\begin{array}{c}\text { Peak Energy } \\
\text { (keV) }\end{array}$} & \multicolumn{4}{|c|}{ Counting Live Time } \\
\hline & & $60 \mathrm{~s}$ & $60 \mathrm{~min}$ & $24 \mathrm{~h}$ & $7 d$ \\
\hline${ }^{99 \mathrm{~m}} \mathrm{Tc} /{ }^{99} \mathrm{Mo}$ & 140 & 0.63 & 0.067 & 0.015 & 0.011 \\
\hline \multirow{3}{*}{${ }^{99} \mathrm{Mo}$} & 181 & 21.90 & 2.329 & 0.521 & 0.369 \\
\hline & 740 & 25.93 & 2.508 & 0.553 & 0.391 \\
\hline & 778 & 16.09 & 1.329 & 0.285 & 0.200 \\
\hline \multirow{4}{*}{${ }^{140} \mathrm{Ba}$} & 163 & 14.16 & 1.507 & 0.308 & 0.136 \\
\hline & 305 & 47.22 & 4.946 & 1.008 & 0.444 \\
\hline & 423 & 27.43 & 2.838 & 0.577 & 0.254 \\
\hline & 537 & 4.25 & 0.443 & 0.090 & 0.040 \\
\hline \multirow{4}{*}{${ }^{140} \mathrm{La}$} & 329 & 20.04 & 2.089 & 0.425 & 0.187 \\
\hline & 487 & 6.72 & 0.706 & 0.144 & 0.063 \\
\hline & 816 & 4.34 & 0.357 & 0.070 & 0.031 \\
\hline & 1596 & 2.55 & 0.178 & 0.034 & 0.015 \\
\hline \multirow{2}{*}{${ }^{95} \mathrm{Zr}$} & 724 & 2.16 & 0.209 & 0.041 & 0.016 \\
\hline & 756 & 1.56 & 0.143 & 0.028 & 0.011 \\
\hline$\frac{95}{{ }^{95}}$ & 765 & 0.85 & 0.078 & 0.015 & 0.006 \\
\hline
\end{tabular}


Table 29: Detection limits in Bq for a 6-month old sample in Singles mode.

Bolded values indicate the photon and counting mode that provide the lowest detection limits for the specific nuclide.

\begin{tabular}{|c|c|c|c|c|c|}
\hline \multirow{2}{*}{ Nuclide } & \multirow{2}{*}{$\begin{array}{c}\text { Peak Energy } \\
\text { (keV) }\end{array}$} & \multicolumn{4}{|c|}{ Counting Live Time } \\
\hline & & $60 \mathrm{~s}$ & $60 \mathrm{~min}$ & $24 \mathrm{~h}$ & $7 d$ \\
\hline${ }^{99 \mathrm{~m}} \mathrm{Tc} /{ }^{99} \mathrm{Mo}$ & 140 & 0.78 & 0.087 & 0.020 & 0.014 \\
\hline \multirow{3}{*}{${ }^{99} \mathrm{Mo}$} & 181 & 14.20 & 1.580 & 0.356 & 0.253 \\
\hline & 740 & 11.24 & 1.017 & 0.222 & 0.156 \\
\hline & 778 & 25.37 & 2.120 & 0.455 & 0.320 \\
\hline \multirow{4}{*}{${ }^{140} \mathrm{Ba}$} & 163 & 12.69 & 1.407 & 0.289 & 0.127 \\
\hline & 305 & 28.75 & 3.118 & 0.638 & 0.281 \\
\hline & 423 & 42.20 & 4.494 & 0.918 & 0.404 \\
\hline & 537 & 7.43 & 0.810 & 0.166 & 0.073 \\
\hline \multirow{4}{*}{${ }^{140} \mathrm{La}$} & 329 & 6.45 & 0.696 & 0.142 & 0.063 \\
\hline & 487 & 4.03 & 0.436 & 0.089 & 0.039 \\
\hline & 816 & 5.01 & 0.417 & 0.082 & 0.036 \\
\hline & 1596 & 1.78 & 0.125 & 0.024 & 0.010 \\
\hline \multirow{2}{*}{${ }^{95} \mathrm{Zr}$} & 724 & 2.76 & 0.250 & 0.049 & 0.019 \\
\hline & 756 & 2.13 & 0.185 & 0.036 & 0.014 \\
\hline${ }^{95} \mathrm{Nb}$ & 765 & 1.17 & 0.101 & 0.020 & 0.008 \\
\hline
\end{tabular}

\subsection{Detection Limits for One Month Old Sample}

A spectrum that is indicative of the count at around 30 days after zero time is from the 4130 sample. A spectrum was taken for 60,000 seconds 32.0 days after zero time. This spectrum had about $2.6 \%$ dead-time for all the observed peaks. As explained in Chapter 8 , different peaks can have different Dead-Times in Add Back mode due to the likelihood of interacting with more than one detector crystal. The dead-time will be taken into consideration, although the dead-time is expected to be much lower when waveform capture is turned off. The only peaks with interferences were the $141 \mathrm{keV}$ peak and the $163 \mathrm{keV}$ peak as explained previously. 
Table 30: Detection limits in Bq for a 1-month old sample in Add Back mode.

Bolded values indicate the photon and counting mode that provide the lowest detection limits for the specific nuclide.

\begin{tabular}{|c|c|c|c|c|c|}
\hline \multirow{2}{*}{ Nuclide } & $\begin{array}{c}\text { Peak Energy } \\
(\mathrm{keV})\end{array}$ & \multicolumn{4}{|c|}{ Counting Live Time } \\
\cline { 2 - 6 } & $\mathbf{1 4 0}$ & $\mathbf{1 . 5 4}$ & $\mathbf{0 . 1 8 5}$ & $\mathbf{0 . 0 4 2}$ & $\mathbf{0 . 0 3 0}$ \\
\hline${ }^{99 \mathrm{~m}} \mathrm{Tc} /{ }^{99} \mathrm{Mo}$ & 181 & 48.90 & 5.832 & 1.326 & 0.942 \\
\hline \multirow{3}{*}{${ }^{99} \mathrm{Mo}$} & 740 & 48.01 & 5.373 & 1.211 & 0.859 \\
\cline { 2 - 6 } & 778 & 34.39 & 3.703 & 0.830 & 0.589 \\
\cline { 2 - 6 } & 163 & 28.64 & 3.378 & 0.700 & 0.309 \\
\cline { 2 - 6 } & 305 & 98.98 & 11.636 & 2.409 & 1.063 \\
\cline { 2 - 6 } & 423 & 53.93 & 6.263 & 1.295 & 0.571 \\
\cline { 2 - 6 } & $\mathbf{5 3 7} \mathrm{Ba}$ & $\mathbf{7 . 8 3}$ & $\mathbf{0 . 9 0 7}$ & $\mathbf{0 . 1 8 7}$ & $\mathbf{0 . 0 8 3}$ \\
\hline \multirow{3}{*}{${ }^{140} \mathrm{La}$} & 329 & 41.29 & 4.835 & 1.001 & 0.442 \\
\cline { 2 - 6 } & 487 & 12.65 & 1.472 & 0.304 & 0.134 \\
\cline { 2 - 6 } & 816 & 9.07 & 0.968 & 0.198 & 0.087 \\
\cline { 2 - 6 } & 1596 & 6.40 & 0.676 & 0.138 & 0.061 \\
\hline \multirow{2}{*}{${ }^{95} \mathrm{Zr}$} & 724 & 4.08 & 0.456 & 0.092 & 0.036 \\
\hline${ }^{95} \mathrm{Nb}$ & $\mathbf{7 5 6}$ & $\mathbf{3 . 0 2}$ & $\mathbf{0 . 3 3 2}$ & $\mathbf{0 . 0 6 7}$ & $\mathbf{0 . 0 2 6}$ \\
\hline & $\mathbf{7 6 5}$ & $\mathbf{1 . 6 5}$ & $\mathbf{0 . 1 8 2}$ & $\mathbf{0 . 0 3 6}$ & $\mathbf{0 . 0 1 4}$ \\
\hline
\end{tabular}

Table 31: Detection limits for a 1-month old sample in Singles mode.

Bolded values indicate the photon and counting mode that provide the lowest detection limits for the specific nuclide.

\begin{tabular}{|c|c|c|c|c|c|}
\hline \multirow{2}{*}{ Nuclide } & $\begin{array}{c}\text { Peak Energy } \\
(\mathrm{keV})\end{array}$ & \multicolumn{4}{|c|}{ Counting Live Time } \\
\cline { 2 - 6 } & 140 & $60 \mathrm{~s}$ & $60 \mathrm{~min}$ & $24 \mathrm{~h}$ & $7 \mathrm{~d}$ \\
\hline${ }^{99 \mathrm{~m}} \mathrm{Tc} /{ }^{99} \mathrm{Mo}$ & 1.93 & 0.236 & 0.054 & 0.038 \\
\hline \multirow{3}{*}{${ }^{99} \mathrm{Mo}$} & 181 & 36.71 & 4.502 & 1.027 & 0.730 \\
\cline { 2 - 6 } & $\mathbf{7 4 0}$ & $\mathbf{2 3 . 0 5}$ & $\mathbf{2 . 5 4 9}$ & $\mathbf{0 . 5 7 4}$ & $\mathbf{0 . 4 0 7}$ \\
\cline { 2 - 6 } & 778 & 54.09 & 5.846 & 1.311 & 0.930 \\
\hline \multirow{3}{*}{${ }^{140} \mathrm{Ba}$} & 163 & 30.57 & 3.719 & 0.773 & 0.342 \\
\cline { 2 - 6 } & 305 & 70.56 & 8.521 & 1.770 & 0.782 \\
\cline { 2 - 6 } & 423 & 83.43 & 9.823 & 2.034 & 0.898 \\
\hline \multirow{3}{*}{${ }^{140} \mathrm{La}$} & 537 & 13.75 & 1.627 & 0.337 & 0.149 \\
\cline { 2 - 6 } & 329 & 14.97 & 1.796 & 0.373 & 0.165 \\
\cline { 2 - 6 } & 487 & 7.71 & 0.911 & 0.189 & 0.083 \\
\cline { 2 - 6 } & $\mathbf{1 5 9 6}$ & 10.50 & 1.126 & 0.230 & 0.101 \\
\hline \multirow{2}{*}{${ }^{95} \mathrm{Zr}$} & 724 & 5.16 & $\mathbf{0 . 3 0 4}$ & $\mathbf{0 . 0 6 1}$ & $\mathbf{0 . 0 2 7}$ \\
\hline${ }^{95} \mathrm{Nb}$ & 756 & 4.43 & 0.638 & 0.128 & 0.050 \\
\hline
\end{tabular}




\subsection{Detection Limit for Four Day Old Sample}

The youngest spectrum is taken from the 4130 sample. The spectrum was taken for 6,000 seconds "Live Time" and started when the sample was 4.5 days old. There is significant dead-time that will be taken into account in this analysis. This dead-time varies significantly as a function of energy as described in Chapter 8. In this spectrum, the $141 \mathrm{keV}$ peak sits on a downward slope and is surrounded by several peaks on either side. For the sake of simplicity, the underlying continuum for this peak was observed below $141 \mathrm{keV}$, and it appears to be higher than the continuum that the peak actually sits on. Therefore, the detection limits shown in these tables for $141 \mathrm{keV}$ are a conservative estimate of the true detection limit for the 141 keV peak. In actuality, the detection limits are probably much lower, but more accurate values are more difficult to calculate.

Table 32: Detection limits for a 4-day old sample in Add Back mode.

Bolded values indicate the photon and counting mode that provide the lowest detection limits for the specific nuclide.

\begin{tabular}{|c|c|c|c|c|c|}
\hline \multirow{2}{*}{ Nuclide } & $\begin{array}{c}\text { Peak Energy } \\
(\mathrm{keV})\end{array}$ & \multicolumn{4}{|c|}{ Add Back Mode Detection Limit (Bq) } \\
\cline { 2 - 6 } & $\mathbf{1 4 0}$ & $\mathbf{5 . 2 1}$ & $\mathbf{0 . 6 6 1}$ & $\mathbf{0 . 1 5 1}$ & $\mathbf{0 . 1 0 8}$ \\
\hline${ }^{99 \mathrm{~m}} \mathrm{Tc} /{ }^{99} \mathrm{Mo}$ & $\mathbf{1 4 0}$ & 135.17 & 17.027 & 3.898 & 2.772 \\
\hline \multirow{3}{*}{${ }^{99} \mathrm{Mo}$} & 181 & 127.07 & 15.632 & 3.568 & 2.537 \\
\cline { 2 - 6 } & 740 & 104.27 & 12.772 & 2.914 & 2.071 \\
\cline { 2 - 6 } & 778 & 83.03 & 10.408 & 2.172 & 0.960 \\
\hline \multirow{3}{*}{${ }^{140} \mathrm{Ba}$} & 163 & 226.66 & 28.137 & 5.866 & 2.592 \\
\cline { 2 - 6 } & 305 & 126.89 & 15.693 & 3.270 & 1.445 \\
\cline { 2 - 6 } & 423 & $\mathbf{1 8 . 6 3}$ & $\mathbf{2 . 3 0 2}$ & $\mathbf{0 . 4 8 0}$ & $\mathbf{0 . 2 1 2}$ \\
\hline \multirow{3}{*}{${ }^{140} \mathrm{La}$} & $\mathbf{5 3 7}$ & 98.13 & 12.182 & 2.540 & 1.122 \\
\cline { 2 - 6 } & 329 & 29.86 & 3.697 & 0.770 & 0.340 \\
\cline { 2 - 6 } & 487 & 28.56 & 3.487 & 0.725 & 0.320 \\
\cline { 2 - 6 } & 1596 & 16.57 & 1.991 & 0.413 & 0.183 \\
\hline \multirow{2}{*}{${ }^{95} \mathrm{Zr}$} & $\mathbf{7 2 4}$ & 10.55 & 1.292 & 0.263 & 0.103 \\
\cline { 2 - 6 } & $\mathbf{7 5 6}$ & $\mathbf{8 . 4 6}$ & $\mathbf{1 . 0 3 4}$ & $\mathbf{0 . 2 1 1}$ & $\mathbf{0 . 0 8 2}$ \\
\hline${ }^{95} \mathrm{Nb}$ & $\mathbf{7 6 5}$ & $\mathbf{4 . 6 3}$ & $\mathbf{0 . 5 6 6}$ & $\mathbf{0 . 1 1 5}$ & $\mathbf{0 . 0 4 5}$ \\
\hline
\end{tabular}


Table 33: Detection limits for a 4-day old sample in Singles mode.

Bolded values indicate the photon and counting mode that provide the lowest detection limits for the specific nuclide.

\begin{tabular}{|c|c|c|c|c|c|}
\hline \multirow{2}{*}{ Nuclide } & $\begin{array}{c}\text { Peak Energy } \\
(\mathrm{keV})\end{array}$ & \multicolumn{4}{|c|}{ Singles Mode Detection Limit (Bq) } \\
\cline { 2 - 6 } & 140 & $60 \mathrm{~s}$ & $60 \mathrm{~min}$ & $24 \mathrm{~h}$ & $7 \mathrm{~d}$ \\
\hline${ }^{99 \mathrm{~m}} \mathrm{Tc} /{ }^{99} \mathrm{Mo}$ & 6.45 & 0.823 & 0.189 & 0.134 \\
\hline \multirow{3}{*}{$99 \mathrm{Mo}$} & 181 & 101.79 & 12.947 & 2.967 & 2.111 \\
\cline { 2 - 6 } & $\mathbf{7 4 0}$ & $\mathbf{6 2 . 7 4}$ & $\mathbf{7 . 7 0 1}$ & $\mathbf{1 . 7 5 7}$ & $\mathbf{1 . 2 4 9}$ \\
\cline { 2 - 6 } & 778 & 149.41 & 18.216 & 4.153 & 2.952 \\
\hline \multirow{3}{*}{${ }^{140} \mathrm{Ba}$} & 163 & 89.74 & 11.366 & 2.375 & 1.050 \\
\cline { 2 - 6 } & 305 & 176.87 & 22.261 & 4.649 & 2.055 \\
\cline { 2 - 6 } & 423 & 244.95 & 30.699 & 6.407 & 2.832 \\
\cline { 2 - 6 } & 537 & 36.47 & 4.563 & 0.952 & 0.421 \\
\hline \multirow{3}{*}{${ }^{140} \mathrm{La}$} & 329 & 39.48 & 4.965 & 1.037 & 0.458 \\
\cline { 2 - 6 } & 487 & 21.84 & 2.738 & 0.571 & 0.253 \\
\cline { 2 - 6 } & 816 & 28.57 & 3.462 & 0.719 & 0.318 \\
\hline \multirow{2}{*}{${ }^{95} \mathrm{Zr}$} & $\mathbf{1 5 9 6}$ & $\mathbf{6 . 5 8}$ & $\mathbf{0 . 7 4 6}$ & $\mathbf{0 . 1 5 4}$ & $\mathbf{0 . 0 6 8}$ \\
\cline { 2 - 6 } & 724 & 15.45 & 1.889 & 0.385 & 0.150 \\
\hline${ }^{95} \mathrm{Nb}$ & 756 & 12.22 & 1.489 & 0.303 & 0.118 \\
\hline
\end{tabular}

\subsection{Comparison of Detection Limits with Traditional System}

A traditional system generally consists of one HPGe crystal. This can be simulated with the clover system at LANL by simply observing spectra from one of the HPGe crystals. To show the improvements in the detection limits for this system compared with a traditional system, Table 34 shows the detection limits for the system as a whole compared with just one of the HPGe crystals for a 60 minute count time. As indicated in the Table, the detection limits are improved by a factor of 2.8 to 5.5 when compared with a traditional gamma detection system. 
Table 34: Comparison of Detection Limits with Traditional System for 60 min Count Time

\begin{tabular}{|c|c|c|c|}
\hline Nuclide (Energy keV) & Sample Type & $\begin{array}{c}\text { Full System Detection } \\
\text { Limit (Bq) }\end{array}$ & $\begin{array}{c}\text { One Crystal Detection } \\
\text { Limit (Bq) }\end{array}$ \\
\hline \multirow{4}{*}{${ }^{99 m} \mathrm{Tc}(140)$} & Near Background & 0.032 & 0.099 \\
\hline & 6 Month Old & 0.067 & 0.256 \\
\hline & 1 Month Old & 0.185 & 0.678 \\
\hline & 4 Day Old & 0.661 & 2.338 \\
\hline \multirow{4}{*}{${ }^{99} \mathrm{Mo}(740)$} & Near Background & 0.608 & 2.059 \\
\hline & 6 Month Old & 1.017 & 3.216 \\
\hline & 1 Month Old & 2.549 & 7.548 \\
\hline & 4 Day Old & 7.701 & 22.12 \\
\hline \multirow{4}{*}{${ }^{140} \mathrm{Ba}(537)$} & Near Background & 0.162 & 0.771 \\
\hline & 6 Month Old & 0.443 & 2.407 \\
\hline & 1 Month Old & 0.907 & 4.716 \\
\hline & 4 Day Old & 2.302 & 13.021 \\
\hline \multirow{4}{*}{${ }^{140} \mathrm{La}(1596)$} & Near Background & 0.101 & 0.367 \\
\hline & 6 Month Old & 0.125 & 0.433 \\
\hline & 1 Month Old & 0.304 & 0.940 \\
\hline & 4 Day Old & 0.746 & 2.190 \\
\hline \multirow{4}{*}{${ }^{95} \mathrm{Zr}(756)$} & Near Background & 0.079 & 0.411 \\
\hline & 6 Month Old & 0.143 & 0.591 \\
\hline & 1 Month Old & 0.332 & 1.433 \\
\hline & 4 Day Old & 1.034 & 4.279 \\
\hline \multirow{4}{*}{${ }^{95} \mathrm{Nb}(765)$} & Near Background & 0.043 & 0.226 \\
\hline & 6 Month Old & 0.078 & 0.325 \\
\hline & 1 Month Old & 0.182 & 0.787 \\
\hline & 4 Day Old & 0.566 & 2.349 \\
\hline
\end{tabular}




\section{Part 5: Conclusions}

\section{Chapter 13: Work Performed}

The clover detector system at Los Alamos National Laboratory has had many settings optimally initialized and thoroughly characterized. The sensitivity of peak resolution has been observed as a function of the shaping parameters, which include the Energy Rise Time and the Energy Flat Top. A routine has been set up that ensures the energy gain in units of keV per channel is set to 0.09155 , which ensures the energy range is exactly $3000 \mathrm{keV}$ for each spectrum. This ensures the optimal resolution is attained for combined spectra, which includes Add Back mode, Singles mode, and gamma-gamma coincidence mode. The low-energy threshold was set so noise was not appreciably allowed in the low-energy portion of the spectrum. This threshold depends on the Threshold, Trigger Rise Time, and Trigger Flat Top settings. These settings have been explored and initially set based on the noise observed in the system. Other settings have also been optimized for the system to be used in List Mode to record data with the highest quality possible.

The system has been characterized in terms of peak-to-total ratios, efficiency curves, and the effects of dead-time on the system. The peak-to-total ratios were obtained with (mostly) single photon emitters ranging in energy from $53 \mathrm{keV}$ to $1165 \mathrm{keV}$. Efficiency curves for a Type 1 (point) source and a Type 3 (disk) source were obtained for all counting modes, including for use with gamma-gamma coincidence mode. The dead-time effects on the system were explored and the differences between analog systems and digital systems were pointed out, as well as the differences between single-crystal detectors and multi-crystal detectors. Among the chief differences are the effects of waveform collection on the dead-time as well as the dependence of dead-time on the energy of the photon of interest in certain counting modes.

Once the system was characterized, the application to nuclear forensics was emphasized by analyzing irradiated uranium samples over a period of five months. Two different samples were analyzed for three different fission product pairs: ${ }^{99} \mathrm{Mo} /{ }^{99 \mathrm{~m}} \mathrm{Tc},{ }^{140} \mathrm{Ba} /{ }^{140} \mathrm{La}$, and ${ }^{95} \mathrm{Zr} /{ }^{95} \mathrm{Nb}$. The decay schemes for each of these nuclides containing the relevant gamma-ray energies and intensities as well as the half-life of each nuclide can be found in Appendix 4. The 
half-lives for these pairs are very different and emphasize the ability of the system to analyze samples of various ages. The gamma-ray emissions for these fission products enlighten the various strengths and weaknesses of each counting mode - specifically in regard to singlet gamma-ray emissions versus cascading gamma-ray emissions. The activity of each fission product was calculated at for each spectrum and the ability of the system to provide age dating analysis on the samples was demonstrated. The detection limits of the system were also explored for four sample types: a near background sample, a young sample less than one week old, a middle-aged sample about one month old, and an older sample about five months old.

This system is capable of handling a variety of sample types, sample geometries, and nuclides, allowing this system to be used effectively in analyzing nuclear forensics samples. Properties such as sample age and fission product activity can be well-defined, which are useful in the attribution process of law-enforcement officials.

This detector system is capable of significantly enhancing nuclear forensic property determinations compared to traditional systems. By initializing system settings and characterizing the system in general, the abilities that researchers have for analyzing nuclear forensics samples is unprecedented. The procedure laid out in this work to initialize settings and characterize a detector system for use with nuclear forensics samples has not been shown before. The analysis of dead-time with respect to digitizing systems is also a unique contribution of this work. Nowhere in the literature has dead-time been thoroughly explored in multidetector systems. The usage of gamma-gamma coincidence mode is also not well-utilized and gives analysts great potential in isolating cascading gamma-ray peaks in the midst of interferences and high background - especially in fission products such as ${ }^{140}$ La. The high efficiency of this system allows for researchers to utilize gamma-gamma coincidence mode to a greater extent than traditional gamma-ray detection systems have previously allowed. The detection limits shown for this system are unattainable in most other systems due to high background or low sensitivity. The ability for researchers to detect and quantify low-levels of radiation gives rise to more detailed forensics analysis capabilities that are not possible with existing systems. 


\section{Chapter 14: Future Work}

The Trigger Rise Time and Trigger Flat Top settings in conjunction with the Threshold setting should be further explored. It is surmised that varying the Trigger Flat Top can vary the "sharpness" of the low-energy threshold cliff. This is a desirable attribute because the goal is to cut out the low-energy noise completely and obtain all of the events above the threshold. This needs to be explored further. The Energy Flat Top should also be looked into further, as the optimal value for this setting is the width of the rising edge of the pulse with the highest expected energy. The highest energy expected at this point is around 3,000 keV, so being able to observe events with this energy to set the Energy Flat Top would be optimal.

The characterization of the system could be improved by obtaining a peak-to-total ratio curve using the optimal settings. Mainly, the peak-to-total samples should be recounted with the waveform capture function turned off. This will reduce dead-time effects in the system and will provide a more accurate peak-to-total ratio curve. Optimally, the peak-to-total ratio samples will also be of the same geometry as the samples that the system is expected to observe. It has been shown that the placement of the sample will not greatly affect the peak-tototal ratio of the photons being emitted from that sample [43], but this was only shown for single detector systems without scattering materials in the vicinity of the detector system. This setup involves multiple detector crystals and a large amount of scattering material. The effect of the location within the detector system on the peak-to-total ratios should be explored. Another factor that can contribute to errors in the results is the effects produced by the $\beta^{-}$ particles in the $\beta^{-}$decays. The effects of $\beta^{-}$particles that penetrate the aluminum end-cap or cause bremsstrahlung radiation in the system should be explored. A good way to do this would be to observe $\beta^{-}$emitters that do not emit any gamma-rays.

The efficiency curves should also be observed again with the waveform capture turned off. As it stands, the efficiency curves have been corrected for dead-time effects, but these corrections are only approximate. By turning off the waveform capture, the dead-time will be reduced dramatically, and the efficiency curves can be determined more accurately. Efficiency curves of other sample geometries should also be explored, as well as the effect of location within the detector system on the efficiency curves for the various counting modes. As of now, the detectors are kept less than one $\mathrm{cm}$ apart from each other, but some samples are thicker 
than one $\mathrm{cm}$. Therefore, efficiency curves and peak-to-total ratios should also be obtained for different detector separation distances.

There is an internal setting in the system that keeps track of dead-time, input count rates, output count rates, and a variety of other useful features. It would be optimal if the sample counting program could output a file containing the different "attributes" of the count that the system automatically calculates. This type of a file would be helpful if the energy calibration file gets lost, if the count is stopped unexpectedly, or a variety of other conceivable problems that would be difficult to correct for at this point.

Of particular interest to nuclear forensics problems would be observing a spectrum that is closer to zero time to observe the effects of the short-lived fission products on the spectrum. Once the peak-to-total ratio curve has been better established, the spectra should also be reanalyzed for better defining the activities derived from the cascading gamma-rays. It is expected that gamma-gamma coincidence mode will also provide better data once the peak-tototal ratio curves and dead-time effects on the spectrum have been further explored.

The value of Compton-suppressed mode should be looked into further for nuclear forensics samples. Specifically, Compton-suppressed mode can lower the detection limits for singlet gamma-ray emissions that occur in the low-energy Compton continuum. By lowering this continuum, the overall background for these peaks will be lowered and can lessen interferences between some peaks.

The detection limits should also be analyzed more thoroughly. As it stands, the detection limits are calculated by observing the major interference peaks and the background counts close to the peak of interest. In some cases, the background was treated as a straight line or step-function when it could have been defined by a better function such as a quadratic or smoothed step function. The detection limits for a wider variety of photon energies could also have been obtained. Once the gamma-gamma coincidence mode spectra have been shown to produce more accurate results, the detection limits should also be looked into for this mode, especially for nuclides in the presence of interfering peaks. Finally, the potential applicability of the clover system to analyzing nuclear forensics samples should be expanded to include more nuclides. The addition of other nuclides could potentially provide more information as to the 
origin and original composition of the sample. Having the ability to analyze a wide set of nuclides would make the clover system invaluable to the nuclear forensics community 


\section{Appendix 1: Uncertainty Calculations for Best-Fit Equations}

The following information is borrowed heavily from a paper by Edgeworth [45]. Best-fit equations are found by minimizing $\chi^{2}$ with respect to the coefficients being found. $\chi^{2}$ is given by:

$$
\chi^{2}=\sum_{i=1}^{n}\left[\frac{y_{i}-\hat{y}_{i}}{\sigma_{i}}\right]^{2}
$$

where:

$\hat{y}_{i}$ is the predicted value of the $i^{\text {th }}$ data point

$y_{i}$ is the measured value of the $i^{\text {th }}$ data point

$\sigma_{i}$ is the uncertainty of the measured value in the $i^{\text {th }}$ data point.

In general, the uncertainty in a best fit curve is a function of the uncertainties in the coefficients and the covariances between the coefficients. This is given by the variancecovariance matrix, which can be estimated by inverting the Fisher matrix (we assume our model is a valid way of interpreting the data). The general form for each element in the Fisher matrix, C, is given by:

$$
C_{j k}=\sum_{i=1}^{n} w_{i} \frac{\partial f}{\partial a_{j}} \frac{\partial f}{\partial a_{k}}
$$

where:

$n$ is the number of points used to obtain the fit

$a_{m}$ is the $m^{\text {th }}$ constant in $f$

$w_{i}$ is the weight given to the $i^{\text {th }}$ point

Each of the partial derivatives is evaluated at $i$ in the summation. There are a number of different weighting schemes, but for this work the weight will be the inverse of the square of the uncertainty at the point $i$ :

$$
w_{i}=\frac{1}{\sigma_{i}^{2}}
$$

Substituting Equation (A1.3) into (A1.2), the Fisher matrix becomes:

$$
C_{j k}=\sum_{i=1}^{n} \frac{1}{{\sigma_{i}}^{2}} \frac{\partial f}{\partial a_{j}} \frac{\partial f}{\partial a_{k}}
$$


Inverting this gives us the variance-covariance matrix for the fitted peak:

$$
V=C^{-1}
$$

where:

$\mathbf{V}$ is the variance-covariance matrix

$\mathbf{C}$ is the Fisher matrix

V will give the variances and covariances of the parameters in the following form:

$$
\boldsymbol{V}=\left[\begin{array}{cccc}
\sigma_{0}{ }^{2} & \rho_{0,1} \sigma_{0} \sigma_{1} & \cdots & \rho_{0, n} \sigma_{0} \sigma_{n} \\
& & & \\
\rho_{1,0} \sigma_{1} \sigma_{0} & \sigma_{1}{ }^{2} & \cdots & \rho_{1, n} \sigma_{1} \sigma_{n} \\
\vdots & \vdots & \ddots & \vdots \\
\rho_{n, 0} \sigma_{n} \sigma_{0} & \rho_{n, 1} \sigma_{n} \sigma_{1} & \cdots & \sigma_{n}{ }^{2}
\end{array}\right]
$$

where:

$\rho_{i, j}$ is the correlation between the $i^{\text {th }}$ and $j^{\text {th }}$ coefficient

$\sigma_{i}^{2}$ is the variance in the $i^{\text {th }}$ coefficient

The variances in each parameter are given by the diagonals of $\mathbf{V}$, however, the correlations between the coefficients are non-zero. Therefore, the entire variance-covariance matrix must be used when calculating the uncertainty of the equation of best fit. To calculate the uncertainty of an estimated value using the best fit equation, the partial derivatives of the best fit function with respect to each of the coefficients must be found. This is represented by:

$$
A=\left(\begin{array}{c}
\frac{\partial f}{\partial a_{0}} \\
\frac{\partial f}{\partial a_{1}} \\
\vdots
\end{array}\right)
$$

where each partial derivative is evaluated at the specified bin. The uncertainty of the estimated value can then be given by:

$$
\sigma(f(E))=\left[A^{T} V A\right]^{1 / 2}
$$

$A^{T}$ is a $1 \times n$ matrix, $V$ is a $n \times n$ matrix, and $A$ is a $n \times 1$ matrix, so $A^{T} V A$ will be $1 \times 1$. This takes all variances and covariances into account with the data. 


\section{Appendix 2: Example Parameter File}

The following lines are indicative of a typical input parameter file. In each line, the first word represents the name of the variable. The second word represents whether the variable is a Channel parameter or a Module parameter. The third entry represents the module number that the input applies to. The fourth entry represents the channel number that the input applies to. If the input is a module parameter, then the fourth entry is ignored. The fifth entry is the value for the input parameter.

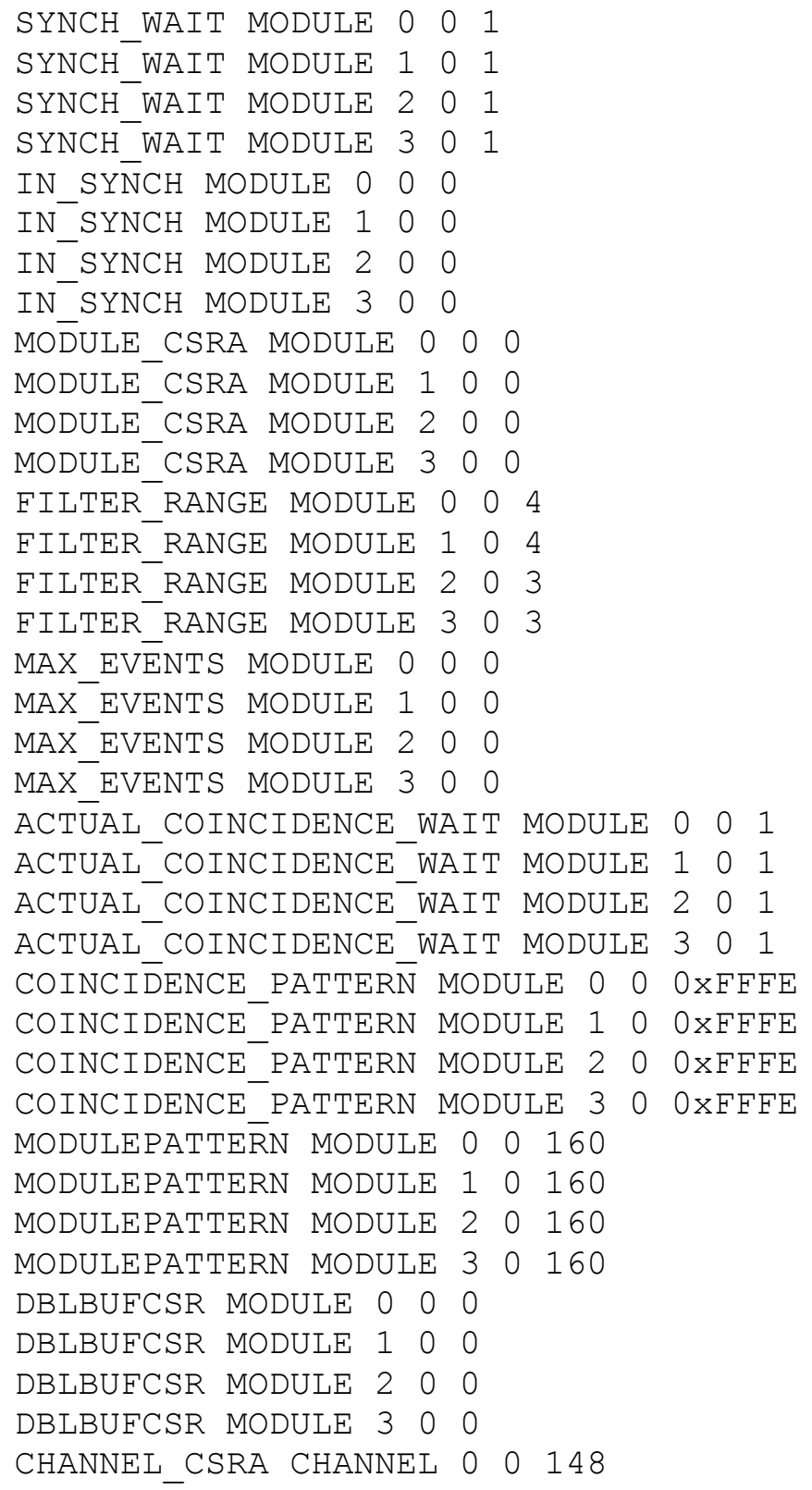


CHANNEL CSRA CHANNEL O 1148

CHANNEL CSRA CHANNEL O 2148

CHANNEL_CSRA CHANNEL 030148

CHANNEL_CSRA CHANNEL 100148

CHANNEL_CSRA CHANNEL 11148

CHANNEL_CSRA CHANNEL 122148

CHANNEL_CSRA CHANNEL 133148

CHANNEL_CSRA CHANNEL 200148

CHANNEL_CSRA CHANNEL $2 \quad 1 \quad 148$

CHANNEL_CSRA CHANNEL 222148

CHANNEL_CSRA CHANNEL $2 \quad 3 \quad 148$

CHANNEL_CSRA CHANNEL 300148

CHANNEL_CSRA CHANNEL $3 \quad 1 \quad 148$

CHANNEL_CSRA CHANNEL 322148

CHANNEL_CSRA CHANNEL $3 \quad 3 \quad 148$

TRIGGER_RISETIME CHANNEL 0000.080

TRIGGER_RISETIME CHANNEL 0010.080

TRIGGER_RISETIME CHANNEL 0220.080

TRIGGER_RISETIME CHANNEL 030.080

TRIGGER_RISETIME CHANNEL 100.080

TRIGGER_RISETIME CHANNEL 1110.080

TRIGGER_RISETIME CHANNEL 1220.080

TRIGGER_RISETIME CHANNEL 1330.080

TRIGGER_RISETIME CHANNEL $2 \quad 0 \quad 0.080$

TRIGGER_RISETIME CHANNEL $2 \quad 1 \quad 0.080$

TRIGGER_RISETIME CHANNEL 2220.080

TRIGGER_RISETIME CHANNEL $2 \quad 3 \quad 0.080$

TRIGGER_RISETIME CHANNEL $3 \quad 0 \quad 0.080$

TRIGGER_RISETIME CHANNEL $3 \quad 1 \quad 0.080$

TRIGGER_RISETIME CHANNEL 3220.080

TRIGGER_RISETIME CHANNEL $3 \quad 3 \quad 0.080$

TRIGGER FLATTOP CHANNEL 0000.080

TRIGGER_FLATTOP CHANNEL 010.080

TRIGGER_FLATTOP CHANNEL 0200.080

TRIGGER FLATTOP CHANNEL 030.080

TRIGGER_FLATTOP CHANNEL $1 \quad 0 \quad 0.080$

TRIGGER_FLATTOP CHANNEL 110.080

TRIGGER FLATTOP CHANNEL 120.080

TRIGGER_FLATTOP CHANNEL 130.080

TRIGGER_FLATTOP CHANNEL 2002.5

TRIGGER_FLATTOP CHANNEL $2 \quad 1 \quad 2.5$

TRIGGER_FLATTOP CHANNEL $222 \quad 2.5$

TRIGGER FLATTOP CHANNEL $2 \quad 3 \quad 2.5$

$\begin{array}{llllll}\text { TRIGGER_ELATTOP CHANNEL } & 3 & 0 & 2.5\end{array}$

TRIGGER_FLATTOP CHANNEL $3 \quad 1 \quad 2.5$

$\begin{array}{lllll}\text { TRIGGER FLATTOP CHANNEL } & 3 & 2 & 2.5\end{array}$

$\begin{array}{llllll} & \text { TRIGGER_ELATTOP CHANNEL } & 3 & 2.5\end{array}$

TRIGGER THRESHOLD CHANNEL 00012 .

TRIGGER_THRESHOLD CHANNEL 0112 . 
TRIGGER THRESHOLD CHANNEL 02212 . TRIGGER THRESHOLD CHANNEL $0 \quad 3 \quad 12$. TRIGGER THRESHOLD CHANNEL 10012. TRIGGER THRESHOLD CHANNEL 1112 . TRIGGER THRESHOLD CHANNEL 12212 . TRIGGER THRESHOLD CHANNEL 1312 . TRIGGER THRESHOLD CHANNEL 20025. TRIGGER THRESHOLD CHANNEL 21125. TRIGGER THRESHOLD CHANNEL 22225. TRIGGER THRESHOLD CHANNEL 2325. TRIGGER THRESHOLD CHANNEL 30025. TRIGGER THRESHOLD CHANNEL 31125. TRIGGER THRESHOLD CHANNEL 32225. TRIGGER THRESHOLD CHANNEL 3325. ENERGY RISETIME CHANNEL 00012.0 ENERGY RISETIME CHANNEL 00112.0 ENERGY RISETIME CHANNEL $002 \quad 12.0$ ENERGY RISETIME CHANNEL $003 \quad 12.0$ ENERGY RISETIME CHANNEL 1 C 12.0 ENERGY RISETIME CHANNEL $1 \quad 1 \quad 12.0$ ENERGY RISETIME CHANNEL $1212 \quad 12.0$ ENERGY RISETIME CHANNEL 13012.0 ENERGY RISETIME CHANNEL $2 \quad 0 \quad 0.0$ ENERGY RISETIME CHANNEL $2 \quad 1 \quad 0.0$ ENERGY RISETIME CHANNEL $22 \quad 2 \quad 0.0$ ENERGY RISETIME CHANNEL 230.0 ENERGY RISETIME CHANNEL $3 \quad 0 \quad 0.0$ ENERGY RISETIME CHANNEL $3 \quad 1 \quad 0.0$ ENERGY RISETIME CHANNEL $3 \quad 2 \quad 0.0$ ENERGY RISETIME CHANNEL $3 \quad 3 \quad 0.0$ ENERGY FLATTOP CHANNEL 000 ENERGY FLATTOP CHANNEL 0011.2 ENERGY_FLATTOP CHANNEL $002 \quad 1.2$ ENERGY_FLATTOP CHANNEL 0031.2 ENERGY FLATTOP CHANNEL 11001.2

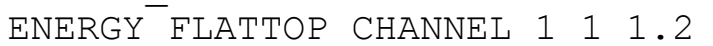
ENERGY_FLATTOP CHANNEL 12 ENERGY FLATTOP CHANNEL 1331.2

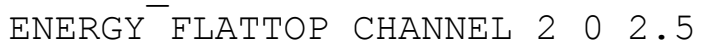
ENERGY FLATTOP CHANNEL $2 \quad 1 \quad 2.5$ ENERGY FLATTOP CHANNEL $2 \quad 2 \quad 2.5$

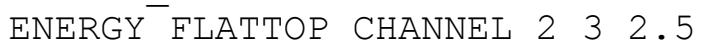
ENERGY FLATTOP CHANNEL $300 \quad 2.5$ $\begin{array}{lllll}\text { ENERGY FLATTOP CHANNEL } & 3 & 1 & 2.5\end{array}$ $\begin{array}{lllll}\text { ENERGY FLATTOP CHANNEL } & 3 & 2 & 2.5\end{array}$ ENERGY FLATTOP CHANNEL $3 \quad 3 \quad 2.5$

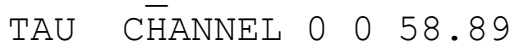

TAU CHANNEL $0 \quad 1 \quad 57.41$

TAU CHANNEL $0 \quad 2 \quad 56.04$ 


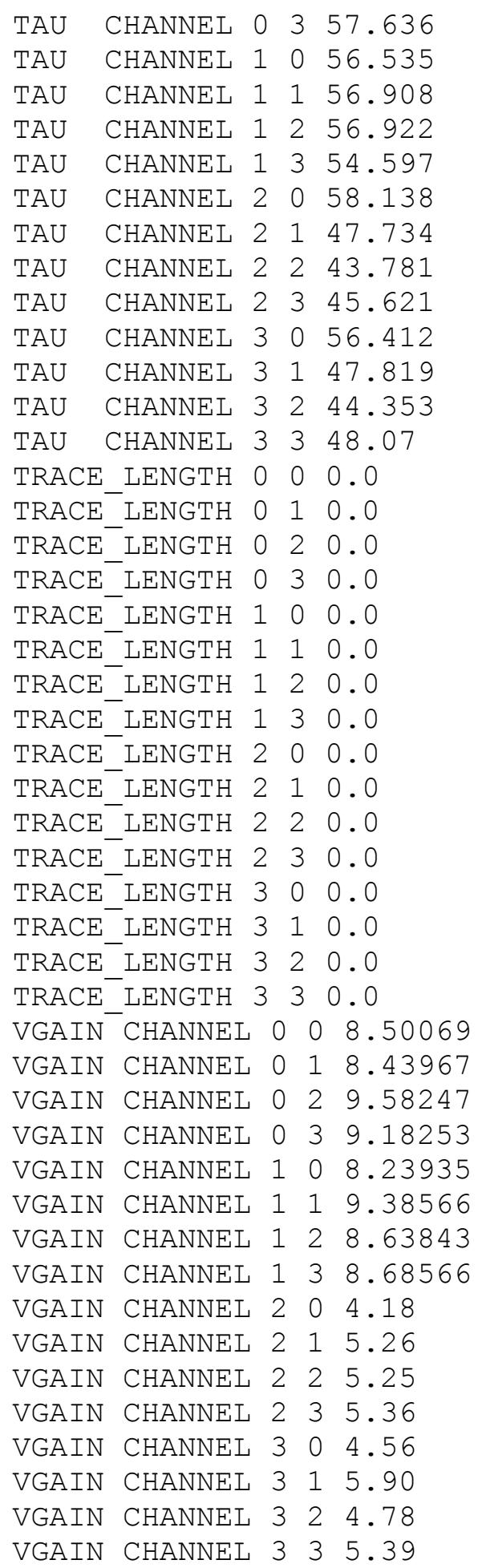




\section{Appendix 3: P/T Ratio and Efficiency Curve Data}

The peak-to-total ratio data and efficiency curve data for each counting mode are given in the following Tables for reference. They have been calculated according to the methods described in Chapter 6 and Chapter 7.

Table 35: Coefficients for $\mathrm{P} / \mathrm{T}$ ratio equations of best fit for relevant counting modes.

\begin{tabular}{|c|c|c|}
\hline Coefficient & Add Back & Singles \\
\hline$a_{0}$ & -1.684 & -5.742 \\
\hline$a_{1}$ & 0.653 & 2.494 \\
\hline$a_{2}$ & -0.0232 & -0.117 \\
\hline$a_{3}$ & -0.0173 & -0.0649 \\
\hline$a_{4}$ & 0.00120 & 0.00592 \\
\hline$a_{5}$ & 2.505 & 3.061 \\
\hline$a_{6}$ & -0.543 & -0.729 \\
\hline
\end{tabular}

Table 36: Peak Efficiency for Type 3 (Disk) Source all counting modes

\begin{tabular}{|c|c|c|c|c|}
\hline \multirow{2}{*}{ Energy (keV) } & \multicolumn{4}{|c|}{ Corrected Efficiency } \\
\cline { 2 - 5 } & Add Back & Singles & Add Back Misty & Add Back Kerri \\
\hline 59.54 & $0.477 \pm 0.009$ & $0.473 \pm 0.009$ & $0.263 \pm 0.005$ & $0.210 \pm 0.004$ \\
\hline 88.0336 & $0.513 \pm 0.018$ & $0.510 \pm 0.018$ & $0.276 \pm 0.010$ & $0.234 \pm 0.008$ \\
\hline 122.06 & $0.497 \pm 0.014$ & $0.486 \pm 0.014$ & $0.263 \pm 0.008$ & $0.227 \pm 0.007$ \\
\hline 165.864 & $0.470 \pm 0.012$ & $0.430 \pm 0.011$ & $0.239 \pm 0.006$ & $0.206 \pm 0.005$ \\
\hline 279.1967 & $0.321 \pm 0.025$ & $0.292 \pm 0.013$ & $0.176 \pm 0.009$ & $0.164 \pm 0.016$ \\
\hline 391.69 & $0.281 \pm 0.007$ & $0.211 \pm 0.005$ & $0.137 \pm 0.003$ & $0.122 \pm 0.003$ \\
\hline 661.66 & $0.200 \pm 0.006$ & $0.133 \pm 0.004$ & $0.0956 \pm 0.0028$ & $0.0855 \pm 0.0025$ \\
\hline 898.042 & $0.168 \pm 0.007$ & $0.109 \pm 0.003$ & $0.0835 \pm 0.0029$ & $0.0720 \pm 0.0024$ \\
\hline 1173.237 & $0.143 \pm 0.005$ & $0.0856 \pm 0.0025$ & $0.0678 \pm 0.0023$ & $0.0594 \pm 0.0019$ \\
\hline 1332.501 & $0.131 \pm 0.005$ & $0.0770 \pm 0.0022$ & $0.0617 \pm 0.0020$ & $0.0542 \pm 0.0017$ \\
\hline 1836.063 & $0.104 \pm 0.004$ & $0.0600 \pm 0.0017$ & $0.0496 \pm 0.0015$ & $0.0427 \pm 0.0013$ \\
\hline
\end{tabular}


Table 37: Peak Efficiency for Type 1 (Point) Source all counting modes

\begin{tabular}{|c|c|c|c|c|}
\hline \multirow{2}{*}{ Energy (keV) } & \multicolumn{4}{|c|}{ Corrected Efficiency } \\
\cline { 2 - 5 } & Add Back & Singles & Add Back Misty & Add Back Kerri \\
\hline 59.54 & $0.469 \pm 0.016$ & $0.469 \pm 0.016$ & $0.262 \pm 0.009$ & $0.208 \pm 0.007$ \\
\hline 88.0336 & $0.518 \pm 0.024$ & $0.515 \pm 0.024$ & $0.281 \pm 0.013$ & $0.235 \pm 0.011$ \\
\hline 122.06 & $0.502 \pm 0.020$ & $0.491 \pm 0.020$ & $0.267 \pm 0.011$ & $0.226 \pm 0.009$ \\
\hline 165.864 & $0.481 \pm 0.019$ & $0.388 \pm 0.015$ & $0.242 \pm 0.010$ & $0.209 \pm 0.008$ \\
\hline 279.1967 & $0.359 \pm 0.015$ & $0.283 \pm 0.012$ & $0.186 \pm 0.008$ & $0.164 \pm 0.007$ \\
\hline 391.69 & $0.286 \pm 0.011$ & $0.215 \pm 0.008$ & $0.142 \pm 0.006$ & $0.123 \pm 0.005$ \\
\hline 661.66 & $0.205 \pm 0.008$ & $0.136 \pm 0.005$ & $0.0996 \pm 0.0040$ & $0.0862 \pm 0.0034$ \\
\hline 898.042 & $0.178 \pm 0.007$ & $0.0999 \pm 0.0039$ & $0.0869 \pm 0.0035$ & $0.0731 \pm 0.0029$ \\
\hline 1173.237 & $0.150 \pm 0.007$ & $0.0772 \pm 0.0030$ & $0.0710 \pm 0.0028$ & $0.0603 \pm 0.0024$ \\
\hline 1332.501 & $0.138 \pm 0.006$ & $0.0703 \pm 0.0027$ & $0.0648 \pm 0.0026$ & $0.0548 \pm 0.0022$ \\
\hline 1836.063 & $0.111 \pm 0.004$ & $0.0551 \pm 0.0022$ & $0.0513 \pm 0.0020$ & $0.0435 \pm 0.0017$ \\
\hline
\end{tabular}

Table 38: Coefficients for efficiency equations for Type 3 source

\begin{tabular}{|c|c|c|c|c|}
\hline Coefficient & Add Back & Singles & Add Back Misty & Add Back Kerri \\
\hline$a_{0}$ & 0.156824 & 0.081394 & 0.071261 & 0.071432 \\
\hline$a_{1}$ & 0.63358 & 0.94916 & 0.68673 & 0.62892 \\
\hline$a_{2}$ & -0.001504 & 0.014860 & 0.003143 & -0.005765 \\
\hline$a_{3}$ & 2.20298 & 3.91351 & 1.26169 & 1.13341 \\
\hline$a_{4}$ & 26.3600 & 28.2898 & 28.2433 & 28.6916 \\
\hline
\end{tabular}

Table 39: Coefficients for efficiency equations for Type 1 source

\begin{tabular}{|c|c|c|c|c|}
\hline Coefficient & Add Back & Singles & Add Back Misty & Add Back Kerri \\
\hline$a_{0}$ & 0.149744 & 0.115100 & 0.082009 & 0.070029 \\
\hline$a_{1}$ & 0.66061 & 0.73177 & 0.62844 & 0.64205 \\
\hline$a_{2}$ & 0.012289 & -0.020786 & -0.004122 & -0.003608 \\
\hline$a_{3}$ & 2.38838 & 4.13703 & 1.24598 & 1.15564 \\
\hline$a_{4}$ & 25.9705 & 38.5222 & 29.3732 & 28.1108 \\
\hline
\end{tabular}




\section{Appendix 4: Fission Product Decay Schemes}

The following Figures represent the decay schemes for the fission products analyzed in the irradiated uranium samples for this research. They have been taken from the Table of Radionuclides[53,54]. 


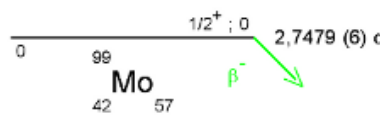

$\gamma$ Emission intensities per 100 disintegrations

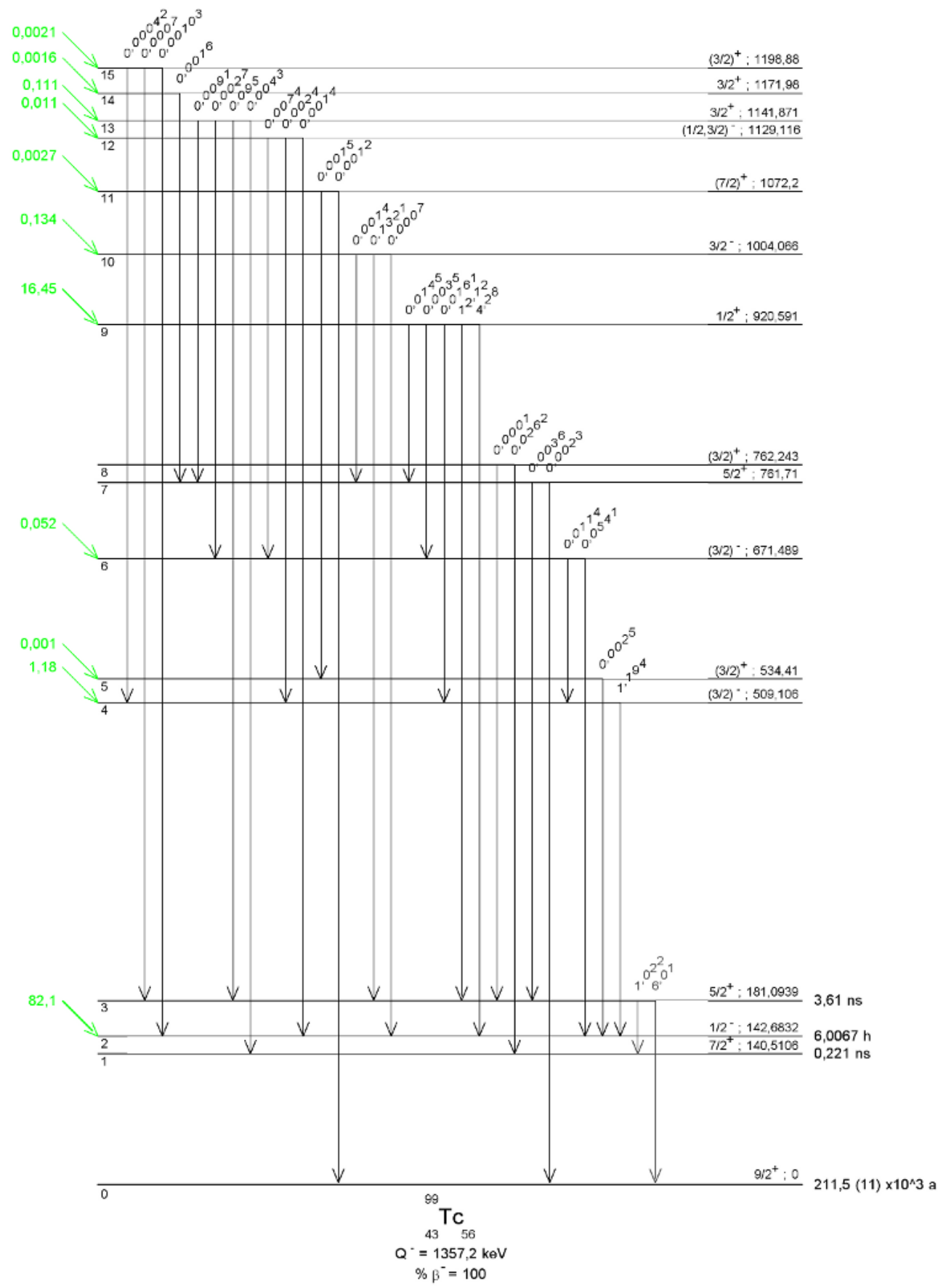

Figure 115: The decay scheme for ${ }^{99} \mathrm{Mo}$ 


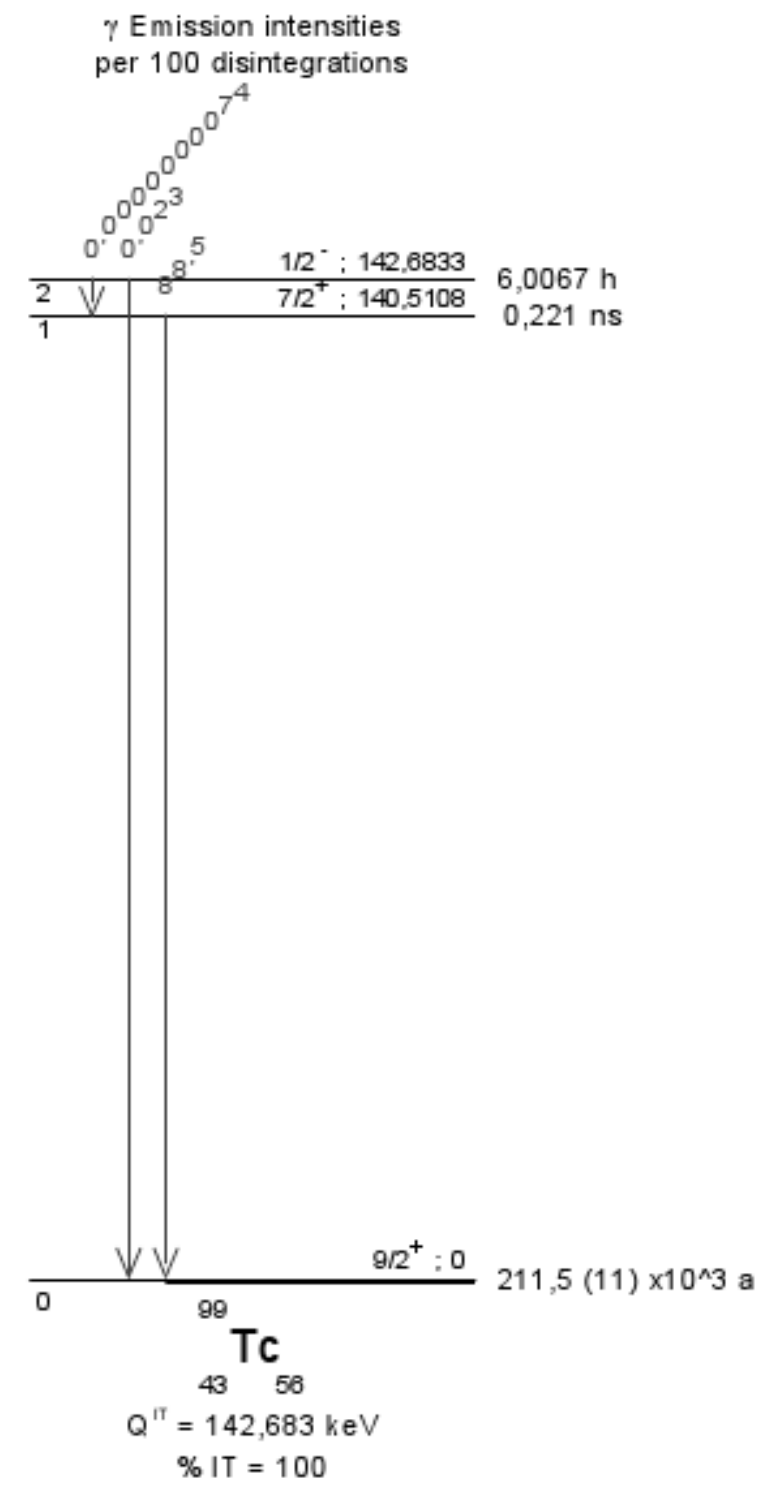

Figure 116: The decay scheme for ${ }^{99 m} \mathrm{Tc}$ 

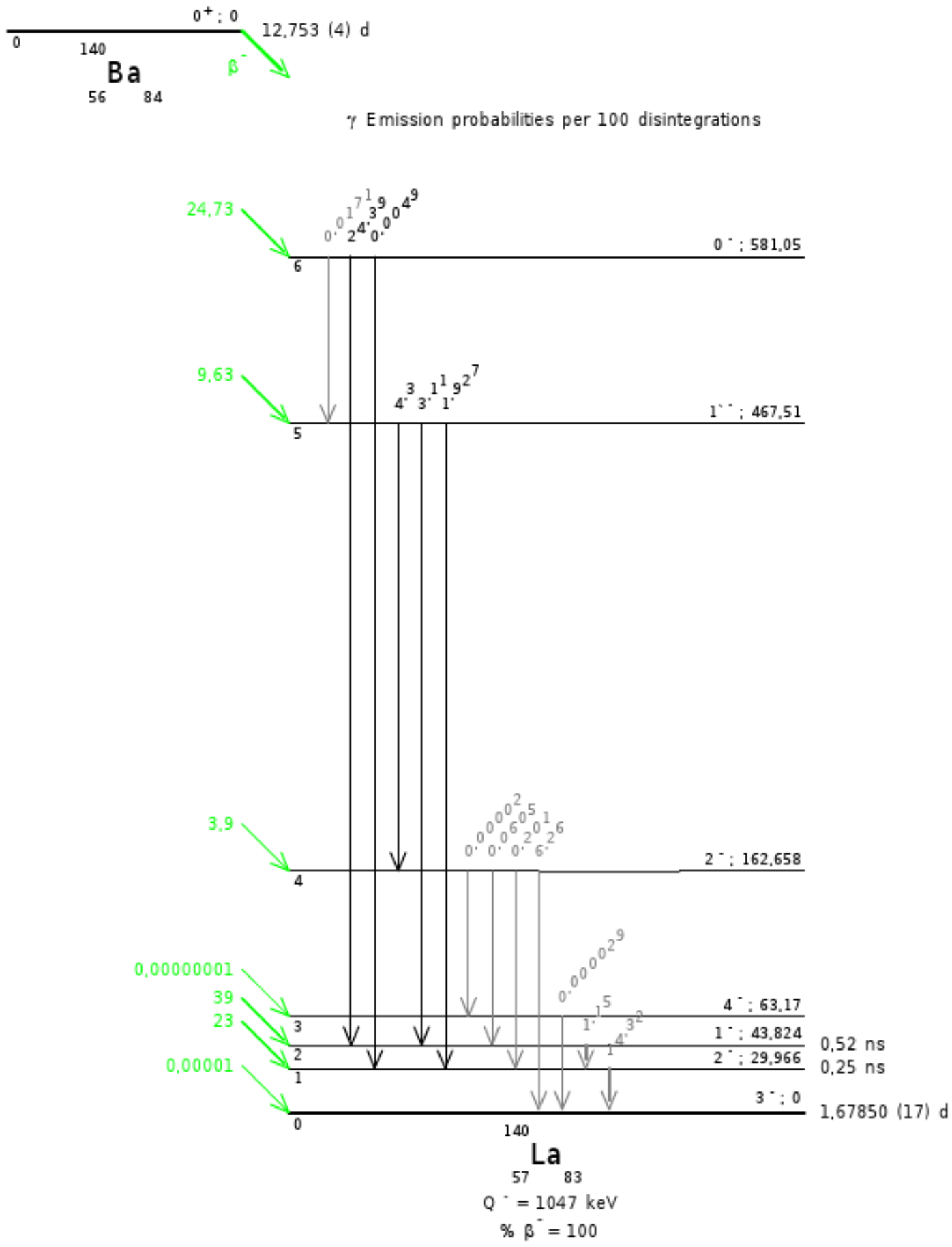

Figure 117: The decay scheme for ${ }^{140} \mathrm{Ba}$ 


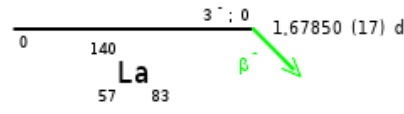

$\gamma$ Emission probabilities per 100 disintegrations

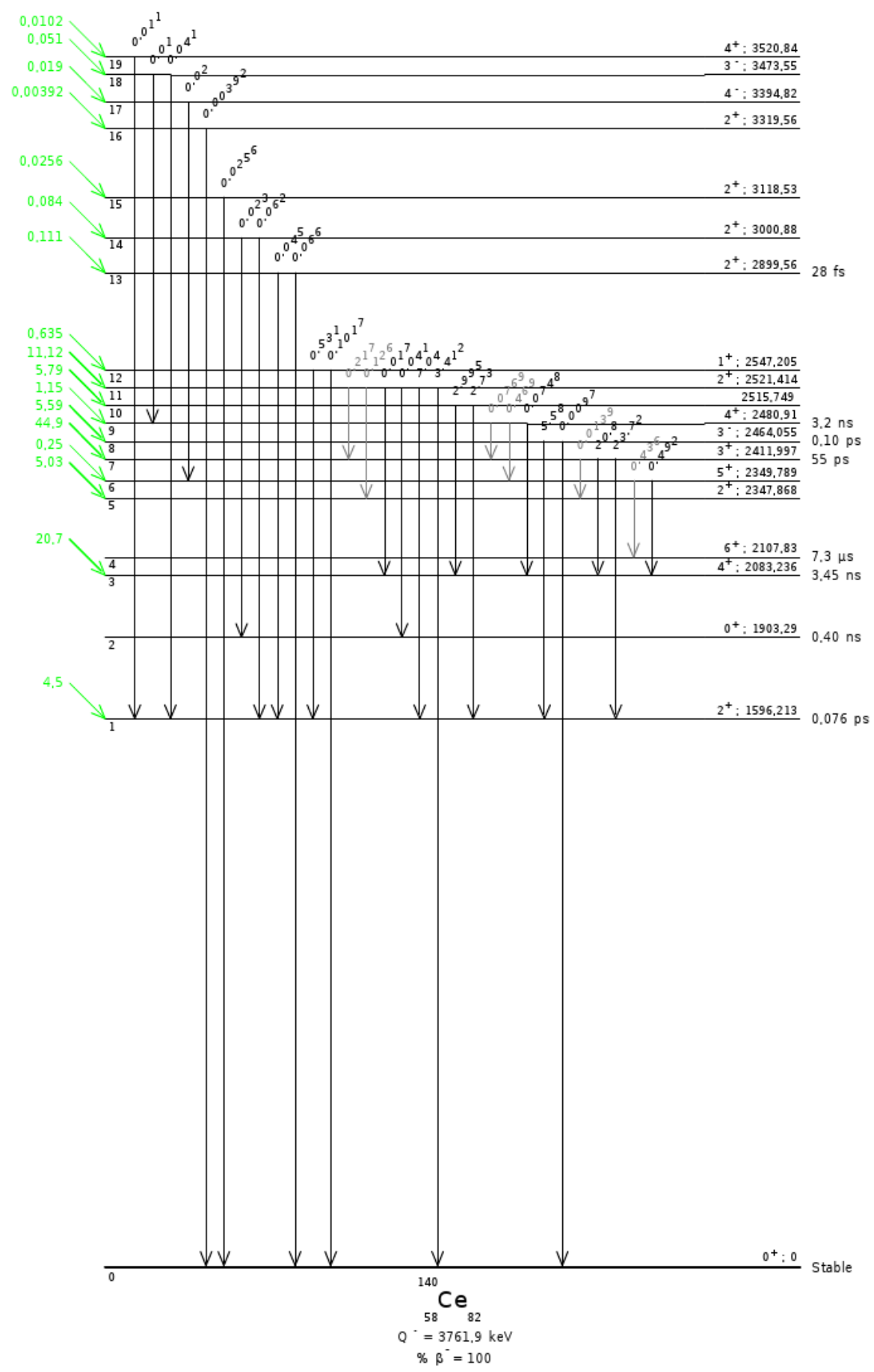

Figure 118: The decay scheme for ${ }^{140}$ La (Part 1) 


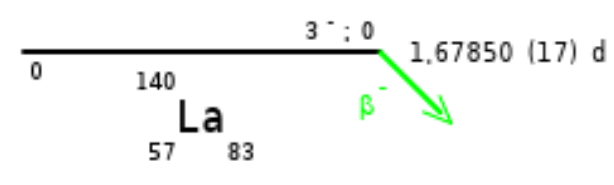
$\gamma$ Emission probabilities
per 100 disintegrations

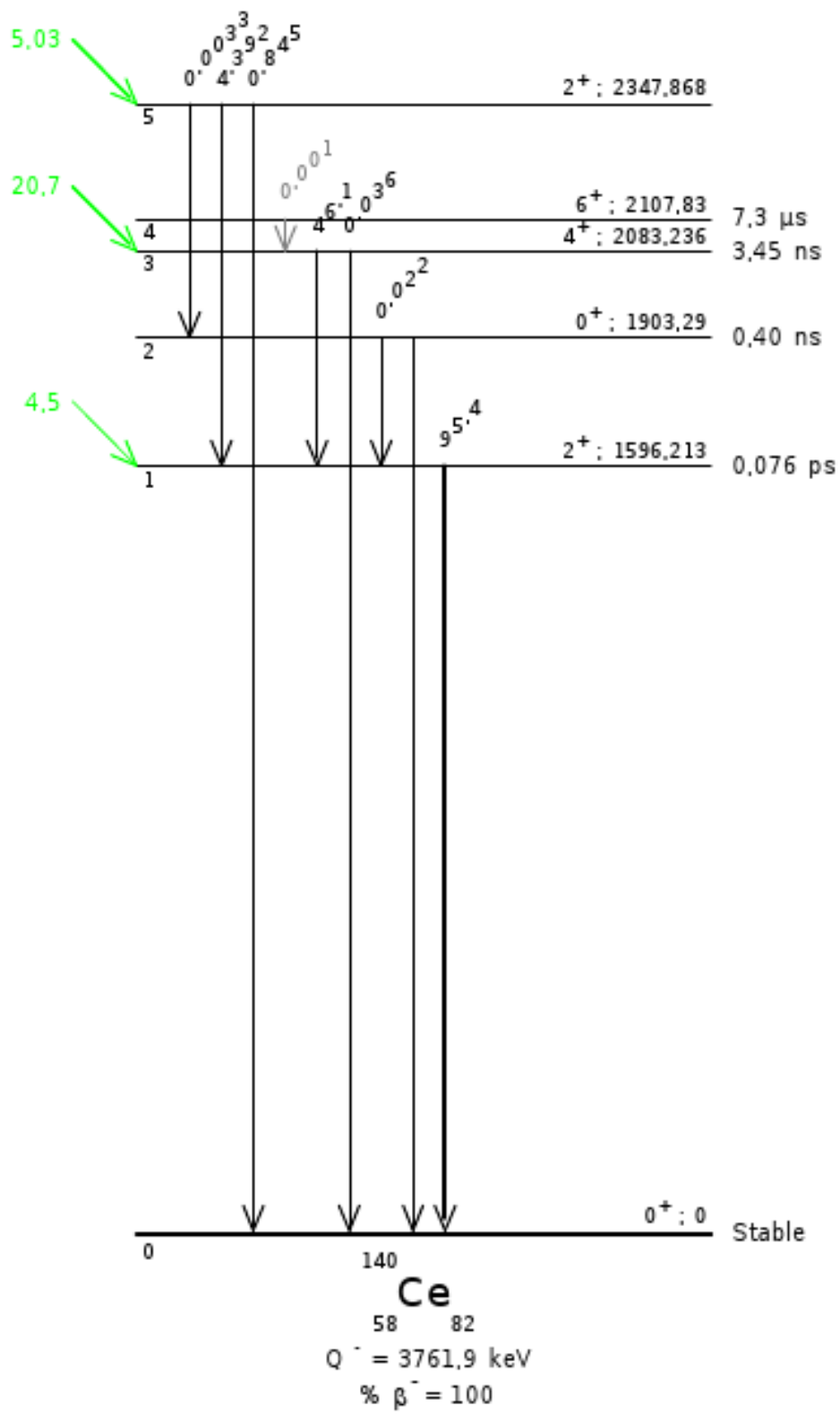

Figure 119: The decay scheme for ${ }^{140}$ La (Part 2) 


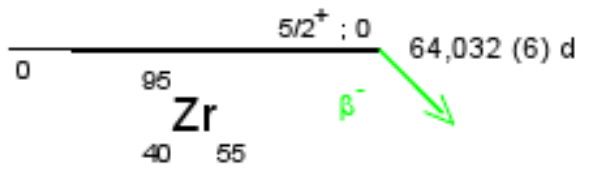

$\gamma$ Emission intensities per 100 disintegrations

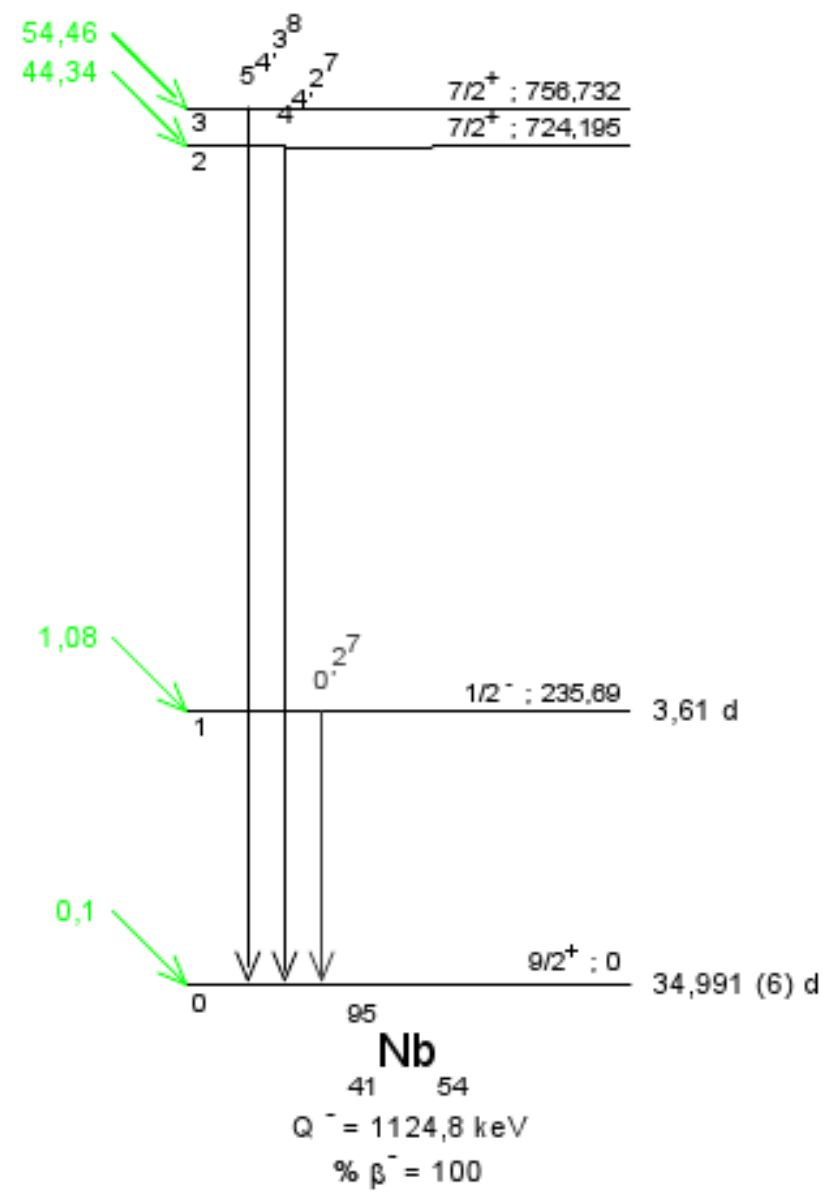

Figure 120: The decay scheme for ${ }^{95} \mathrm{Zr}$ 


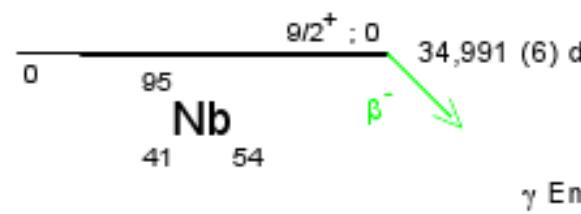

per 100 disintegrations

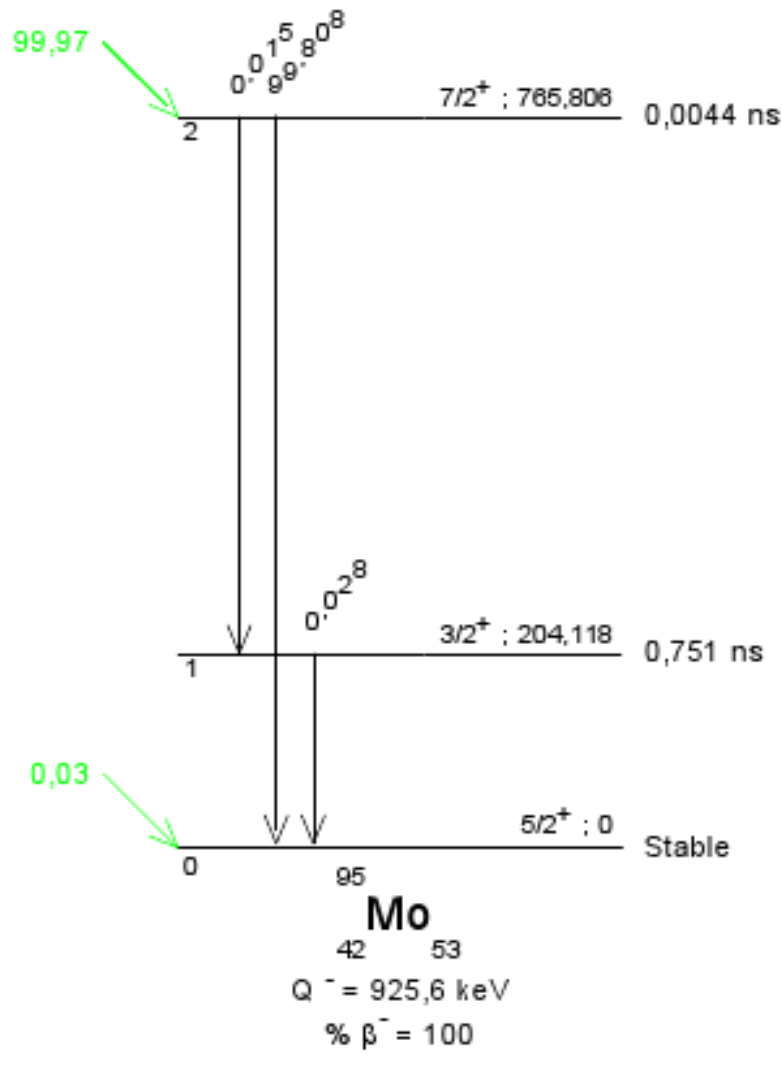

Figure 121: The decay scheme for ${ }^{95} \mathrm{Nb}$ 


\section{Appendix 5: Multi-Gamma Source Certifications}

\section{Eckert \& Ziegler}

\section{Analytics}

1380 Seaboard Industrial Blvd. Atlanta, Georgia 30318 Tel $404 \cdot 352 \cdot 8677$ Fax $404 \cdot 352 \cdot 2837$

www.analyticsinc.com

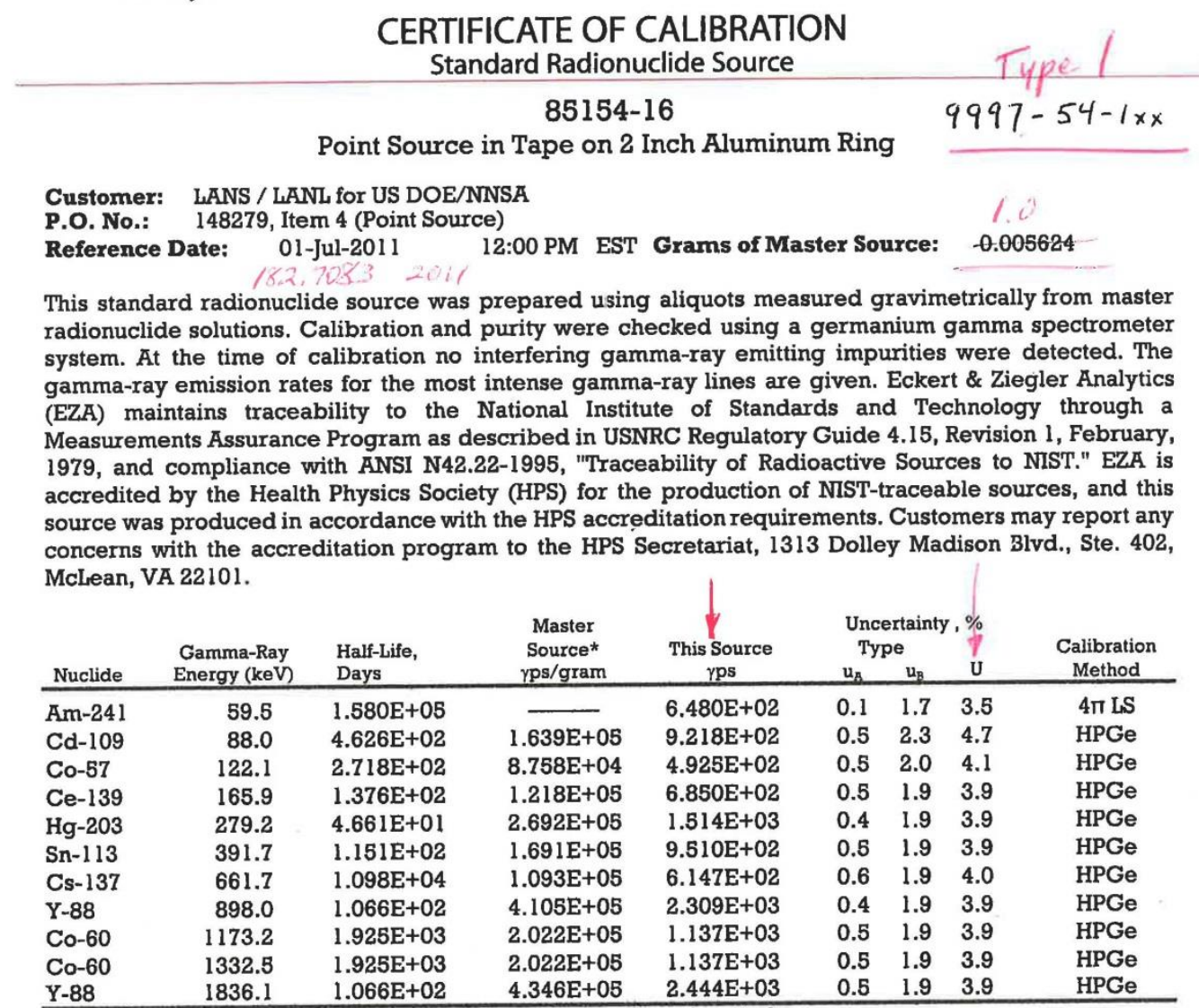

* Master Source refers to Analytics' 8-isotope mixture which is calibrated quarterly.

Calibration Methods: $4 \pi$ LS - 4 pi Liquid Scintillation Counting, HPGe - High Purity Germanium Gamma-Ray Spectrometer IC - Ionization Chamber. Uncertainty: U - Relative expanded uncertainty, k= 2. See NIST Technical Note 1297, "Guidelines for Evaluating and Expressing the Uncertainty of NIST Measurement Results."

(Certificate continued on reverse side)

\begin{tabular}{cc} 
MGS Certificate, Rev 2 09-28-2009 & Page 1 of 2 \\
\hline Corporate Office & Laboratory \\
24937 Avenue Tibbitts Valencia, California 91355 & 1380 Seaboard Industrial Blvd. Atlanta, Georgia, 30318
\end{tabular}

Figure 122: Type 1 Certificate 


\section{Eckert \& Ziegler}

1380 Seaboard Industrial Blvd.

Atlanta, Georgia 30318

Tel $404 \cdot 352 \cdot 8677$

Fax $404 \cdot 352 \cdot 2837$

Analytics

www.analyticsinc.com

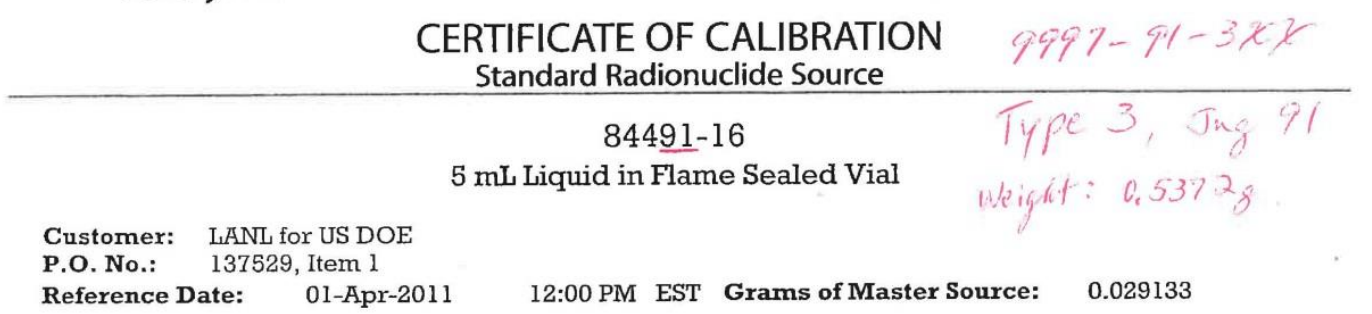

This standard radionuclide source was prepared using aliquots measured gravimetrically from master radionuclide solutions. Calibration and purity were checked using a germanium gamma spectrometer system. At the time of calibration no interfering gamma-ray emitting impurities were detected. The gamma ray emission rates for the most intense gamma-ray lines are given. Eckert \& Ziegler Analytics (EZA) maintains traceability to the National Institute of Standards and Technology through a Measurements Assurance Program as described in USNRC Regulatory Guide 4.15, Revision 1, February, 1979, and compliance with ANSI N42.22-1995, "Traceability of Radioactive Sources to NIST." EZA is accredited by the Health Physics Society (HPS) for the production of NIST-traceable sources, and this source was produced in accordance with the HPS accreditation requirements. Customers may report any concerns with the accreditation program to the HPS Secretariat, 1313 Dolley Madison Blvd., Ste. 402, McLean, VA 22101.

\begin{tabular}{|c|c|c|c|c|c|c|c|c|}
\hline Nuclide & $\begin{array}{c}\text { Gamma-Ray } \\
\text { Energy (keV) }\end{array}$ & $\begin{array}{l}\text { Half-Life, } \\
\text { Days }\end{array}$ & $\begin{array}{c}\text { Master } \\
\text { Source* } \\
\text { rps/gram }\end{array}$ & $\begin{array}{l}\text { This Source } \\
r \mathrm{ps} / 5.38315\end{array}$ & $\begin{array}{r}\text { Unc } \\
\text { Ty } \\
\mathrm{g}_{\mathrm{A}} \\
\end{array}$ & $\begin{array}{l}\text { rtaint } \\
\mathrm{u}_{\mathrm{B}} \\
\end{array}$ & $\begin{array}{r}\% \\
\mathrm{U} \\
\end{array}$ & $\begin{array}{c}\text { Calibration } \\
\text { Method }\end{array}$ \\
\hline Am-241 & 59.5 & $1.580 \mathrm{E}+05$ & $\longrightarrow$ & $3.327 \mathrm{E}+03$ & 0.1 & 0.9 & 1.8 & $4 \pi \mathrm{LS}$ \\
\hline Cd-109 & 88.0 & $4.626 \mathrm{E}+02$ & $1.659 \mathrm{E}+05$ & $4.833 \mathrm{E}+03$ & 0.6 & 1.7 & 3.6 & HPGe \\
\hline Co-57 & 122.1 & $2.718 \mathrm{E}+02$ & $8.949 E+04$ & $2.607 \mathrm{E}+03$ & 0.6 & 1.3 & 2.9 & HPGe \\
\hline Ce-139 & 165.9 & $1.376 \mathrm{E}+02$ & $1.247 \mathrm{E}+05$ & $3.633 E+03$ & 0.6 & 1.1 & 2.5 & HPGe \\
\hline $\mathrm{Hg}-203$ & 279.2 & $4.661 \mathrm{E}+01$ & 2.899E+05 & $8.446 \mathrm{E}+03$ & 0.6 & 1.1 & 2.5 & HPGe \\
\hline Sn-113 & 391.7 & $1.151 E+02$ & $1.739 \mathrm{E}+05$ & $5.066 \mathrm{E}+03$ & 0.6 & 1.1 & 2.5 & HPGe \\
\hline Cs-137 & 661.7 & $1.098 \mathrm{E}+04$ & $1.107 \mathrm{E}+05$ & $3.225 E+03$ & 0.8 & 1.2 & 2.9 & HPGe \\
\hline Y-88 & 898.0 & $1.066 \mathrm{E}+02$ & $4.246 E+05$ & $1.237 \mathrm{E}+04$ & 0.6 & 1.1 & 2.5 & HPGe \\
\hline Co-60 & 1173.2 & $1.925 \mathrm{E}+03$ & $2.118 \mathrm{E}+05$ & $6.170 \mathrm{E}+03$ & 0.7 & 1.1 & 2.6 & HPGe \\
\hline Co-60 & 1332.5 & $1.925 \mathrm{E}+03$ & $2.118 \mathrm{E}+05$ & $6.170 E+03$ & 0.7 & 1.1 & 2.6 & HPGe \\
\hline Y-88 & 1836.1 & $1.066 \mathrm{E}+02$ & 4.495E+05 & $1.310 \mathrm{E}+04$ & 0.7 & 1.1 & 2.6 & HPGe \\
\hline
\end{tabular}

* Master Source refers to Analytics' 8-isotope mixture which is calibrated quarterly.

Calibration Methods: 4r LS - 4 pi Liquid Scintillation Counting, HPGe - High Purity Germanium Gamma-Ray Spectrometer, IC Ionization Chamber. Uncertainty: U - Relative expanded uncertainty, $\mathrm{k}=2$. See NIST Technical Note 1297, "Guidelines for Evaluating and Expressing the Uncertainty of NIST Measurement Results."

(Certificate continued on reverse side)

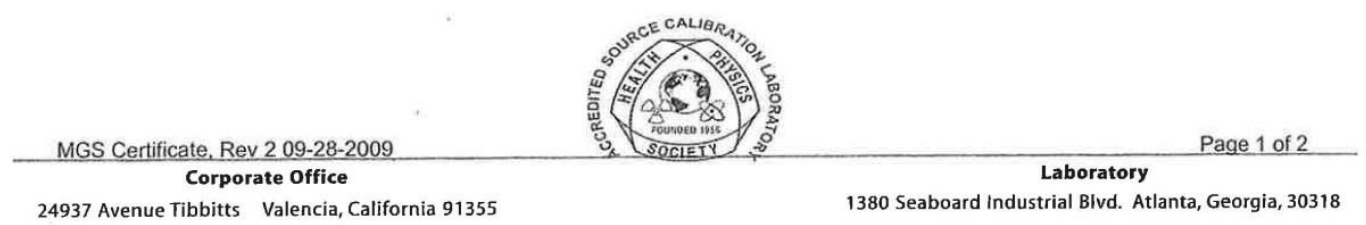

Figure 123: Type 3 Certificate (Page 1) 
Comments:

5.38315 grams $4 \mathrm{M} \mathrm{HCl}$ solution with approximately $30 \mu \mathrm{g} / \mathrm{g}$ each of $\mathrm{Cd}, \mathrm{Co}, \mathrm{Ce}, \mathrm{Hg}, \mathrm{Sn}, \mathrm{Cs}$, and Y carriers.

This standard will expire one year after the reference date.

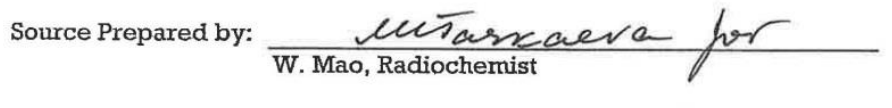

QA Approved:

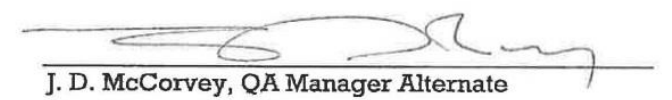

Date: $\quad 4 / 19 / 11$

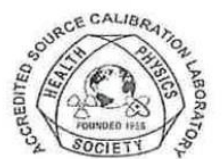

Page 2 of 2

Figure 124: Type 3 Certificate (Page 2) 


\section{References}

1 Hayes FN (1956) The International Journal of Applied Radiation and Isotopes 1:46-52

2 Eriksen VO, Jenssen G, Sunde A (1952) Physica 18:91-100

3 Tavendale AJ, Ewan GT (1963) Nuclear Instruments and Methods 25:185-187

4 Knoll G (2000) Radiation Detection and Measurement Hoboken:John Wiley \& Sons

5 Upp DL, Keyser RM, Twomey TR (2005) Journal of Radioanalytical and Nuclear Chemistry 264:121-126

6 Einstein A (1905) Annal der Physik 17:132

7 Fuggle JC, Martensson N (1980) Journal of Electron Spectroscopy and Related Phenomena 21:275-281

8 Compton A (1923) Physical Review 21:483-502

9 Evans RD (1982) The Atomic Nucleus Krieger, New York:

10 Crespi FCL, Camera F, Wieland O, Benzoni G, Brambilla S, Million B, Montanari D (2007) Nuclear Instruments and Methods A 570:459-466

11 Curie I, Joliot F (1933) Journal de Physique et le Radium 4:494-500

12 Duchene G, Beck FA, Twin PJ, de France G, Curien D, Han L, Beausang CW, Bentley MA, Nolan PJ, Simpson J (1999) Nuclear Instruments and Methods A 432:90-110

13 Debertin K, Helmer R (1988) Gamma- and x-ray spectrometry with semiconductor detectors

14 Dababneh S, Patronis N, Assimakopoulus PA, Gorres J, Heil M, Kappeler F, Karamanis D, O'Brien S, Reifarth R (2004) Nuclear Instruments and Methods in Physics Research A 517:230239

15 XIA L (2010) Pixie-4 Users Manual 2.10:

16 Nir-EI Y (2004) Applied Radiation and Isotopes 60:197-201

17 Deleplanque MA, Lee IY, Vetter K, Schmid GJ, Stephens FS, Clark RM, Diamond RM, Fallon P, Macchiavelli AO (1999) Nuclear Instruments and Methods in Physics Research A 430:292-310

18 Zhang W, Mekarski P, Dion M, Yi J, Ungar K (2012) Journal of Radioanalytical and Nuclear Chemistry 292:1265-1272 
19 Bing X, Weifan Y, Shuanggui Y, Taotao M, Jianjun H, Yanbing X, Zongwei L (1999) Journal of Radioanalytical and Nuclear Chemistry 242:163-165

20 Finn E, Metz L, Greenwood L, Pierson B, Friese J, Kephart R, Kephart J (2012) Journal of Radioanalytical and Nuclear Chemistry 293:267-272

21 Zhang W, Yi J, Mekarski P, Hoffman I, Ungar K, Leppanen AP (2011) J Radioanal Nucl Chem 287:551-555

22 Zhang W, Yi J, Mekarski P, Ungar K, Hauck B, Kramer GH (2011) Journal of Radioanalytical and Nuclear Chemistry 288:43-47

23 Burnett JL, Davies AV (2011) Journal of Radioanalytical and Nuclear Chemistry 288:699-703

24 Tomlin BE, Zeisler R, Lindstrom RM (2008) Nuclear Instruments and Methods A 589:243-249

25 Gill RD (1975) Gamma-Ray Angular Correlations Academic Press Inc., London:

26 Jenkins R, Gould RW, Gedcke D (1995) Quantitative X-ay Spectrometry Marcel Dekker, Inc., New York:

27 Brun R, Rademakers F (2007) ROOT User's Guide

28 Horne S, Jackman KR, Landsberger S (2012) Journal of Radioanalytical and Nuclear Chemistry 10.1007/s10967-012-2092-4:DOI

29 Garcia-Torano E, Pozuelo M, Salvat F (2005) Nuclear Instruments and Methods A 544:577583

30 Semkow TM, Mehmood G, Parekh PP, Virgil M (1990) Nuclear Instruments and Methods A 290:437-444

31 Singh B (2004) Nuclear Data Sheets 101:193-323

32 Basunia MS (2006) Nuclear Data Sheets 107:2323-2422

33 Holden NE, Hoffman DC (2000) Pure and Applied Chemistry 72:1525-1562

34 Blachot J (2006) Nuclear Data Sheets 107:355-506

35 Bhat MR (1992) Nuclear Data Sheets 67:195-270

36 Blachot J (2010) Nuclear Data Sheets 111:1471-1618

37 Sievers H (1991) Nuclear Data Sheets 62:271,273-325 
38 Muller HW (1987) Nuclear Data Sheets 50:1-62

39 Junde H, Su H (2006) Nuclear Data Sheets 107:1393-1530

40 Browne E, Tuli JK (2010) Nuclear Data Sheets 111:2425-2553

41 Gunnink R, De Corte F, De Wispelaere A, Bossus D, van Sluijs R, Vervecken D (1994) Lawrence Livermore National Laboratory Internal Report UCRL-JC-115368

42 Canberra Industries I (2002) Genie 2000 Spectroscopy System Operations Manual v2.1:

43 Kolotov VP, Atrashkevich VV, Gelsema SJ (1996) Journal of Radioanalytical and Nuclear Chemistry 210:183-196

44 Routti JT (1969) Lawrene Radiation Laboratory University of California Berkeley UCRL-19452:

45 Edgeworth FY (1908) Journal of the Royal Statistical Society 71:499-512

46 Schonfeld E (2004) http://www.nucleide.org/DDEP_WG/DDEPdata.htm

47 Jackman KR, Gritzo RE (2003) Los Alamos National Laboratory Internal Report LA-UR-0401691-34

48 Arnold D, Blaauw M, Fazinic S, Kolotov VP (2005) Nuclear Instruments and Methods A 536:196-210

49 Becquerel LNH (2012) Recommended Data http://www.nucleide.org/DDEP_WG/DDEPdata.htm:

50 Be MM, Chiste V, Dulieu C, Browne E, Chechev V, Kuzmenko N, Helmer R, Nichols A, Schonfeld E, Dersch R (2004) Table of Radionuclides (Vol. 1 - A = 1 to 150) Bureau International Des Poids Et Mesures:

51 Be MM, Duchemin B, Lame J, Morillon C, Piton F, Browne E, Chechev V, Helmer R, Schonfeld E (1999) Table of Radionuclides CEA-ISBN 2-7272-0200-8:

52 Nir-El Y (2012) Radiation Protection Dosimetry doi:10.1093/rpd/ncs106

53 Bevington PR (1969) Data Reduction and Error Analysis for the Physical Sciences New York:McGraw-Hill

54 Bateman H (1910) Proceedings of the Cambridge Philosophical Society 15:423-427

55 England TR, Rider BF (1994) Los Alamos National Laboratory LA-UR-94-3106: 
56 Wisniak J, Polishuk A (1999) Fluid Phase Equilibria 164:61-82

57 Cetnar J (2006) Annals of Nuclear Energy 33:640-645

58 Currie L (1968) Analytical Chemistry 40:

59 De Geer LE (2004) Applied Radiation and Isotopes 61:151-160

60 Chen J, Singh B, Cameron JA (2011) Nuclear Data Sheets 112:2357-3405

61 Bhattacharjee T, Chanda S, Battacharyya S, Basu SK, Bhowmik RK, Das JJ, Pramanik UD, Ghugre SS, Madhavan N, Mukherjee A, Mukherjee G, Muralithar S, Singh RP (2009) Nuclear Physics A 825:16-38

62 Sarkar MS, Kshetri R, Raut R, Mukherjee A, Sinha M, Ray M, Goswami A, Roy S, Basu P, Majumder H, Bhattacharya S, Dasmahapatra B (2006) Nuclear Instruments and Methods A $556: 266-272$

63 Duggan JL (2010) AN34 Overview http://www.ortec-online.com/Library/an34.aspx:Ortec Experiment 7 High-Resolution Gamma-Ray Spectroscopy 